Supporting Information for:

\title{
Reversal of Enantioselectivity in the Copper-Aminophenol Sulfonamide Catalyzed Alkynylation of Isatins by Slightly Tuning the Ligand structure and Basic Additives
}

Huakang He, Zinan Yang, Yu Chai, Ruoran Wu, Peng Chen, Jing Zhou*, and Hui Zhou* hzhou@cqmu.edu.cn; 102792@cqmu.edu.cn

Chongqing Research Center for Pharmaceutical Engineering, College of Pharmacy, Chongqing Medical University, Chongqing, China

\section{CONTENTS}

1. General information

2. General procedure for the synthesis of Ligands L1-L11

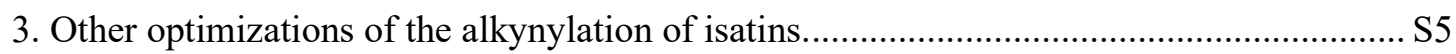

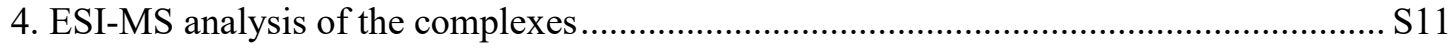

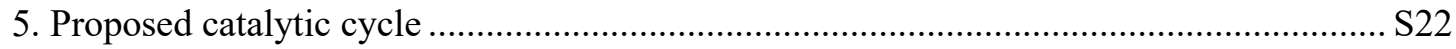

6. General procedure for the asymmetric alkynylation of isatins ...................................... S22

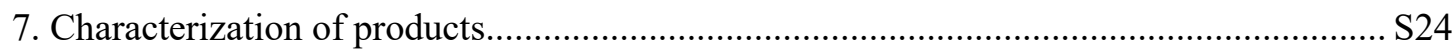

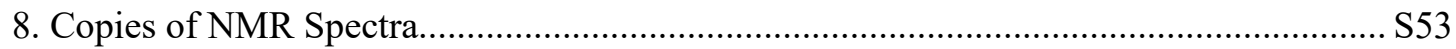




\section{General information}

All reagents were obtained from Acros, Adamas, or TCI etc. without further purification unless otherwise noted. High resolution mass spectra were recorded with Bruker UPLC-Q-TOF-MS (for the characterization of the compounds), Waters Acquity SQD (for the analysis of the complexes), or Bruker SolariX FT-ICR-MS (for the analysis of the complexes) system. NMR spectra were recorded on commercial instruments and operating at $600 \mathrm{MHz}$ for ${ }^{1} \mathrm{H} \mathrm{NMR}$ and $150 \mathrm{MHz}$ for ${ }^{13} \mathrm{C} \mathrm{NMR}$. Chemical shifts were reported in ppm from tetramethylsilane with the solvent resonance as the internal standard $\left(\mathrm{CDCl}_{3}, \delta=7.26\right.$ or $\left.\left(\mathrm{CD}_{3}\right)_{2} \mathrm{SO} \delta=2.50\right)$ in ${ }^{1} \mathrm{H}$ NMR spectra and Chemical shifts were reported in ppm from the tetramethylsilane with the solvent resonance as internal standard $\left(\mathrm{CDCl}_{3}, \delta=77.0\right.$ or $\left.\left(\mathrm{CD}_{3}\right)_{2} \mathrm{SO} \delta=39.5\right)$ in ${ }^{13} \mathrm{C} \mathrm{NMR}$ spectra. Spectra are reported as follows: chemical shift ( $\delta \mathrm{ppm})$, multiplicity ( $=$ singlet, $\mathrm{d}=$ doublet, $\mathrm{t}=$ triplet, $\mathrm{q}=$ quartet, $\mathrm{m}=$ multiplet $)$, coupling constants $(\mathrm{Hz})$, integration, and assignment. The enantiomeric excess (ee) was determined by HPLC analysis. Analytical HPLC was performed on a Shimadzu LC-16, using a chiral Daicel Chiralpak IA or ADH column at $254 \mathrm{~nm}$. Optical rotations were measured on a commercial polarimeter and are reported as follows: $[\alpha]_{\mathrm{D}}^{\mathrm{T}}(c=\mathrm{g} / 100 \mathrm{~mL}$, solvent $)$.

\section{General procedure for the synthesis of Ligands L1-L11}
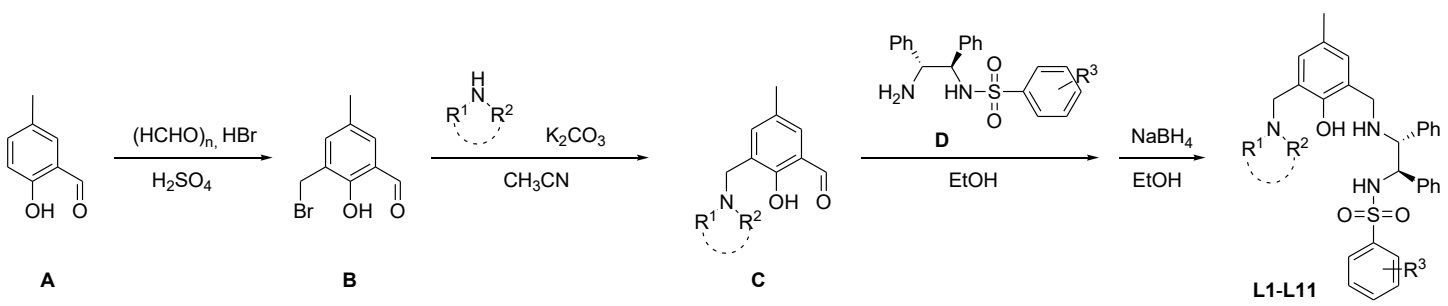

$\mathrm{HBr}(48 \% 6 \mathrm{~mL})$ was added into aldehyde $\mathbf{A}(10 \mathrm{mmol})$, followed by the additions of (HCHO)n (15 mmol) and several drops of concentrated sulfuric acid, the mixture was stirred at $70^{\circ} \mathrm{C}$ (oil bath) for $20 \mathrm{~h}$ under $\mathrm{N}_{2}$ atmosphere. Then the mixture was cooled to $\mathrm{rt}$ and extracted with $\mathrm{CH}_{2} \mathrm{Cl}_{2}$ and dried over $\mathrm{Na}_{2} \mathrm{SO}_{4}$. The solvent was removed in vacuo to give a white solid $(\mathbf{B}) ; \mathrm{K}_{2} \mathrm{CO}_{3}(6.25 \mathrm{mmol})$ was added into acetonitrile $(30 \mathrm{~mL})$, followed by the additions of the secondary amine $(6.25 \mathrm{mmol})$ and $\mathbf{B}(5.0 \mathrm{mmol})$, finally the mixture was stirred for $8 \mathrm{~h}$ at $85{ }^{\circ} \mathrm{C}$ (oil bath) under $\mathrm{N}_{2}$ atmosphere. The solvent was removed in vacuo and the mixture was extracted with $\mathrm{CH}_{2} \mathrm{Cl}_{2}$. The combined organic phase was washed with saturated sodium bicarbonate and dried over $\mathrm{Na}_{2} \mathrm{SO}_{4}$. The solvent was removed in vacuo to give a white solid (C). To a solution of $\mathbf{D}$ (3.6 mmol) in $50 \mathrm{~mL}$ ethanol was slowly added the aldehyde $\mathbf{C}$ (3.6 $\mathrm{mmol}$ ), and the mixture was stirred at $\mathrm{rt}$ until the reaction proceed completely detected by TLC. Sodium borohydride $(14.4 \mathrm{mmol})$ was then added in small portions at $0{ }^{\circ} \mathrm{C}$. 
And then the reaction was allowed to warm to ambient temperature and detected by TLC. It was poured over $120 \mathrm{~mL}$ of water and extracted with $\mathrm{CH}_{2} \mathrm{Cl}_{2}$. The combined organic phase was washed with Sat. $\mathrm{NaCl}$ and dried with $\mathrm{Na}_{2} \mathrm{SO}_{4}$, and the solvent was removed in vacuo and purified through column chromatography (petroleum ether /AcOEt $=3: 1)$ on silica gel to obtain the Ligand (L1-L11).

L1: White solid, $1.85 \mathrm{~g}, 88 \%$ yield (petroleum ether $/ \mathrm{AcOEt}=3: 1) ;{ }^{1} \mathrm{H} \mathrm{NMR}(600 \mathrm{MHz}$, $\left.\mathrm{CDCl}_{3}\right) \delta 7.36(\mathrm{~d}, J=8.1 \mathrm{~Hz}, 2 \mathrm{H}), 7.10-7.04(\mathrm{~m}, 6 \mathrm{H}), 7.00-6.97(\mathrm{~m}, 6 \mathrm{H}), 6.65(\mathrm{~d}, J=$ $11.0 \mathrm{~Hz}, 2 \mathrm{H}), 4.28(\mathrm{~d}, J=7.1 \mathrm{~Hz}, 1 \mathrm{H}), 3.72(\mathrm{~d}, J=7.1 \mathrm{~Hz}, 1 \mathrm{H}), 3.59$ (t, $J=14.7 \mathrm{~Hz}$, 2H), 3.50 (d, $J=13.9 \mathrm{~Hz}, 1 \mathrm{H}), 3.43$ (d, $J=13.1 \mathrm{~Hz}, 1 \mathrm{H}), 2.45$ (brs, 4H), 2.30 (s, 3H), $2.18(\mathrm{~s}, 3 \mathrm{H}), 1.69-1.37(\mathrm{~m}, 6 \mathrm{H}) .{ }^{13} \mathrm{C} \mathrm{NMR}\left(150 \mathrm{MHz}, \mathrm{CDCl}_{3}\right) \delta 153.9,142.3,139.5$, $138.9,137.1,129.0,128.9,128.9,128.3,128.1,127.9,127.7,127.4,127.4,127.2,127.1$, 127.0, 126.7, 126.5, 125.5, 120.9, 67.3, 63.1, 61.9, 53.7, 47.2, 25.7, 23.9, 21.3, 20.4. HRMS (ESI) $m / z$ : $[\mathrm{M}+\mathrm{H}]^{+}$Calcd for $\mathrm{C}_{35} \mathrm{H}_{42} \mathrm{~N}_{3} \mathrm{O}_{3} \mathrm{~S}^{+}$584.2941; Found: 584.2926 .

L2: White solid, $1.56 \mathrm{~g}, 74 \%$ yield (petroleum ether /AcOEt $=3: 1) ;{ }^{1} \mathrm{H}$ NMR $(600 \mathrm{MHz}$, $\left.\mathrm{CDCl}_{3}\right) \delta 7.34(\mathrm{~d}, J=8.1 \mathrm{~Hz}, 2 \mathrm{H}), 7.16-7.08(\mathrm{~m}, 3 \mathrm{H}), 7.06-7.02(\mathrm{~m}, 3 \mathrm{H}), 6.98-6.96(\mathrm{~m}$, $6 \mathrm{H}), 6.69(\mathrm{~s}, 2 \mathrm{H}), 4.29(\mathrm{~d}, J=7.3 \mathrm{~Hz}, 1 \mathrm{H}), 3.78-3.67(\mathrm{~m}, 5 \mathrm{H}), 3.65(\mathrm{~d}, J=13.7 \mathrm{~Hz}$, $1 \mathrm{H}), 3.61-3.53(\mathrm{~m}, 2 \mathrm{H}), 3.44$ (d, $J=13.1 \mathrm{~Hz}, 1 \mathrm{H}), 2.52$ (brs, 4H), 2.31 (s, 3H), 2.19 (s, $3 \mathrm{H}) .{ }^{13} \mathrm{C}$ NMR $\left(150 \mathrm{MHz}, \mathrm{CDCl}_{3}\right) \delta 153.3,142.4,139.4,138.7,137.1,129.4,128.9$, $128.3,128.2$, 127.8, 127.4, 127.2, 127.0, 125.6, 120.0, 67.3, 66.7, 63.1, 61.6, 52.8, 47.3, 21.3, 20.3. HRMS (ESI) $m / z$ : $[\mathrm{M}+\mathrm{H}]^{+}$Calcd for $\mathrm{C}_{34} \mathrm{H}_{40} \mathrm{~N}_{3} \mathrm{O}_{4} \mathrm{~S}^{+}$586.2734; Found: 586.2725 .

L3: White solid, $1.78 \mathrm{~g}, 87 \%$ yield (petroleum ether $/ \mathrm{AcOEt}=3: 1) ;{ }^{1} \mathrm{H} \mathrm{NMR}(600 \mathrm{MHz}$, $\left.\mathrm{CDCl}_{3}\right) \delta 7.36(\mathrm{~d}, J=8.2 \mathrm{~Hz}, 2 \mathrm{H}), 7.14-7.08(\mathrm{~m}, 3 \mathrm{H}), 7.05-7.02(\mathrm{~m}, 3 \mathrm{H}), 7.01-6.94(\mathrm{~m}$, $6 \mathrm{H}), 6.66(\mathrm{~d}, J=8.0 \mathrm{~Hz}, 2 \mathrm{H}), 4.27(\mathrm{~d}, J=7.3 \mathrm{~Hz}, 1 \mathrm{H}), 3.79-3.64(\mathrm{~m}, 3 \mathrm{H}), 3.60(\mathrm{~d}, J=$ $13.1 \mathrm{~Hz}, 1 \mathrm{H}), 3.41$ (d, $J=13.1 \mathrm{~Hz}, 1 \mathrm{H}), 2.58$ (brs, 4H), 2.30 (s, 3H), 2.18 (s, 3H), 1.82 (s, 4H). ${ }^{13} \mathrm{C} \mathrm{NMR}\left(150 \mathrm{MHz}, \mathrm{CDCl}_{3}\right) \delta 152.9,141.4,138.4,137.8,136.1,128.0,127.9$, 127.1, 126.5, 126.4, 126.4, 126.2, 126.1, 126.0, 124.4, 120.7, 66.3, 62.2, 57.6, 52.4, 46.3, 22.6, 20.3, 19.4. HRMS (ESI) m/z: $[\mathrm{M}+\mathrm{H}]^{+}$Calcd for $\mathrm{C}_{34} \mathrm{H}_{40} \mathrm{~N}_{3} \mathrm{O}_{3} \mathrm{~S}^{+}$570.2785; Found: 570.2793 .

L4: White solid, $1.99 \mathrm{~g}, 88 \%$ yield (petroleum ether $/ \mathrm{AcOEt}=3: 1) ;{ }^{1} \mathrm{H} \mathrm{NMR}(600 \mathrm{MHz}$, $\left.\mathrm{CDCl}_{3}\right) \delta 7.35(\mathrm{~d}, J=8.2 \mathrm{~Hz}, 2 \mathrm{H}), 7.13-7.09(\mathrm{~m}, 3 \mathrm{H}), 7.06-7.05(\mathrm{~m}, 3 \mathrm{H}), 7.03-6.95(\mathrm{~m}$, $6 \mathrm{H}), 6.66(\mathrm{~d}, J=17.4 \mathrm{~Hz}, 2 \mathrm{H}), 4.29(\mathrm{~d}, J=7.0 \mathrm{~Hz}, 1 \mathrm{H}), 3.74(\mathrm{~d}, J=7.0 \mathrm{~Hz}, 1 \mathrm{H}), 3.68$ $(\mathrm{d}, J=14.0 \mathrm{~Hz}, 1 \mathrm{H}), 3.61-3.55(\mathrm{~m}, 2 \mathrm{H}), 3.42(\mathrm{~d}, J=13.2 \mathrm{~Hz}, 1 \mathrm{H}), 2.5-2.42(\mathrm{~m}, 4 \mathrm{H})$, $2.30(\mathrm{~s}, 3 \mathrm{H}), 2.19$ (s, 3H), 1.50-1.46 (m, 4H), $1.30-1.24(\mathrm{~m}, 4 \mathrm{H}), 0.89$ (t, $J=7.4 \mathrm{~Hz}$, $6 \mathrm{H}) .{ }^{13} \mathrm{C} \mathrm{NMR}\left(150 \mathrm{MHz}, \mathrm{CDCl}_{3}\right) \delta 152.9,141.3,138.5,137.9,136.2,127.9,127.7$, $127.1,126.8,126.5,126.4,126.2,126.1,126.0,125.9,124.6,120.5,66.3,62.1,56.8$, 51.8, 46.1, 27.1, 20.3, 19.5, 19.4, 12.9. HRMS (ESI) $\mathrm{m} / \mathrm{z}:[\mathrm{M}+\mathrm{H}]^{+}$Calcd for 
$\mathrm{C}_{38} \mathrm{H}_{50} \mathrm{~N}_{3} \mathrm{O}_{3} \mathrm{~S}^{+}$628.3567; Found: 628.3577 .

L5: White solid, $2.25 \mathrm{~g}, 90 \%$ yield (petroleum ether $/ \mathrm{AcOEt}=3: 1) ;{ }^{1} \mathrm{H} \mathrm{NMR}(600 \mathrm{MHz}$, $\left.\mathrm{CDCl}_{3}\right) \delta 7.35-7.33(\mathrm{~m}, 6 \mathrm{H}), 7.30-7.24(\mathrm{~m}, 6 \mathrm{H}), 7.10-7.06(\mathrm{~m}, J=7.4,6.3 \mathrm{~Hz}, 3 \mathrm{H})$, 7.05-6.93 (m, 9H), $6.67(\mathrm{~d}, J=6.7 \mathrm{~Hz}, 2 \mathrm{H}), 4.30(\mathrm{~d}, J=6.9 \mathrm{~Hz}, 1 \mathrm{H}), 3.73(\mathrm{~d}, J=6.9$ $\mathrm{Hz}, 1 \mathrm{H}), 3.65$ (d, $J=13.9 \mathrm{~Hz}, 1 \mathrm{H}), 3.61-3.55$ (m, 4H), 3.54-3.49 (m, 3H), 2.30 (s, 3H), 2.18 (s, 3H). ${ }^{13} \mathrm{C}$ NMR $\left(150 \mathrm{MHz}, \mathrm{CDCl}_{3}\right) \delta 153.2,142.3,139.5,138.8,137.2,136.7$, $129.5,129.0,129.0,128.5,128.3,128.1,127.8,127.7,127.6,127.4,127.3,127.1,127.0$, 127.0, 125.6, 121.2, 67.2, 63.1, 57.6, 56.6, 47.0, 21.3, 20.4. HRMS (ESI) $m / z:[\mathrm{M}+\mathrm{H}]^{+}$ Calcd for $\mathrm{C}_{44} \mathrm{H}_{46} \mathrm{~N}_{3} \mathrm{O}_{3} \mathrm{~S}^{+}$696.3254; Found: 696.3242 .

L6: Pale yellow solid, $1.95 \mathrm{~g}, 88 \%$ yield (petroleum ether $/ \mathrm{AcOEt}=3: 1) ;{ }^{1} \mathrm{H}$ NMR $(600$ $\left.\mathrm{MHz}, \mathrm{CDCl}_{3}\right) \delta 7.96(\mathrm{~d}, J=8.3 \mathrm{~Hz}, 2 \mathrm{H}), 7.56(\mathrm{~d}, J=8.3 \mathrm{~Hz}, 2 \mathrm{H}), 7.18-6.90(\mathrm{~m}, 10 \mathrm{H})$, $6.64(\mathrm{~d}, J=19.9 \mathrm{~Hz}, 2 \mathrm{H}), 4.41(\mathrm{~d}, J=6.6 \mathrm{~Hz}, 1 \mathrm{H}), 3.77(\mathrm{~d}, J=6.2 \mathrm{~Hz}, 1 \mathrm{H}), 3.61(\mathrm{t}, J$ $=14.4 \mathrm{~Hz}, 2 \mathrm{H}), 3.55-3.53(\mathrm{~m}, 1 \mathrm{H}), 3.44(\mathrm{~d}, J=12.9 \mathrm{~Hz}, 1 \mathrm{H}), 2.48$ (brs, 4H), 2.17 (s, $3 \mathrm{H}), 1.62-1.49(\mathrm{~m}, 6 \mathrm{H}) .{ }^{13} \mathrm{C} \mathrm{NMR}\left(150 \mathrm{MHz}, \mathrm{CDCl}_{3}\right) \delta 154.0,149.2,146.2,139.2$, 138.2, 129.1, 128.3, 128.1, 128.0, 128.0, 127.4, 127.4, 127.4, 127.3, 125.3, 123.4, 66.9, 63.5, 61.8, 53.8, 47.6, 25.7, 23.9, 20.3. HRMS (ESI) $m / z:[\mathrm{M}+\mathrm{H}]^{+}$Calcd for $\mathrm{C}_{34} \mathrm{H}_{39} \mathrm{~N}_{4} \mathrm{O}_{5} \mathrm{~S}^{+}$615.2636; Found: 615.2626.

L7: Pale yellow solid, $1.92 \mathrm{~g}, 87 \%$ yield (petroleum ether $/ \mathrm{AcOEt}=3: 1) ;{ }^{1} \mathrm{H}$ NMR $(600$ $\left.\mathrm{MHz} \mathrm{CDCl}_{3}\right) \delta 8.15-8.14(\mathrm{~m}, 2 \mathrm{H}), 7.75(\mathrm{~d}, J=7.7 \mathrm{~Hz}, 1 \mathrm{H}), 7.36(\mathrm{t}, J=8.3 \mathrm{~Hz}, 1 \mathrm{H})$, 7.11-7.07 (m, 3H), 7.03-7.00 (m, 7H), $6.66(\mathrm{~d}, J=5.6 \mathrm{~Hz}, 2 \mathrm{H}), 4.44(\mathrm{~d}, J=7.2 \mathrm{~Hz}, 1 \mathrm{H})$, $3.76(\mathrm{~d}, J=7.1 \mathrm{~Hz}, 1 \mathrm{H}), 3.62(\mathrm{t}, J=11.9 \mathrm{~Hz}, 2 \mathrm{H}), 3.55-3.53(\mathrm{~m}, 1 \mathrm{H}), 3.45(\mathrm{~d}, J=12.9$ $\mathrm{Hz}, 1 \mathrm{H}), 2.48$ (brs, 4H), 2.18 (s, 3H), 1.69-1.40 (m, 6H). $\left.{ }^{13} \mathrm{C} \mathrm{NMR} \mathrm{(150} \mathrm{MHz,} \mathrm{CDCl}_{3}\right)$ $\delta 154.0,147.6,142.6,139.2,137.9,132.4,129.4,129.1,128.2,128.1,128.0,127.5$, 127.4, 127.3, 126.1, 125.3, 122.3, 121.0, 66.9, 63.5, 61.9, 53.8, 47.6, 25.7, 23.9, 20.3. HRMS (ESI) $m / z:[\mathrm{M}+\mathrm{H}]^{+}$Calcd for $\mathrm{C}_{34} \mathrm{H}_{39} \mathrm{~N}_{4} \mathrm{O}_{5} \mathrm{~S}^{+}$615.2636; Found: 615.2630.

L8: Pale yellow solid, $1.79 \mathrm{~g}, 81 \%$ yield (petroleum ether $/ \mathrm{AcOEt}=3: 1) ;{ }^{1} \mathrm{H}$ NMR $(600$ $\left.\mathrm{MHz}, \mathrm{CDCl}_{3}\right) \delta 7.64(\mathrm{~d}, J=7.4 \mathrm{~Hz}, 1 \mathrm{H}), 7.48-7.39$ (m, 2H), 7.27-7.24 (m, 1H), 7.14$7.13(\mathrm{~m}, 2 \mathrm{H}), 7.09-7.00(\mathrm{~m}, 8 \mathrm{H}), 6.63(\mathrm{~d}, J=10.4 \mathrm{~Hz}, 2 \mathrm{H}), 4.65(\mathrm{~d}, J=6.0 \mathrm{~Hz}, 1 \mathrm{H})$, $3.91(\mathrm{~d}, J=6.0 \mathrm{~Hz}, 1 \mathrm{H}), 3.65(\mathrm{~d}, J=13.7 \mathrm{~Hz}, 2 \mathrm{H}), 3.46(\mathrm{dd}, J=34.2,13.7 \mathrm{~Hz}, 2 \mathrm{H})$, 2.46 (brs, 4H), 2.17 (s, 3H), 1.66-1.36 (m, 6H). ${ }^{13} \mathrm{C} \mathrm{NMR}\left(150 \mathrm{MHz}, \mathrm{CDCl}_{3}\right) \delta 153.9$, $147.2,139.4,138.7,134.4,132.4,132.2,130.2,129.0,127.9,127.9,127.7,127.3,127.3$, $127.2,127.1,125.5,124.6,120.9,66.4,64.5,61.9,53.8,47.3,25.7,24.0,20.4$. HRMS (ESI) $m / z:[\mathrm{M}+\mathrm{H}]^{+}$Calcd for $\mathrm{C}_{34} \mathrm{H}_{39} \mathrm{~N}_{4} \mathrm{O}_{5} \mathrm{~S}^{+}$615.2636; Found: 615.2627.

L9: White solid, $1.93 \mathrm{~g}, 90 \%$ yield (petroleum ether $/ \mathrm{AcOEt}=3: 1) ;{ }^{1} \mathrm{H} \mathrm{NMR}(600 \mathrm{MHz}$, $\left.\mathrm{CDCl}_{3}\right) \delta 7.39(\mathrm{~d}, J=8.8 \mathrm{~Hz}, 2 \mathrm{H}), 7.15-7.09(\mathrm{~m}, 4 \mathrm{H}), 7.07-6.98(\mathrm{~m}, 6 \mathrm{H}), 6.69-6.63(\mathrm{~m}$, $4 \mathrm{H}), 4.27(\mathrm{~d}, J=7.2 \mathrm{~Hz}, 1 \mathrm{H}), 3.77(\mathrm{~s}, 3 \mathrm{H}), 3.71(\mathrm{~d}, J=7.2 \mathrm{~Hz}, 1 \mathrm{H}), 3.64-3.56(\mathrm{~m}, 2 \mathrm{H})$, 3.51 (d, $J=13.9 \mathrm{~Hz}, 1 \mathrm{H}), 3.43$ (d, $J=13.1 \mathrm{~Hz}, 1 \mathrm{H}), 2.45$ (brs, 4H), 2.18 (s, 3H), 1.70- 
$1.38(\mathrm{~m}, 6 \mathrm{H}) .{ }^{13} \mathrm{C} \mathrm{NMR}\left(150 \mathrm{MHz}, \mathrm{CDCl}_{3}\right) \delta 162.1,153.9,139.5,138.8,131.9,129.1$, $128.9,128.9,128.3,128.1,127.9,127.8,127.5,127.2,127.2,127.0,127.0,126.5,125.6$, $113.5,67.4,63.2,55.4,53.8,47.3,25.7,23.9,20.4$. HRMS (ESI) $m / z:[\mathrm{M}+\mathrm{H}]^{+} \mathrm{Calcd}$ for $\mathrm{C}_{35} \mathrm{H}_{42} \mathrm{~N}_{3} \mathrm{O}_{4} \mathrm{~S}^{+} 600.2891$; Found: 600.2881 .

L10: White solid, $2.01 \mathrm{~g}, 91 \%$ yield (petroleum ether $/ \mathrm{AcOEt}=3: 1) ;{ }^{1} \mathrm{H}$ NMR $(600$ $\left.\mathrm{MHz}, \mathrm{CDCl}_{3}\right) \delta$ 7.10-7.06 (m, 3H), 6.97-6.89 (m, 3H), $6.86(\mathrm{t}, J=7.5 \mathrm{~Hz}, 2 \mathrm{H}), 6.78-$ $6.77(\mathrm{~m}, 3 \mathrm{H}), 6.66-6.66(\mathrm{~m}, 3 \mathrm{H}), 4.26(\mathrm{~d}, J=8.8 \mathrm{~Hz}, 1 \mathrm{H}), 3.67-3.58(\mathrm{~m}, 3 \mathrm{H}), 3.52(\mathrm{t}, J$ $=12.3 \mathrm{~Hz}, 2 \mathrm{H}), 2.60-2.43(\mathrm{~m}, 4 \mathrm{H}), 2.37(\mathrm{~s}, 6 \mathrm{H}), 2.20(\mathrm{~s}, 3 \mathrm{H}), 2.17(\mathrm{~s}, 3 \mathrm{H}), 1.66-1.38$ $(\mathrm{m}, 6 \mathrm{H}) .{ }^{13} \mathrm{C} \mathrm{NMR}\left(150 \mathrm{MHz}, \mathrm{CDCl}_{3}\right) \delta 154.0,141.3,139.5,138.8,138.1,134.3,131.3$, 129.0, 128.0, 127.9, 127.5, 127.4, 127.3, 127.2, 127.1, 126.8, 125.6, 120.9, 67.8, 63.5, $62.0,53.8,47.3,31.5,25.7,23.9,22.7,20.7,20.4,14.1$. HRMS (ESI) $m / z:[\mathrm{M}+\mathrm{H}]^{+}$ Calcd for $\mathrm{C}_{37} \mathrm{H}_{46} \mathrm{~N}_{3} \mathrm{O}_{3} \mathrm{~S}^{+}$612.3254; Found: 612.3257.

L11: White solid, $1.98 \mathrm{~g}, 89 \%$ yield (petroleum ether /AcOEt = 3:1); ${ }^{1} \mathrm{H}$ NMR $(600$ $\left.\mathrm{MHz}, \mathrm{CDCl}_{3}\right) \delta$ 7.96-7.95 (m, 1H), $7.79(\mathrm{~d}, J=8.1 \mathrm{~Hz}, 1 \mathrm{H}), 7.72-7.64(\mathrm{~m}, 2 \mathrm{H}), 7.56$ $(\mathrm{t}, J=7.4 \mathrm{~Hz}, 1 \mathrm{H}), 7.52-7.49(\mathrm{~m}, 2 \mathrm{H}), 7.01-6.94(\mathrm{~m}, 10 \mathrm{H}), 6.66(\mathrm{~d}, J=5.1 \mathrm{~Hz}, 2 \mathrm{H})$, $4.36(\mathrm{~d}, J=7.2 \mathrm{~Hz}, 1 \mathrm{H}), 3.73(\mathrm{~d}, J=7.2 \mathrm{~Hz}, 1 \mathrm{H}), 3.59$ (dd, $J=18.4,13.5 \mathrm{~Hz}, 2 \mathrm{H}), 3.52$ $(\mathrm{d}, J=13.9 \mathrm{~Hz}, 1 \mathrm{H}), 3.44$ (d, $J=13.1 \mathrm{~Hz}, 1 \mathrm{H}), 2.46$ (brs, 4H), 2.18 (s, 3H), 1.60-1.47 (m, 6H). ${ }^{13} \mathrm{C} \mathrm{NMR}\left(150 \mathrm{MHz}, \mathrm{CDCl}_{3}\right) \delta 153.9,139.4,138.6,137.1,134.4,131.8,129.1$, $128.9,128.8,128.6,128.3,128.2,128.2,128.1,128.0,127.9,127.7,127.5,127.4,127.3$, 127.3, 127.2, 127.1, 126.9, 126.8, 126.3, 125.6, 122.4, 67.3, 63.3, 53.8, 47.4, 25.7, 23.9, 20.4. HRMS (ESI) $\mathrm{m} / z$ : $[\mathrm{M}+\mathrm{H}]^{+}$Calcd for $\mathrm{C}_{38} \mathrm{H}_{42} \mathrm{~N}_{3} \mathrm{O}_{3} \mathrm{~S}^{+}$620.2941; Found: 620.2936.

\section{Other optimizations of the alkynylation of isatins.}

Table S1: The effects of the different metal salt. ${ }^{a}$

\begin{tabular}{|c|c|c|c|c|}
\hline Entry & $\begin{array}{c}\text { Ligand } \\
(10 \mathrm{~mol} \%)\end{array}$ & Metal salt (20 mol\%) & Yield $(\%)^{b}$ & $e e(\%)^{c}$ \\
\hline 1 & L1 & CuOTf $\cdot 0.5 \mathrm{PhH}$ & 54 & $76(R)$ \\
\hline 2 & $\mathbf{L 1}$ & $\mathrm{Cu}(\mathrm{OTf})_{2}$ & 52 & $61(S)$ \\
\hline 3 & $\mathbf{L 1}$ & $\mathrm{CuOAc}$ & trace & \\
\hline 4 & $\mathbf{L 1}$ & $\mathrm{CuI}$ & trace & \\
\hline 5 & L1 & $\mathrm{Cu}(\mathrm{OAc})_{2} \cdot \mathrm{H}_{2} \mathrm{O}$ & trace & \\
\hline 6 & L1 & $\mathrm{Cu}\left(\mathrm{ClO}_{4}\right)_{2} \cdot 6 \mathrm{H}_{2} \mathrm{O}$ & 30 & $59(R)$ \\
\hline
\end{tabular}

Table S2: The effects of the molar ratio of $\mathbf{L 1} / \mathrm{CuOTf} \cdot 0.5 \mathrm{PhH} .{ }^{a}$

\begin{tabular}{llcll}
\hline Entry & The amount of $\mathbf{L 1}$ & $\begin{array}{c}\text { The amount of } \\
\text { CuOTf } 0.5 \mathrm{PhH}\end{array}$ & Yield $(\%)^{b}$ & ee $(\%)^{c}$ \\
\hline
\end{tabular}




\begin{tabular}{lcccc}
\hline 1 & $10 \mathrm{~mol} \%$ & $10 \mathrm{~mol} \%$ & trace & \\
2 & $10 \mathrm{~mol} \%$ & $20 \mathrm{~mol} \%$ & 54 & $76(R)$ \\
3 & $10 \mathrm{~mol} \%$ & $30 \mathrm{~mol} \%$ & 97 & $77(R)$ \\
4 & $10 \mathrm{~mol} \%$ & $40 \mathrm{~mol} \%$ & 90 & $76(S)$ \\
5 & $10 \mathrm{~mol} \%$ & $5 \mathrm{~mol} \%$ & trace & \\
6 & $20 \mathrm{~mol} \%$ & $10 \mathrm{~mol} \%$ & trace &
\end{tabular}

${ }^{a}$ Unless otherwise noted, all reactions were performed with $1 \mathrm{a}(0.1 \mathrm{mmol}), \mathbf{2 a}(0.4 \mathrm{mmol})$, TFE $(1.0 \mathrm{~mL}), \mathrm{Et}_{3} \mathrm{~N}(20 \mathrm{~mol} \%)$, at $\mathrm{rt}$ for $24 \mathrm{~h} .{ }^{b}$ Isolated yield. ${ }^{c}$ Determined by HPLC.

Table S3: The effects of the Solvent. ${ }^{a}$

\begin{tabular}{|c|c|c|c|}
\hline Entry & Solvent & Yield $(\%)^{b}$ & $e e(\%)^{c}$ \\
\hline 1 & $\mathrm{CH}_{2} \mathrm{Cl}_{2}$ & 42 & $63(R)$ \\
\hline 2 & $\mathrm{AcOEt}$ & trace & \\
\hline 3 & THF & trace & \\
\hline 4 & $\mathrm{CH}_{3} \mathrm{CN}$ & trace & \\
\hline 5 & $\mathrm{CH}_{3} \mathrm{OH}$ & 63 & $49(R)$ \\
\hline 6 & $\mathrm{EtOH}$ & 65 & $65(R)$ \\
\hline 7 & IPA & 72 & $74(R)$ \\
\hline 8 & TFE & 97 & $77(R)$ \\
\hline 9 & HFIP & 43 & $60(R)$ \\
\hline 10 & Toluene & 38 & $48(S)$ \\
\hline \multicolumn{4}{|c|}{$\begin{array}{l}{ }^{a} \text { Unless otherwise noted, all reactions were performed with } \mathbf{L 1} / \mathrm{CuOTf} \cdot 0.5 \mathrm{PhH}(1 / 3,10 \\
\text { mol\%), 1a }(0.1 \mathrm{mmol}), \mathbf{2 a}(0.4 \mathrm{mmol}), \mathrm{TFE}(1.0 \mathrm{~mL}), \mathrm{Et}_{3} \mathrm{~N}(20 \mathrm{~mol} \%) \text {, at } \mathrm{rt} \text { for } 24 \mathrm{~h}^{b} \text { Isolated } \\
\text { yield. }{ }^{c} \text { Determined by HPLC. }\end{array}$} \\
\hline
\end{tabular}

Table S4: The effects of different bases (20 mol\%) for copper / L1-mediated alkynylation of isatins.. ${ }^{a}$

\begin{tabular}{llll}
\hline Entry & Base & ${\text { Yield }(\%)^{b}}^{b}$ & $e e(\%)^{c}$ \\
\hline 1 & Et $_{3} \mathrm{~N}(20 \mathrm{~mol} \%)$ & 97 & $77(R)$ \\
2 & $i-$ Pr $_{2} \mathrm{NEt}(20 \mathrm{~mol} \%)$ & 54 & $78(R)$ \\
3 & $\mathrm{Cy}_{2} \mathrm{NMe}^{d}(20 \mathrm{~mol} \%)$ & 50 & $65(R)$ \\
4 & $\mathrm{DBU}^{e}(20 \mathrm{~mol} \%)$ & 82 & $64(R)$ \\
5 & $\operatorname{DMAP}^{f}(20 \mathrm{~mol} \%)$ & 98 & $73(S)$ \\
$6^{g}$ & $\operatorname{Pyridine~}(20 \mathrm{~mol} \%)_{7}$ & 87 & $45(S)$ \\
7 & $n-\mathrm{Bu}_{2} \mathrm{NH}(20 \mathrm{~mol} \%)$ & 63 & $73(R)$ \\
8 & $\mathrm{Cy}_{2} \mathrm{NH}^{h}(20 \mathrm{~mol} \%)$ & 95 & $72(S)$ \\
9 & $i-\mathrm{Pr}_{2} \mathrm{NH}^{2}(20 \mathrm{~mol} \%)$ & 98 & $42(R)$ \\
10 & $\mathrm{TMP}^{i}(20 \mathrm{~mol} \%)$ & 80 & $69(R)$
\end{tabular}


${ }^{a}$ Unless otherwise noted, all reactions were carried out with L1 (10 mol\%), CuOTf $\cdot 0.5 \mathrm{PhH}(30$ mol\%), $\mathbf{1 a}(0.1 \mathrm{mmol}), \mathbf{2 a}(0.4 \mathrm{mmol})$ and base, in trifluoroethanol $(1.0 \mathrm{~mL})$ at $\mathrm{rt}$ for $24 \mathrm{~h} .{ }^{b}$ Yield of isolated product. ${ }^{c}$ Determined by chiral HPLC. ${ }^{d} N, N$-Dicyclphexylmethylamine. ${ }^{e} 1,8$ Diazabicyclo $[5,4,0]$ undec-7-ene. ${ }^{f}$ 4-Dimethylaminopyridine. ${ }^{g}$ The reaction time was $72 \mathrm{~h} .{ }^{h}$ Dicyclohexylamine. ${ }^{i}$ 2,2,6,6-Tetramethylpiperidine.

Table S5: The effect of the molar ratio of 1a/2a for copper/L1-mediated alkynylation of isatins. ${ }^{a}$

\begin{tabular}{cccc}
\hline Entry & The amount of 2a & Yield $(\%)^{b}$ & $e e(\%)^{c}$ \\
\hline 1 & $0.1 \mathrm{mmol}$ & 72 & $42(S)$ \\
2 & $0.2 \mathrm{mmol}$ & 94 & $78(R)$ \\
3 & $0.3 \mathrm{mmol}$ & 95 & $73(R)$ \\
4 & $0.4 \mathrm{mmol}$ & 97 & $77(R)$ \\
${ }^{a}$ Unless otherwise noted, all reactions were performed with $\mathbf{L 1} / \mathrm{CuOTf} \cdot 0.5 \mathrm{PhH}(1 / 3,10$ \\
mol\%), 1a (0.1 mmol), Et $3 \mathrm{~N}(20 \mathrm{~mol} \%)$, TFE $(1.0 \mathrm{~mL})$, at $\mathrm{rt}$ for $24 \mathrm{~h}^{b}{ }^{\text {Isolated yield. }^{c}}$ \\
Determined by HPLC.
\end{tabular}

Table S6: The effects of the molar ratio of $\mathbf{L 6} / \mathrm{CuOTf} \cdot 0.5 \mathrm{PhH}^{a}$

\begin{tabular}{ccccc}
\hline Entry & The amount of L6 & $\begin{array}{c}\text { The amount of } \\
\text { CuOTf } \cdot 0.5 \mathrm{PhH}\end{array}$ & Yield $(\%)^{b}$ & ee $(\%)^{c}$ \\
\hline 1 & $10 \mathrm{~mol} \%$ & $10 \mathrm{~mol} \%$ & trace & \\
2 & $10 \mathrm{~mol} \%$ & $20 \mathrm{~mol} \%$ & 47 & $81(R)$ \\
3 & $10 \mathrm{~mol} \%$ & $30 \mathrm{~mol} \%$ & 92 & $89(R)$ \\
4 & $10 \mathrm{~mol} \%$ & $40 \mathrm{~mol} \%$ & 80 & $82(R)$ \\
5 & $10 \mathrm{~mol} \%$ & $5 \mathrm{~mol} \%$ & trace & \\
6 & $20 \mathrm{~mol} \%$ & $10 \mathrm{~mol} \%$ & trace &
\end{tabular}

${ }^{a}$ Unless otherwise noted, all reactions were performed with $\mathbf{1 a}(0.1 \mathrm{mmol}), \mathbf{2 a}(0.2 \mathrm{mmol})$, TFE $(1.0 \mathrm{~mL}), \mathrm{Et}_{3} \mathrm{~N}(20 \mathrm{~mol} \%)$, at $\mathrm{rt}$ for $24 \mathrm{~h} .{ }^{b}$ Isolated yield. ${ }^{c}$ Determined by HPLC.

Table S7: The effect of the amount of $\mathrm{Et}_{3} \mathrm{~N}$ for copper / L6-mediated alkynylation of isatins. $^{a}$

\begin{tabular}{cccc}
\hline Entry & The amount of $\mathrm{Et}_{3} \mathrm{~N}$ & Yield $(\%)^{b}$ & $e e(\%)^{c}$ \\
\hline 1 & $5 \mathrm{~mol} \%$ & 31 & $13(S)$ \\
2 & $10 \mathrm{~mol} \%$ & 97 & $63(R)$ \\
3 & $20 \mathrm{~mol} \%$ & 92 & $89(R)$ \\
4 & $30 \mathrm{~mol} \%$ & 85 & $83(R)$ \\
5 & $40 \mathrm{~mol} \%$ & 83 & $78(R)$
\end{tabular}

${ }^{a}$ Unless otherwise noted, all reactions were performed with $\mathbf{L 6} / \mathrm{CuOTf} \cdot 0.5 \mathrm{PhH}(1 / 3,10 \mathrm{~mol} \%)$, 1a $(0.1 \mathrm{mmol}), 2 \mathrm{a}(0.2 \mathrm{mmol})$, TFE $(1.0 \mathrm{~mL})$, at $\mathrm{rt}$ for $24 \mathrm{~h} .{ }^{b}$ Isolated yield. ${ }^{c}$ Determined by 
HPLC.

Table S8: The effect of the amount of $\mathrm{H}_{2} \mathrm{O}$ for copper / L6-mediated alkynylation of isatins $^{a}$.

\begin{tabular}{cccc}
\hline Entry & The amount of $\mathrm{H}_{2} \mathrm{O}$ & Yield $(\%)^{b}$ & ee $(\%)^{c}$ \\
\hline 1 & $250 \mu \mathrm{L}$ & 65 & $92(R)$ \\
2 & $200 \mu \mathrm{L}$ & 70 & $93(R)$ \\
3 & $100 \mu \mathrm{L}$ & 74 & $92(R)$ \\
4 & $50 \mu \mathrm{L}$ & 78 & $93(R)$ \\
5 & $10 \mu \mathrm{L}$ & 90 & $95(R)$
\end{tabular}

${ }^{a}$ Unless otherwise noted, all reactions were performed with $\mathbf{L 6}$ /CuOTf $\cdot 0.5 \mathrm{PhH}(1 / 3,10 \mathrm{~mol} \%)$, 1a $(0.1 \mathrm{mmol}), \mathbf{2 a}(0.2 \mathrm{mmol}), \mathrm{Et}_{3} \mathrm{~N}(20 \mathrm{~mol} \%)$, TFE $(1.0 \mathrm{~mL})$, at $\mathrm{rt}$ for $24 \mathrm{~h}^{b}{ }^{b}$ Isolated yield. ${ }^{c}$ Determined by HPLC.

Table S9: The effect of the molar ratio of 1a/2a for copper/L6-mediated alkynylation of isatins. ${ }^{a}$

\begin{tabular}{cccc}
\hline Entry & The amount of 2a & ${\text { Yield }(\%)^{b}}^{b}$ & $e e(\%)^{c}$ \\
\hline 1 & $0.1 \mathrm{mmol}$ & 65 & $94(R)$ \\
2 & $0.12 \mathrm{mmol}$ & 75 & $93(R)$ \\
3 & $0.15 \mathrm{mmol}$ & 83 & $93(R)$ \\
4 & $0.2 \mathrm{mmol}$ & 90 & $95(R)$ \\
5 & $0.3 \mathrm{mmol}$ & 99 & $95(R)$ \\
${ }^{a}$ Unless otherwise noted, all reactions were performed with $\mathbf{L 6} / \mathrm{CuOTf} \cdot 0.5 \mathrm{PhH}(1 / 3,10 \mathrm{~mol} \%)$, \\
1a $(0.1$ mmol $)$, Et ${ }_{3} \mathrm{~N}(20 \mathrm{~mol} \%)$, TFE $(1.0 \mathrm{~mL}), \mathrm{H}_{2} \mathrm{O}(10 \mu \mathrm{L})$ at $\mathrm{rt}$ for $24 \mathrm{~h} .^{b}$ Isolated yield. ${ }^{c}$ \\
Determined by HPLC.
\end{tabular}

Table S10: The effect of the concentration of 1a for copper/L6-mediated alkynylation of isatins ${ }^{a}$.

\begin{tabular}{cccc}
\hline Entry & The amount of TFE & Yield $(\%)^{b}$ & ee $(\%)^{c}$ \\
\hline 1 & $0.5 \mathrm{~mL}$ & 99 & $91(R)$ \\
2 & $1.0 \mathrm{~mL}$ & 99 & $95(R)$ \\
3 & $1.5 \mathrm{~mL}$ & 95 & $94(R)$ \\
${ }^{a}$ Unless otherwise noted, all reactions were performed with $\mathbf{~ L 6 / C u O T f} \cdot 0.5 \mathrm{PhH}(1 / 3,10 \mathrm{~mol} \%)$, \\
$\mathbf{1 a}(0.1$ mmol $), \mathbf{2 a}(0.3 \mathrm{mmol}), \mathrm{Et}_{3} \mathrm{~N}(20 \mathrm{~mol} \%), \mathrm{H}_{2} \mathrm{O}(10 \mu \mathrm{L})$ at $\mathrm{rt}$ for $24 \mathrm{~h} .^{b}$ Isolated yield. $^{c}$ \\
Determined by HPLC.
\end{tabular}

Table S11: The effect of the base (10 mol\%) for copper/L1-mediated alkynylation of 
isatins. $^{a}$

\begin{tabular}{ccccc}
\hline Entry & Base & The amount of base & ${\text { Yield }(\%)^{b}}^{e e}(\%)^{c}$ \\
\hline 1 & $\mathrm{Et}_{3} \mathrm{~N}$ & $10 \mathrm{~mol} \%$ & 97 & $63(S)$ \\
2 & $\mathrm{Cy} 2 \mathrm{NH}$ & $10 \mathrm{~mol} \%$ & 85 & $48(S)$ \\
3 & $\mathrm{DMAP}$ & $10 \mathrm{~mol} \%$ & 40 & $52(S)$ \\
4 & $\mathrm{DBU}$ & $10 \mathrm{~mol} \%$ & 90 & $48(S)$ \\
5 & Pyridine & $10 \mathrm{~mol} \%$ & 32 & $30(S)$ \\
6 & $\mathrm{TMP}$ & $10 \mathrm{~mol} \%$ & 70 & $56(R)$ \\
7 & $i$-Pr $2 \mathrm{NEt}$ & $10 \mathrm{~mol} \%$ & 98 & $62(S)$
\end{tabular}

${ }^{a}$ Unless otherwise noted, all reactions were performed with $\mathbf{L 1} / \mathrm{CuOTf} \cdot 0.5 \mathrm{PhH}(1 / 3,10 \mathrm{~mol} \%)$, 1a $(0.1 \mathrm{mmol}), 2 \mathrm{a}(0.4 \mathrm{mmol})$, TFE $(1.0 \mathrm{~mL})$, at $\mathrm{rt}$ for $24 \mathrm{~h} .{ }^{b}$ Isolated yield. ${ }^{c}$ Determined by HPLC.

Table S11: The effect of different ligands for alkynylation of isatins in the presence of $i$-Pr 2 NEt $\left(10 \mathrm{~mol}^{\%}\right){ }^{a}$

\begin{tabular}{|c|c|c|c|c|}
\hline Entry & Base & Ligand (10 mol\%) & Yield $(\%)^{b}$ & $e e(\%)^{c}$ \\
\hline 1 & $i$-Pr ${ }_{2} \mathrm{NEt}(10 \mathrm{~mol} \%)$ & L1 & 98 & $62(S)$ \\
\hline 2 & $i$ - $\operatorname{Pr}_{2} \mathrm{NEt}(10 \mathrm{~mol} \%)$ & $\mathbf{L} 2$ & 73 & $40(S)$ \\
\hline 3 & $i$ - $\operatorname{Pr}_{2} \mathrm{NEt}(10 \mathrm{~mol} \%)$ & $\mathbf{L 3}$ & 58 & $50(S)$ \\
\hline 4 & $i$ - $\operatorname{Pr}_{2} \mathrm{NEt}(10 \mathrm{~mol} \%)$ & L4 & 73 & $70(S)$ \\
\hline 5 & $i-\operatorname{Pr}_{2} \mathrm{NEt}(10 \mathrm{~mol} \%)$ & $\mathbf{L 5}$ & 38 & $42(S)$ \\
\hline 6 & $i$ - $\operatorname{Pr}_{2} \mathrm{NEt}(10 \mathrm{~mol} \%)$ & L6 & 81 & $30(S)$ \\
\hline 7 & $i$ - $\operatorname{Pr}_{2} \mathrm{NEt}(10 \mathrm{~mol} \%)$ & L7 & 86 & $39(S)$ \\
\hline 8 & $i$ - $\operatorname{Pr}_{2} \mathrm{NEt}(10 \mathrm{~mol} \%)$ & L8 & 96 & $37(S)$ \\
\hline 9 & $i$ - $\operatorname{Pr}_{2} \mathrm{NEt}(10 \mathrm{~mol} \%)$ & L9 & 82 & $60(S)$ \\
\hline 10 & $i$ - $\operatorname{Pr}_{2} \mathrm{NEt}(10 \mathrm{~mol} \%)$ & L10 & 99 & $81(S)$ \\
\hline 11 & $i-\operatorname{Pr}_{2} \mathrm{NEt}(10 \mathrm{~mol} \%)$ & L11 & 64 & $30(S)$ \\
\hline
\end{tabular}

${ }^{a}$ Unless otherwise noted, all reactions were performed with $\mathrm{L} / \mathrm{CuOTf} \cdot 0.5 \mathrm{PhH}(1 / 3,10 \mathrm{~mol} \%)$, 1a $(0.1 \mathrm{mmol}), 2 \mathrm{a}(0.4 \mathrm{mmol})$, TFE $(1.0 \mathrm{~mL})$, at $\mathrm{rt}$ for $24 \mathrm{~h} .{ }^{b}$ Isolated yield. ${ }^{c}$ Determined by HPLC.

Table S12: The effect of the different ligands for alkynylation of isatins in the presence of $\mathrm{Et}_{3} \mathrm{~N}(10 \mathrm{~mol} \%){ }^{a}$

\begin{tabular}{ccccc}
\hline Entry & Base & Ligand & ${\text { Yield }(\%)^{b}}$ & $e e(\%)^{c}$ \\
\hline 1 & Et $_{3} \mathrm{~N}(10 \mathrm{~mol} \%)$ & L1 & 97 & $63(S)$ \\
2 & Et $_{3} \mathrm{~N}(10 \mathrm{~mol} \%)$ & L2 & 45 & $47(S)$ \\
3 & Et $_{3} \mathrm{~N}(10 \mathrm{~mol} \%)$ & L3 & 81 & $29(S)$
\end{tabular}




$\begin{array}{ccccc}4 & \mathrm{Et}_{3} \mathrm{~N}(10 \mathrm{~mol} \%) & \mathbf{L 4} & 78 & 57(S) \\ 5 & \mathrm{Et} \mathrm{t}_{3} \mathrm{~N}(10 \mathrm{~mol} \%) & \mathbf{L 5} & 28 & 13(S) \\ 6 & \mathrm{Et} \mathrm{t}_{3} \mathrm{~N}(10 \mathrm{~mol} \%) & \mathbf{L 6} & 99 & 67(R) \\ 7 & \mathrm{Et} \mathrm{t}_{3} \mathrm{~N}(10 \mathrm{~mol} \%) & \mathbf{L 7} & 86 & 75(R) \\ 8 & \mathrm{Et} \mathrm{t}_{3} \mathrm{~N}(10 \mathrm{~mol} \%) & \mathbf{L 8} & 63 & 15(R) \\ 9 & \mathrm{Et}_{3} \mathrm{~N}(10 \mathrm{~mol} \%) & \mathbf{L 9} & 90 & 38(S) \\ 10 & \mathrm{Et}_{3} \mathrm{~N}(10 \mathrm{~mol} \%) & \mathbf{L 1 0} & 92 & 78(S) \\ 11 & \mathrm{Et}_{3} \mathrm{~N}(10 \mathrm{~mol} \%) & \mathbf{L 1 1} & 46 & 41(S)\end{array}$

${ }^{a}$ Unless otherwise noted, all reactions were performed with $\mathbf{L} / \mathrm{CuOTf} \cdot 0.5 \mathrm{PhH}(1 / 3,10 \mathrm{~mol} \%)$, 1a $(0.1 \mathrm{mmol}), 2 \mathrm{a}(0.4 \mathrm{mmol})$, TFE $(1.0 \mathrm{~mL})$, at $\mathrm{rt}$ for $24 \mathrm{~h} .{ }^{b}$ Isolated yield. ${ }^{c}$ Determined by HPLC.

Table S13: The effect of the base for copper/L10-mediated alkynylation of isatin. ${ }^{a}$

\begin{tabular}{ccccc}
\hline Entry & Base & The amount of Base & Yield $(\%)^{b}$ & $e e(\%)^{c}$ \\
\hline 1 & $\mathrm{Et}_{3} \mathrm{~N}$ & $20 \mathrm{~mol} \%$ & 99 & $80(R)$ \\
2 & $i$-Pr${ }_{2} \mathrm{NEt}$ & $5 \mathrm{~mol} \%$ & 63 & $65(S)$ \\
3 & $i-\mathrm{Pr}_{2} \mathrm{NEt}$ & $10 \mathrm{~mol} \%$ & 99 & $81(S)$ \\
4 & $i$-Pr${ }_{2} \mathrm{NEt}$ & $20 \mathrm{~mol} \%$ & 97 & $35(R)$ \\
5 & $i-\mathrm{Pr}_{2} \mathrm{NEt}$ & $30 \mathrm{~mol} \%$ & 81 & $45(R)$ \\
6 & $\mathrm{Cy}_{2} \mathrm{NH}$ & $20 \mathrm{~mol} \%$ & 99 & $67(S)$ \\
7 & $\mathrm{DMAP}$ & $20 \mathrm{~mol} \%$ & 84 & $32(S)$ \\
8 & $\mathrm{DBU}$ & $20 \mathrm{~mol} \%$ & 82 & $64(R)$ \\
9 & $\mathrm{Pyridine}$ & $20 \mathrm{~mol} \%$ & 76 & $53(S)$ \\
10 & $\mathrm{TMP}$ & $20 \mathrm{~mol} \%$ & 80 & $60(R)$
\end{tabular}

${ }^{a}$ Unless otherwise noted, all reactions were performed with $\mathbf{L 1 0} / \mathrm{CuOTf} \cdot 0.5 \mathrm{PhH}(1 / 3,10 \mathrm{~mol} \%)$, 1a $(0.1 \mathrm{mmol}), 2 \mathrm{a}(0.4 \mathrm{mmol})$, TFE $(1.0 \mathrm{~mL})$, at $\mathrm{rt}$ for $24 \mathrm{~h} .{ }^{b}$ Isolated yield. ${ }^{c}$ Determined by HPLC.

Table S14: The effects of the molar ratio of $\mathbf{L 1 0} / \mathrm{CuOTf} \cdot 0.5 \mathrm{PhH}^{a}$

\begin{tabular}{ccccc}
\hline Entry & The amount of L10 & $\begin{array}{c}\text { The amount of } \\
\text { CuOTf } \cdot 0.5 \mathrm{PhH}\end{array}$ & Yield $(\%)^{b}$ & $e e(\%)^{c}$ \\
\hline 1 & $10 \mathrm{~mol} \%$ & $10 \mathrm{~mol} \%$ & trace & - \\
2 & $10 \mathrm{~mol} \%$ & $20 \mathrm{~mol} \%$ & 49 & $82(R)$ \\
3 & $10 \mathrm{~mol} \%$ & $30 \mathrm{~mol} \%$ & 99 & $81(S)$ \\
${ }^{a}$ Unless otherwise noted, all reactions were performed with $\mathbf{1 a}(0.1 \mathrm{mmol}), \mathbf{2 a}(0.4 \mathrm{mmol}), \mathrm{TFE}$ \\
$(1.0 \mathrm{~mL}), i-\mathrm{Pr}_{2} \mathrm{NEt}(10 \mathrm{~mol} \%)$, at rt for $24 \mathrm{~h}^{b}$ Isolated yield. ${ }^{c}$ Determined by HPLC.
\end{tabular}

Table S15: The effect of the molar ratio of $\mathbf{1 a} / \mathbf{2 a}$ for copper/L10-mediated alkynylation 
of isatins. ${ }^{a}$

\begin{tabular}{cccc}
\hline Entry & The amount of $\mathbf{2 a}$ & ${\text { Yield }(\%)^{b}}^{b}$ & ee (\%) \\
\hline 1 & $0.1 \mathrm{mmol}$ & 52 & $68(S)$ \\
2 & $0.2 \mathrm{mmol}$ & 86 & $75(S)$ \\
3 & $0.3 \mathrm{mmol}$ & 90 & $80(S)$ \\
4 & $0.4 \mathrm{mmol}$ & 99 & $81(S)$
\end{tabular}

${ }^{a}$ Unless otherwise noted, all reactions were performed with $\mathbf{L 1 0} / \mathrm{CuOTf} \cdot 0.5 \mathrm{PhH}(1 / 3,10$ mol\%), 1a (0.1 mmol) $i$-Pr ${ }_{2} \mathrm{NEt}(10 \mathrm{~mol} \%)$, TFE $(1.0 \mathrm{~mL})$, at $\mathrm{rt}$ for $24 \mathrm{~h}^{b}{ }^{b}$ Isolated yield. ${ }^{c}$ Determined by HPLC.

Table S16: The effect of the amount of $\mathrm{H}_{2} \mathrm{O}$ for copper/L10-mediated alkynylation of isatins. $^{a}$

\begin{tabular}{|c|c|c|c|}
\hline Entry & The amount of $\mathrm{H}_{2} \mathrm{O}$ & Yield $(\%)^{b}$ & $e e(\%)^{c}$ \\
\hline 1 & $100 \mu \mathrm{L}$ & 73 & $84(S)$ \\
\hline 2 & $50 \mu \mathrm{L}$ & 85 & $88(S)$ \\
\hline 3 & $10 \mu \mathrm{L}$ & 99 & $86(S)$ \\
\hline
\end{tabular}

Table S17: The effect of the concentration of 1a for copper/L10-mediated alkynylation of isatins. ${ }^{a}$

\begin{tabular}{cccc}
\hline Entry & The amount of TFE & ${\text { Yield }(\%)^{b}}^{b}$ & $e e(\%)^{c}$ \\
\hline 1 & $0.5 \mathrm{~mL}$ & 99 & $82(S)$ \\
2 & $1.0 \mathrm{~mL}$ & 99 & $86(S)$ \\
3 & $1.5 \mathrm{~mL}$ & 92 & $88(S)$
\end{tabular}

${ }^{a}$ Unless otherwise noted, all reactions were performed with $\mathbf{L 1 0}$ / CuOTf $\cdot 0.5 \mathrm{PhH}(1 / 3,10 \mathrm{~mol} \%)$, 1a $(0.1 \mathrm{mmol}), 2 \mathrm{a}(0.4 \mathrm{mmol}), i-\mathrm{Pr}_{2} \mathrm{NEt}(10 \mathrm{~mol} \%), \mathrm{H}_{2} \mathrm{O}(10 \mu \mathrm{L})$ at $\mathrm{rt}$ for $24 \mathrm{~h}^{b}{ }^{b}$ Isolated yield. ${ }^{c}$ Determined by HPLC.

\section{ESI-MS analysis of the complexes}

Sample preparation: CuOTf $\cdot 0.5 \mathrm{PhH}(0.01 \mathrm{mmol}$ or $0.02 \mathrm{mmol}$ or $0.03 \mathrm{mmol})$ and the ligand ( $\mathbf{L 6}$ or $\mathbf{L 1 0}, 0.01 \mathrm{mmol})$ were stirred in TFE $(0.5 \mathrm{~mL})$ at $35^{\circ} \mathrm{C}$ for $0.5 \mathrm{~h}$. The mixture was diluted with $\mathrm{MeOH}$ and injected for analysis.

4.1 ESI-MS analysis of the complexes (Instrument: Waters Acquity SQD) 


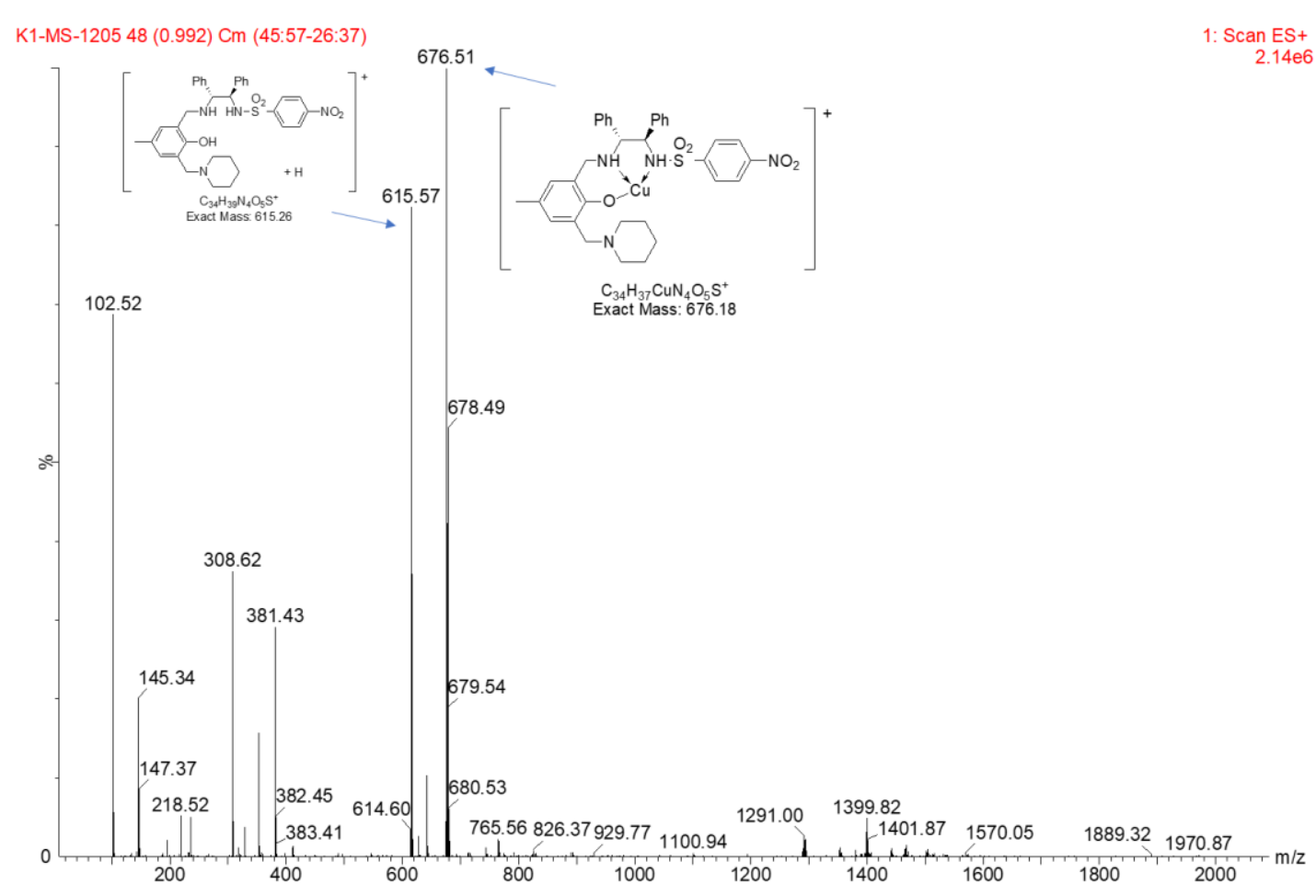

Figure S1. ESI-HRMS of CuOTf / L6 = 1:1 mixture

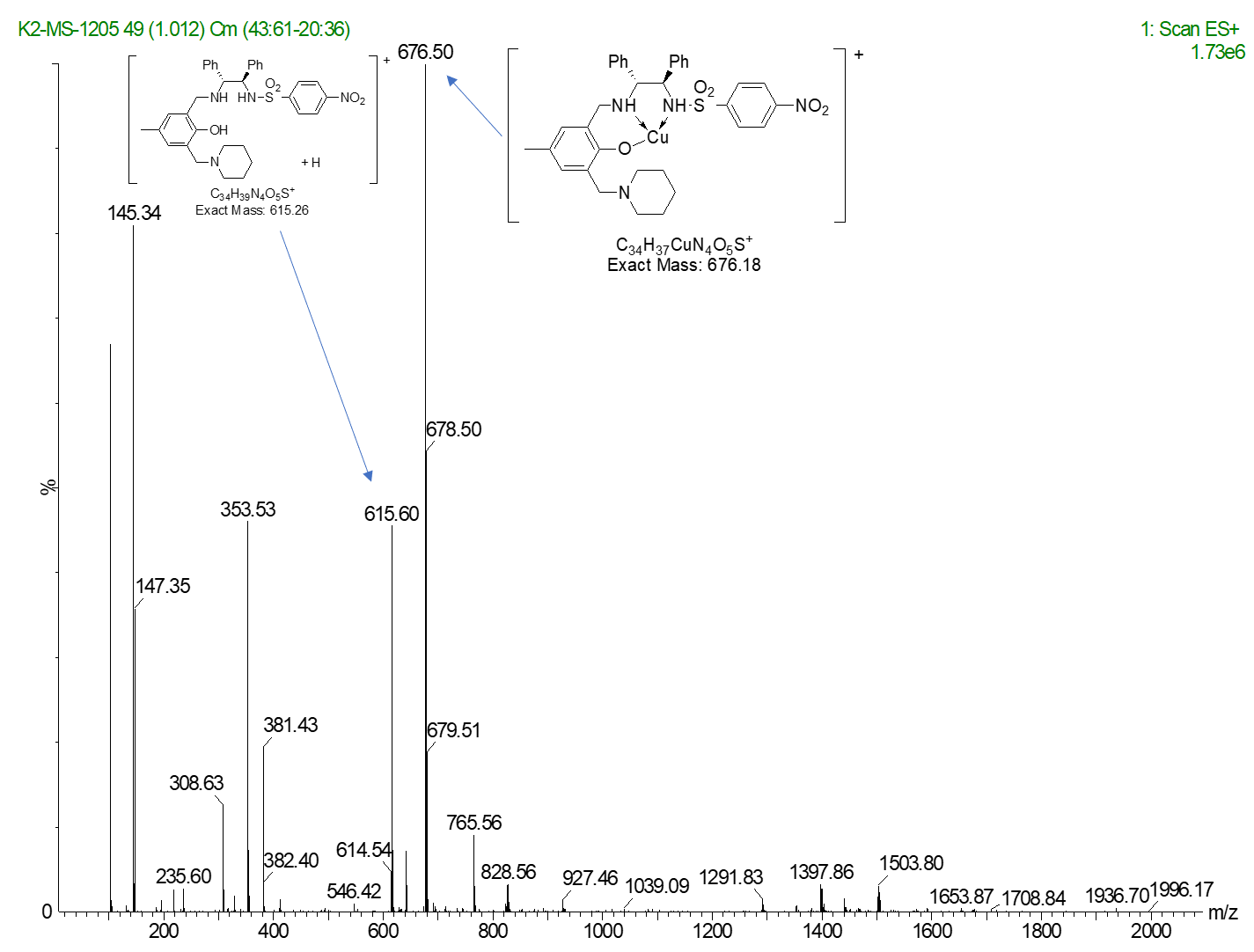

Figure S2. ESI-HRMS of CuOTf / L6 = 2:1 mixture 


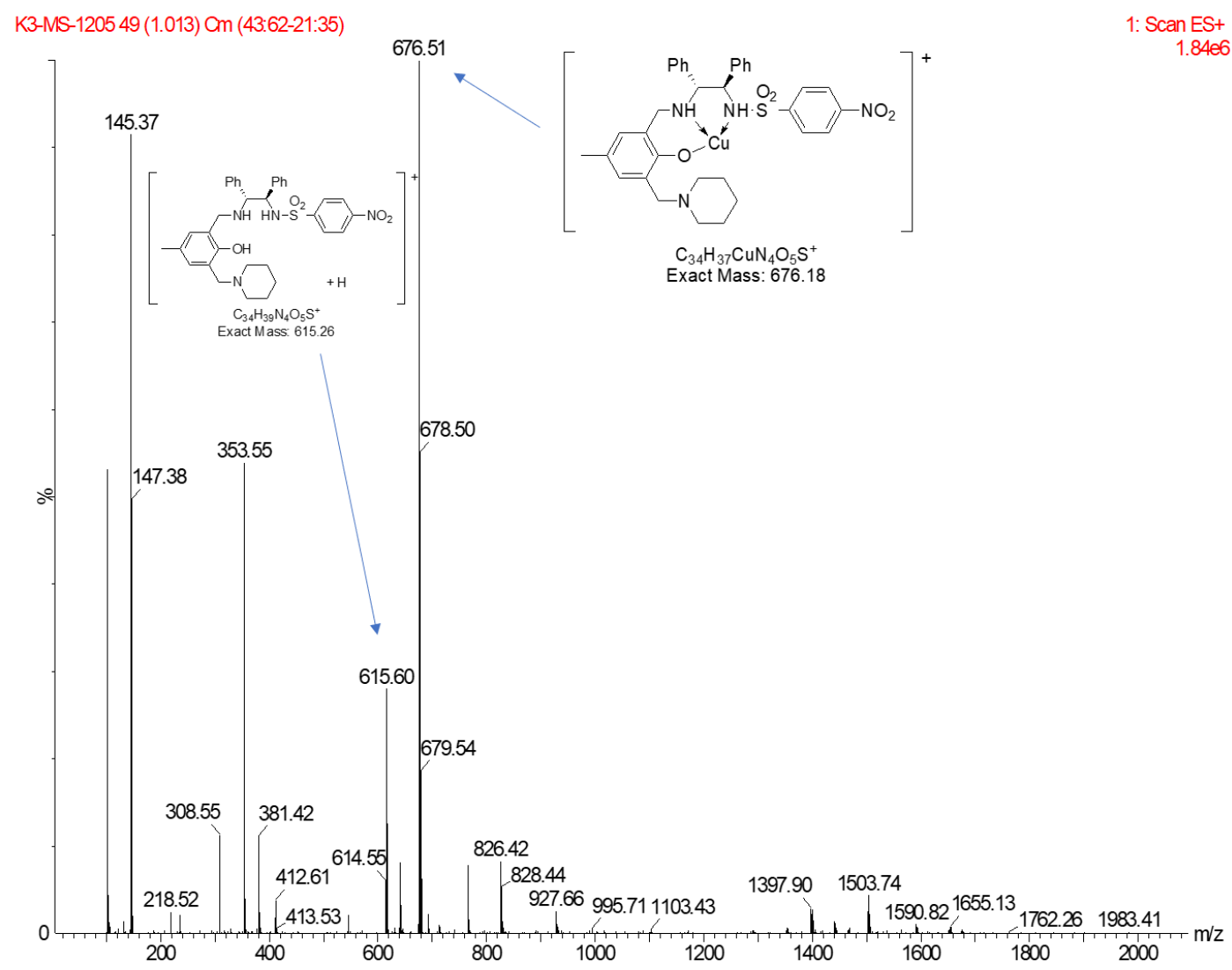

Figure S3. ESI-HRMS of CuOTf / L6 = 3:1 mixture

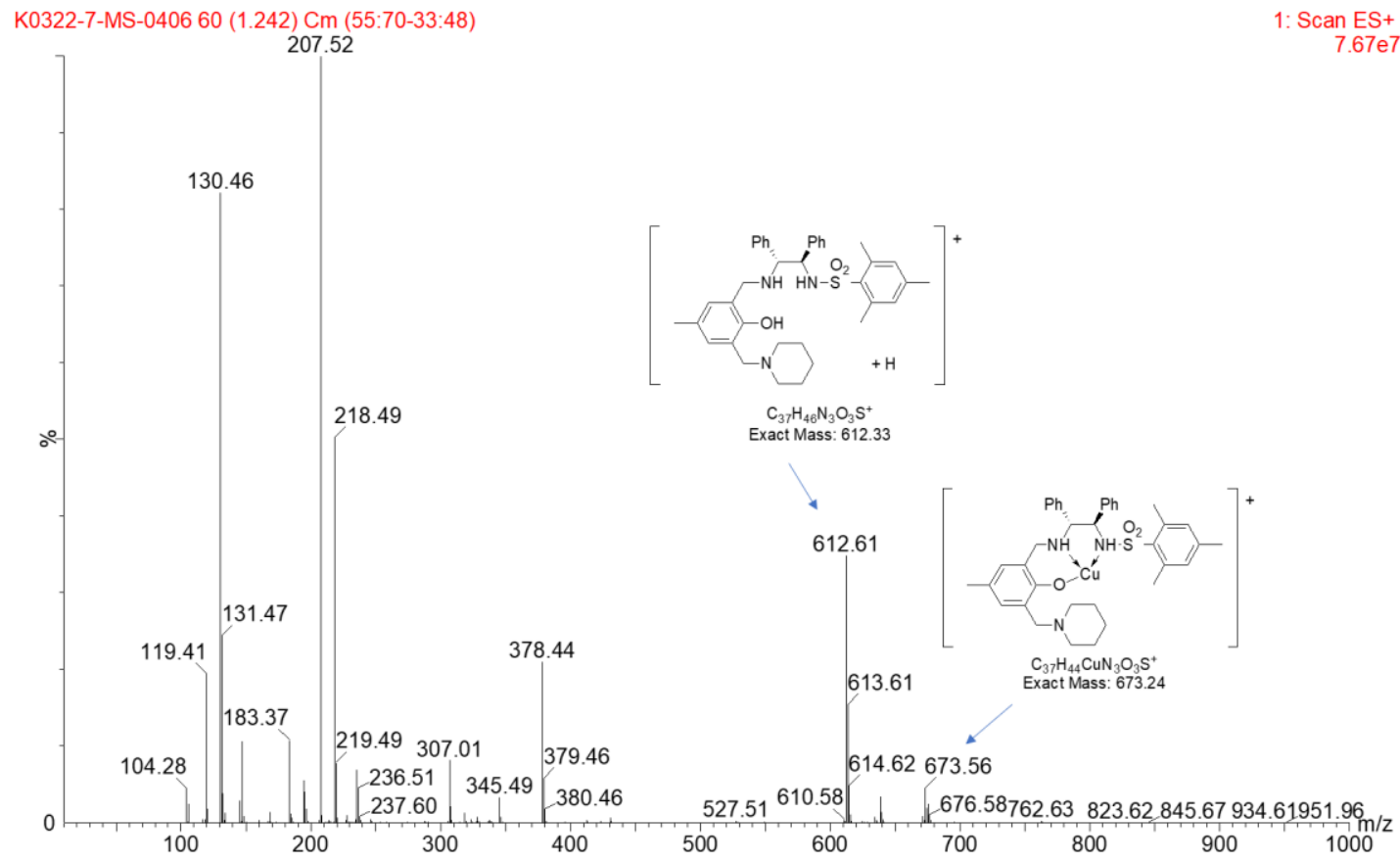

Figure S4. ESI-HRMS of CuOTf / L10 = 1:1 mixture 


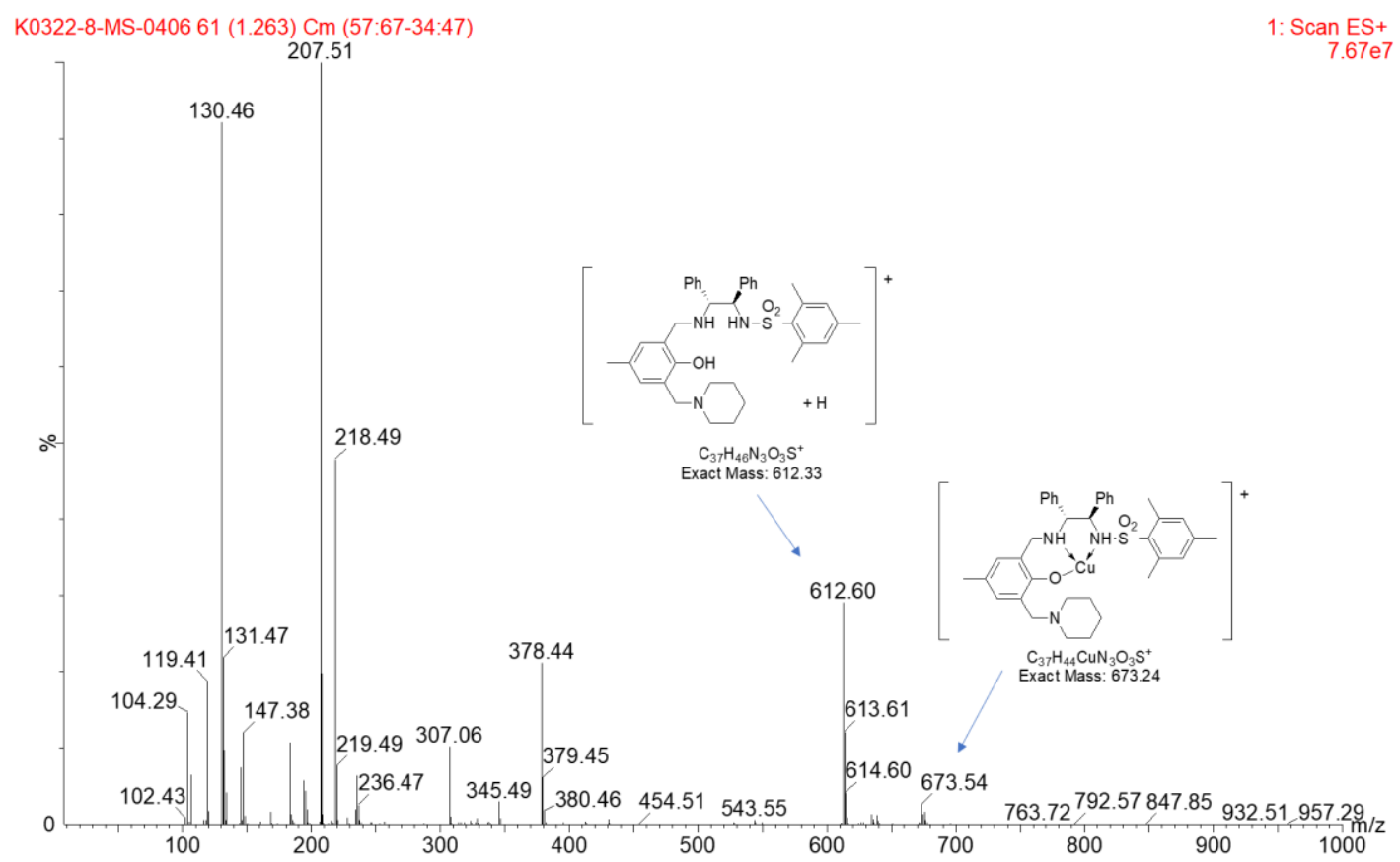

Figure S5. ESI-HRMS of CuOTf / L10 = 2:1 mixture

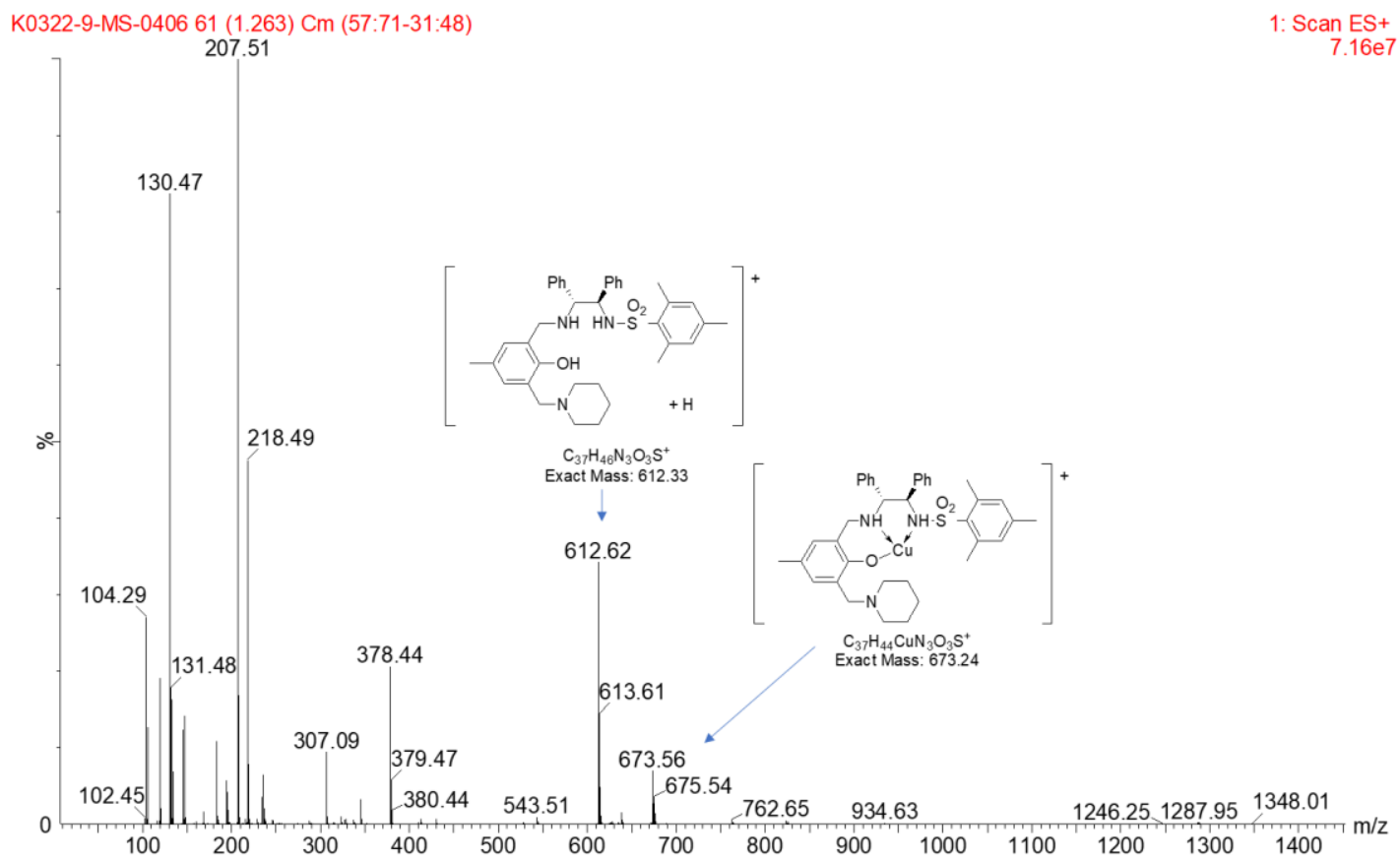

Figure S6. ESI-HRMS of CuOTf / L10 = 3:1 mixture 
4.2 ESI-HRMS analysis of the complexes (Instrument: Bruker SolariX (FT-ICR-MS))

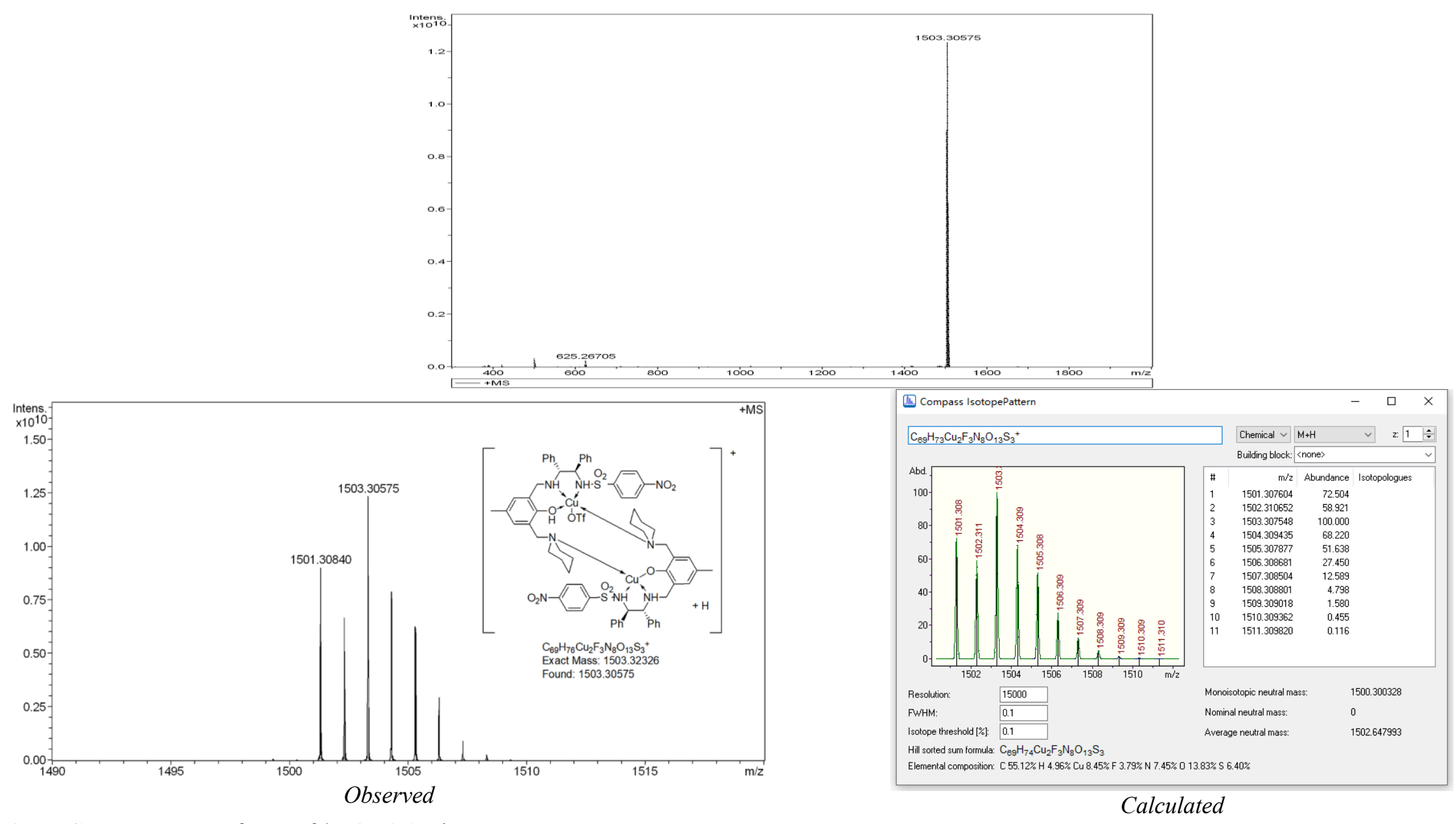

Figure S7. ESI-HRMS of CuOTf $/ \mathbf{L} \mathbf{6}=1: 1$ mixture 

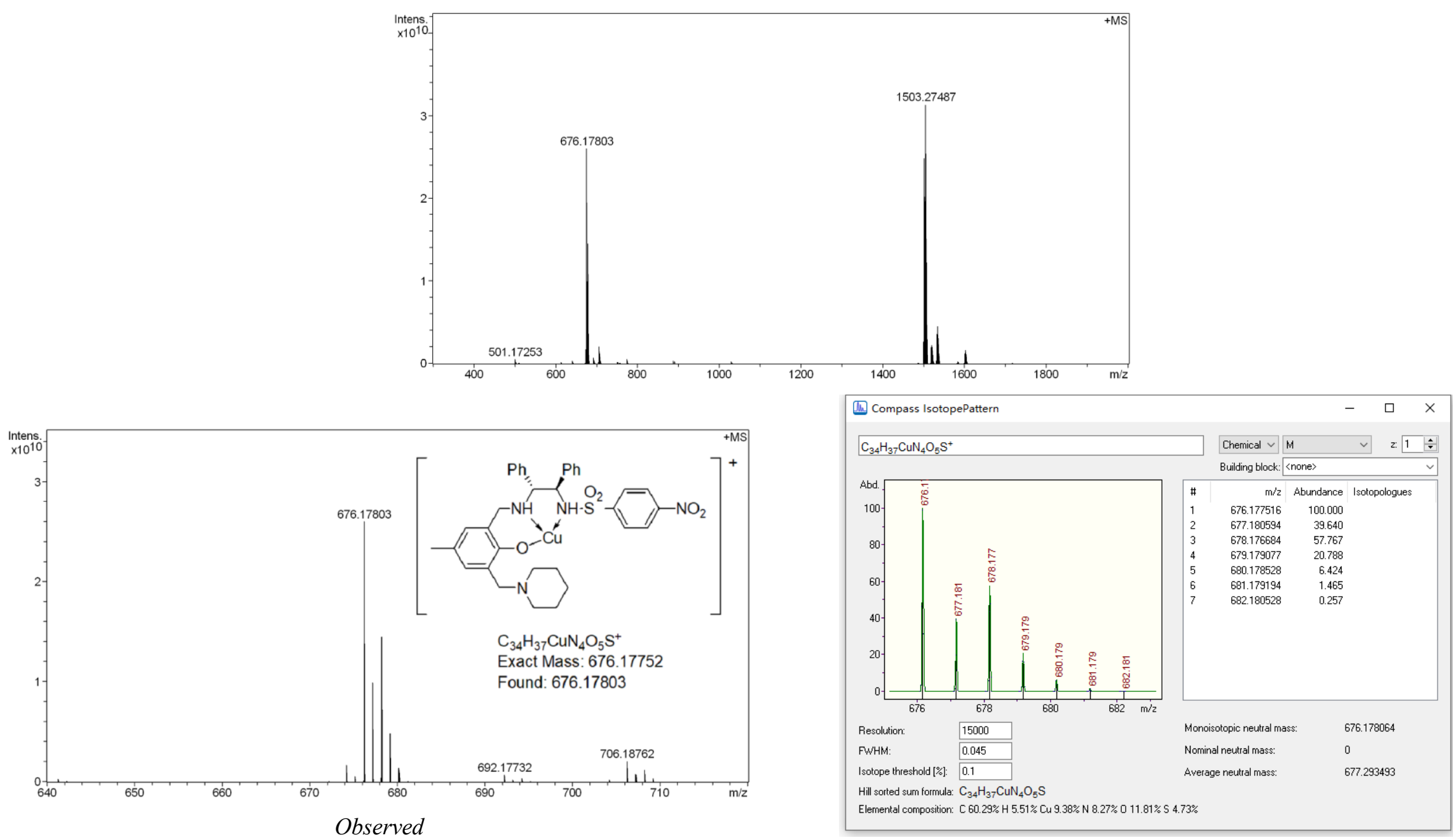

Calculated 

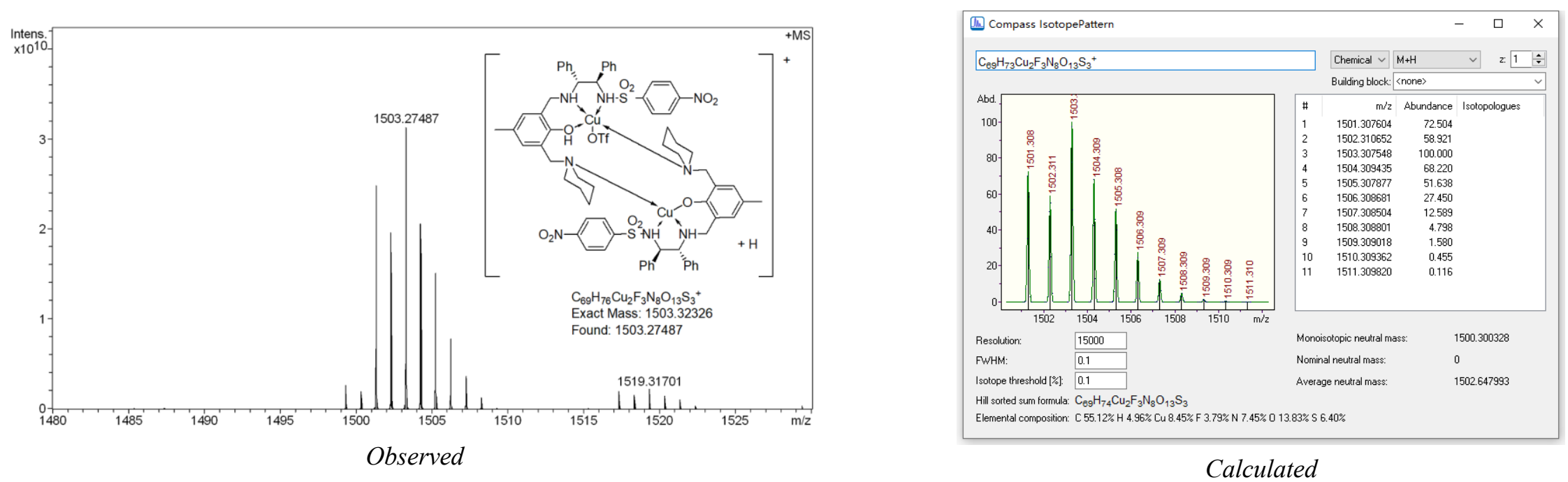

Figure S8. ESI-HRMS of CuOTf $/ \mathbf{L} \mathbf{6}=2: 1$ mixture 


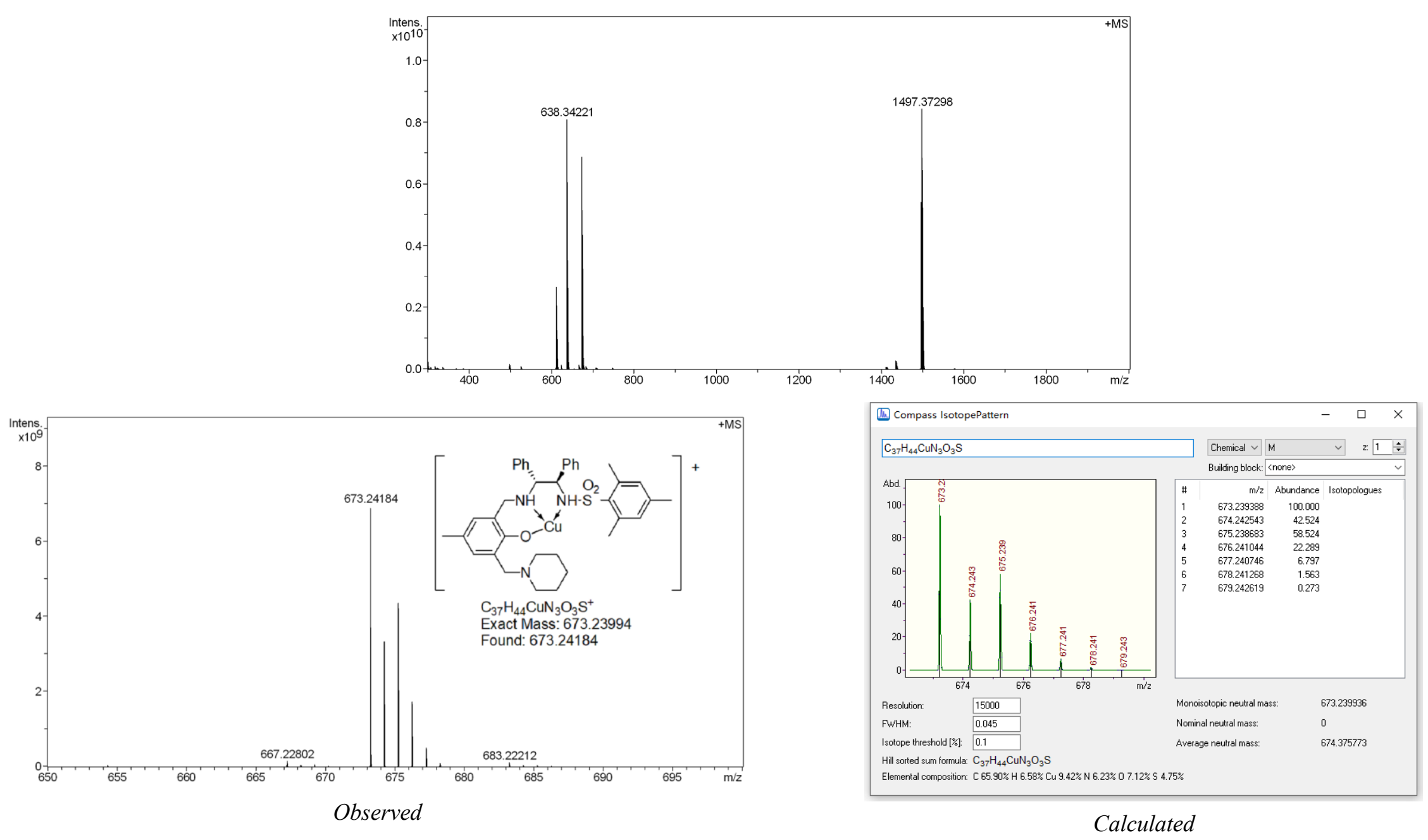




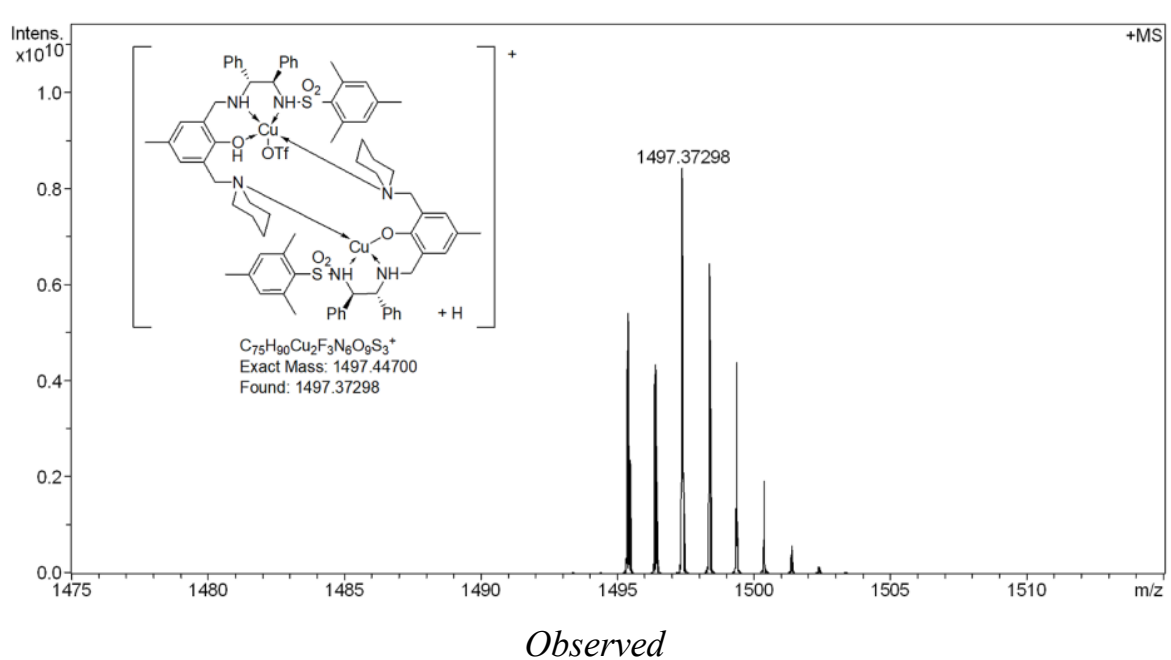

Figure S9. ESI-HRMS of CuOTf $/ \mathbf{L 1 0}=1: 1$ mixture

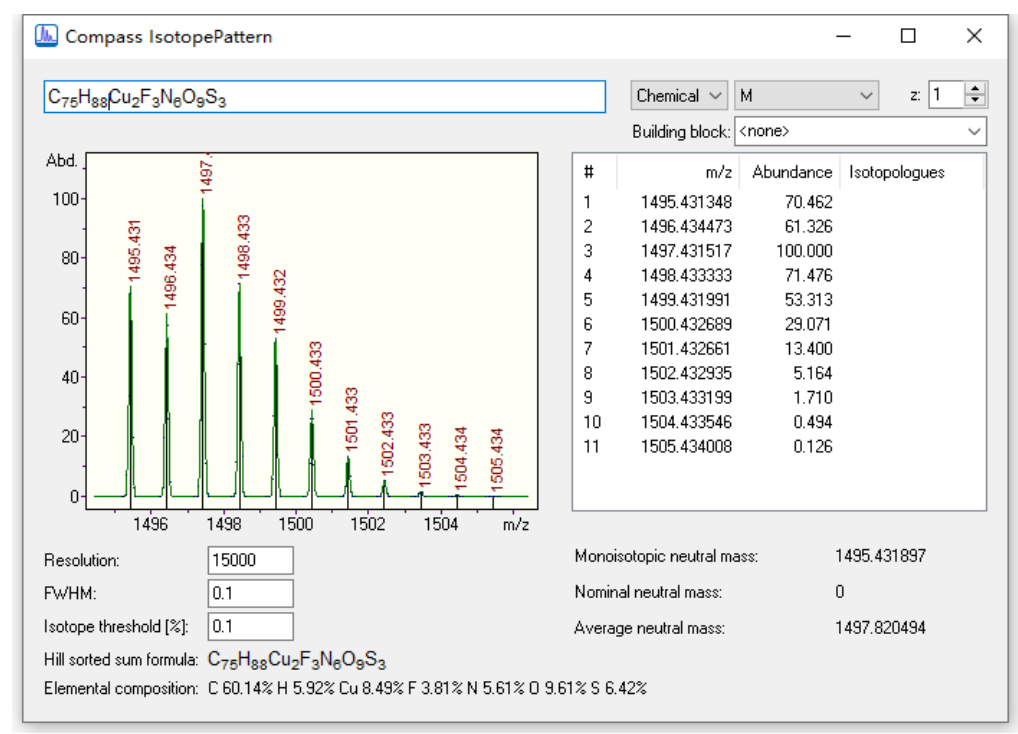

Calculated 


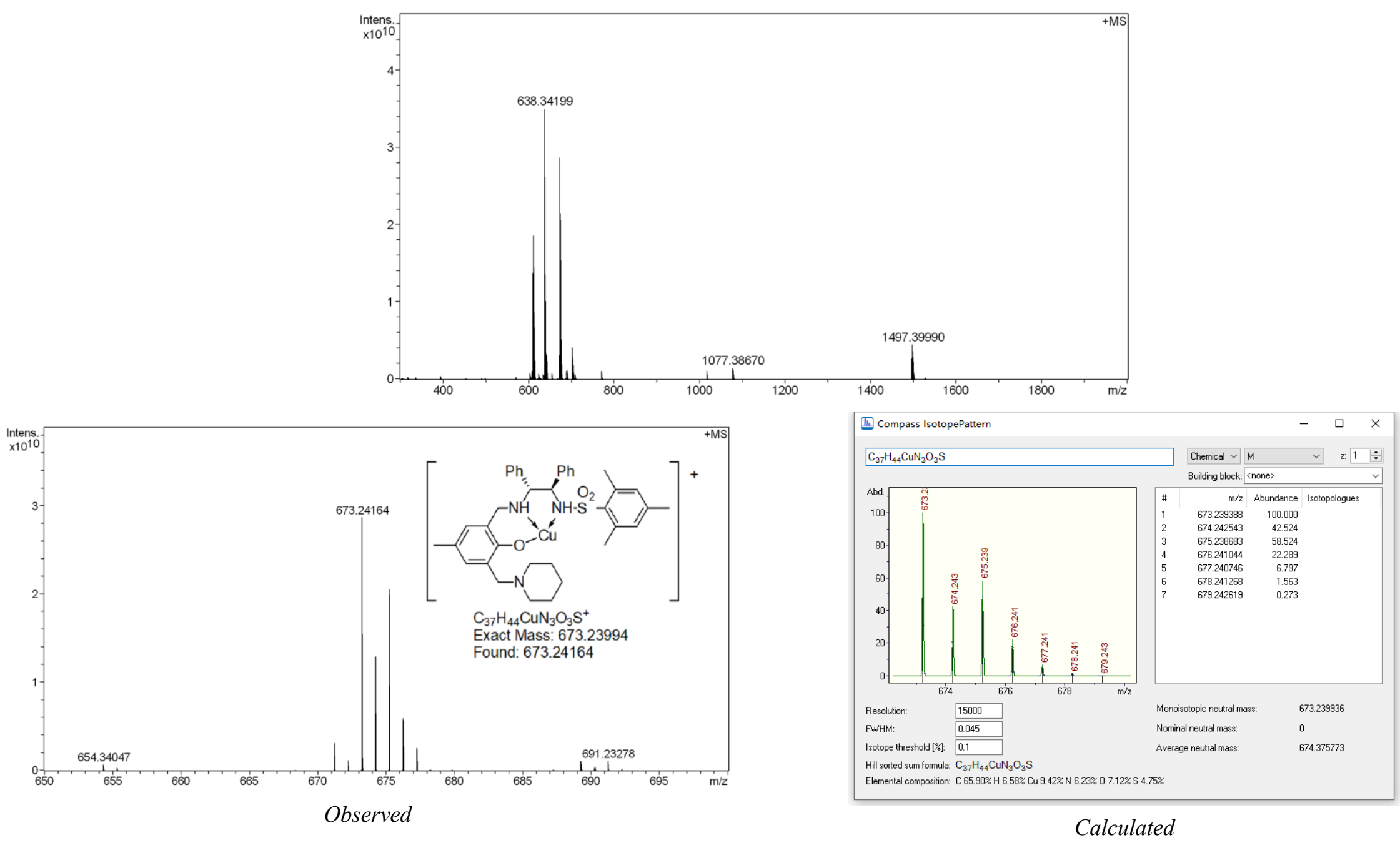




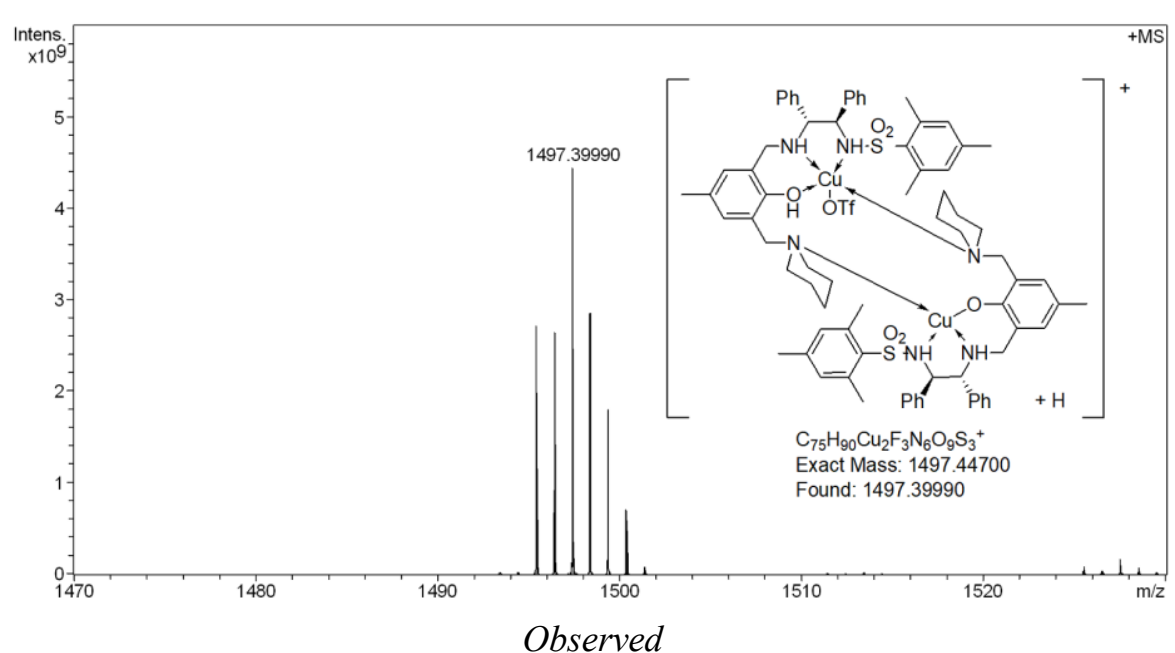

Figure S10. ESI-HRMS of CuOTf $/ \mathbf{L 1 0}=2: 1$ mixture

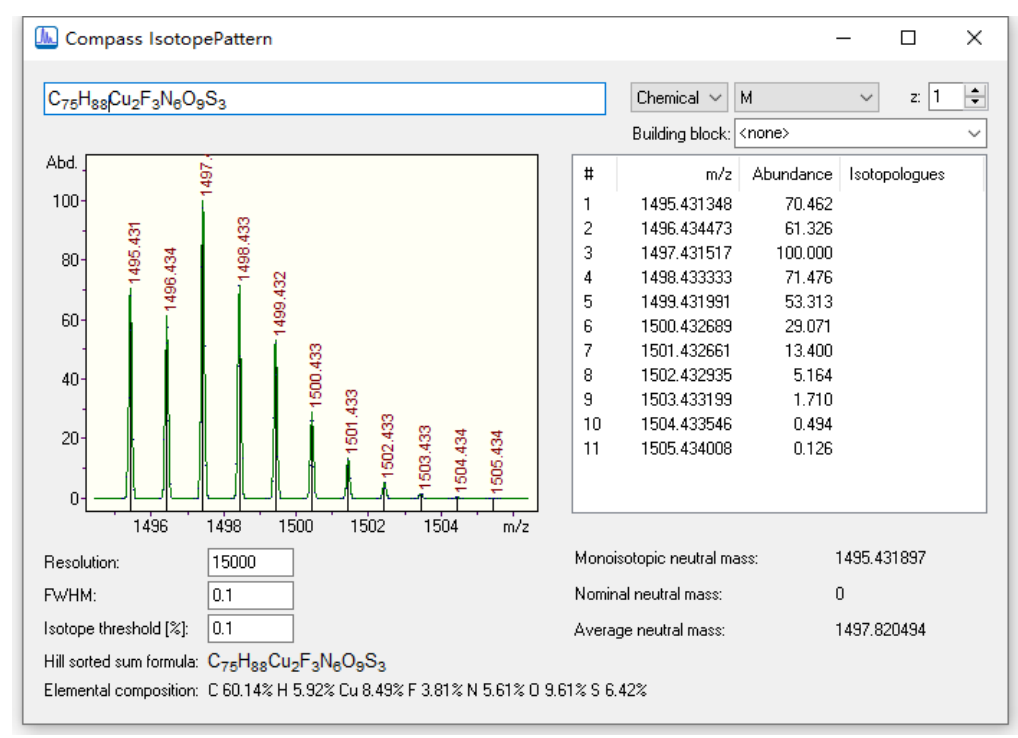

Calculated 


\section{Proposed catalytic cycle}

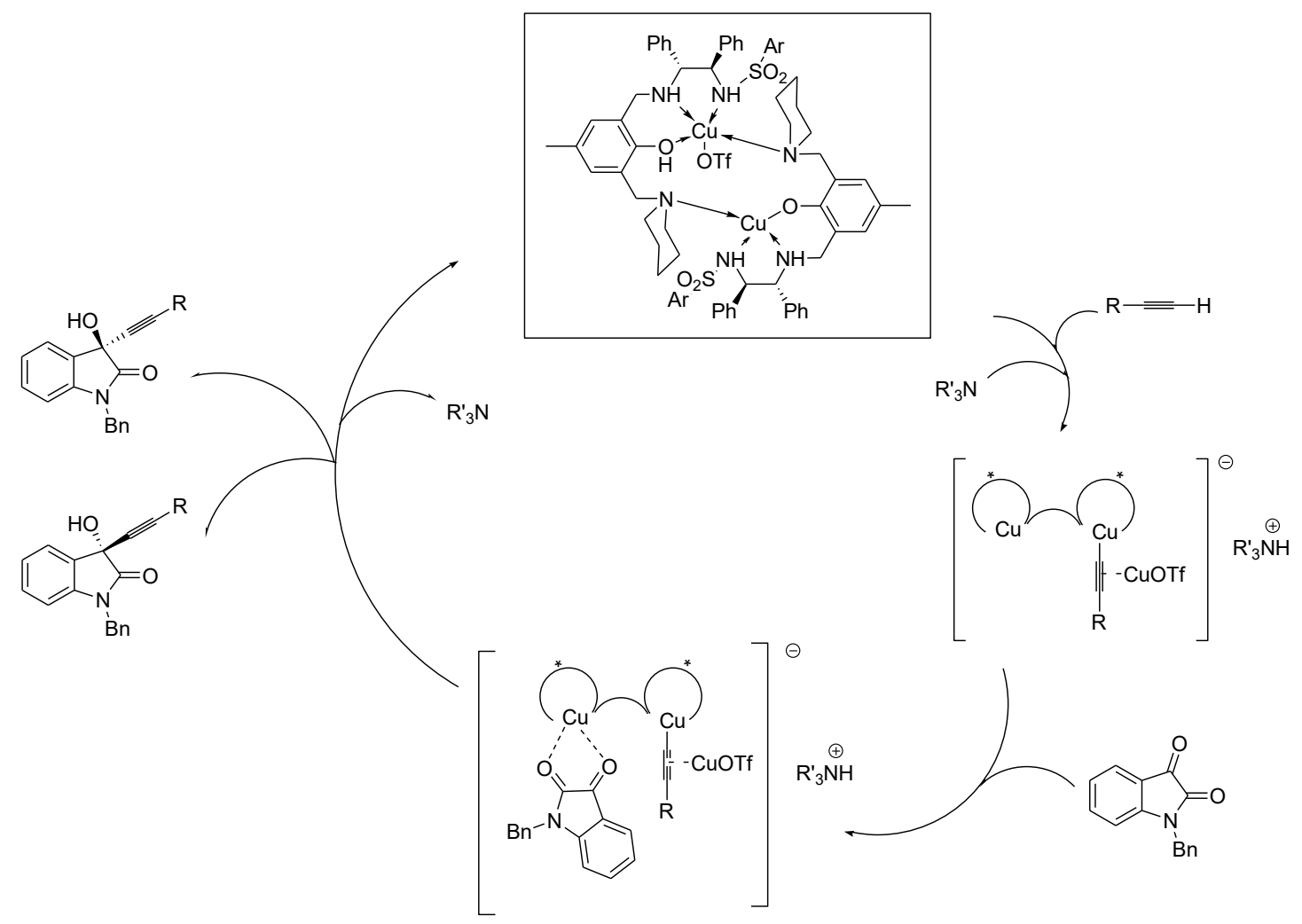

\section{General procedure for the asymmetric alkynylation of isatins}

\subsection{General procedure for the copper/L6-mediated asymmetric alkynylation of isatins.}

A mixture of $\mathbf{L 6}$ (10 mol\%), CuOTf $\cdot 0.5 \mathrm{PhH}$ (30 mol\%), Et $3 \mathrm{~N}(20 \mathrm{~mol} \%)$ was weighted into a test tube, then $0.5 \mathrm{~mL}$ solvent was added and the mixture was stirred for $30 \mathrm{~min}$ at $35^{\circ} \mathrm{C}$, followed by the additions of isatins $1(0.10 \mathrm{mmol})$ and $0.3 \mathrm{~mL}$ of solvent, the mixture was stirred for 20 minutes at room temperature, followed by the additions of alkynes $2(0.30 \mathrm{mmol})$ and $0.2 \mathrm{~mL}$ of solvent, finally the mixture was stirred for 24 hours at room temperature. The solvent was removed in vacuo and a white product can be obtained by silica gel column chromatography (dichloromethane/ethyl acetate $=50: 1$ to $30: 1)$.

\section{Scaled-up version of the reaction.}

A mixture of L6 (10 mol\%), CuOTf $\cdot 0.5 \mathrm{PhH}$ (30 mol\%), Et $3 \mathrm{~N}\left(20 \mathrm{~mol}^{\%}\right)$ was weighted into a test tube, then $15 \mathrm{~mL}$ solvent was added and the mixture was stirred for $30 \mathrm{~min}$ at $35^{\circ} \mathrm{C}$, followed by the additions of $1 \mathbf{a}(3.0 \mathrm{mmol})$ and $10 \mathrm{~mL}$ of solvent, the mixture was stirred for 20 minutes at room temperature, followed by the additions of $\mathbf{2 a}(9.0 \mathrm{mmol})$ and $5 \mathrm{~mL}$ of solvent, finally the mixture was stirred for 24 hours at room 
temperature. The solvent was removed in vacuo and a white product $\boldsymbol{R}-\mathbf{3 a a}$ can be obtained by silica gel column chromatography (dichloromethane/ethyl acetate $=50: 1$ )

6.2 General procedure for the copper/L10-mediated asymmetric alkynylation of isatins.

A mixture of L10 (10 mol\%), CuOTf $\cdot 0.5 \mathrm{PhH}$ (30 mol\%), $i$-Pr $2 \mathrm{NEt}(10 \mathrm{~mol} \%)$ was weighted into a test tube, then $0.5 \mathrm{~mL}$ solvent was added and the mixture was stirred for $30 \mathrm{~min}$ at $35^{\circ} \mathrm{C}$, followed by the additions of isatins $1(0.10 \mathrm{mmol})$ and $0.3 \mathrm{~mL}$ of solvent, the mixture was stirred for 20 minutes at room temperature, followed by the additions of alkynes $2(0.40 \mathrm{mmol})$ and $0.2 \mathrm{~mL}$ of solvent, finally the mixture was stirred for 24 hours at room temperature. The solvent was removed in vacuo and a white product can be obtained by silica gel column chromatography (dichloromethane/ethyl acetate $=50: 1$ to $30: 1)$.

\section{Scaled-up version of the reaction}

A mixture of L10 (10 mol\%), CuOTf $0.5 \mathrm{PhH}$ (30 mol\%), $i$-Pr $2 \mathrm{NEt}$ (10 mol\%) was weighted into a test tube, then $15 \mathrm{~mL}$ solvent was added and the mixture was stirred for $30 \mathrm{~min}$ at $35^{\circ} \mathrm{C}$, followed by the additions of $1 \mathrm{a}(3.0 \mathrm{mmol})$ and $10 \mathrm{~mL}$ of solvent, the mixture was stirred for 20 minutes at room temperature, followed by the additions of 2a (12 mmol) and $5 \mathrm{~mL}$ of solvent, finally the mixture was stirred for 24 hours at room temperature. The solvent was removed in vacuo and a white product $\boldsymbol{S}$-3aa can be obtained by silica gel column chromatography (dichloromethane/ethyl acetate $=50: 1$ ) 


\section{Characterization of products}

The absolute configuration of 3aa, 3ca, 3fa, 3ga, 3ia, 3ka, 3ma-3pa, 3ab-3ac, 3ae3ai, 3ak, and 3am was determined by the comparison of optical rotations with the literature data. ${ }^{1}$ The absolute stereochemistry of 3ba, 3da, 3ea, 3ha, 3ja, 3la, 3ad, 3aj, and 3al was assigned by analogy. Compounds 3aa-3ca, 3ea-3ga, 3ia, 3ka, 3ma-3pa, 3ab-3ai, 3ak, and 3am are known compounds. ${ }^{1,2,3}$

\section{1-benzyl-3-hydroxy-3-(phenylethynyl) indolin-2-one (3aa):}

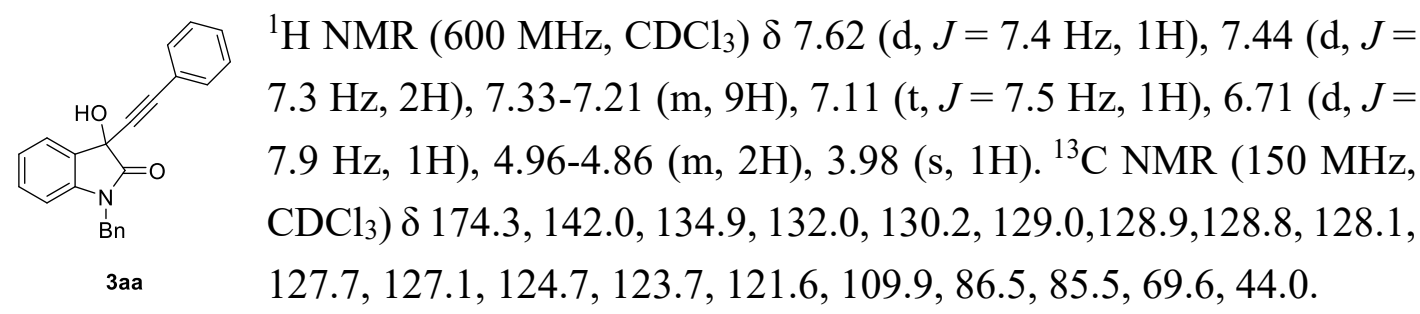

$(R)$-3aa, white solid, $33.6 \mathrm{mg}, 99 \%$ yield (dichloromethane/ethyl acetate $=50: 1$ ), 95\% $e e,[\alpha]_{\mathrm{D}}^{26}=-12.8\left(\mathrm{c}=0.59, \mathrm{CH}_{2} \mathrm{Cl}_{2}\right)$; HPLC: Daicel Chiralpak IA, n-hexane/2-propanol $=85 / 15$, flow rate $=1.0 \mathrm{~mL} / \mathrm{min}, \lambda=254 \mathrm{~nm}$, retention time: $13.0 \mathrm{~min}$ (major), 15.7 $\min ($ minor);

(S)-3aa, white solid, $33.8 \mathrm{mg}, 99 \%$ yield (dichloromethane/ethyl acetate $=50: 1$ ), 86\% $e e,[\alpha]_{\mathrm{D}}^{26}=+8.8\left(\mathrm{c}=0.62, \mathrm{CH}_{2} \mathrm{Cl}_{2}\right)\left(\operatorname{ref} .1:(S), 92 \% e e,[\alpha]_{\mathrm{D}}^{20}=+15.8(\mathrm{c}=0.54\right.$ in $\mathrm{CH}_{2} \mathrm{Cl}_{2}$ )); HPLC: Daicel Chiralpak IA, n-hexane/2-propanol $=85 / 15$, flow rate $=1.0$ $\mathrm{mL} / \mathrm{min}, \lambda=254 \mathrm{~nm}$, retention time: $13.6 \mathrm{~min}$ (minor), $16.5 \mathrm{~min}$ (major).

$(R)$-3aa (gram), white solid, $0.99 \mathrm{~g}, 97 \%$ yield (dichloromethane/ethyl acetate $=50: 1$ ), 93\% ee; HPLC: Daicel Chiralpak IA, n-hexane/2-propanol $=85 / 15$, flow rate $=1.0$ $\mathrm{mL} / \mathrm{min}, \lambda=254 \mathrm{~nm}$, retention time: $12.7 \mathrm{~min}$ (major), $15.6 \mathrm{~min}$ (minor);

(S)-3aa (gram), white solid, $0.92 \mathrm{~g}, 90 \%$ yield (dichloromethane/ethyl acetate $=50: 1$ ), 90\% ee; HPLC: Daicel Chiralpak IA, n-hexane/2-propanol $=85 / 15$, flow rate $=1.0$ $\mathrm{mL} / \mathrm{min}, \lambda=254 \mathrm{~nm}$, retention time: $13.0 \mathrm{~min}$ (minor), $15.8 \mathrm{~min}$ (major).
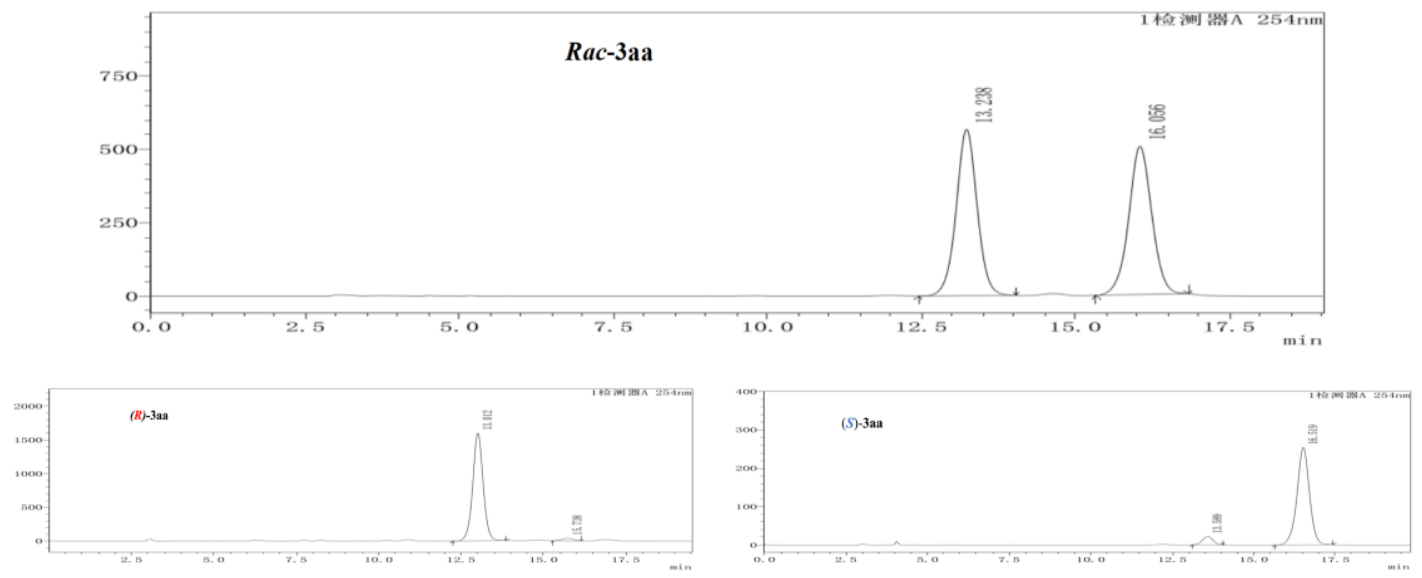

[1] Chen, L.; Huang, G.; Liu, M.; Huang, Z.; Chen, F. Adv. Synth. Catal. 2018, 360, 3497-3501.

[2] Dhara, K.; Kapat, A.; Ghosh, T.; Dash, J. Synthesis, 2016, 48, 4260-4268.

[3] Chen, J.; Li, C. Org. Lett. 2020, 22, 4686-4691. 


\begin{tabular}{|c|c|c|c|c|c|c|c|}
\hline & Retention Time & Area & Area $\%$ & & Retention Time & Area & Area $\%$ \\
\hline 1 & 13.012 & 35217609 & 97.485 & 1 & 13.589 & 497322 & 6.831 \\
\hline 2 & 15.738 & 908662 & 2.515 & 2 & 16.519 & 6782666 & 93.169 \\
\hline Total & & 36126271 & 100 & Total & & 7279988 & 100 \\
\hline
\end{tabular}
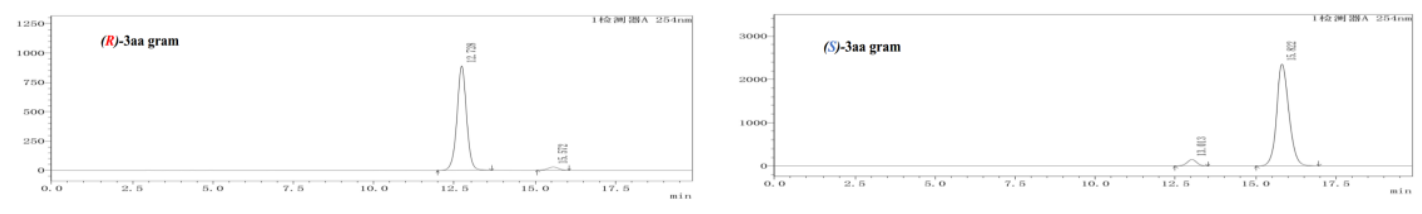

\begin{tabular}{|c|c|c|c|c|c|c|c|}
\hline & Retention Time & Area & Area $\%$ & & Retention Time & Area & Area $\%$ \\
\hline 1 & 12.728 & 18380361 & 96.608 & 1 & 13.013 & 3147273 & 4.787 \\
\hline 2 & 15.572 & 645415 & 3.392 & 2 & 15.822 & 62595986 & 95.213 \\
\hline Total & & 19025776 & 100 & Total & & 65743259 & 100 \\
\hline
\end{tabular}




\section{1-benzyl-3-hydroxy-5,7-dimethyl-3-(phenylethynyl) indolin-2-one (3ba):}

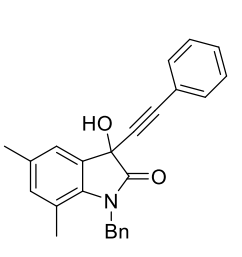

${ }^{1} \mathrm{H} \mathrm{NMR}\left(600 \mathrm{MHz}, \mathrm{CDCl}_{3}\right) \delta 7.45(\mathrm{~d}, J=7.5 \mathrm{~Hz}, 2 \mathrm{H}), 7.28-7.33(\mathrm{~m}$, $7 \mathrm{H}), 7.17(\mathrm{~d}, J=7.5 \mathrm{~Hz}, 2 \mathrm{H}), 6.81(\mathrm{~s}, 1 \mathrm{H}), 5.16(\mathrm{q}, J=16.8 \mathrm{~Hz}, 2 \mathrm{H})$, $3.95(\mathrm{~s}, 1 \mathrm{H}), 2.29(\mathrm{~s}, 3 \mathrm{H}), 2.19(\mathrm{~s}, 3 \mathrm{H}) .{ }^{13} \mathrm{C} \mathrm{NMR}\left(150 \mathrm{MHz}, \mathrm{CDCl}_{3}\right)$ $\delta 175.1,137.5,136.8,134.7,133.5,132.0,129.6,128.9,128.1,127.2$,

3ba $125.5,123.4,121.7,120.3,86.3,85.9,69.2,45.2,20.7,18.5$.

( $R$ )-3ba, white solid, $34.9 \mathrm{mg}, 95 \%$ yield (dichloromethane/ethyl acetate $=40: 1$ ), $91 \%$ $e e,[\alpha]_{\mathrm{D}}^{26}=+7.0\left(\mathrm{c}=0.71, \mathrm{CH}_{2} \mathrm{Cl}_{2}\right)$; HPLC: Daicel Chiralpak IA, n-hexane/2-propanol $=85 / 15$, flow rate $=1.0 \mathrm{~mL} / \mathrm{min}, \lambda=254 \mathrm{~nm}$, retention time: $12.3 \mathrm{~min}$ (major), 17.1 $\min$ (minor);

(S)-3ba, white solid, $36.3 \mathrm{mg}$, 99\% yield (dichloromethane/ethyl acetate $=40: 1$ ), 82\% ee. $[\alpha]_{\mathrm{D}}^{26}=-4.5\left(\mathrm{c}=0.95, \mathrm{CH}_{2} \mathrm{Cl}_{2}\right)$; HPLC: Daicel Chiralpak IA, n-hexane/2-propanol = $85 / 15$, flow rate $=1.0 \mathrm{~mL} / \mathrm{min}, \lambda=254 \mathrm{~nm}$, retention time: $12.1 \mathrm{~min}$ (minor), $16.5 \mathrm{~min}$ (major).
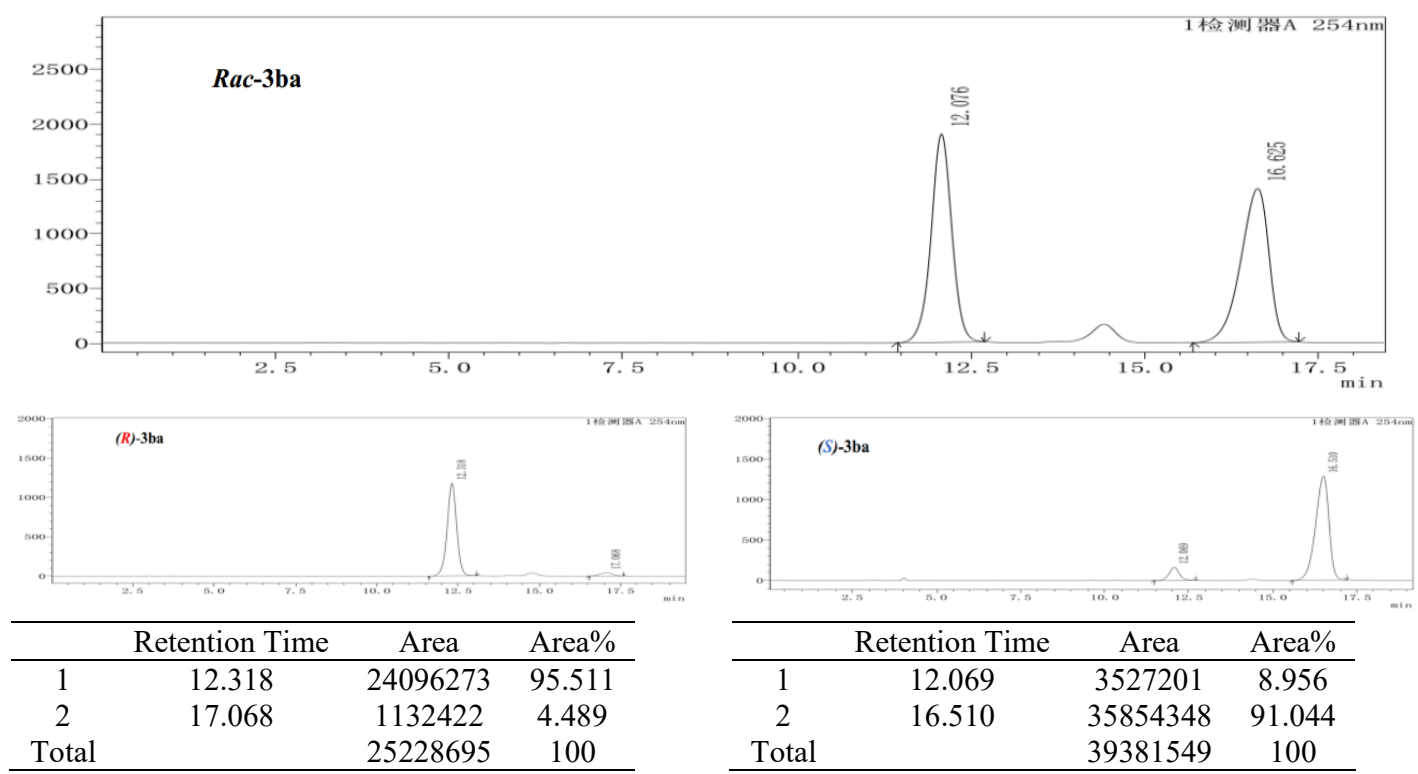


\section{1-benzyl-5-bromo-3-hydroxy-3-(phenylethynyl) indolin-2-one (3ca):}

${ }^{1} \mathrm{H} \mathrm{NMR}\left(600 \mathrm{MHz}, \mathrm{CDCl}_{3}\right) \delta 7.73(\mathrm{~s}, 1 \mathrm{H}), 7.45(\mathrm{~d}, J=7.7 \mathrm{~Hz}, 2 \mathrm{H})$, 7.35-7.24 (m, 9H), $6.57(\mathrm{~d}, J=8.4 \mathrm{~Hz}, 1 \mathrm{H}), 4.90(\mathrm{~s}, 2 \mathrm{H}), 4.28(\mathrm{~d}, J=$ $29.2 \mathrm{~Hz} 1 \mathrm{H}) .{ }^{1} \mathrm{H}$ NMR (600 MHz, DMSO-d6) $\delta 7.68(\mathrm{~d}, J=2.0 \mathrm{~Hz}$, $1 \mathrm{H}), 7.54-7.49(\mathrm{~m}, 2 \mathrm{H}), 7.48-7.25(\mathrm{~m}, 10 \mathrm{H}), 6.95(\mathrm{~d}, J=8.4 \mathrm{~Hz}, 1 \mathrm{H})$, $4.94(\mathrm{q}, J=15.9 \mathrm{~Hz}, 2 \mathrm{H}) .{ }^{13} \mathrm{C} \mathrm{NMR}\left(150 \mathrm{MHz}, \mathrm{CDCl}_{3}\right) \delta 172.7,140.0$, 133.4, 132.1, 131.1, 129.7, 128.1, 127.9,127.2, 127.0, 126.9, 126.0, 120.2, 115.4, 110.4, $86.1,83.7,68.4,43.1$.

(R)-3ca, white solid, $40.9 \mathrm{mg}, 98 \%$ yield (dichloromethane/ethyl acetate $=50: 1$ ), 93\% $e e,[\alpha]_{\mathrm{D}}^{26}=-129.3\left(\mathrm{c}=1.40, \mathrm{CH}_{2} \mathrm{Cl}_{2}\right)$; HPLC: Daicel Chiralpak IA, n-hexane/2-propanol $=97 / 3$, flow rate $=1.0 \mathrm{~mL} / \mathrm{min}, \lambda=254 \mathrm{~nm}$, retention time: $65.3 \mathrm{~min}$ (major), $72.9 \mathrm{~min}$ (minor);

(S)-3ca, white solid, $41.3 \mathrm{mg}, 99 \%$ yield (dichloromethane/ethyl acetate $=50: 1$ ), 82\% $e e,[\alpha]_{\mathrm{D}}^{26}=+102\left(\mathrm{c}=1.53, \mathrm{CH}_{2} \mathrm{Cl}_{2}\right)\left(\operatorname{ref} .1:(S), 92 \% e e,[\alpha]_{\mathrm{D}}^{20}=+135.1(\mathrm{c}=0.51\right.$ in $\left.\mathrm{CH}_{2} \mathrm{Cl}_{2}\right)$ ); HPLC: Daicel Chiralpak IA, n-hexane/2-propanol $=97 / 3$, flow rate $=1.0$ $\mathrm{mL} / \mathrm{min}, \lambda=254 \mathrm{~nm}$, retention time: $71.5 \mathrm{~min}$ (minor), $77.9 \mathrm{~min}$ (major).
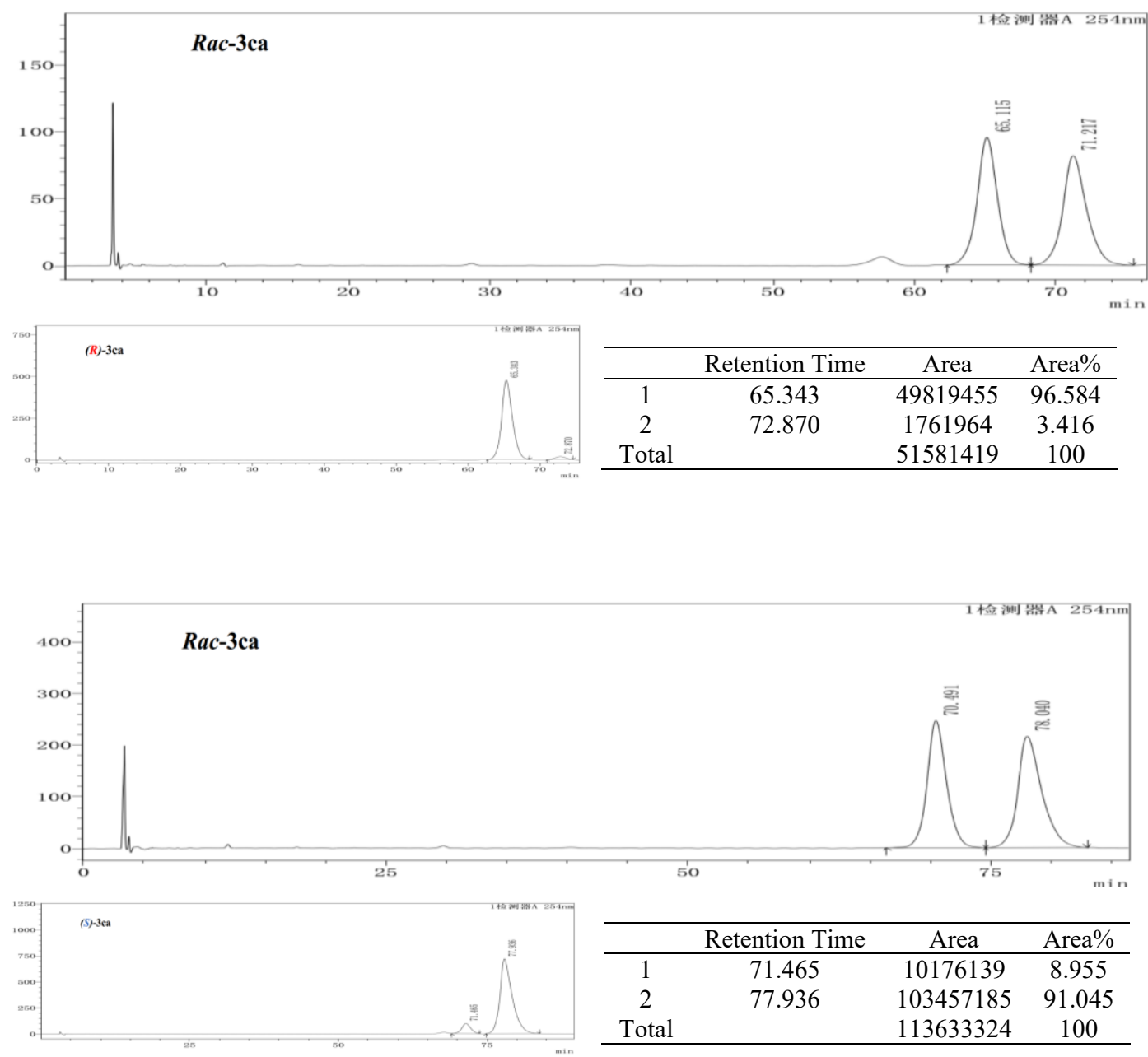


\section{1-benzyl-3-hydroxy-5-methyl-3-(phenylethynyl) indolin-2-one (3da):}

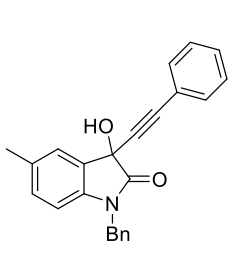

${ }^{1} \mathrm{H}$ NMR (600 MHz, $\left.\mathrm{CDCl}_{3}\right) \delta$ 7.48-7.41 (m, 3H), 7.34-7.25 (m, 8H), 7.04-7.02 (m, 1H), 6.61-6.59 (m, 1H), $4.90(\mathrm{~s}, 2 \mathrm{H}), 3.76(\mathrm{~s}, 1 \mathrm{H}), 2.32$ $(\mathrm{s}, 3 \mathrm{H}) .{ }^{13} \mathrm{C} \mathrm{NMR}\left(150 \mathrm{MHz}, \mathrm{CDCl}_{3}\right) \delta 174.2,139.6,135.0,133.5$, 132.0, 130.6, 128.9,128.8, 128.2, 127.7, 127.1, 125.5, 121.6, 109.7,

3da

86.4, 85.6, 69.7, 44.1, 21.0. HRMS (ESI) $m / z:[\mathrm{M}+\mathrm{H}]^{+}$Calcd for $\mathrm{C}_{24} \mathrm{H}_{20} \mathrm{NO}_{2}{ }^{+}$354.1489; Found: 354.1478.

( $R$ )-3da, white solid, $31.8 \mathrm{mg}, 90 \%$ yield (dichloromethane/ethyl acetate $=40: 1$ ), 93\% $e e,[\alpha]_{\mathrm{D}}^{26}=-52\left(\mathrm{c}=1.01, \mathrm{CH}_{2} \mathrm{Cl}_{2}\right)$; HPLC: Daicel Chiralpak IA, n-hexane/2-propanol = $85 / 15$, flow rate $=1.0 \mathrm{~mL} / \mathrm{min}, \lambda=254 \mathrm{~nm}$, retention time: $13.3 \mathrm{~min}$ (major), $15.9 \mathrm{~min}$ (minor);

(S)-3da, white solid, $32.1 \mathrm{mg}, 91 \%$ yield (dichloromethane/ethyl acetate $=40: 1$ ), 88\% $e e,[\alpha]_{\mathrm{D}}^{26}=+50.8\left(\mathrm{c}=0.61, \mathrm{CH}_{2} \mathrm{Cl}_{2}\right)$; HPLC: Daicel Chiralpak IA, n-hexane/2-propanol $=85 / 15$, flow rate $=1.0 \mathrm{~mL} / \mathrm{min}, \lambda=254 \mathrm{~nm}$, retention time: $12.4 \mathrm{~min}$ (minor), 14.5 $\min$ (major).
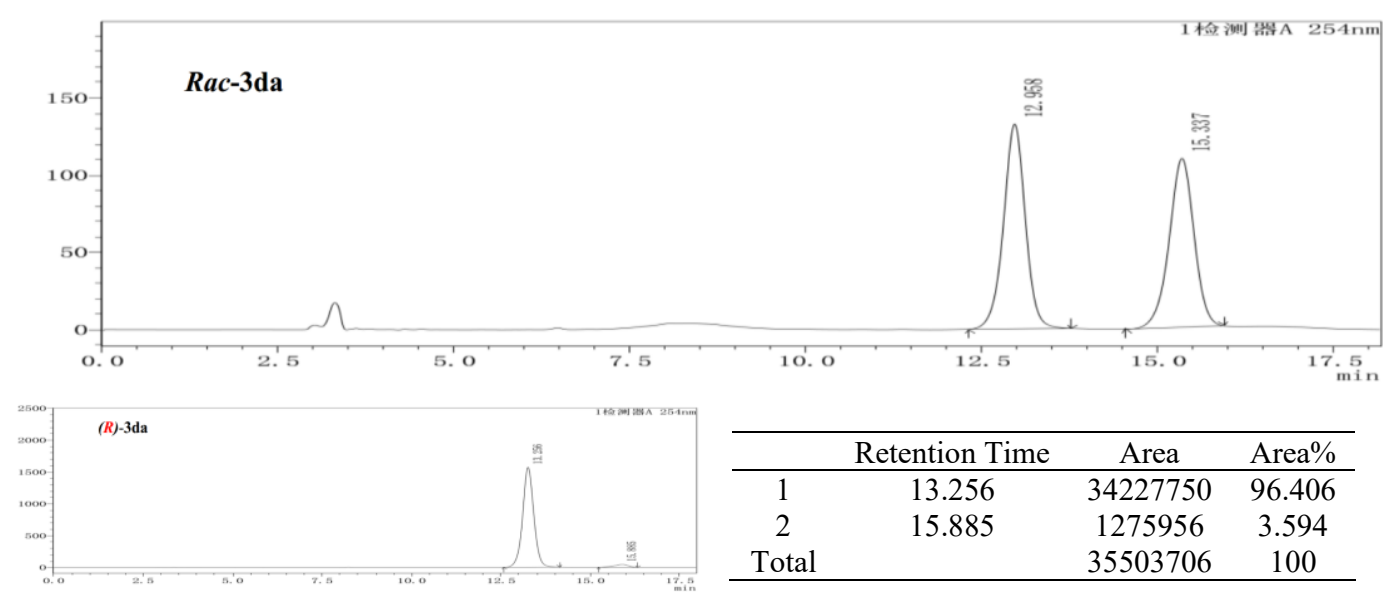

\begin{tabular}{cccc}
\hline & Retention Time & Area & Area\% \\
\hline 1 & 13.256 & 34227750 & 96.406 \\
2 & 15.885 & 1275956 & 3.594 \\
Total & & 35503706 & 100 \\
\hline
\end{tabular}

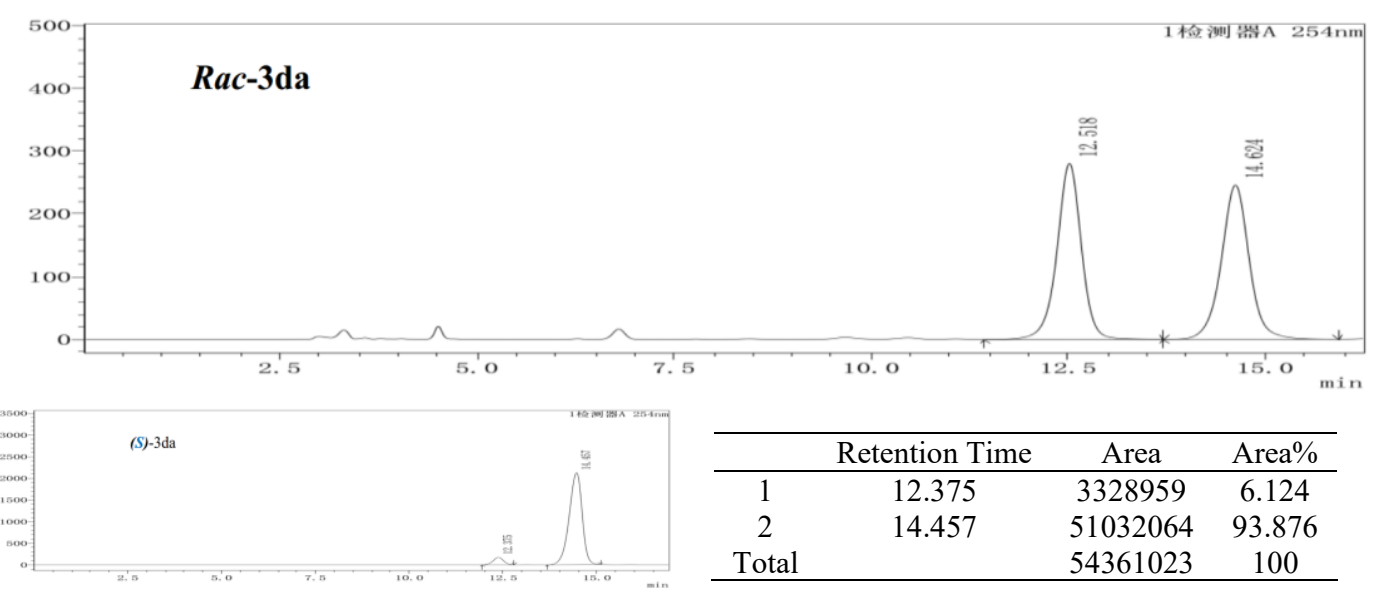




\section{1-benzyl-5-chloro-3-hydroxy-3-(phenylethynyl) indolin-2-one (3ea):}

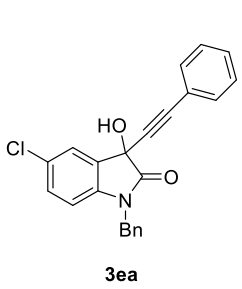

${ }^{1} \mathrm{H}$ NMR $\left(600 \mathrm{MHz}, \mathrm{CDCl}_{3}\right) \delta$ 7.61-7.59 (m, 1H), 7.48-7.42 (m, 2H), 7.35-7.25 (m, 8H), $7.19(\mathrm{dd}, J=8.4,2.1 \mathrm{~Hz}, 1 \mathrm{H}), 6.64-6.59(\mathrm{~m}, 1 \mathrm{H})$, $4.90(\mathrm{~s}, 2 \mathrm{H}), 4.30(\mathrm{~s}, 1 \mathrm{H}) .{ }^{13} \mathrm{C} \mathrm{NMR}\left(150 \mathrm{MHz}, \mathrm{CDCl}_{3}\right) \delta 173.8,140.5$, $134.5,132.1,130.4,130.2,129.2,129.1,128.9,128.2,127.9,127.1$, $125.3,121.3,110.9,87.0,84.7,69.5,44.2$.

(R)-3ea, white solid, $36.6 \mathrm{mg}, 98 \%$ yield (dichloromethane/ethyl acetate $=50: 1$ ), 94\% $e e,[\alpha]_{\mathrm{D}}^{26}=-73.6\left(\mathrm{c}=0.63, \mathrm{CH}_{2} \mathrm{Cl}_{2}\right)$; HPLC: Daicel Chiralpak IA, n-hexane/2-propanol $=95 / 5$, flow rate $=1.0 \mathrm{~mL} / \mathrm{min}, \lambda=254 \mathrm{~nm}$, retention time: $37.5 \mathrm{~min}$ (major), $41.6 \mathrm{~min}$ (minor);

(S)-3ea, white solid, $37.0 \mathrm{mg}, 99 \%$ yield (dichloromethane/ethyl acetate $=50: 1$ ), 76\% $e e,[\alpha]_{\mathrm{D}}^{26}=+61\left(\mathrm{c}=1.51, \mathrm{CH}_{2} \mathrm{Cl}_{2}\right)$; HPLC: Daicel Chiralpak IA, n-hexane/2-propanol = $95 / 5$, flow rate $=1.0 \mathrm{~mL} / \mathrm{min}, \lambda=254 \mathrm{~nm}$, retention time: $40.8 \mathrm{~min}$ (minor), $44.6 \mathrm{~min}$ (major).
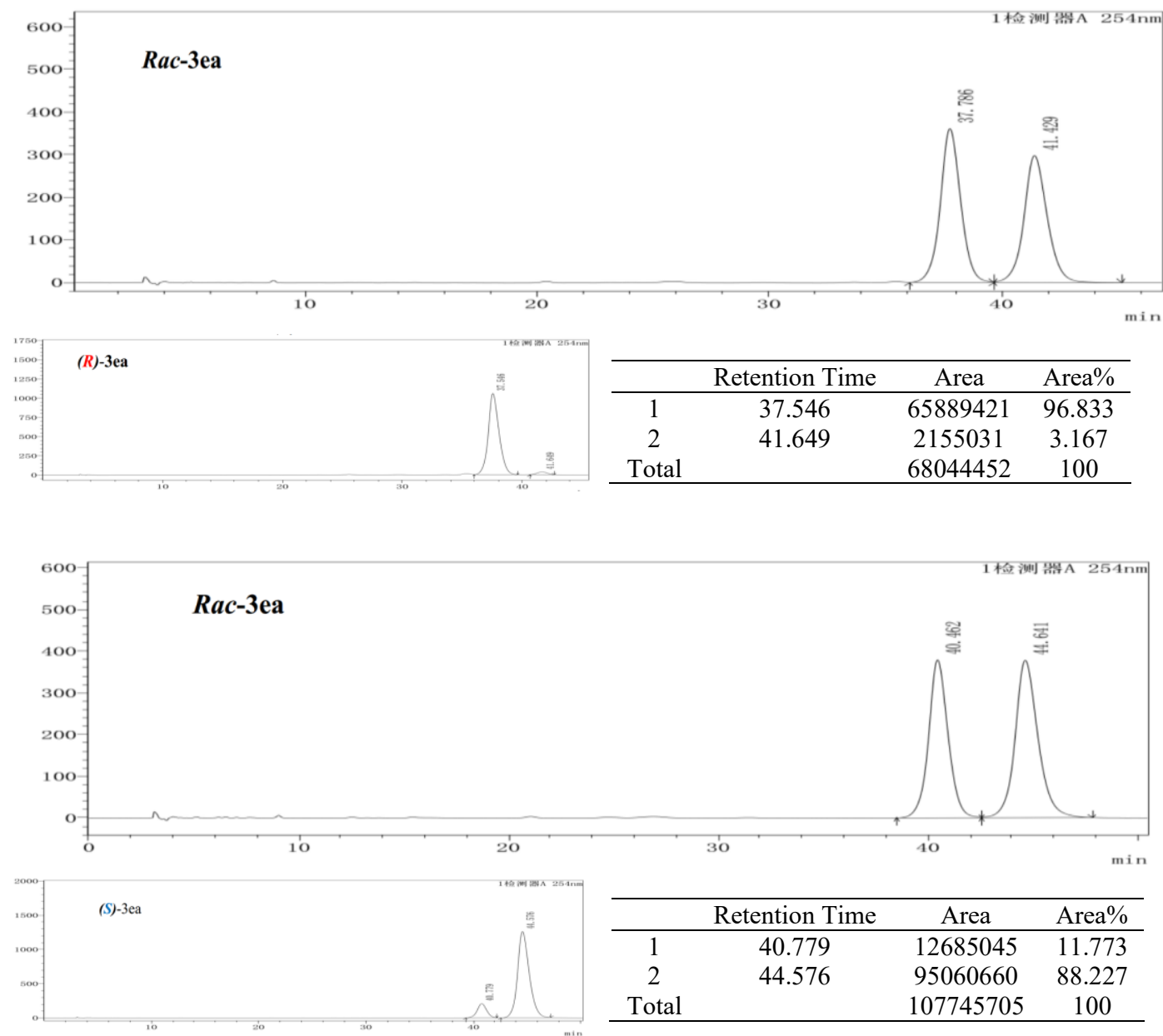


\section{1-benzyl-5-fluoro-3-hydroxy-3-(phenylethynyl) indolin-2-one (3fa):}

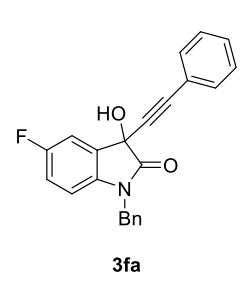

${ }^{1} \mathrm{H}$ NMR $\left(600 \mathrm{MHz}, \mathrm{CDCl}_{3}\right) \delta 7.43(\mathrm{~d}, J=7.4 \mathrm{~Hz}, 2 \mathrm{H}), 7.39-7.36(\mathrm{~m}$, $1 \mathrm{H}), 7.32-7.23(\mathrm{~m}, 8 \mathrm{H}), 6.94-6.90(\mathrm{~m}, 1 \mathrm{H}), 6.63-6.59(\mathrm{~m}, 1 \mathrm{H}), 4.93-$ $4.87(\mathrm{~m}, 2 \mathrm{H}), 4.44(\mathrm{~s}, 1 \mathrm{H}) .{ }^{13} \mathrm{C} \mathrm{NMR}\left(150 \mathrm{MHz}, \mathrm{CDCl}_{3}\right) \delta 174.1,160.5$, 158.9, 137.9 (d, $J=2.1 \mathrm{~Hz}), 134.6,132.1,129.1,128.9,128.2,127.9$, 127.1, 121.3, $116.6(\mathrm{~d}, J=23.7 \mathrm{~Hz}), 112.9(\mathrm{~d}, J=25.2 \mathrm{~Hz}), 110.6(\mathrm{~d}$, $J=7.9 \mathrm{~Hz}), 86.9,84.9,69.7,44.2$.

$(R)$-3fa, white solid, $35.3 \mathrm{mg}, 99 \%$ yield (dichloromethane/ethyl acetate $=50: 1$ ), 91\% $e e,[\alpha]_{\mathrm{D}}^{26}=-10.6\left(\mathrm{c}=0.75, \mathrm{CH}_{2} \mathrm{Cl}_{2}\right)$; HPLC: Daicel Chiralpak IA, n-hexane/2-propanol $=85 / 15$, flow rate $=1.0 \mathrm{~mL} / \mathrm{min}, \lambda=254 \mathrm{~nm}$, retention time: $13.1 \mathrm{~min}$ (major), 15.8 $\min$ (minor);

(S)-3fa, white solid, $35.5 \mathrm{mg}, 99 \%$ yield (dichloromethane/ethyl acetate $=50: 1$ ), 82\% $e e,[\alpha]_{\mathrm{D}}^{26}=+9.1\left(\mathrm{c}=0.33, \mathrm{CH}_{2} \mathrm{Cl}_{2}\right)\left(\operatorname{ref} .1:(S), 83 \% e e,[\alpha]_{\mathrm{D}}^{20}=+10.3(\mathrm{c}=0.54\right.$ in $\mathrm{CH}_{2} \mathrm{Cl}_{2}$ )); HPLC: Daicel Chiralpak IA, n-hexane/2-propanol $=85 / 15$, flow rate $=1.0$ $\mathrm{mL} / \mathrm{min}, \lambda=254 \mathrm{~nm}$, retention time: $12.1 \mathrm{~min}$ (minor), $16.5 \mathrm{~min}$ (major).
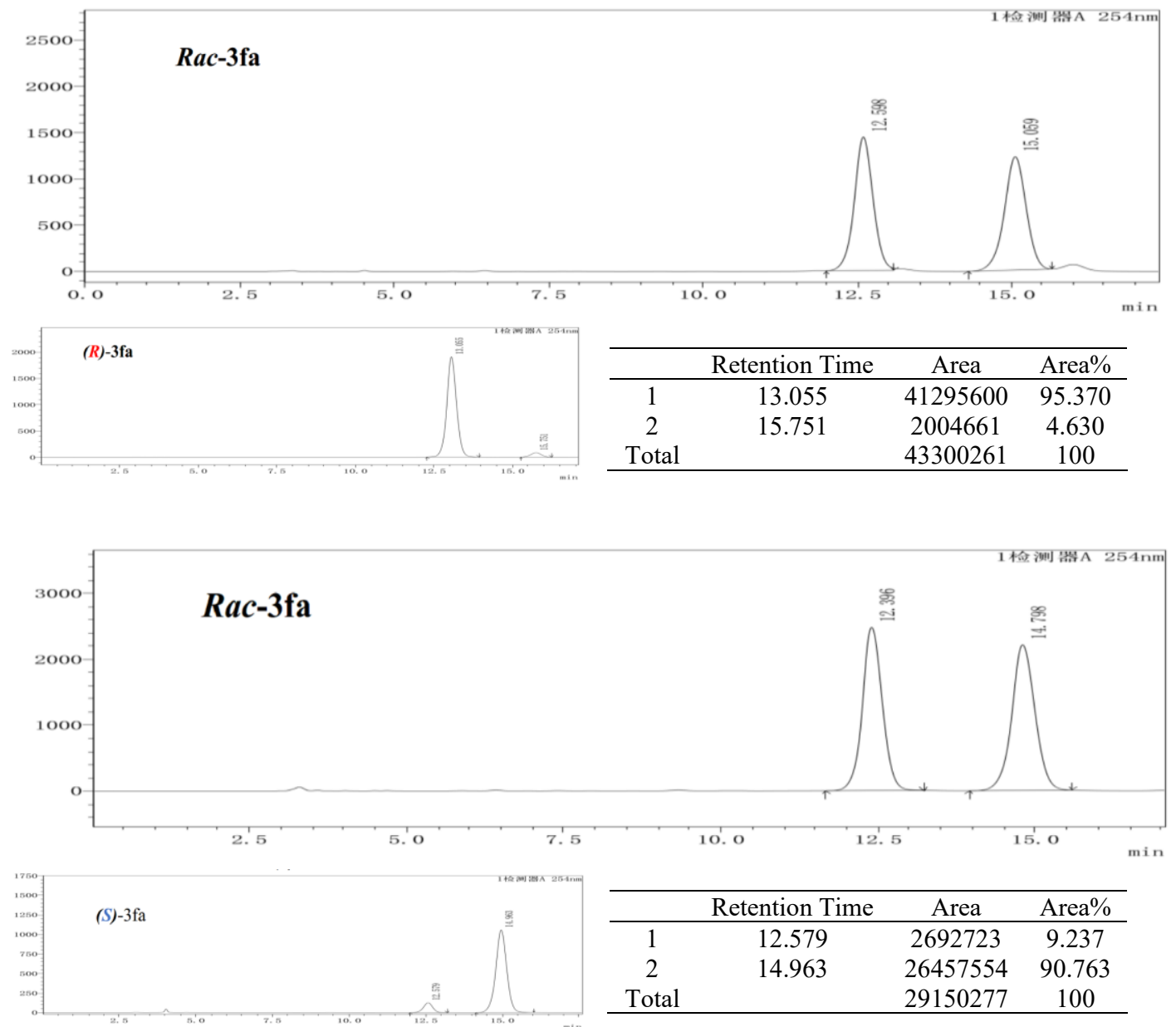

\begin{tabular}{cccc}
\hline & Retention Time & Area & Area\% \\
\hline 1 & 12.579 & 2692723 & 9.237 \\
2 & 14.963 & 26457554 & 90.763 \\
Total & & 29150277 & 100 \\
\hline
\end{tabular}




\section{1-benzyl-3-hydroxy-5-methoxy-3-(phenylethynyl) indolin-2-one (3ga):}

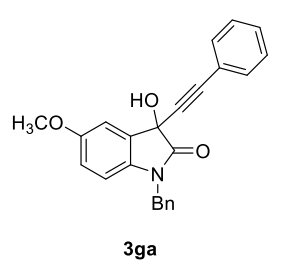

${ }^{1} \mathrm{H}$ NMR $\left(600 \mathrm{MHz}, \mathrm{CDCl}_{3}\right) \delta$ 7.48-7.42 (m, 2H), 7.32-7.24 (m, 9H), $6.76(\mathrm{dd}, J=8.6,2.5 \mathrm{~Hz}, 1 \mathrm{H}), 6.61-6.59(\mathrm{~m}, 1 \mathrm{H}), 4.92-4.86(\mathrm{~m}, 2 \mathrm{H})$, $4.17(\mathrm{~s}, 1 \mathrm{H}), 3.77(\mathrm{~s}, 3 \mathrm{H}) .{ }^{13} \mathrm{C} \mathrm{NMR}\left(150 \mathrm{MHz}, \mathrm{CDCl}_{3}\right) \delta 174.0$, $156.7,135.3,135.0,132.0,130.0,128.9,128.8,128.2,127.7,127.1$, $121.6,115.3,111.3,110.5,86.6,85.5,69.9,55.8,44.1$.

(R)-3ga, white solid, $30.6 \mathrm{mg}, 83 \%$ yield (dichloromethane/ethyl acetate $=30: 1$ ), $90 \%$ $e e,[\alpha]_{\mathrm{D}}^{26}=-100.5\left(\mathrm{c}=0.61, \mathrm{CH}_{2} \mathrm{Cl}_{2}\right)$; HPLC: Daicel Chiralpak IA, n-hexane/2-propanol $=85 / 15$, flow rate $=1.0 \mathrm{~mL} / \mathrm{min}, \lambda=254 \mathrm{~nm}$, retention time: $16.5 \mathrm{~min}$ (major), 21.1 $\min ($ minor);

(S)-3ga, white solid, $29.5 \mathrm{mg}, 80 \%$ yield (dichloromethane/ethyl acetate $=30: 1$ ), $77 \%$ $e e,[\alpha]_{\mathrm{D}}^{26}=+72.2\left(\mathrm{c}=0.39, \mathrm{CH}_{2} \mathrm{Cl}_{2}\right)\left(\operatorname{ref} .1:(S), 89 \% e e,[\alpha]_{\mathrm{D}}^{20}=+104.6(\mathrm{c}=0.54\right.$ in $\mathrm{CH}_{2} \mathrm{Cl}_{2}$ )); HPLC: Daicel Chiralpak IA, n-hexane/2-propanol $=85 / 15$, flow rate $=1.0$ $\mathrm{mL} / \mathrm{min}, \lambda=254 \mathrm{~nm}$, retention time: $16.3 \mathrm{~min}$ (minor), $20.9 \mathrm{~min}$ (major).
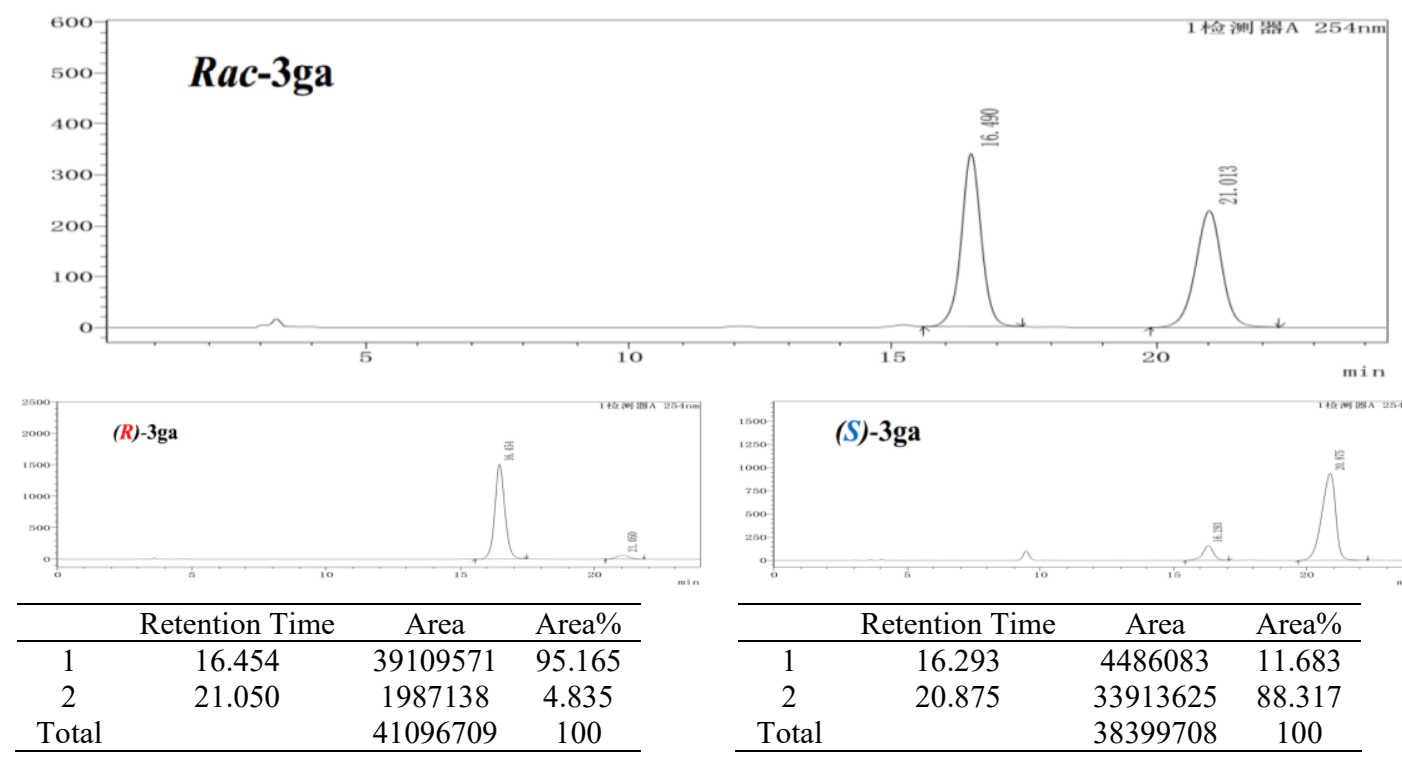
1-benzyl-6-bromo-3-hydroxy-3-(phenylethynyl) indolin-2-one (3ha):

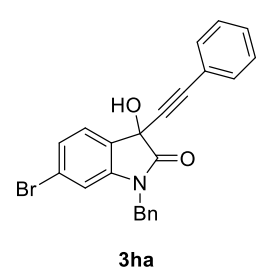

${ }^{1} \mathrm{H} \mathrm{NMR}\left(600 \mathrm{MHz}, \mathrm{CDCl}_{3}\right) \delta$ 7.48-7.46 (m, $\left.1 \mathrm{H}\right), 7.42(\mathrm{~d}, J=7.1 \mathrm{~Hz}$, 2H), 7.32-7.25 (m, 9H), 6.86-6.84 (m, 1H), 4.91-4.84 (m, 2H), 4.21 (s, 1H). ${ }^{13} \mathrm{C}$ NMR $\left(150 \mathrm{MHz}, \mathrm{CDCl}_{3}\right) \delta 174.0,143.3,134.4,132.0$, 129.1, 129.0, 128.2, 128.0, 127.9, 127.0, 126.6, 126.0, 124.0, 121.4,

$113.2,86.7,84.9,69.2,67.9,44.1,25.5$. HRMS (ESI) $\mathrm{m} / z:[\mathrm{M}+\mathrm{H}]^{+}$ Calcd for $\mathrm{C}_{23} \mathrm{H}_{17} \mathrm{BrNO}_{2}{ }^{+}$418.0437; Found: 418.0436.

$(R)$-3ha, white solid, $37.9 \mathrm{mg}, 91 \%$ yield (dichloromethane/ethyl acetate $=50: 1$ ), 93\% $e e,[\alpha]_{\mathrm{D}}^{26}=+3.9\left(\mathrm{c}=1.54, \mathrm{CH}_{2} \mathrm{Cl}_{2}\right)$; HPLC: Daicel Chiralpak IA, n-hexane/2-propanol $=85 / 15$, flow rate $=1.0 \mathrm{~mL} / \mathrm{min}, \lambda=254 \mathrm{~nm}$, retention time: $14.4 \mathrm{~min}$ (major), 16.4 $\min$ (minor);

(S)-3ha, white solid, $36.3 \mathrm{mg}, 87 \%$ yield (dichloromethane/ethyl acetate $=50: 1$ ), 88\% $e e .[\alpha]_{\mathrm{D}}^{26}=-2.9\left(\mathrm{c}=0.35, \mathrm{CH}_{2} \mathrm{Cl}_{2}\right)$; HPLC: Daicel Chiralpak IA, n-hexane/2-propanol = $85 / 15$, flow rate $=1.0 \mathrm{~mL} / \mathrm{min}, \lambda=254 \mathrm{~nm}$, retention time: $14.3 \mathrm{~min}$ (minor), $16.2 \mathrm{~min}$ (major).

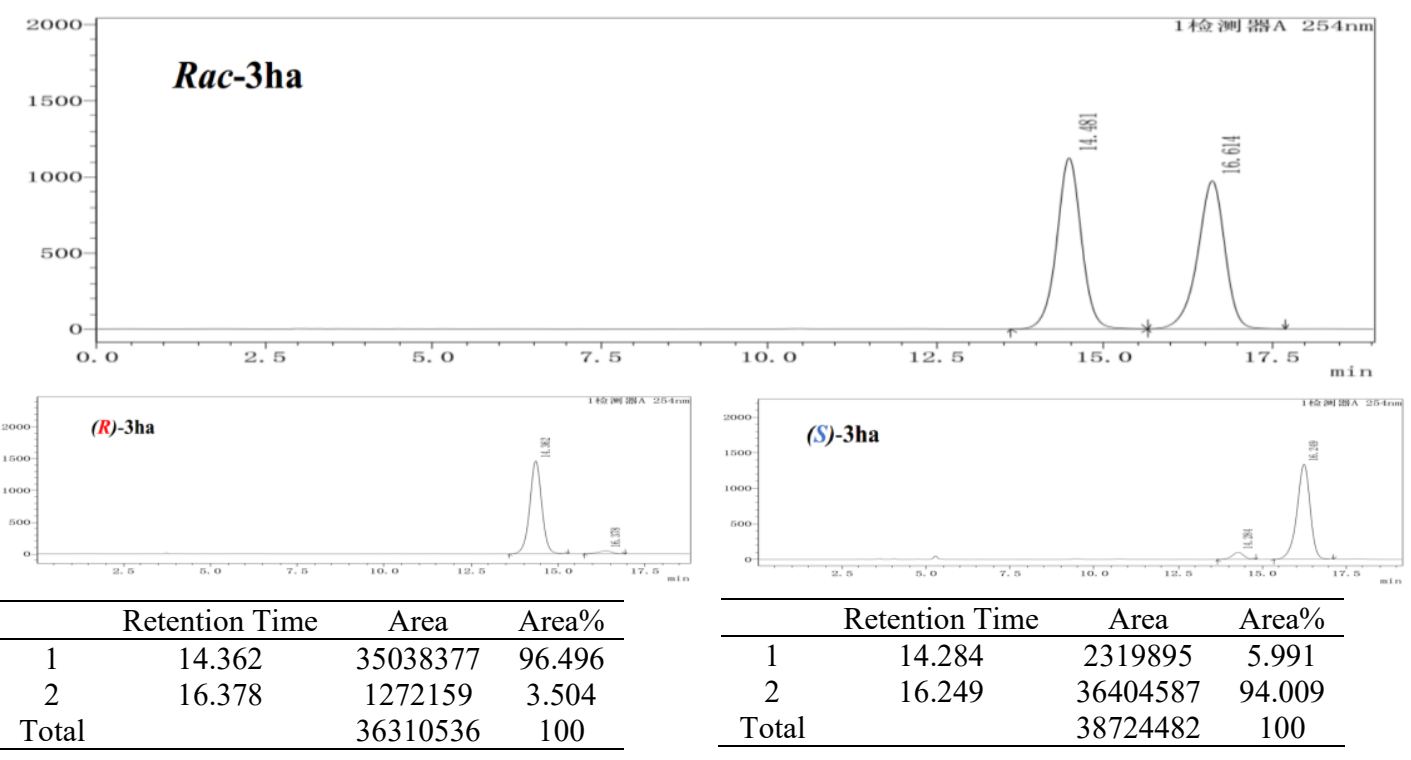




\section{1-benzyl-6-chloro-3-hydroxy-3-(phenylethynyl) indolin-2-one (3ia):}

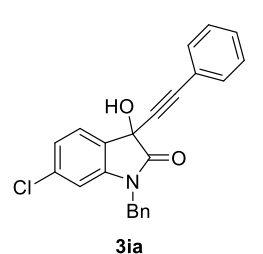

${ }^{1} \mathrm{H} \mathrm{NMR}\left(600 \mathrm{MHz}, \mathrm{CDCl}_{3}\right) \delta 7.52(\mathrm{~d}, J=8.0 \mathrm{~Hz}, 1 \mathrm{H}), 7.43-7.42(\mathrm{~m}$, $2 \mathrm{H}), 7.35-7.25(\mathrm{~m}, 8 \mathrm{H}), 7.08(\mathrm{dd}, J=8.0,1.8 \mathrm{~Hz}, 1 \mathrm{H}), 6.71(\mathrm{~d}, J=$ $1.8 \mathrm{~Hz}, 1 \mathrm{H}), 4.94-4.83(\mathrm{~m}, 2 \mathrm{H}), 4.00(\mathrm{~s}, 1 \mathrm{H}) .{ }^{13} \mathrm{C} \mathrm{NMR}(150 \mathrm{MHz}$, $\left.\mathrm{CDCl}_{3}\right) \delta 174.2,143.2,136.1,134.4,132.0,129.1,129.0,128.2$, 128.0, 127.3, 127.1, 125.7, 123.7, 121.3, 110.5, 86.8, 84.9, 69.2, 44.2.

(R)-3ia, white solid, $37.1 \mathrm{mg}, 99 \%$ yield (dichloromethane/ethyl acetate $=30: 1$ ), 94\% $e e,[\alpha]_{\mathrm{D}}^{26}=-1.8\left(\mathrm{c}=1.1, \mathrm{CH}_{2} \mathrm{Cl}_{2}\right)$; HPLC: Daicel Chiralpak IA, n-hexane/2-propanol = $85 / 15$, flow rate $=1.0 \mathrm{~mL} / \mathrm{min}, \lambda=254 \mathrm{~nm}$, retention time: $13.6 \mathrm{~min}$ (major), $15.4 \mathrm{~min}$ (minor);

(S)-3ia, white solid, $31.0 \mathrm{mg}, 83 \%$ yield (dichloromethane/ethyl acetate $=30: 1$ ), $91 \%$ $e e,[\alpha]_{\mathrm{D}}^{26}=+1.5\left(\mathrm{c}=0.65, \mathrm{CH}_{2} \mathrm{Cl}_{2}\right)\left(\operatorname{ref} .1:(S), 87 \% e e,[\alpha]_{\mathrm{D}}^{20}=+2.0\left(\mathrm{c}=0.52\right.\right.$ in $\left.\left.\mathrm{CH}_{2} \mathrm{Cl}_{2}\right)\right)$; HPLC: Daicel Chiralpak IA, n-hexane/2-propanol $=85 / 15$, flow rate $=1.0 \mathrm{~mL} / \mathrm{min}, \lambda$ $=254 \mathrm{~nm}$, retention time: $13.8 \mathrm{~min}$ (minor), $15.6 \mathrm{~min}$ (major).

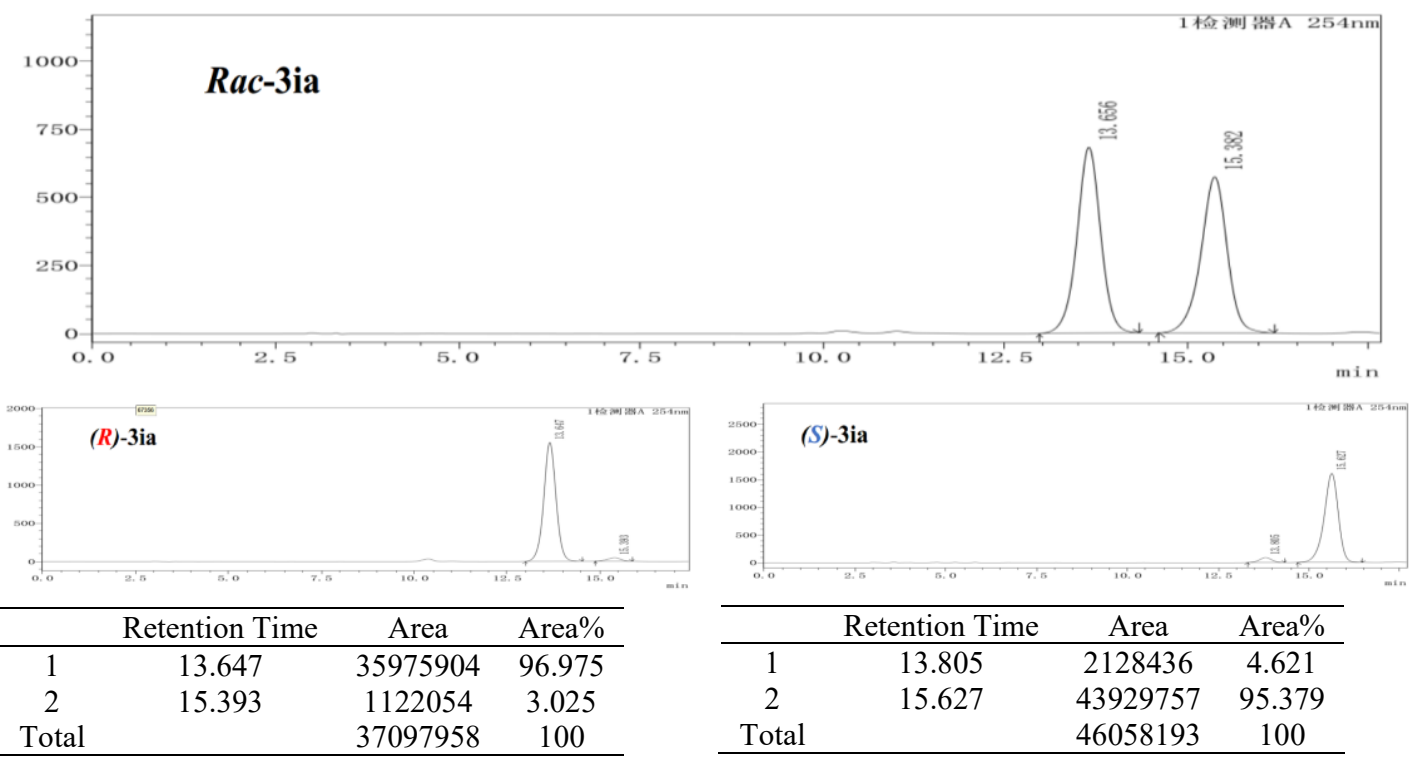




\section{1-benzyl-3-hydroxy-6-methoxy-3-(phenylethynyl) indolin-2-one (3ja):}

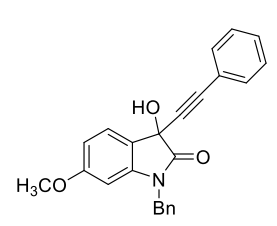

3ja

${ }^{1} \mathrm{H} \mathrm{NMR}\left(600 \mathrm{MHz}, \mathrm{CDCl}_{3}\right) \delta$ 7.53-7.51 (m, 1H), 7.46-7.42 (m, 2H), 7.32-7.24 (m, 8H), $6.58(\mathrm{dd}, J=8.3,2.2 \mathrm{~Hz}, 1 \mathrm{H}), 6.30-6.28(\mathrm{~m}, 1 \mathrm{H})$, $4.88(\mathrm{~s}, 2 \mathrm{H}), 3.87(\mathrm{~s}, 1 \mathrm{H}), 3.72(\mathrm{~s}, 3 \mathrm{H}) .{ }^{13} \mathrm{C} \mathrm{NMR}\left(150 \mathrm{MHz}, \mathrm{CDCl}_{3}\right)$ $\delta$ 174.6, 161.6, 143.5, 135.0, 132.0, 128.9, 128.8, 128.2, 127.8, 127.1, 125.7, 121.7, 121.0, 107.2, 98.0, 86.2, 85.7, 69.3, 55.5, 44.1. HRMS

(ESI) $m / z:[\mathrm{M}+\mathrm{H}]^{+}$Calcd for $\mathrm{C}_{24} \mathrm{H}_{20} \mathrm{NO}_{3}{ }^{+} 370.1438$; Found: 370.1427.

$(R)-3 \mathbf{j a}$, white solid, $26.9 \mathrm{mg}, 73 \%$ yield (dichloromethane/ethyl acetate $=50: 1$ ), 95\% $e e,[\alpha]_{\mathrm{D}}^{26}=-26.9\left(\mathrm{c}=0.26, \mathrm{CH}_{2} \mathrm{Cl}_{2}\right)$; HPLC Daicel Chiralpak IA, n-hexane/2-propanol $=90 / 10$, flow rate $=1.0 \mathrm{~mL} / \mathrm{min}, \lambda=254 \mathrm{~nm}$, retention time: $33.2 \mathrm{~min}$ (major), 36.2 $\min$ (minor);

(S)-3ja, white solid, $25.8 \mathrm{mg}, 70 \%$ yield (dichloromethane/ethyl acetate $=50: 1$ ), 97\% $e e,[\alpha]_{\mathrm{D}}^{26}=+15.6\left(\mathrm{c}=0.53, \mathrm{CH}_{2} \mathrm{Cl}_{2}\right)$; HPLC Daicel Chiralpak IA, n-hexane/2-propanol $=90 / 10$, flow rate $=1.0 \mathrm{~mL} / \mathrm{min}, \lambda=254 \mathrm{~nm}$, retention time: $32.4 \mathrm{~min}$ (minor), 35.0 $\min$ (major).
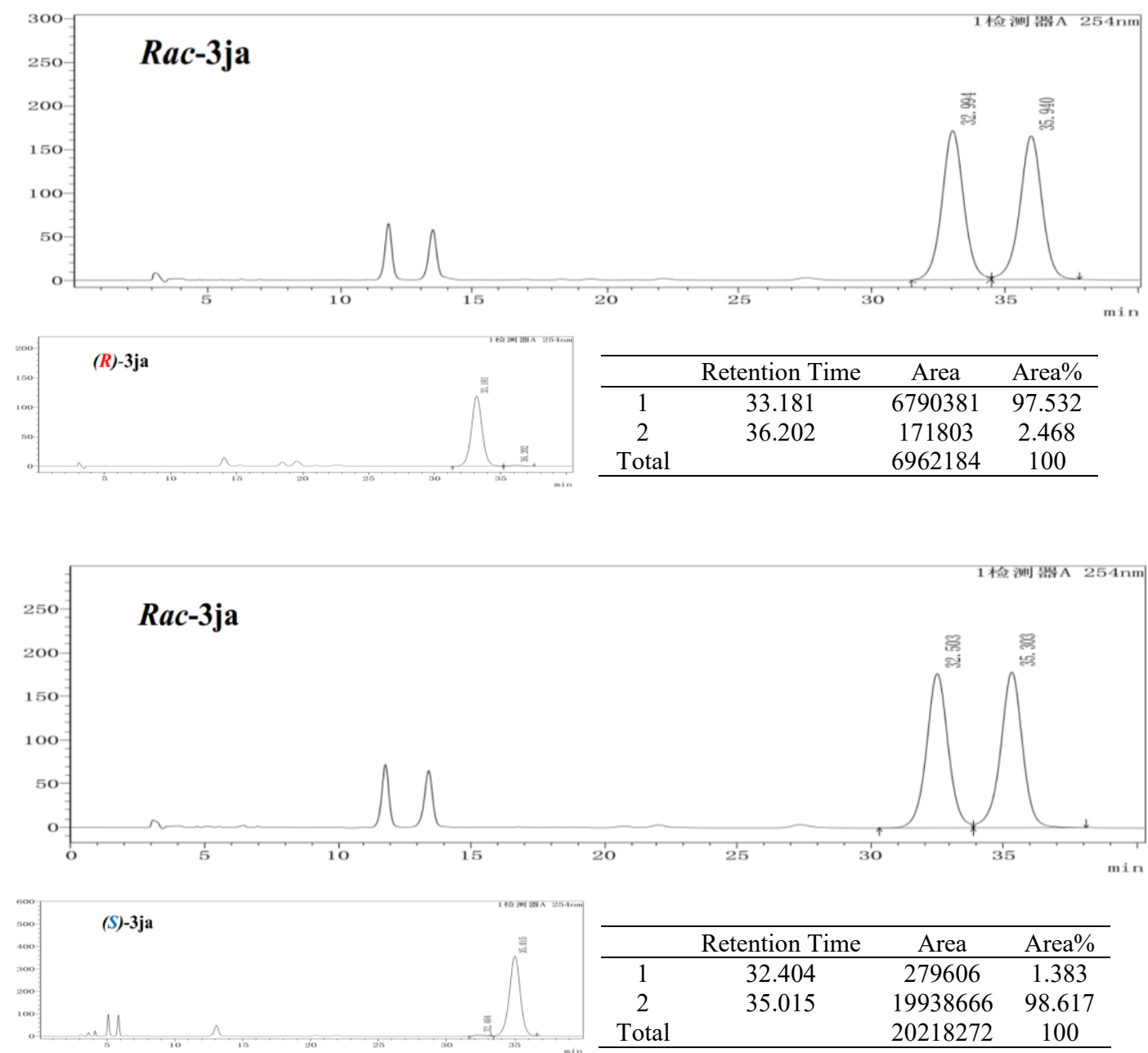
1-benzyl-7-bromo-3-hydroxy-3-(phenylethynyl) indolin-2-one (3ka):

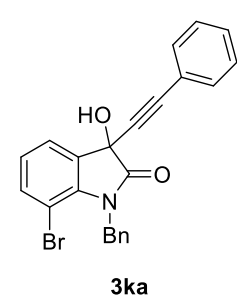

${ }^{1} \mathrm{H}$ NMR $\left(600 \mathrm{MHz}, \mathrm{CDCl}_{3}\right) \delta 7.60(\mathrm{dd}, J=7.4,1.2 \mathrm{~Hz}, 1 \mathrm{H}), 7.43-$ $7.40(\mathrm{~m}, 3 \mathrm{H}), 7.34-7.22(\mathrm{~m}, 8 \mathrm{H}), 7.03-6.97(\mathrm{~m}, 1 \mathrm{H}), 5.41(\mathrm{~s}, 2 \mathrm{H}), 3.95$ $(\mathrm{s}, 1 \mathrm{H}) .{ }^{13} \mathrm{C} \mathrm{NMR}\left(150 \mathrm{MHz}, \mathrm{CDCl}_{3}\right) \delta 174.0,138.6,135.5,135.1$, $131.0,128.1,127.9,127.6,127.2,126.2,125.1,124.1,123.1,120.3$, $102.0,85.9,84.0,67.9,43.8$.

$(R)$-3ka, white solid, $39.6 \mathrm{mg}, 95 \%$ yield (dichloromethane/ethyl acetate $=50: 1$ ), 92\% $e e,[\alpha]_{\mathrm{D}}^{26}=+92\left(\mathrm{c}=0.70, \mathrm{CH}_{2} \mathrm{Cl}_{2}\right)$; HPLC Daicel Chiralpak IA, n-hexane/2-propanol = $85 / 15$, flow rate $=1.0 \mathrm{~mL} / \mathrm{min}, \lambda=254 \mathrm{~nm}$, retention time: $10.8 \mathrm{~min}$ (major), $15.6 \mathrm{~min}$ (minor);

(S)-3ka, white solid, $37.5 \mathrm{mg}, 90 \%$ yield (dichloromethane/ethyl acetate $=50: 1$ ), 84\% $e e,[\alpha]_{\mathrm{D}}^{26}=-96.5\left(\mathrm{c}=0.86, \mathrm{CH}_{2} \mathrm{Cl}_{2}\right)\left(\operatorname{ref} .1:(S), 89 \% e e,[\alpha]_{\mathrm{D}}^{20}=-118.0(\mathrm{c}=0.54\right.$ in $\left.\mathrm{CH}_{2} \mathrm{Cl}_{2}\right)$ ); HPLC Daicel Chiralpak IA, n-hexane/2-propanol $=85 / 15$, flow rate $=1.0$ $\mathrm{mL} / \mathrm{min}, \lambda=254 \mathrm{~nm}$, retention time: $11.0 \mathrm{~min}$ (minor), $16.0 \mathrm{~min}$ (major).
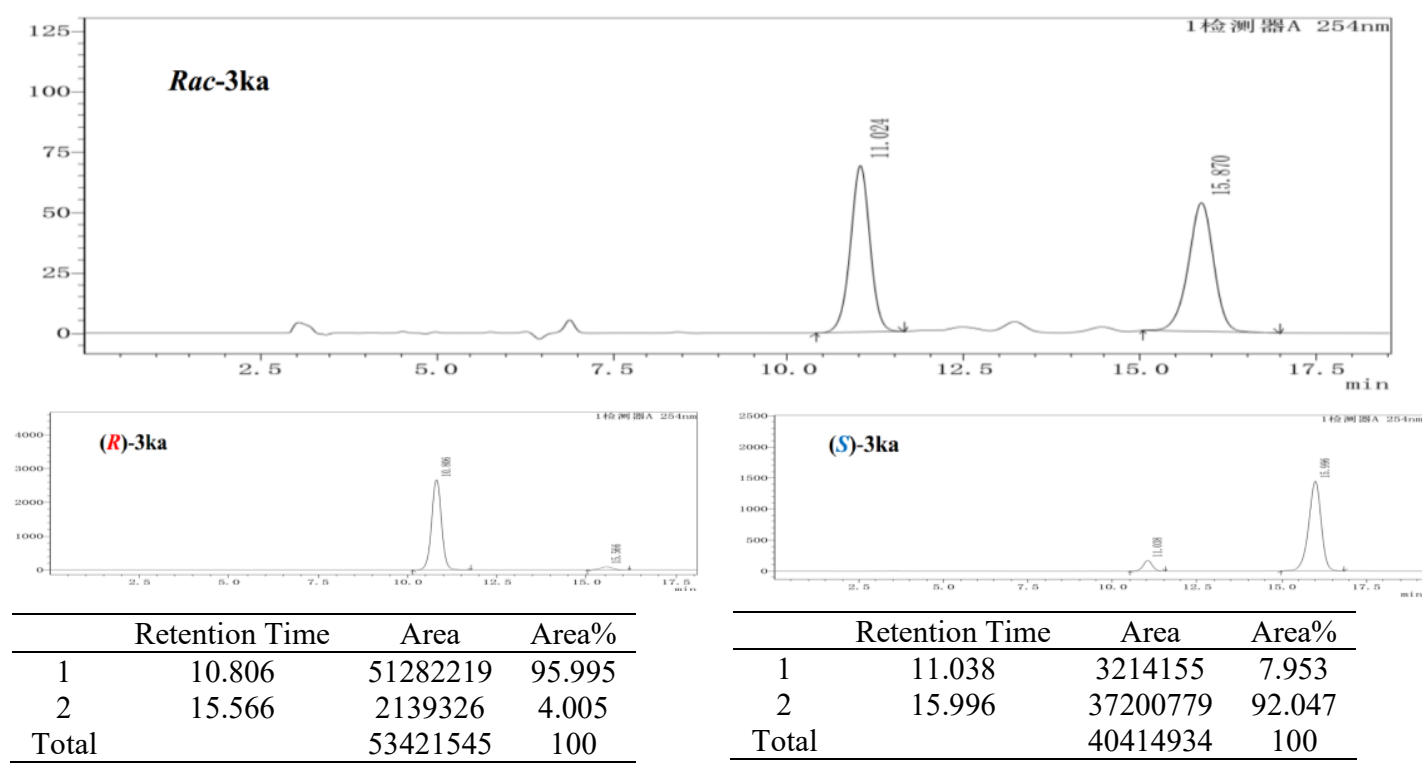


\section{1-benzyl-3-hydroxy-7-methyl-3-(phenylethynyl) indolin-2-one (3la):}

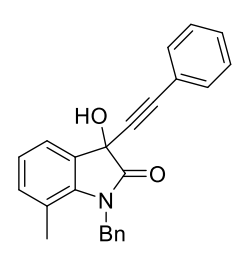

${ }^{1} \mathrm{H}$ NMR $\left(600 \mathrm{MHz}, \mathrm{CDCl}_{3}\right) \delta$ 7.53-7.51 (m, 1H), 7.44-7.43 (m, 2H), 7.32-7.22 (m, 6H), 7.18-7.17 (m, 2H), 7.07-6.98 (m, 2H), 5.24-5.12 (m, 2H), $4.03(\mathrm{~s}, 1 \mathrm{H}), 2.23(\mathrm{~s}, 3 \mathrm{H}) .{ }^{13} \mathrm{C} \mathrm{NMR}\left(150 \mathrm{MHz}, \mathrm{CDCl}_{3}\right) \delta 175.2$, $139.9,136.7,134.2,132.0,129.6,128.9,128.1,127.3,125.5,123.9$,

$31 \mathbf{a}$ $122.8,121.6,120.7,86.4,85.8,69.1,45.3,18.6$. HRMS (ESI) $m / z$ : $[\mathrm{M}+\mathrm{H}]^{+}$Calcd for $\mathrm{C}_{24} \mathrm{H}_{20} \mathrm{NO}_{2}{ }^{+}$354.1489; Found: 354.1472 .

(R)-3la, white solid, $34.2 \mathrm{mg}$, 97\% yield (dichloromethane/ethyl acetate $=40: 1$ ), 94\% $e e,[\alpha]_{\mathrm{D}}^{26}=+28.8\left(\mathrm{c}=1.21, \mathrm{CH}_{2} \mathrm{Cl}_{2}\right)$; HPLC Daicel Chiralpak IA, n-hexane/2-propanol $=85 / 15$, flow rate $=1.0 \mathrm{~mL} / \mathrm{min}, \lambda=254 \mathrm{~nm}$, retention time (min): $13.9 \mathrm{~min}$ (major), $19.4 \mathrm{~min}$ (minor);

(S)-3la, white solid, $33.9 \mathrm{mg}, 96 \%$ yield (dichloromethane/ethyl acetate $=40: 1$ ), 83\% $e e,[\alpha]_{\mathrm{D}}^{26}=-28.7\left(\mathrm{c}=1.41, \mathrm{CH}_{2} \mathrm{Cl}_{2}\right)$; HPLC Daicel Chiralpak IA, n-hexane/2-propanol $=85 / 15$, flow rate $=1.0 \mathrm{~mL} / \mathrm{min}, \lambda=254 \mathrm{~nm}$, retention time: $14.5 \mathrm{~min}$ (minor), 20.4 min (major).
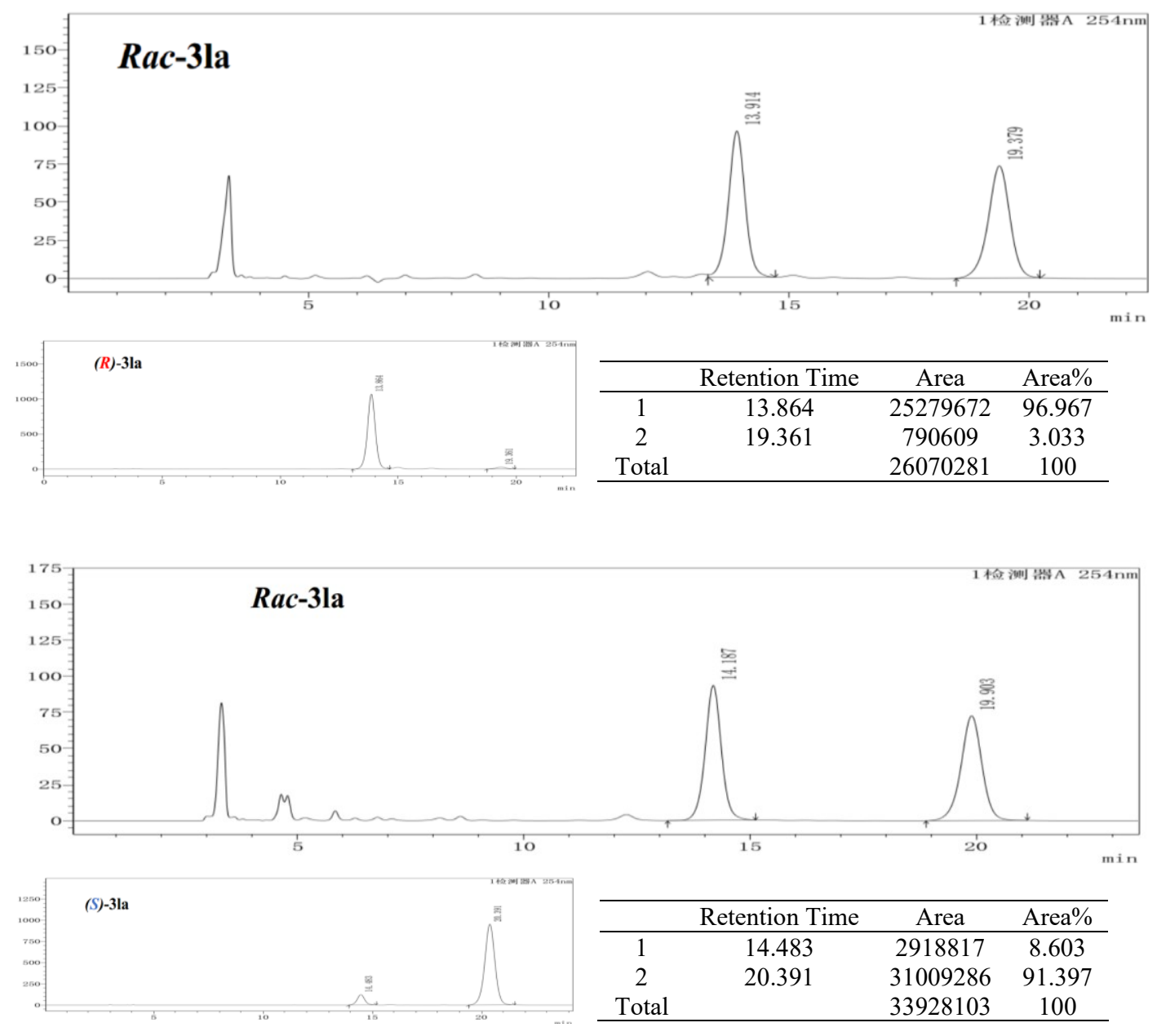


\section{1-benzyl-7-chloro-3-hydroxy-3-(phenylethynyl) indolin-2-one (3ma):}

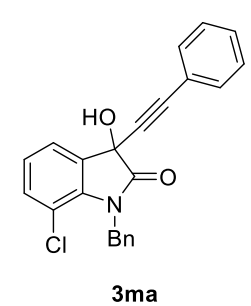

${ }^{1} \mathrm{H}$ NMR $\left(600 \mathrm{MHz}, \mathrm{CDCl}_{3}\right) \delta 7.55(\mathrm{~d}, J=7.0 \mathrm{~Hz}, 1 \mathrm{H}), 7.43(\mathrm{~d}, J=7.2$ $\mathrm{Hz}, 2 \mathrm{H}), 7.33-7.21(\mathrm{~m}, 9 \mathrm{H}), 7.06(\mathrm{t}, J=7.8 \mathrm{~Hz}, 1 \mathrm{H}), 5.39-5.33(\mathrm{~m}, 2 \mathrm{H})$, 3.93 (s, $1 \mathrm{H}) .{ }^{13} \mathrm{C} \mathrm{NMR}\left(150 \mathrm{MHz}, \mathrm{CDCl}_{3}\right) \delta 174.8,138.2,136.6,132.8$, $132.0,131.7,129.1,128.6,128.2,127.3,126.2,124.7,123.5,121.3$, $116.0,87.0,85.0,69.0,45.2$.

(R)-3ma, white solid, $32.1 \mathrm{mg}, 86 \%$ yield (dichloromethane/ethyl acetate $=50: 1$ ), $92 \%$ $e e,[\alpha]_{\mathrm{D}}^{26}=+83.0\left(\mathrm{c}=0.77, \mathrm{CH}_{2} \mathrm{Cl}_{2}\right)$; HPLC Daicel Chiralpak IA, n-hexane/2-propanol $=85 / 15$, flow rate $=1.0 \mathrm{~mL} / \mathrm{min}, \lambda=254 \mathrm{~nm}$, retention time: $10.4 \mathrm{~min}$ (major), 14.8 $\min$ (minor);

(S)-3ma, white solid, $37.0 \mathrm{mg}$, 99\% yield (dichloromethane/ethyl acetate $=50: 1$ ), 83\% $e e,[\alpha]_{\mathrm{D}}^{26}=-74.4\left(\mathrm{c}=1.12, \mathrm{CH}_{2} \mathrm{Cl}_{2}\right)\left(\operatorname{ref} .1:(S), 85 \% e e,[\alpha]_{\mathrm{D}}^{20}=-92.7(\mathrm{c}=0.56\right.$ in $\mathrm{CH}_{2} \mathrm{Cl}_{2}$ )); HPLC Daicel Chiralpak IA, n-hexane/2-propanol $=85 / 15$, flow rate $=1.0$ $\mathrm{mL} / \mathrm{min}, \lambda=254 \mathrm{~nm}$, retention time: $10.3 \mathrm{~min}$ (minor), $14.5 \mathrm{~min}$ (major).

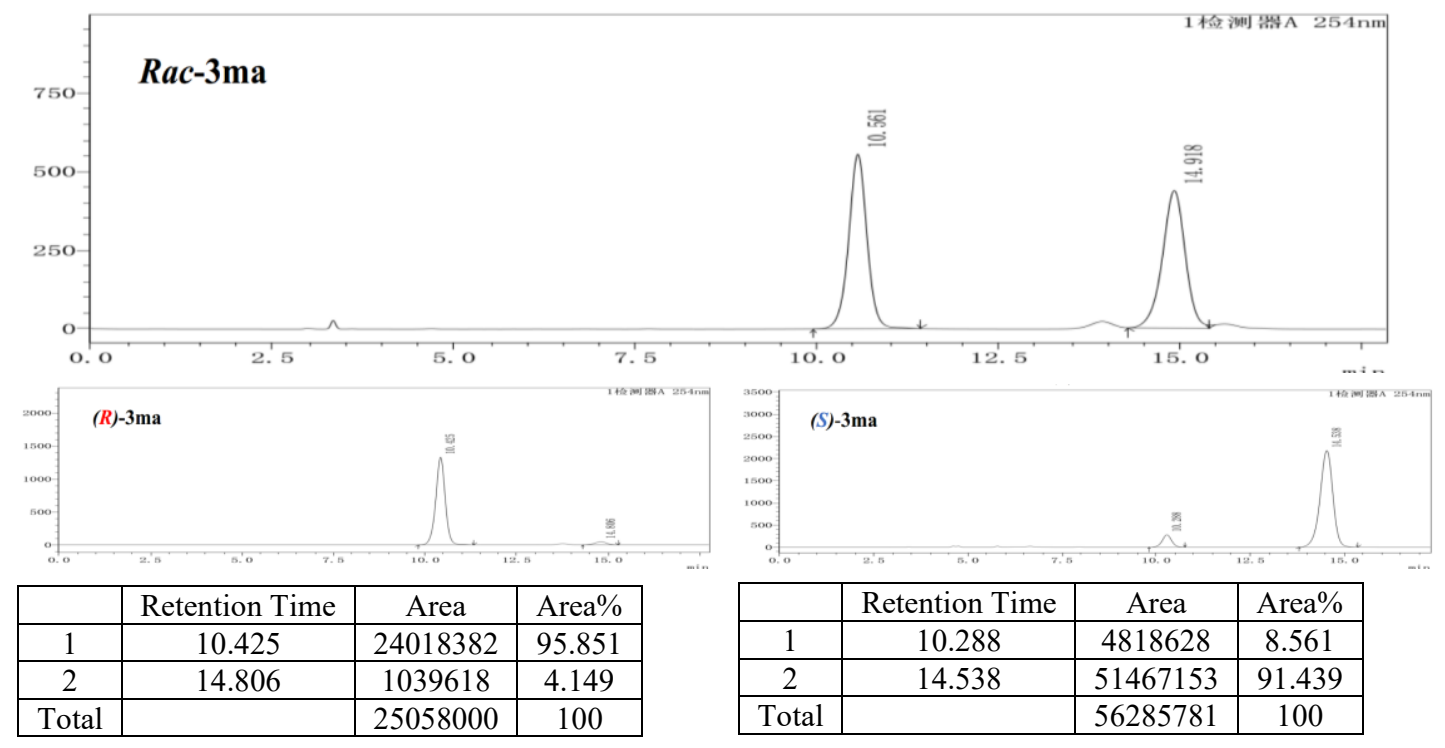




\section{1-benzyl-7-fluoro-3-hydroxy-3-(phenylethynyl) indolin-2-one (3na):}

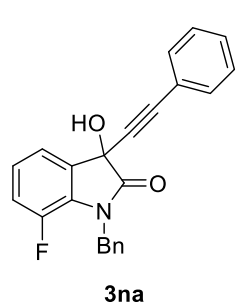

${ }^{1} \mathrm{H}$ NMR $\left(600 \mathrm{MHz}, \mathrm{CDCl}_{3}\right) \delta$ 7.46-7.39 (m, 3H), 7.36-7.22 (m, 8H), 7.09-6.97 (m, 2H), 5.12-5.00 (m, 2H), $4.16(\mathrm{~s}, 1 \mathrm{H}) .{ }^{13} \mathrm{C}$ NMR (150 $\left.\mathrm{MHz}, \mathrm{CDCl}_{3}\right) \delta 174.1,148.5,146.8,136.3,132.2,129.2,128.8,128.4$, 127.8, 127.4, 124.7 (d, $J=6.3 \mathrm{~Hz}), 121.5,120.9$ (d, $J=3.3 \mathrm{~Hz}), 118.6$ (d, $J=19.5 \mathrm{~Hz}), 87.1,85.1,69.7(\mathrm{~d}, J=2.7 \mathrm{~Hz}), 45.9$ (d, $J=4.7 \mathrm{~Hz})$.

(R)-3na, white solid, $35.3 \mathrm{mg}, 99 \%$ yield (dichloromethane/ethyl acetate $=50: 1$ ), 92\% $e e,[\alpha]_{\mathrm{D}}^{26}=-4.6\left(\mathrm{c}=1.37, \mathrm{CH}_{2} \mathrm{Cl}_{2}\right)$; HPLC Daicel Chiralpak IA, n-hexane/2-propanol = $85 / 15$, flow rate $=1.0 \mathrm{~mL} / \mathrm{min}, \lambda=254 \mathrm{~nm}$, retention time: $10.4 \mathrm{~min}$ (major), $13.2 \mathrm{~min}$ (minor);

(S)-3na, white solid, $34.3 \mathrm{mg}, 96 \%$ yield (dichloromethane/ethyl acetate $=50: 1$ ), 87\% $e e,[\alpha]_{\mathrm{D}}^{26}=+1.5\left(\mathrm{c}=1.05, \mathrm{CH}_{2} \mathrm{Cl}_{2}\right)\left(\operatorname{ref} .1:(S), 87 \% e e,[\alpha]_{\mathrm{D}}^{20}=+3.0\left(\mathrm{c}=0.51\right.\right.$ in $\left.\left.\mathrm{CH}_{2} \mathrm{Cl}_{2}\right)\right)$; HPLC Daicel Chiralpak IA, n-hexane/2-propanol $=85 / 15$, flow rate $=1.0 \mathrm{~mL} / \mathrm{min}, \lambda=$ $254 \mathrm{~nm}$, retention time: $10.3 \mathrm{~min}$ (minor), $13.0 \mathrm{~min}$ (major).

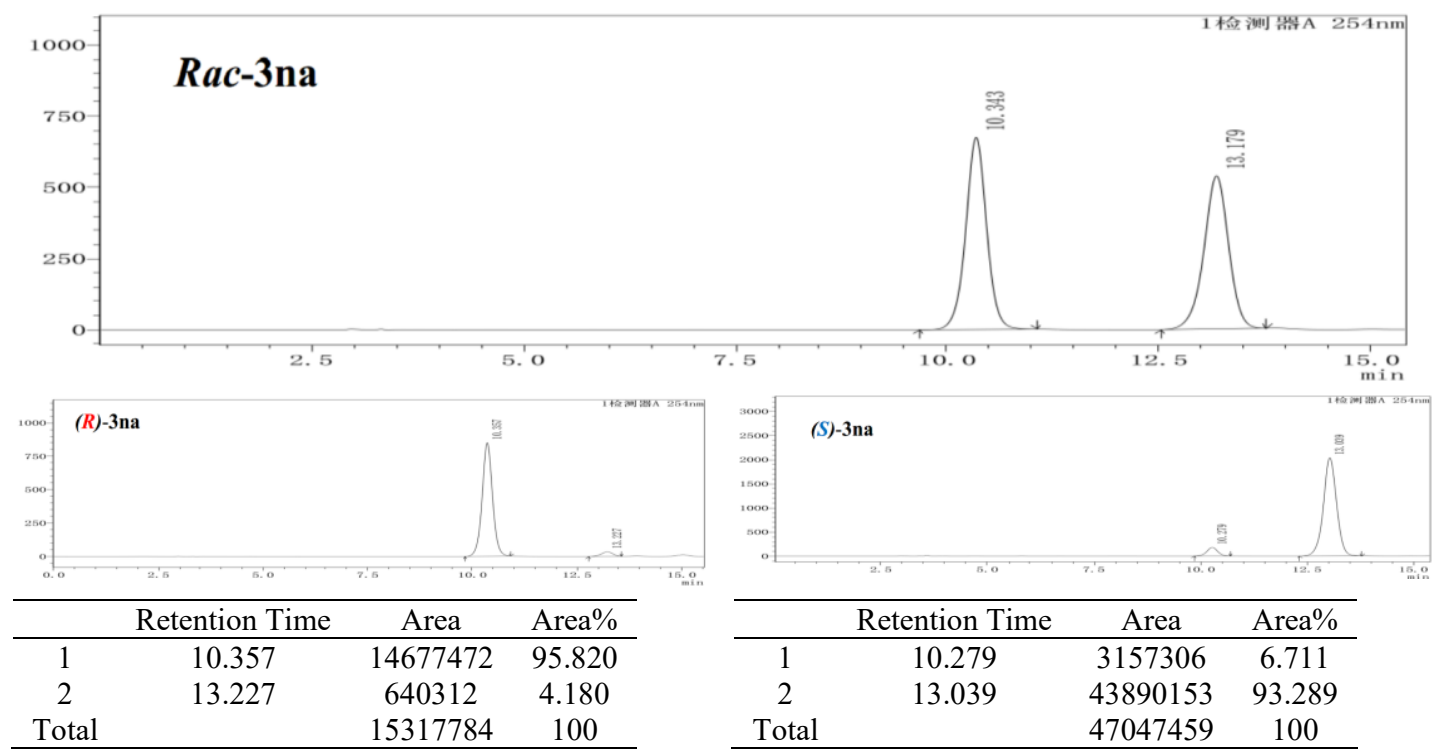




\section{1-allyl-3-hydroxy-3-(phenylethynyl) indolin-2-one (30a):}

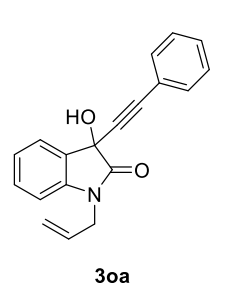

${ }^{1} \mathrm{H}$ NMR $\left(600 \mathrm{MHz}, \mathrm{CDCl}_{3}\right) \delta 7.62(\mathrm{~d}, J=6.0 \mathrm{~Hz}, 1 \mathrm{H}), 7.43(\mathrm{~d}, J=6.8$ $\mathrm{Hz}, 2 \mathrm{H}), 7.34-7.24(\mathrm{~m}, 4 \mathrm{H}), 7.15(\mathrm{t}, J=7.1 \mathrm{~Hz}, 1 \mathrm{H}), 6.85(\mathrm{~d}, J=7.1 \mathrm{~Hz}$, $1 \mathrm{H}), 5.90-5.79(\mathrm{~m}, 1 \mathrm{H}), 5.29-5.22(\mathrm{~m}, 2 \mathrm{H}), 4.38-4.31(\mathrm{~m}, 2 \mathrm{H}), 3.85(\mathrm{~s}$, 1H). ${ }^{13} \mathrm{C}$ NMR $\left(150 \mathrm{MHz}, \mathrm{CDCl}_{3}\right) \delta 173.7,142.2,132.0,130.6,130.3$, $128.9,128.1,124.7,123.7,121.6,117.9,109.7,86.4,85.5,69.5,42.6$.

(R)-3oa, white solid, $23.4 \mathrm{mg}, 81 \%$ yield (dichloromethane/ethyl acetate $=50: 1$ ), 96\% $e e,[\alpha]_{\mathrm{D}}^{26}=-9.2\left(\mathrm{c}=0.87, \mathrm{CH}_{2} \mathrm{Cl}_{2}\right)$; HPLC Daicel Chiralpak IA, n-hexane/2-propanol = $90 / 10$, flow rate $=1.0 \mathrm{~mL} / \mathrm{min}, \lambda=254 \mathrm{~nm}$, retention time: $13.1 \mathrm{~min}$ (major), $14.6 \mathrm{~min}$ (minor);

(S)-3oa, white solid, $26.9 \mathrm{mg}, 93 \%$ yield (dichloromethane/ethyl acetate $=50: 1$ ), 87\% $e e,[\alpha]_{\mathrm{D}}^{26}=+9.7\left(\mathrm{c}=0.79, \mathrm{CH}_{2} \mathrm{Cl}_{2}\right)\left(\operatorname{ref} .1:(S), 95 \% e e,[\alpha]_{\mathrm{D}}^{20}=+9.3\left(\mathrm{c}=0.51\right.\right.$ in $\left.\left.\mathrm{CH}_{2} \mathrm{Cl}_{2}\right)\right)$; HPLC Daicel Chiralpak IA, n-hexane/2-propanol $=90 / 10$, flow rate $=1.0 \mathrm{~mL} / \mathrm{min}, \lambda=$ $254 \mathrm{~nm}$, retention time: $13.1 \mathrm{~min}$ (minor), $14.4 \mathrm{~min}$ (major).

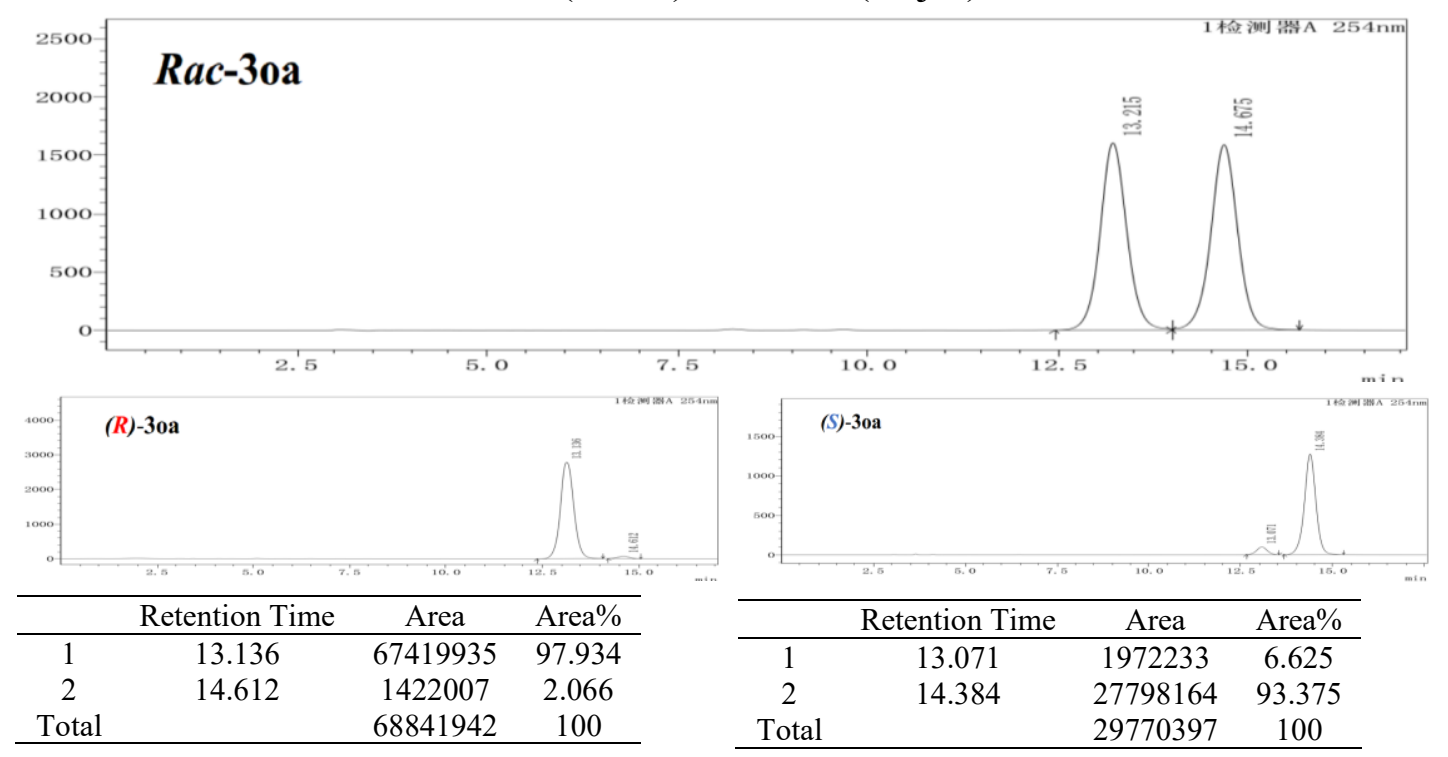




\section{3-Hydroxy-3-(phenylethynyl) indolin-2-one (3pa):}

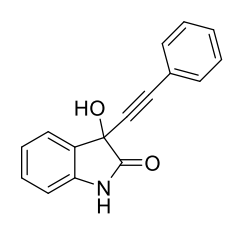

$3 \mathrm{pa}$

${ }^{1} \mathrm{H}$ NMR (600 MHz, DMSO-d $d_{6} \delta 10.57(\mathrm{~s}, 1 \mathrm{H}), 7.46(\mathrm{~d}, J=7.4 \mathrm{~Hz}$, 1H), 7.44-7.34 (m, 5H), 7.28 (t, $J=7.7 \mathrm{~Hz}, 1 \mathrm{H}), 7.09$ (s, 1H), 7.05 (t, $J$ $=7.5 \mathrm{~Hz}, 1 \mathrm{H}), 6.87(\mathrm{~d}, J=7.7 \mathrm{~Hz}, 1 \mathrm{H}) .{ }^{13} \mathrm{C} \mathrm{NMR}(150 \mathrm{MHz}, \mathrm{DMSO}-$ $\left.d_{6}\right) \delta 175.0,141.6,131.9,131.4,130.4,129.5,129.1,124.9,122.8$, $121.8,110.6,88.4,84.5,69.5$.

$(R)-3$ pa, white solid, $18.7 \mathrm{mg}, 75 \%$ yield (dichloromethane/ethyl acetate $=30: 1$ ), 95\% $e e,[\alpha]_{\mathrm{D}}^{26}=+38.5(\mathrm{c}=0.14, \mathrm{MeOH})$; HPLC: Daicel Chiralpak IA, n-hexane/2-propanol $=85 / 15$, flow rate $=1.0 \mathrm{~mL} / \mathrm{min}, \lambda=254 \mathrm{~nm}$, retention time: 11.6 min (major), 15.5 $\min$ (minor);

(S)-3pa, white solid, $18.2 \mathrm{mg}, 73 \%$ yield (dichloromethane/ethyl acetate $=30: 1$ ), 91\% $e e,[\alpha]_{\mathrm{D}}^{26}=-33.7(\mathrm{c}=0.10, \mathrm{MeOH})\left(\operatorname{ref} .3:(S), 99 \% e e,[\alpha]_{\mathrm{D}}^{20}=-35.0(c 0.5, \mathrm{MeOH})\right)$; HPLC: Daicel Chiralpak IA, n-hexane/2-propanol $=85 / 15$, flow rate $=1.0 \mathrm{~mL} / \mathrm{min}, \lambda$ $=254 \mathrm{~nm}$, retention time: $11.2 \mathrm{~min}$ (minor), $15.2 \mathrm{~min}$ (major).
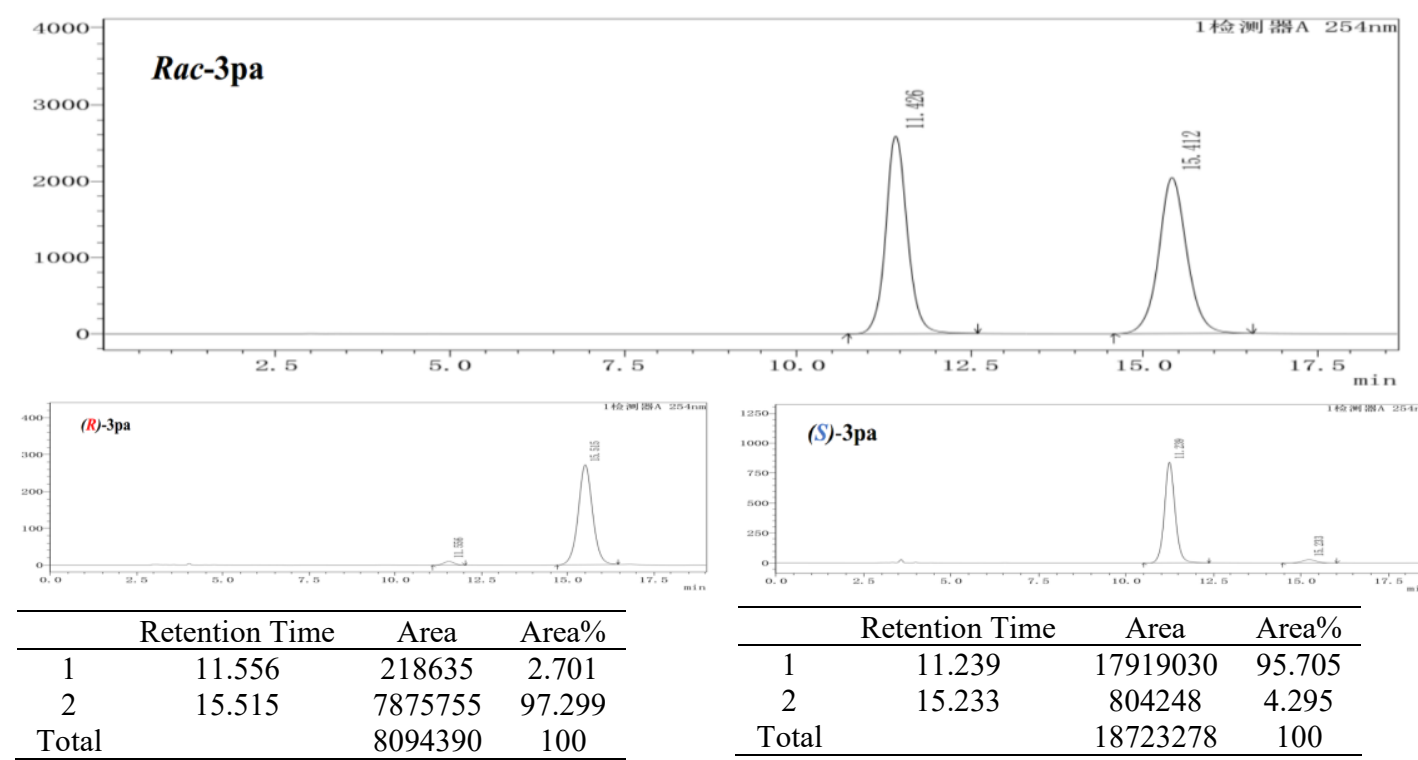


\section{1-benzyl-3-(hex-1-yn-1-yl)-3-hydroxyindolin-2-one (3ab):}

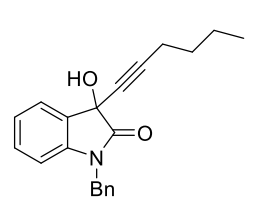

$3 a b$

${ }^{1} \mathrm{H}$ NMR $\left(600 \mathrm{MHz}, \mathrm{CDCl}_{3}\right) \delta 7.52(\mathrm{~d}, J=7.3 \mathrm{~Hz}, 1 \mathrm{H}), 7.31-7.25(\mathrm{~m}$, $5 \mathrm{H}), 7.20(\mathrm{t}, J=8.3 \mathrm{~Hz}, 1 \mathrm{H}), 7.08(\mathrm{t}, J=7.5 \mathrm{~Hz}, 1 \mathrm{H}), 6.68(\mathrm{~d}, J=7.9$ $\mathrm{Hz}, 1 \mathrm{H}), 4.97-4.81(\mathrm{~m}, 2 \mathrm{H}), 3.53(\mathrm{~s}, 1 \mathrm{H}), 2.24(\mathrm{t}, J=7.2 \mathrm{~Hz}, 2 \mathrm{H})$, 1.52-1.36 (m, 4H), $0.89(\mathrm{t}, J=7.4 \mathrm{~Hz}, 3 \mathrm{H}) .{ }^{13} \mathrm{C} \mathrm{NMR}(150 \mathrm{MHz}$, $\left.\mathrm{CDCl}_{3}\right) \delta 174.4,142.0,135.1,130.1,129.3,128.8,127.7,127.1,124.5,123.6,109.7$, 88.2, 69.2, 43.9, 30.2, 21.9, 18.5, 13.5 .

$(R)-3 \mathbf{a b}$, white solid, $22.6 \mathrm{mg}, 71 \%$ yield (dichloromethane/ethyl acetate $=40: 1$ ), $92 \%$ $e e,[\alpha]_{\mathrm{D}}^{26}=-23.5\left(\mathrm{c}=0.43, \mathrm{CH}_{2} \mathrm{Cl}_{2}\right)$; HPLC: Daicel Chiralpak IA, n-hexane/2-propanol $=90 / 10$, flow rate $=1.0 \mathrm{~mL} / \mathrm{min}, \lambda=254 \mathrm{~nm}$, retention time: $11.7 \mathrm{~min}$ (major), 12.9 $\min$ (minor);

(S)-3ab, white solid, $23.9 \mathrm{mg}, 75 \%$ yield (dichloromethane/ethyl acetate $=40: 1$ ), 82\% $e e,[\alpha]_{\mathrm{D}}^{26}=+20.4\left(\mathrm{c}=0.29, \mathrm{CH}_{2} \mathrm{Cl}_{2}\right)\left(\operatorname{ref} .1:(S), 96 \% e e,[\alpha]_{\mathrm{D}}^{20}=+21.6(\mathrm{c}=0.52\right.$ in $\mathrm{CH}_{2} \mathrm{Cl}_{2}$ )); HPLC: Daicel Chiralpak IA, n-hexane/2-propanol $=90 / 10$, flow rate $=1.0$ $\mathrm{mL} / \mathrm{min}, \lambda=254 \mathrm{~nm}$, retention time: $11.6 \mathrm{~min}$ (minor), $12.7 \mathrm{~min}$ (major).

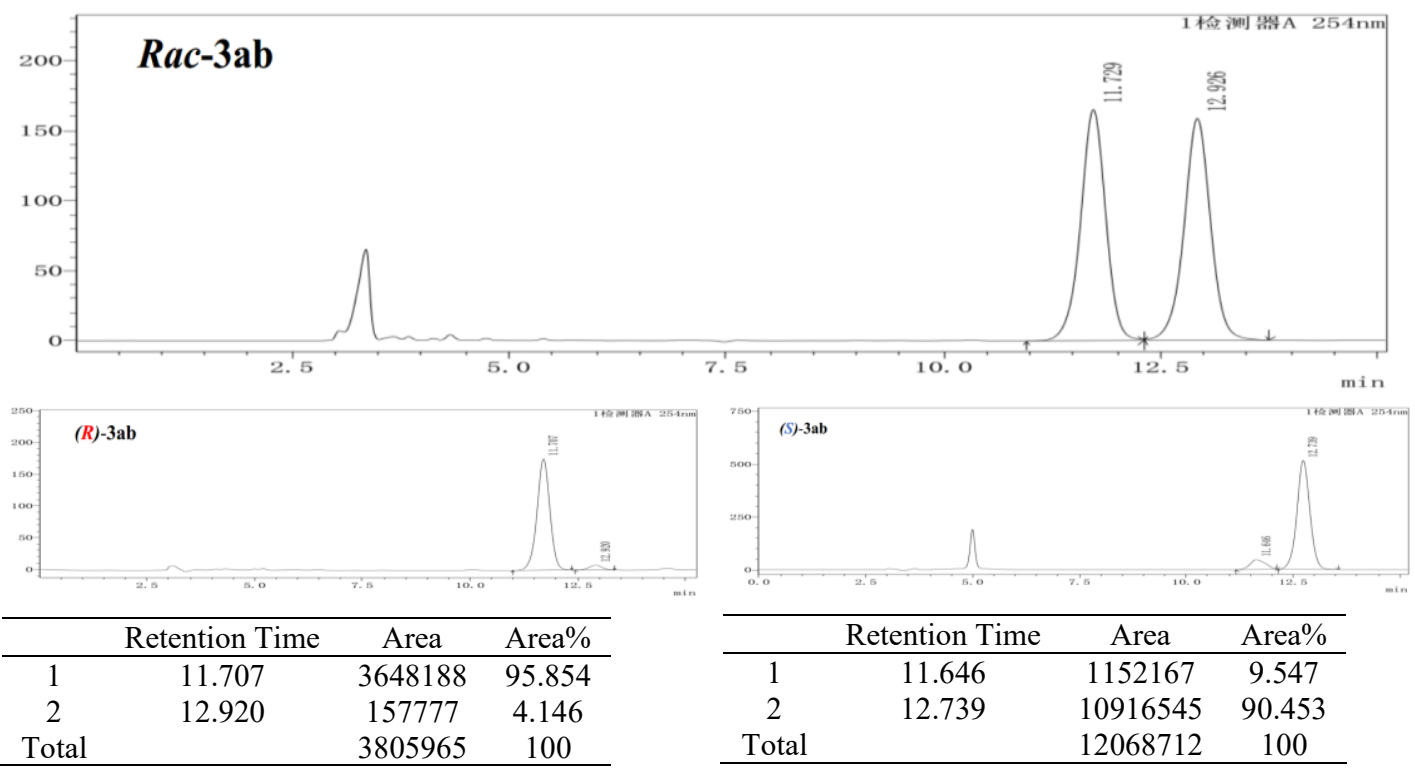




\section{1-benzyl-3-((2-fluorophenyl) ethynyl)-3-hydroxyindolin-2-one (3ac):}

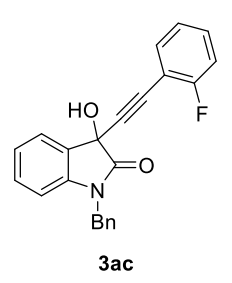

${ }^{1} \mathrm{H}$ NMR $\left(600 \mathrm{MHz}, \mathrm{CDCl}_{3}\right) \delta 7.62(\mathrm{~d}, J=7.1 \mathrm{~Hz}, 1 \mathrm{H}), 7.44-7.41(\mathrm{~m}$, $1 \mathrm{H}), 7.32-7.22(\mathrm{~m}, 7 \mathrm{H}), 7.10(\mathrm{t}, J=7.5 \mathrm{~Hz}, 1 \mathrm{H}), 7.04-7.00(\mathrm{~m}, 2 \mathrm{H})$, $6.71(\mathrm{~d}, J=7.9 \mathrm{~Hz}, 1 \mathrm{H}), 4.98-4.85(\mathrm{~m}, 2 \mathrm{H}), 4.11(\mathrm{~s}, 1 \mathrm{H}) .{ }^{13} \mathrm{C} \mathrm{NMR}(150$ $\left.\mathrm{MHz}, \mathrm{CDCl}_{3}\right) \delta 173.9,163.8,162.1,142.1,134.9,133.8,130.7(\mathrm{~d}, J=$ $8.0 \mathrm{~Hz}), 130.4,128.8,128.7,127.7,127.1,124.8,123.8$ (d, $J=4.6 \mathrm{~Hz})$, $115.5,115.3,110.3$ (d, $J=15.5 \mathrm{~Hz}), 109.9,90.5$ (d, $J=3.3 \mathrm{~Hz}), 80.0$, 69.7, 44.0.

$(R)$-3ac, white solid, $31.8 \mathrm{mg}, 89 \%$ yield (dichloromethane/ethyl acetate $=50: 1$ ), 92\% $e e,[\alpha]_{\mathrm{D}}^{26}=-12.0\left(\mathrm{c}=0.54, \mathrm{CH}_{2} \mathrm{Cl}_{2}\right)$; HPLC: Daicel Chiralpak IA, n-hexane/2-propanol $=85 / 15$, flow rate $=1.0 \mathrm{~mL} / \mathrm{min}, \lambda=254 \mathrm{~nm}$, retention time: $13.9 \min$ (major), 16.3 $\min$ (minor);

(S)-3ac, white solid, $30.7 \mathrm{mg}, 86 \%$ yield (dichloromethane/ethyl acetate $=50: 1$ ), 85\% $e e,[\alpha]_{\mathrm{D}}^{26}=+16.2\left(\mathrm{c}=1.15, \mathrm{CH}_{2} \mathrm{Cl}_{2}\right)\left(\operatorname{ref} .1:(S), 95 \% e e,[\alpha]_{\mathrm{D}}^{20}=+17.0(\mathrm{c}=0.54\right.$ in $\left.\mathrm{CH}_{2} \mathrm{Cl}_{2}\right)$ ); HPLC: Daicel Chiralpak IA, n-hexane/2-propanol $=85 / 15$, flow rate $=1.0$ $\mathrm{mL} / \mathrm{min}, \lambda=254 \mathrm{~nm}$, retention time: $13.8 \mathrm{~min}$ (minor), $16.0 \mathrm{~min}$ (major).

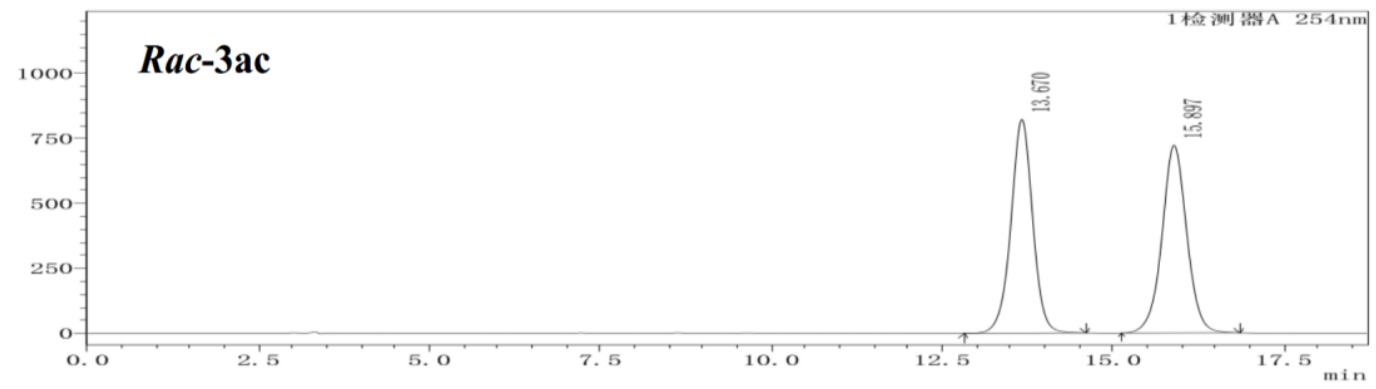

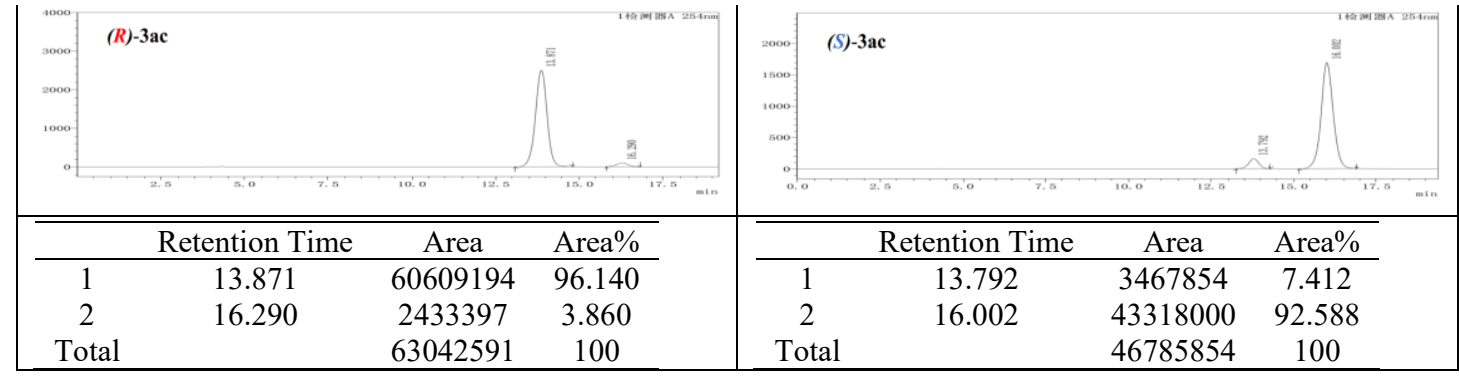




\section{1-benzyl-3-hydroxy-3-(m-tolylethynyl) indolin-2-one (3ad):}

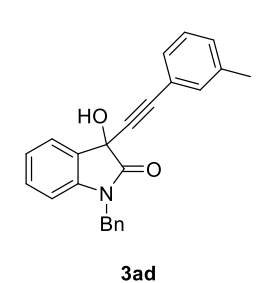

${ }^{1} \mathrm{H}$ NMR $\left(600 \mathrm{MHz}, \mathrm{CDCl}_{3}\right) \delta 7.62(\mathrm{t}, J=6.8 \mathrm{~Hz}, 1 \mathrm{H}), 7.30-7.20(\mathrm{~m}$, $8 \mathrm{H}), 7.17-7.07(\mathrm{~m}, 3 \mathrm{H}), 6.70(\mathrm{~d}, J=7.7 \mathrm{~Hz}, 1 \mathrm{H}), 4.97-4.84(\mathrm{~m}, 2 \mathrm{H})$, $4.11(\mathrm{~s}, 1 \mathrm{H}), 2.26(\mathrm{~s}, 3 \mathrm{H}) .{ }^{13} \mathrm{C} \mathrm{NMR}\left(150 \mathrm{MHz}, \mathrm{CDCl}_{3}\right) \delta 174.2,142.0$, 137.8, 135.0, 132.6, 130.2, 129.8, 129.1, 129.0, 128.8, 128.0, 127.7, 127.1, 124.7, 123.7, 121.4, 109.8, 86.7, 85.1, 69.6, 44.0, 21.1.

(R)-3ad, white solid, $32.8 \mathrm{mg}, 93 \%$ yield (dichloromethane/ethyl acetate $=50: 1$ ), $91 \%$ $e e,[\alpha]_{\mathrm{D}}^{26}=-13.5\left(\mathrm{c}=0.91, \mathrm{CH}_{2} \mathrm{Cl}_{2}\right)$; HPLC: Daicel Chiralpak IA, n-hexane/2-propanol $=90 / 10$, flow rate $=1.0 \mathrm{~mL} / \mathrm{min}, \lambda=254 \mathrm{~nm}$, retention time: $15.5 \mathrm{~min}$ (major), 16.9 $\min$ (minor);

(S)-3ad, white solid, $33.9 \mathrm{mg}, 96 \%$ yield (dichloromethane/ethyl acetate $=50: 1$ ), 82\% $e e,[\alpha]_{\mathrm{D}}^{26}=+13.5\left(\mathrm{c}=0.89, \mathrm{CH}_{2} \mathrm{Cl}_{2}\right)$; HPLC: Daicel Chiralpak IA, n-hexane/2-propanol $=90 / 10$, flow rate $=1.0 \mathrm{~mL} / \mathrm{min}, \lambda=254 \mathrm{~nm}$, retention time: $15.5 \mathrm{~min}$ (minor), 16.7 $\min$ (major).

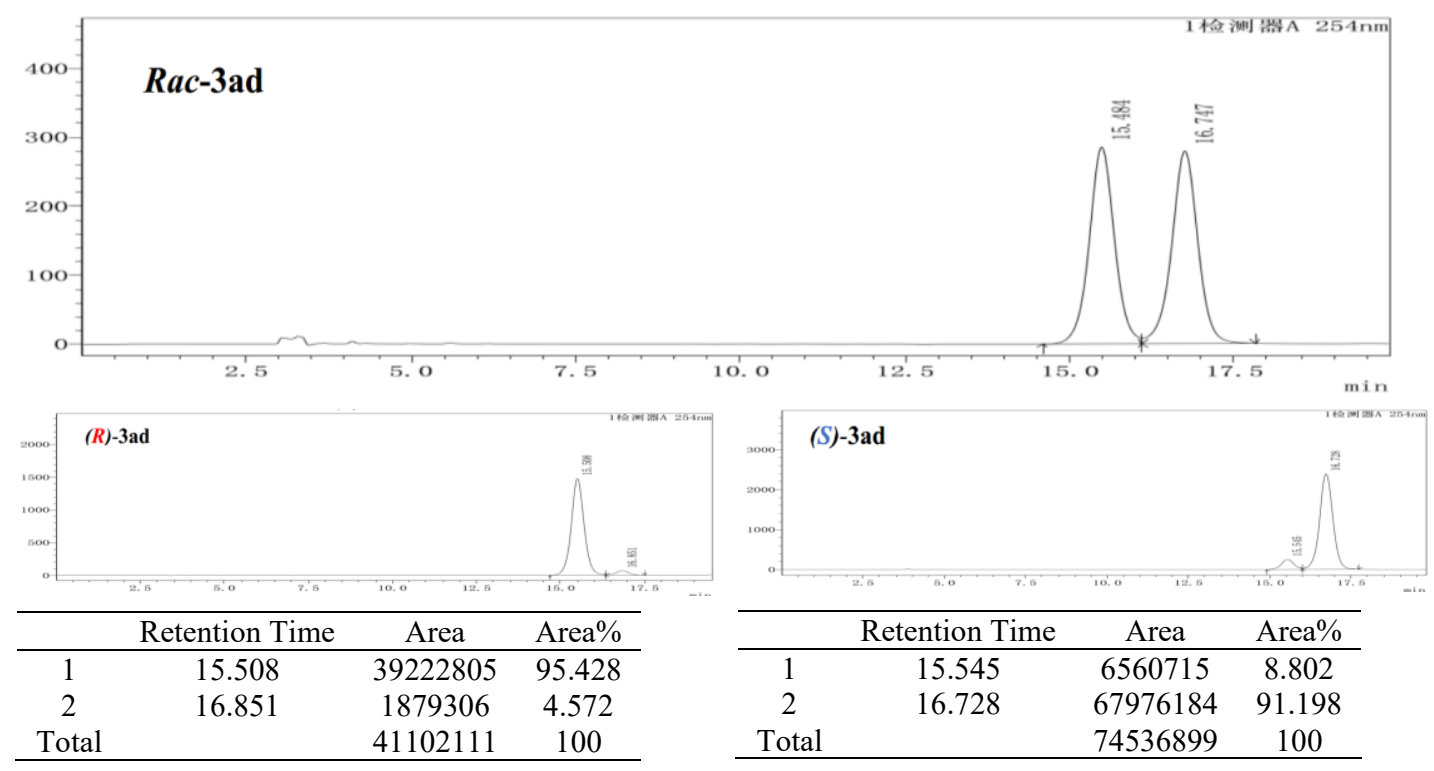




\section{1-benzyl-3-((3-chlorophenyl) ethynyl)-3-hydroxyindolin-2-one (3ae):}

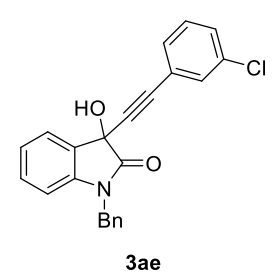

${ }^{1} \mathrm{H}$ NMR $\left(600 \mathrm{MHz}, \mathrm{CDCl}_{3}\right) \delta 7.61(\mathrm{~d}, J=6.6 \mathrm{~Hz}, 1 \mathrm{H}), 7.44-7.38(\mathrm{~m}$, $1 \mathrm{H}), 7.31-7.22(\mathrm{~m}, 8 \mathrm{H}), 7.17-7.10(\mathrm{~m}, 2 \mathrm{H}), 6.72(\mathrm{~d}, J=7.8 \mathrm{~Hz}, 1 \mathrm{H})$, 4.97-4.87 (m, 2H), $4.46(\mathrm{~s}, 1 \mathrm{H}) .{ }^{13} \mathrm{C} \mathrm{NMR}\left(150 \mathrm{MHz}, \mathrm{CDCl}_{3}\right) \delta 174.1$, $142.0,134.9,134.0,131.8,130.4,130.1,129.4,129.2,128.8,128.7$, $127.8,127.1,124.8,123.8,123.3,109.9,86.7,84.9,69.6,44.1$.

( $R$ )-3ae, white solid, $31.7 \mathrm{mg}, 85 \%$ yield (dichloromethane/ethyl acetate $=50: 1$ ), $94 \%$ $e e,[\alpha]_{\mathrm{D}}^{26}=-9.8\left(\mathrm{c}=2.05, \mathrm{CH}_{2} \mathrm{Cl}_{2}\right)$; HPLC: Daicel Chiralpak IA, n-hexane/2-propanol = $85 / 15$, flow rate $=1.0 \mathrm{~mL} / \mathrm{min}, \lambda=254 \mathrm{~nm}$, retention time: $11.0 \mathrm{~min}$ (major), $13.1 \mathrm{~min}$ (minor);

(S)-3ae, white solid, $32.5 \mathrm{mg}, 87 \%$ yield (dichloromethane/ethyl acetate $=50: 1$ ), $91 \%$ $e e,[\alpha]_{\mathrm{D}}^{26}=+6.3\left(\mathrm{c}=0.51, \mathrm{CH}_{2} \mathrm{Cl}_{2}\right)\left(\operatorname{ref} .1:(S), 93 \% e e,[\alpha]_{\mathrm{D}}^{20}=+11.3(\mathrm{c}=0.56\right.$ in $\mathrm{CH}_{2} \mathrm{Cl}_{2}$ )); HPLC: Daicel Chiralpak IA, n-hexane/2-propanol $=85 / 15$, flow rate $=1.0$ $\mathrm{mL} / \mathrm{min}, \lambda=254 \mathrm{~nm}$, retention time: $10.9 \mathrm{~min}$ (minor), $13.0 \mathrm{~min}$ (major).
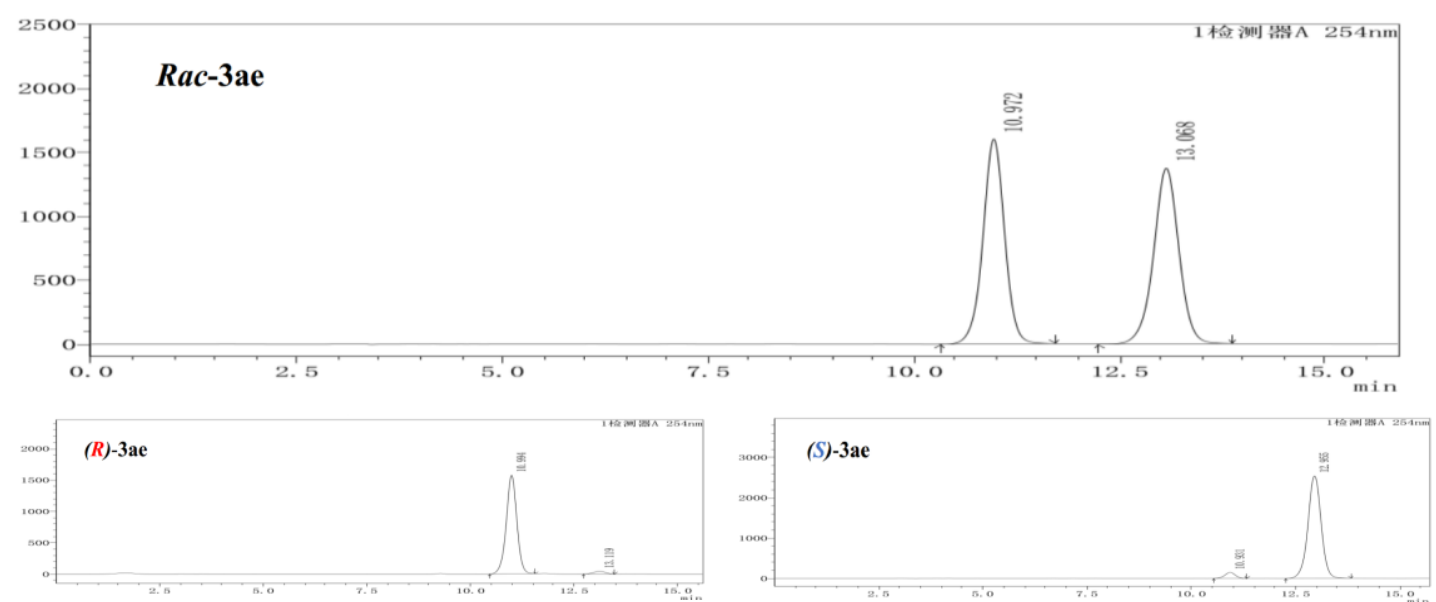

\begin{tabular}{cccc}
\hline & Retention Time & Area & Area\% \\
\hline 1 & 10.994 & 28044465 & 97.052 \\
2 & 13.119 & 851768 & 2.948 \\
Total & & 28896233 & 100 \\
\hline
\end{tabular}

\begin{tabular}{cccc}
\hline & Retention Time & Area & Area\% \\
\hline 1 & 10.931 & 2577195 & 4.400 \\
2 & 12.955 & 55990690 & 95.600 \\
Total & & 58567885 & 100 \\
\hline
\end{tabular}




\section{1-benzyl-3-((3-fluorophenyl) ethynyl)-3-hydroxyindolin-2-one (3af):}

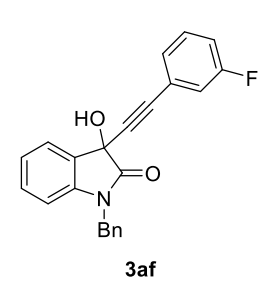

${ }^{1} \mathrm{H}$ NMR $\left(600 \mathrm{MHz}, \mathrm{CDCl}_{3}\right) \delta 7.61(\mathrm{~d}, J=7.3 \mathrm{~Hz}, 1 \mathrm{H}), 7.31-7.19(\mathrm{~m}$, $8 \mathrm{H}), 7.12-7.11(\mathrm{~m}, 2 \mathrm{H}), 7.04-6.97(\mathrm{~m}, 1 \mathrm{H}), 6.72(\mathrm{~d}, J=7.9 \mathrm{~Hz}, 1 \mathrm{H})$, 4.97-4.87 (m, 2H), $4.30(\mathrm{~s}, 1 \mathrm{H}) .{ }^{13} \mathrm{C} \mathrm{NMR}\left(150 \mathrm{MHz}, \mathrm{CDCl}_{3}\right) \delta 174.1$, $162.9,161.3,142.0,134.9,130.4,129.8$ (d, $J=8.5 \mathrm{~Hz}), 128.8,128.7$, $127.9(\mathrm{~d}, J=3.1 \mathrm{~Hz}), 127.8,127.1,124.8,123.8,123.4(\mathrm{~d}, J=9.5$

Hz), 118.9, 118.7, 116.4, 116.2, 109.9, 86.4, 85.1 (d, $J=3.4 \mathrm{~Hz}), 69.6,44.1$.

$(R)$-3af, white solid, $32.5 \mathrm{mg}, 91 \%$ yield (dichloromethane/ethyl acetate $=50: 1$ ), 95\% $e e,[\alpha]_{\mathrm{D}}^{26}=-17.6\left(\mathrm{c}=0.63, \mathrm{CH}_{2} \mathrm{Cl}_{2}\right)$; HPLC: Daicel Chiralpak IA, n-hexane/2-propanol $=85 / 15$, flow rate $=1.0 \mathrm{~mL} / \mathrm{min}, \lambda=254 \mathrm{~nm}$, retention time: $10.9 \min$ (major), 13.3 $\min$ (minor),

(S)-3af, white solid, $33.2 \mathrm{mg}, 93 \%$ yield (dichloromethane/ethyl acetate $=50: 1$ ), 92\% $e e,[\alpha]_{\mathrm{D}}^{26}=+14.9\left(\mathrm{c}=0.86, \mathrm{CH}_{2} \mathrm{Cl}_{2}\right)\left(\operatorname{ref} .1:(S), 94 \% e e,[\alpha]_{\mathrm{D}}^{20}=+12.3(\mathrm{c}=0.57\right.$ in $\left.\mathrm{CH}_{2} \mathrm{Cl}_{2}\right)$ ); HPLC: Daicel Chiralpak IA, n-hexane/2-propanol $=85 / 15$, flow rate $=1.0$ $\mathrm{mL} / \mathrm{min}, \lambda=254 \mathrm{~nm}$, retention time: $10.9 \mathrm{~min}$ (minor), $13.3 \mathrm{~min}$ (major).

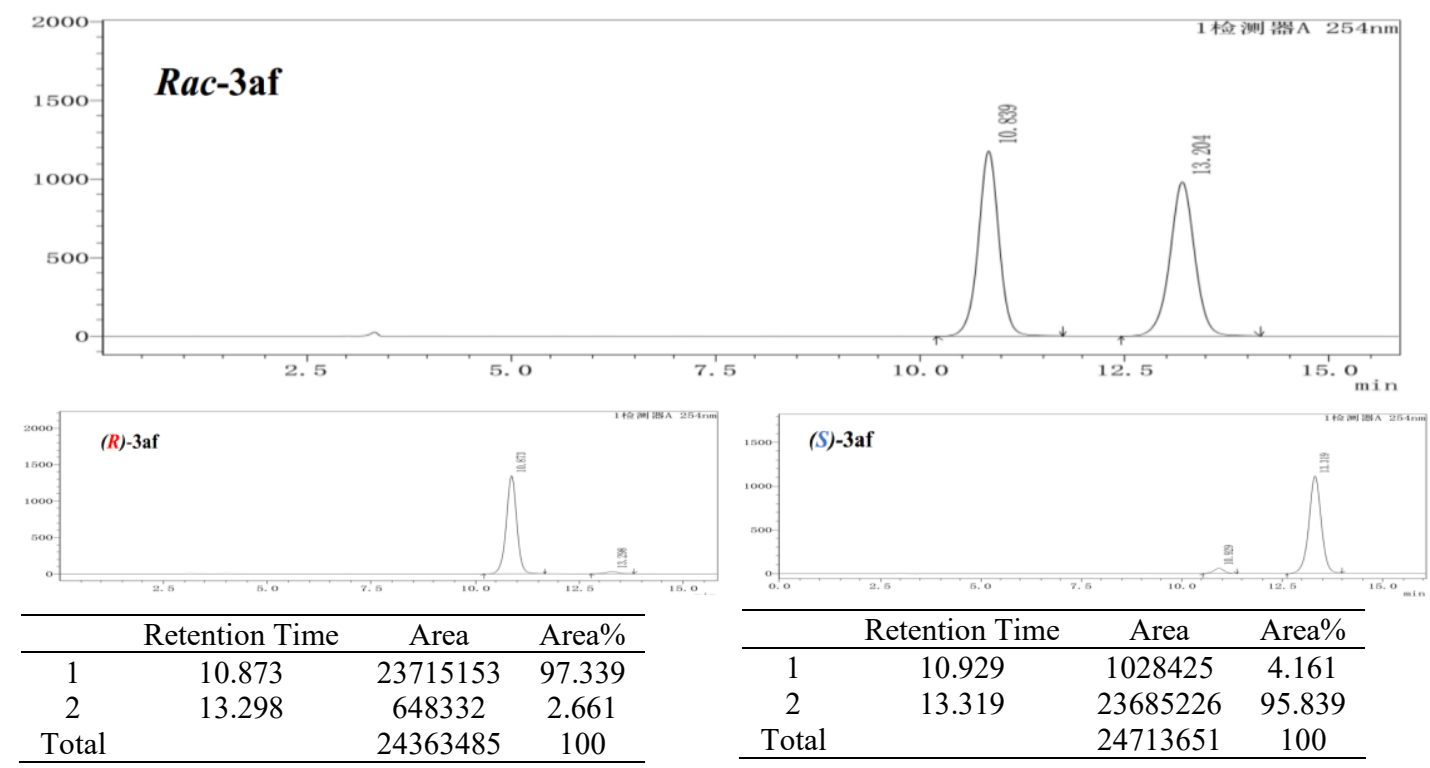




\section{1-benzyl-3-((4-bromophenyl) ethynyl)-3-hydroxyindolin-2-one (3ag):}

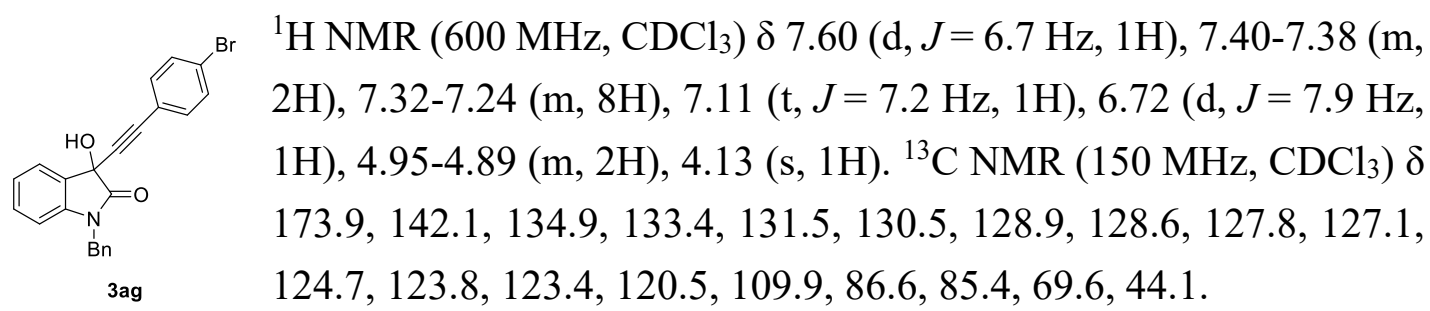

( $R$ )-3ag, white solid, $36.7 \mathrm{mg}, 88 \%$ yield (dichloromethane/ethyl acetate $=50: 1$ ), $93 \%$ $e e,[\alpha]_{\mathrm{D}}^{26}=-9.3\left(\mathrm{c}=0.75, \mathrm{CH}_{2} \mathrm{Cl}_{2}\right)$; HPLC: Daicel Chiralpak IA, n-hexane/2-propanol = $85 / 15$, flow rate $=1.0 \mathrm{~mL} / \mathrm{min}, \lambda=254 \mathrm{~nm}$, retention time: $15.2 \mathrm{~min}$ (major), $19.0 \mathrm{~min}$ (minor);

(S)-3ag, white solid, $37.5 \mathrm{mg}, 90 \%$ yield (dichloromethane/ethyl acetate $=50: 1$ ), 91\% $e e,[\alpha]_{\mathrm{D}}^{26}=+10.3\left(\mathrm{c}=0.54, \mathrm{CH}_{2} \mathrm{Cl}_{2}\right)\left(\operatorname{ref} .1:(S), 93 \% e e,[\alpha]_{\mathrm{D}}^{20}=+12.6(\mathrm{c}=0.50\right.$ in $\mathrm{CH}_{2} \mathrm{Cl}_{2}$ )); HPLC: Daicel Chiralpak IA, n-hexane/2-propanol $=85 / 15$, flow rate $=1.0$ $\mathrm{mL} / \mathrm{min}, \lambda=254 \mathrm{~nm}$, retention time: $15.0 \mathrm{~min}$ (minor), $18.5 \mathrm{~min}$ (major).

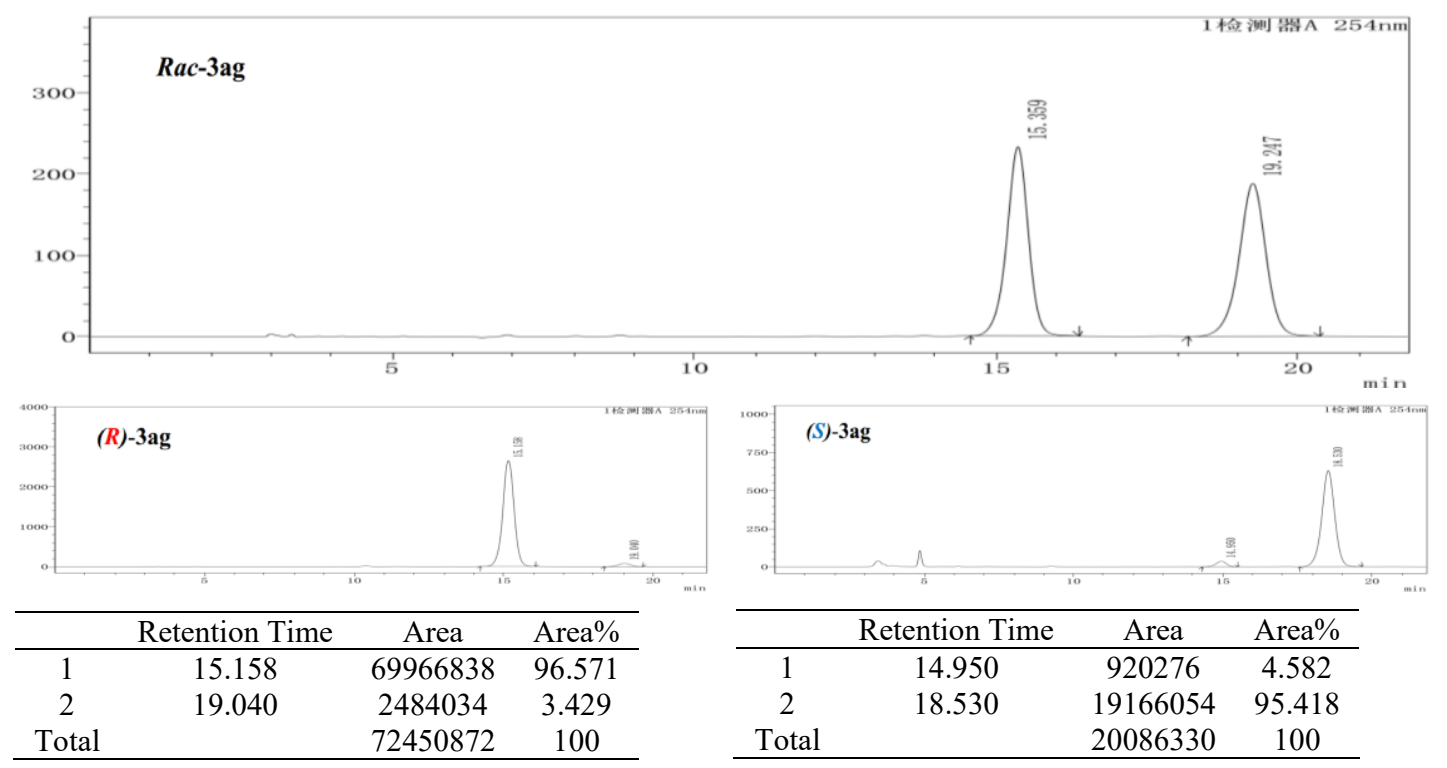




\section{1-benzyl-3-hydroxy-3-(p-tolylethynyl) indolin-2-one (3ah):}

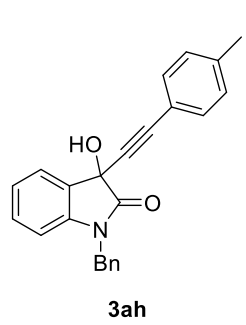

${ }^{1} \mathrm{H}$ NMR $\left(600 \mathrm{MHz}, \mathrm{CDCl}_{3}\right) \delta 7.61(\mathrm{~d}, J=7.4 \mathrm{~Hz}, 1 \mathrm{H}), 7.36-7.28(\mathrm{~m}$, $6 \mathrm{H}), 7.26-7.22(\mathrm{~m}, 2 \mathrm{H}), 7.14-7.04(\mathrm{~m}, 3 \mathrm{H}), 6.71(\mathrm{~d}, J=7.8 \mathrm{~Hz}, 1 \mathrm{H})$, $4.92(\mathrm{~s}, 2 \mathrm{H}), 3.83$ (s, 1H), 2.33 (s, 3H). ${ }^{13} \mathrm{C} \mathrm{NMR}\left(150 \mathrm{MHz}, \mathrm{CDCl}_{3}\right)$ $\delta 174.1,142.1,139.2,135.0,132.0,130.3,128.9,128.8,127.7,127.1$, $124.7,123.7,118.5,109.8,86.8,84.7,69.6,44.0,21.5$.

(R)-3ah, white solid, $34.2 \mathrm{mg}, 97 \%$ yield (dichloromethane/ethyl acetate $=40: 1), 92 \% e e,[\alpha]_{\mathrm{D}}^{26}=-19.6\left(c=0.97, \mathrm{CH}_{2} \mathrm{Cl}_{2}\right)$; HPLC: Daicel Chiralpak IA, n-hexane $/ 2$-propanol $=85 / 15$, flow rate $=1.0 \mathrm{~mL} / \mathrm{min}, \lambda=254 \mathrm{~nm}$, retention time: 13.5 min (major), $15.8 \mathrm{~min}$ (minor);

(S)-3ah, white solid, $33.5 \mathrm{mg}, 95 \%$ yield (dichloromethane/ethyl acetate $=40: 1$ ), $82 \%$ $e e,[\alpha]_{\mathrm{D}}^{26}=+15.7\left(\mathrm{c}=0.61, \mathrm{CH}_{2} \mathrm{Cl}_{2}\right)\left(\operatorname{ref} .1:(S), 91 \% e e,[\alpha]_{\mathrm{D}}^{20}=+17.6(\mathrm{c}=0.52\right.$ in $\mathrm{CH}_{2} \mathrm{Cl}_{2}$ )); HPLC: Daicel Chiralpak IA, n-hexane/2-propanol $=85 / 15$, flow rate $=1.0$ $\mathrm{mL} / \mathrm{min}, \lambda=254 \mathrm{~nm}$, retention time: $13.7 \mathrm{~min}$ (minor), $15.8 \mathrm{~min}$ (major).
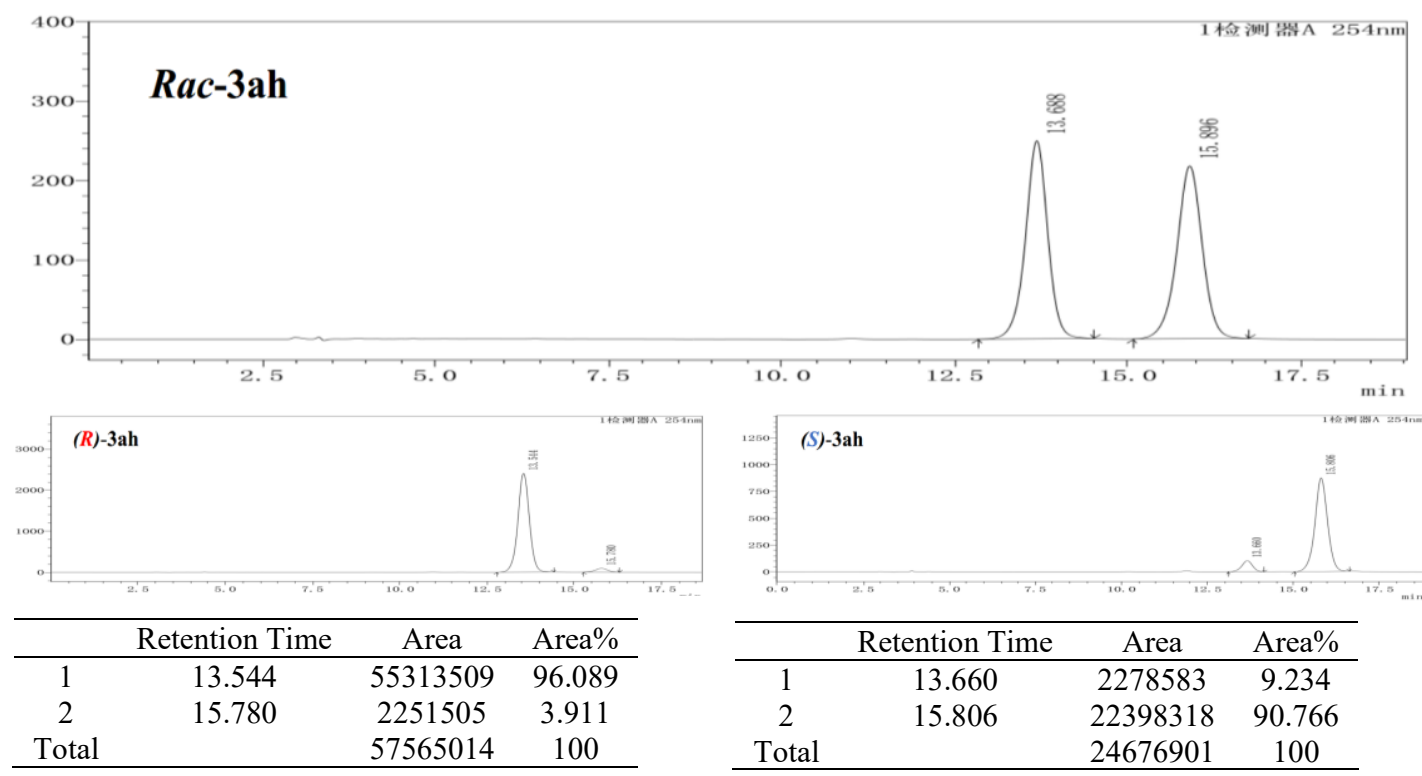


\section{1-benzyl-3-((4-chlorophenyl) ethynyl)-3-hydroxyindolin-2-one (3ai):}

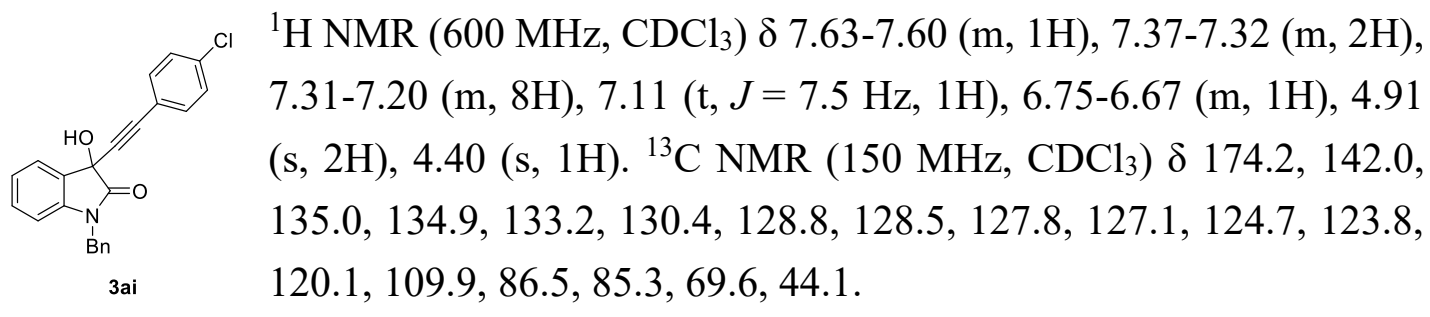

(R)-3ai, white solid, $34.3 \mathrm{mg}, 92 \%$ yield (dichloromethane/ethyl acetate $=50: 1$ ), 95\% $e e,[\alpha]_{\mathrm{D}}^{26}=-14.1\left(\mathrm{c}=0.43, \mathrm{CH}_{2} \mathrm{Cl}_{2}\right)$; HPLC: Daicel Chiralpak IA, n-hexane/2-propanol $=85 / 15$, flow rate $=1.0 \mathrm{~mL} / \mathrm{min}, \lambda=254 \mathrm{~nm}$, retention time: $14.2 \mathrm{~min}$ (major), 17.9 $\min$ (minor);

(S)-3ai, white solid, $33.6 \mathrm{mg}, 90 \%$ yield (dichloromethane/ethyl acetate $=50: 1$ ), 90\% $e e,[\alpha]_{\mathrm{D}}^{26}=+13.3\left(\mathrm{c}=0.38, \mathrm{CH}_{2} \mathrm{Cl}_{2}\right)\left(\operatorname{ref} .1:(S), 90 \% e e,[\alpha]_{\mathrm{D}}^{20}=+13.3(\mathrm{c}=0.54\right.$ in $\left.\mathrm{CH}_{2} \mathrm{Cl}_{2}\right)$ ); HPLC: Daicel Chiralpak IA, n-hexane/2-propanol $=85 / 15$, flow rate $=1.0$ $\mathrm{mL} / \mathrm{min}, \lambda=254 \mathrm{~nm}$, retention time: $14.1 \mathrm{~min}$ (minor), $17.6 \mathrm{~min}$ (major).

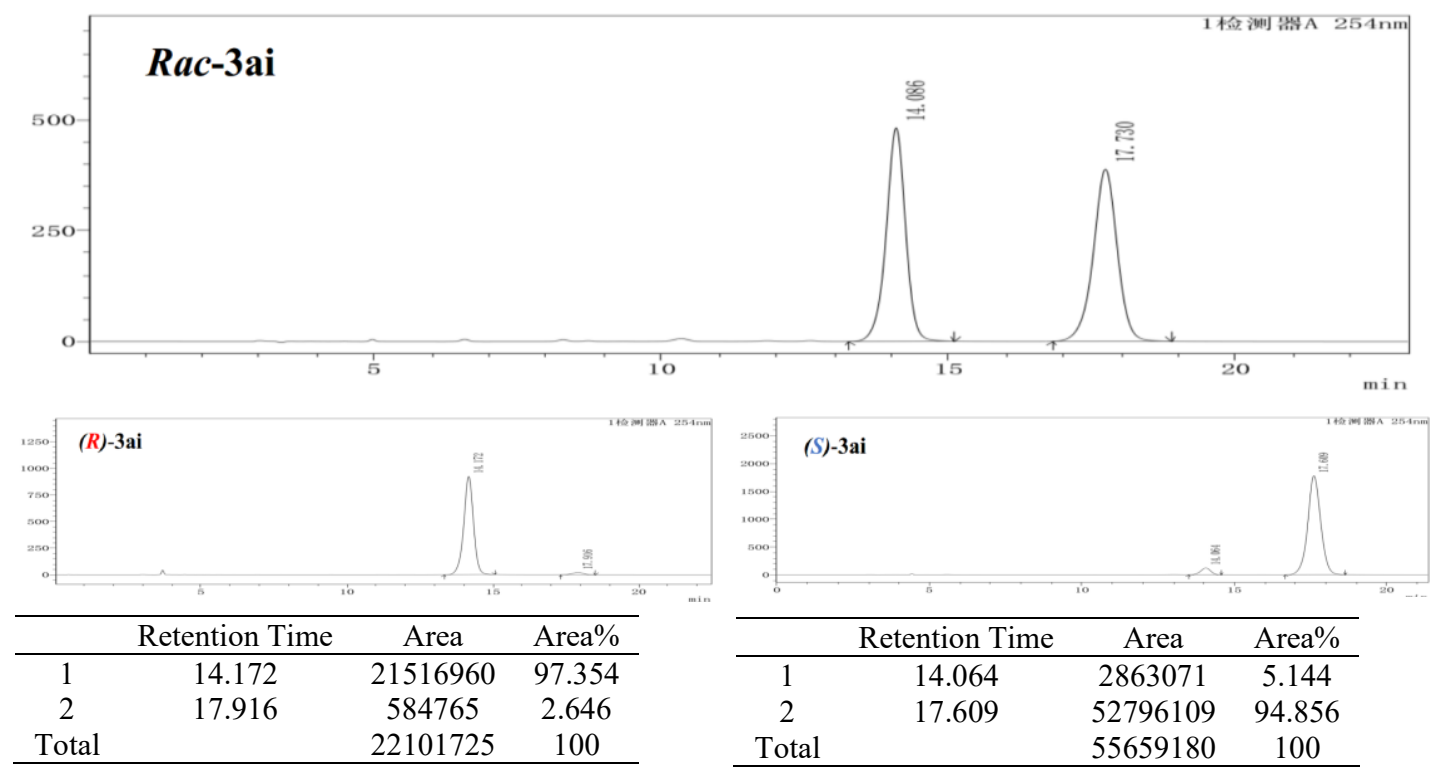


4-((1-benzyl-3-hydroxy-2-oxoindolin-3-yl) ethynyl) benzonitrile (3aj):

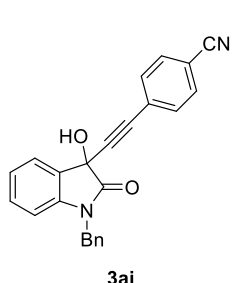

${ }^{1} \mathrm{H}$ NMR $\left(600 \mathrm{MHz}, \mathrm{CDCl}_{3}\right) \delta 7.60(\mathrm{~d}, J=7.3 \mathrm{~Hz}, 1 \mathrm{H}), 7.55(\mathrm{~d}, J=$ $8.3 \mathrm{~Hz}, 2 \mathrm{H}), 7.50(\mathrm{~d}, J=8.4 \mathrm{~Hz}, 2 \mathrm{H}), 7.33-7.26(\mathrm{~m}, 6 \mathrm{H}), 7.13(\mathrm{t}, J=$ $7.5 \mathrm{~Hz}, 1 \mathrm{H}), 6.75(\mathrm{~d}, J=7.9 \mathrm{~Hz}, 1 \mathrm{H}), 4.93(\mathrm{~s}, 2 \mathrm{H}), 4.14(\mathrm{~s}, 1 \mathrm{H}) .{ }^{13} \mathrm{C}$ NMR $\left(150 \mathrm{MHz}, \mathrm{CDCl}_{3}\right) \delta 173.7,142.1,134.8,132.5,131.9,130.7$, $128.9,128.3,127.9,127.1,126.4,124.8,123.9,118.1,112.4,110.1$,

89.7, 84.5, 69.5, 44.2. HRMS (ESI) $\mathrm{m} / z$ : $[\mathrm{M}+\mathrm{H}]^{+}$Calcd for $\mathrm{C}_{24} \mathrm{H}_{17} \mathrm{~N}_{2} \mathrm{O}_{2}{ }^{+}$365.1285; Found: 365.1273 .

(R)-3aj, white solid, $20.4 \mathrm{mg}, 56 \%$ yield (dichloromethane/ethyl acetate $=30: 1$ ), 63\% $e e,[\alpha]_{\mathrm{D}}^{26}=-5.5\left(\mathrm{c}=0.26, \mathrm{CH}_{2} \mathrm{Cl}_{2}\right)$; HPLC: Daicel Chiralpak IA, n-hexane/2-propanol = $85 / 15$, flow rate $=1.0 \mathrm{~mL} / \mathrm{min}, \lambda=254 \mathrm{~nm}$, retention time: $26.4 \mathrm{~min}$ (major), $34.3 \mathrm{~min}$ (minor);

(S)-3aj, white solid, $22.6 \mathrm{mg}, 62 \%$ yield (dichloromethane/ethyl acetate $=30: 1$ ), 97\% ee. $[\alpha]_{\mathrm{D}}^{26}=+9.9\left(\mathrm{c}=0.61, \mathrm{CH}_{2} \mathrm{Cl}_{2}\right)$; HPLC: Daicel Chiralpak IA, n-hexane/2-propanol $=85 / 15$, flow rate $=1.0 \mathrm{~mL} / \mathrm{min}, \lambda=254 \mathrm{~nm}$, retention time: $26.5 \mathrm{~min}$ (minor), 34.1 min (major).

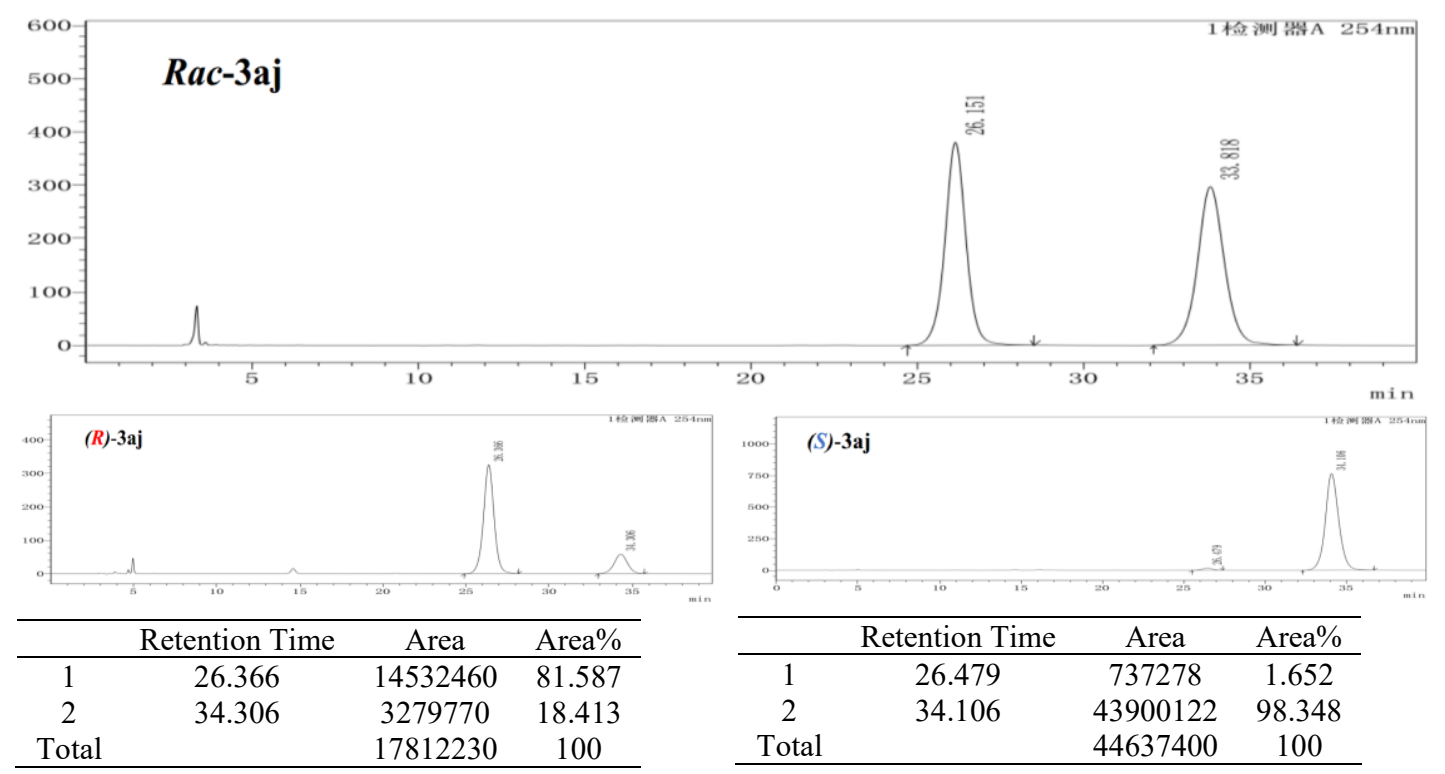




\section{1-benzyl-3-((4-fluorophenyl) ethynyl)-3-hydroxyindolin-2-one (3ak):}

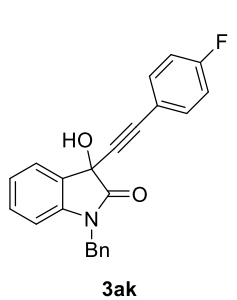

${ }^{1} \mathrm{H}$ NMR $\left(600 \mathrm{MHz}, \mathrm{CDCl}_{3}\right) \delta 7.61(\mathrm{~d}, J=7.4 \mathrm{~Hz}, 1 \mathrm{H}), 7.45-7.38(\mathrm{~m}$, $2 \mathrm{H}), 7.31-7.23(\mathrm{~m}, 6 \mathrm{H}), 7.11(\mathrm{t}, J=7.5 \mathrm{~Hz}, 1 \mathrm{H}), 6.95(\mathrm{t}, J=8.7 \mathrm{~Hz}$, $2 \mathrm{H}), 6.72(\mathrm{~d}, J=7.8 \mathrm{~Hz}, 1 \mathrm{H}), 4.95-4.89(\mathrm{~m}, 2 \mathrm{H}), 4.12(\mathrm{~s}, 1 \mathrm{H}) .{ }^{13} \mathrm{C}$ NMR $\left(150 \mathrm{MHz}, \mathrm{CDCl}_{3}\right) \delta 174.1,163.7,162.0,142.1,134.9,134.0$ $(\mathrm{d}, J=8.5 \mathrm{~Hz}), 130.4,128.8,127.8,127.1,124.7,123.8,117.7,115.6$, $115.4,109.9,85.4,85.3,69.6,44.1$.

$(R)-3 a k$, white solid, $32.1 \mathrm{mg}, 90 \%$ yield (dichloromethane/ethyl acetate $=50: 1$ ), 92\% $e e,[\alpha]_{\mathrm{D}}^{26}=-16.8\left(\mathrm{c}=0.60, \mathrm{CH}_{2} \mathrm{Cl}_{2}\right)$; HPLC: Daicel Chiralpak IA, n-hexane/2-propanol $=85 / 15$, flow rate $=1.0 \mathrm{~mL} / \mathrm{min}, \lambda=254 \mathrm{~nm}$, retention time: $13.6 \mathrm{~min}$ (major), 17.7 $\min$ (minor);

(S)-3ak, white solid, $34.6 \mathrm{mg}, 97 \%$ yield (dichloromethane/ethyl acetate $=50: 1$ ), 85\% $e e,[\alpha]_{\mathrm{D}}^{26}=+14.5\left(\mathrm{c}=1.11, \mathrm{CH}_{2} \mathrm{Cl}_{2}\right)\left(\operatorname{ref} .1:(S), 92 \% e e,[\alpha]_{\mathrm{D}}^{20}=+15.6(\mathrm{c}=0.54\right.$ in $\mathrm{CH}_{2} \mathrm{Cl}_{2}$ )); HPLC: Daicel Chiralpak IA, n-hexane/2-propanol $=85 / 15$, flow rate $=1.0$ $\mathrm{mL} / \mathrm{min}, \lambda=254 \mathrm{~nm}$, retention time: $13.3 \mathrm{~min}$ (minor), $17.0 \mathrm{~min}$ (major).
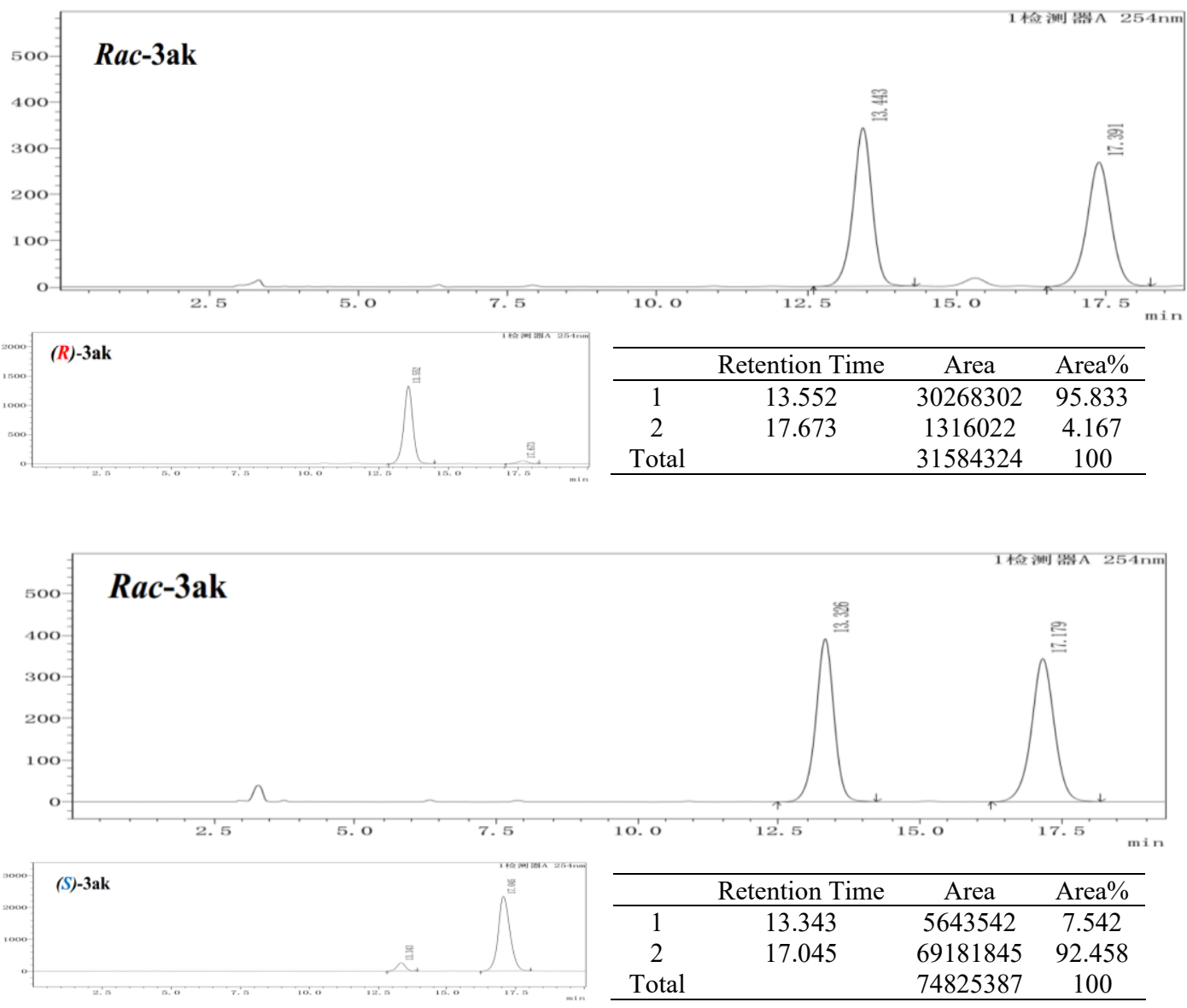


\section{1-benzyl-3-((4-ethylphenyl) ethynyl)-3-hydroxyindolin-2-one (3al):}

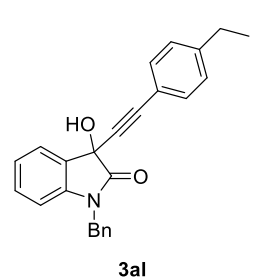

${ }^{1} \mathrm{H} \mathrm{NMR}\left(600 \mathrm{MHz}, \mathrm{CDCl}_{3}\right) \delta$ 7.62-7.60 (m, $\left.1 \mathrm{H}\right), 7.36(\mathrm{~d}, J=8.0 \mathrm{~Hz}$, $2 \mathrm{H}), 7.31-7.29(\mathrm{~m}, 4 \mathrm{H}), 7.26-7.22(\mathrm{~m}, 2 \mathrm{H}), 7.12-7.09(\mathrm{~m}, 3 \mathrm{H}), 6.71-$ $6.70(\mathrm{~m}, 1 \mathrm{H}), 4.92(\mathrm{~s}, 2 \mathrm{H}), 3.82(\mathrm{~s}, 1 \mathrm{H}), 2.62(\mathrm{q}, \mathrm{J}=7.6 \mathrm{~Hz}, 2 \mathrm{H}), 1.20$ $(\mathrm{t}, J=7.6 \mathrm{~Hz}, 3 \mathrm{H}) .{ }^{13} \mathrm{C}$ NMR $\left(150 \mathrm{MHz}, \mathrm{CDCl}_{3}\right) \delta 174.1,145.5$, $142.1,135.0,132.0,130.3,128.8,127.7,127.1,124.7,123.7,118.7$, $109.8,86.8,84.7,69.6,44.0,28.8,15.2$. HRMS (ESI) $m / z:[\mathrm{M}+\mathrm{H}]^{+}$Calcd for $\mathrm{C}_{25} \mathrm{H}_{22} \mathrm{NO}_{2}{ }^{+}$368.1645; Found: 368.1647 .

(R)-3al, white solid, $33.8 \mathrm{mg}$, 92\% yield (dichloromethane/ethyl acetate $=40: 1$ ), 93\% $e e,[\alpha]_{\mathrm{D}}^{26}=-19.6\left(\mathrm{c}=0.77, \mathrm{CH}_{2} \mathrm{Cl}_{2}\right)$; HPLC: Daicel Chiralpak AD-H, n-hexane/2propanol $=85 / 15$, flow rate $=1.0 \mathrm{~mL} / \mathrm{min}, \lambda=254 \mathrm{~nm}$, retention time: $19.1 \mathrm{~min}$ (major), $21.5 \mathrm{~min}$ (minor);

(S)-3al, white solid, $15.8 \mathrm{mg}$, 43\% yield (dichloromethane/ethyl acetate $=40: 1$ ), 55\% $e e,[\alpha]_{\mathrm{D}}^{26}=+4.0\left(\mathrm{c}=0.50, \mathrm{CH}_{2} \mathrm{Cl}_{2}\right)$; HPLC: Daicel Chiralpak AD-H, n-hexane/2propanol $=85 / 15$, flow rate $=1.0 \mathrm{~mL} / \mathrm{min}, \lambda=254 \mathrm{~nm}$, retention time: $19.5 \mathrm{~min}$ (minor), 22.1 min (major).

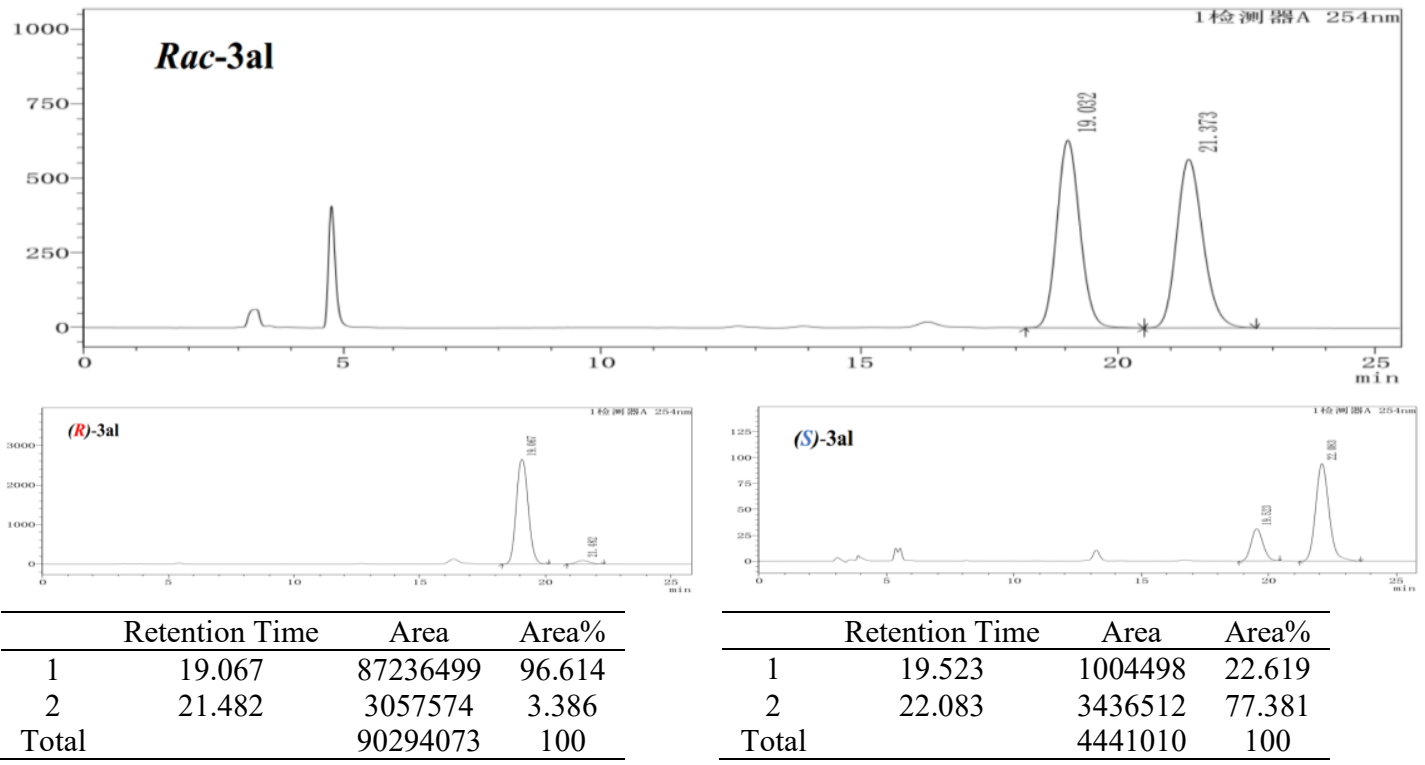




\section{1-benzyl-3-(cyclopropylethynyl)-3-hydroxyindolin-2-one (3am):}

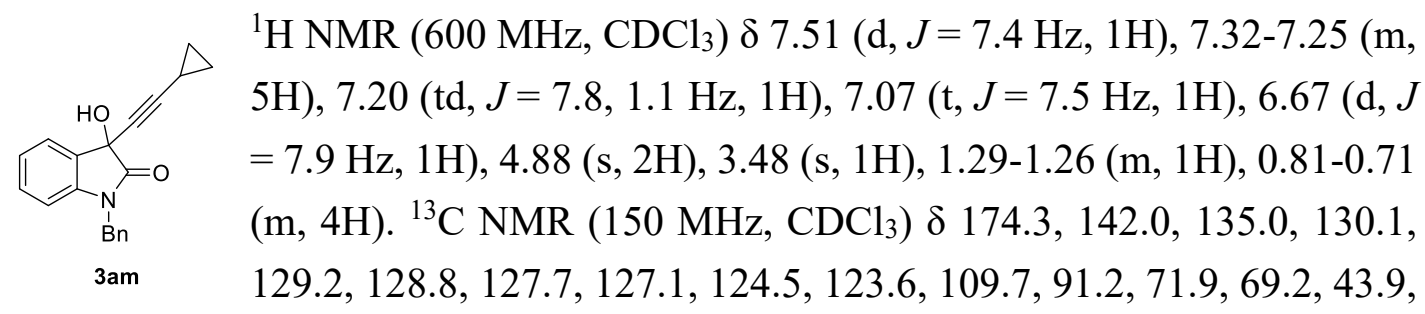

$8.5,-0.3$.

(R)-3am, white solid, $26.1 \mathrm{mg}, 86 \%$ yield (dichloromethane/ethyl acetate $=40: 1$ ), $82 \%$ $e e,[\alpha]_{\mathrm{D}}^{26}=-15.3\left(\mathrm{c}=1.31, \mathrm{CH}_{2} \mathrm{Cl}_{2}\right)$; HPLC: Daicel Chiralpak IA, n-hexane/2-propanol $=85 / 15$, flow rate $=1.0 \mathrm{~mL} / \mathrm{min}, \lambda=254 \mathrm{~nm}$, retention time: $10.3 \mathrm{~min}$ (major), 12.1 $\min$ (minor);

(S)-3am, white solid, $27.6 \mathrm{mg}$, 91\% yield (dichloromethane/ethyl acetate $=40: 1$ ), 61\% $e e,[\alpha]_{\mathrm{D}}^{26}=+8.3\left(\mathrm{c}=1.02, \mathrm{CH}_{2} \mathrm{Cl}_{2}\right)\left(\operatorname{ref} .1:(S), 92 \% e e,[\alpha]_{\mathrm{D}}^{20}=+17.1\left(\mathrm{c}=0.57\right.\right.$ in $\left.\left.\mathrm{CH}_{2} \mathrm{Cl}_{2}\right)\right)$; HPLC: Daicel Chiralpak IA, n-hexane/2-propanol $=85 / 15$, flow rate $=1.0 \mathrm{~mL} / \mathrm{min}, \lambda$ $=254 \mathrm{~nm}$, retention time: $10.4 \mathrm{~min}$ (minor), $12.0 \mathrm{~min}$ (major).

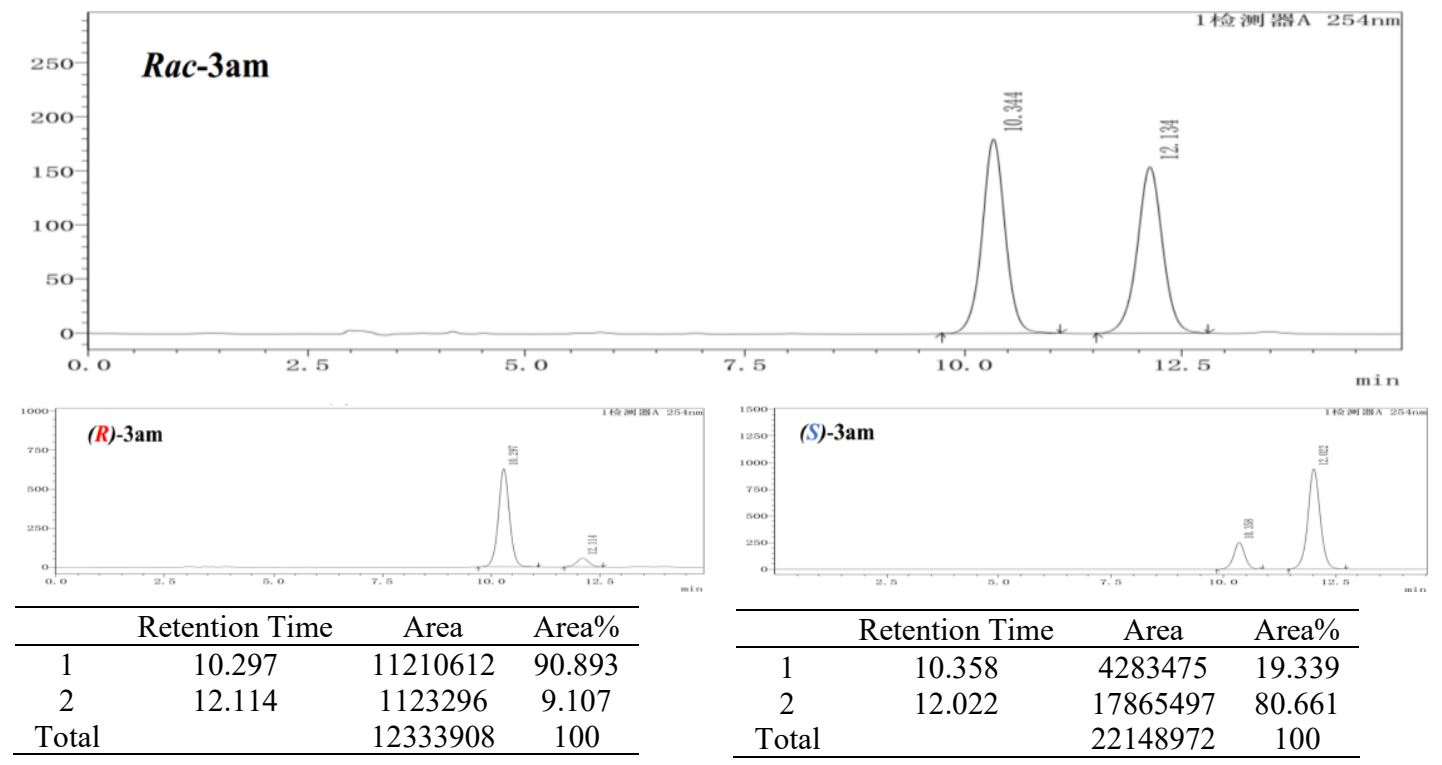




\section{Copies of NMR Spectra}

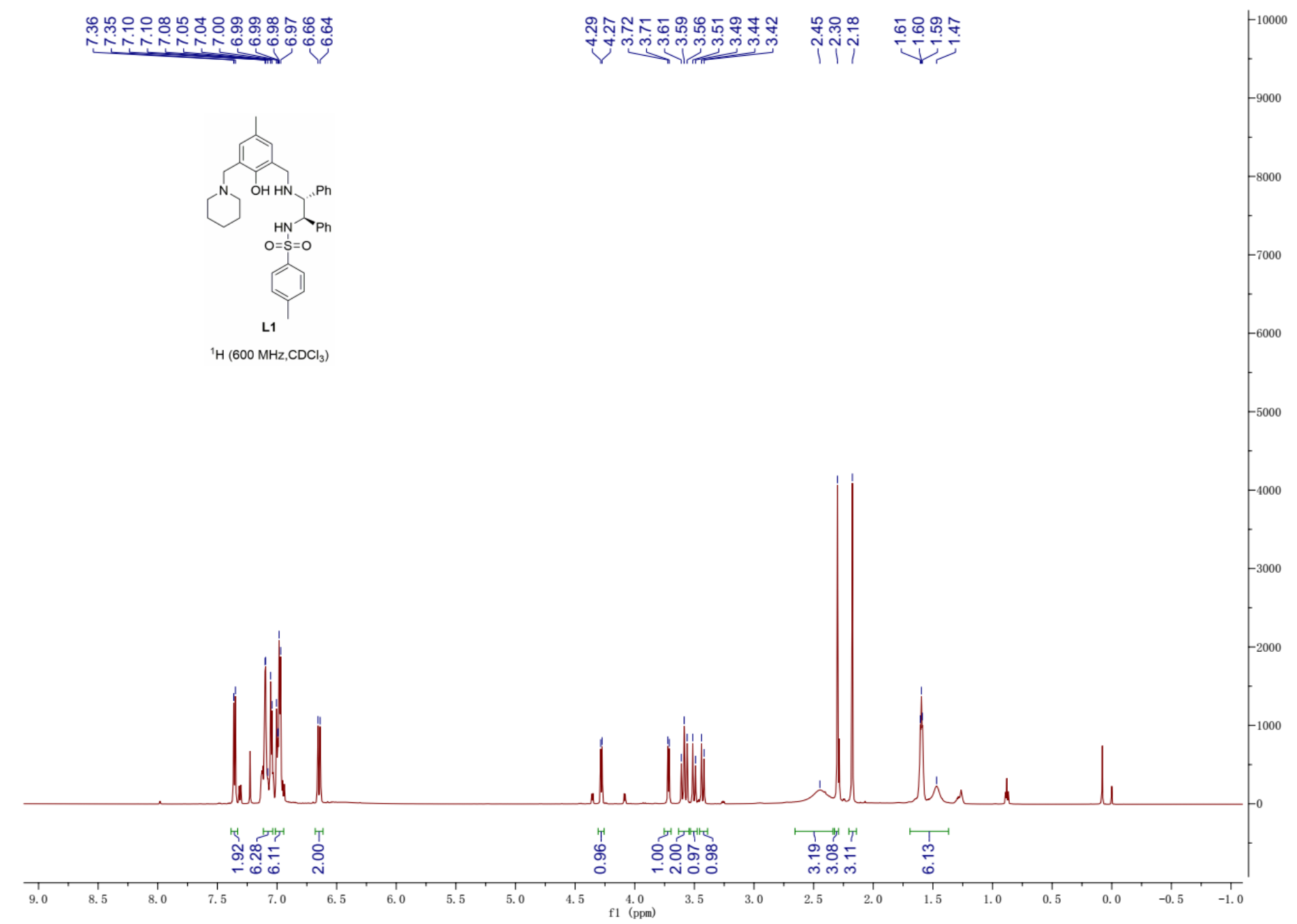




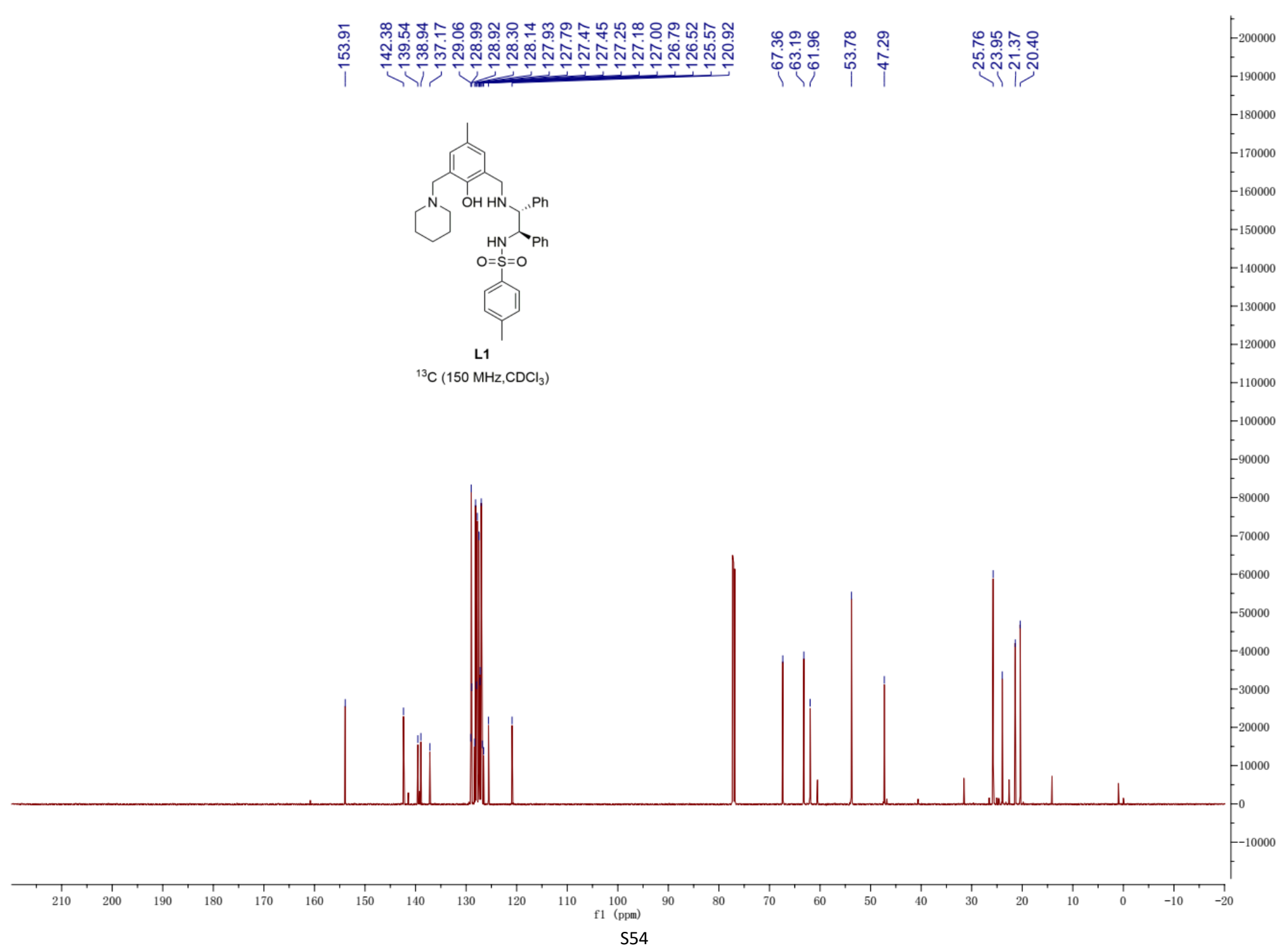




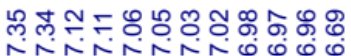

雨

N-NiñN000000

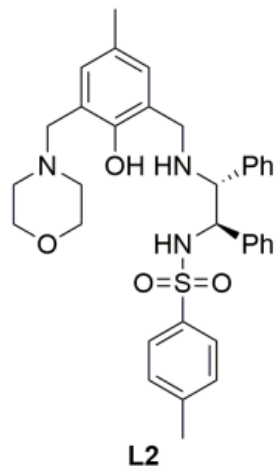

${ }^{1} \mathrm{H}\left(600 \mathrm{MHz}, \mathrm{CDCl}_{3}\right)$

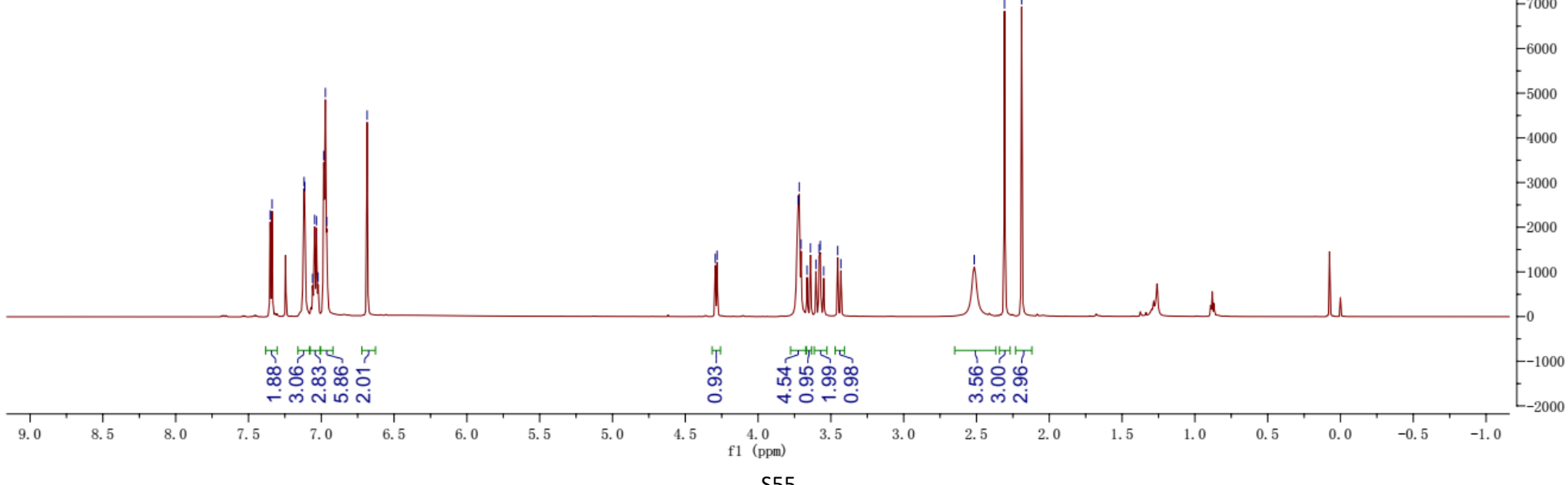

\$55 


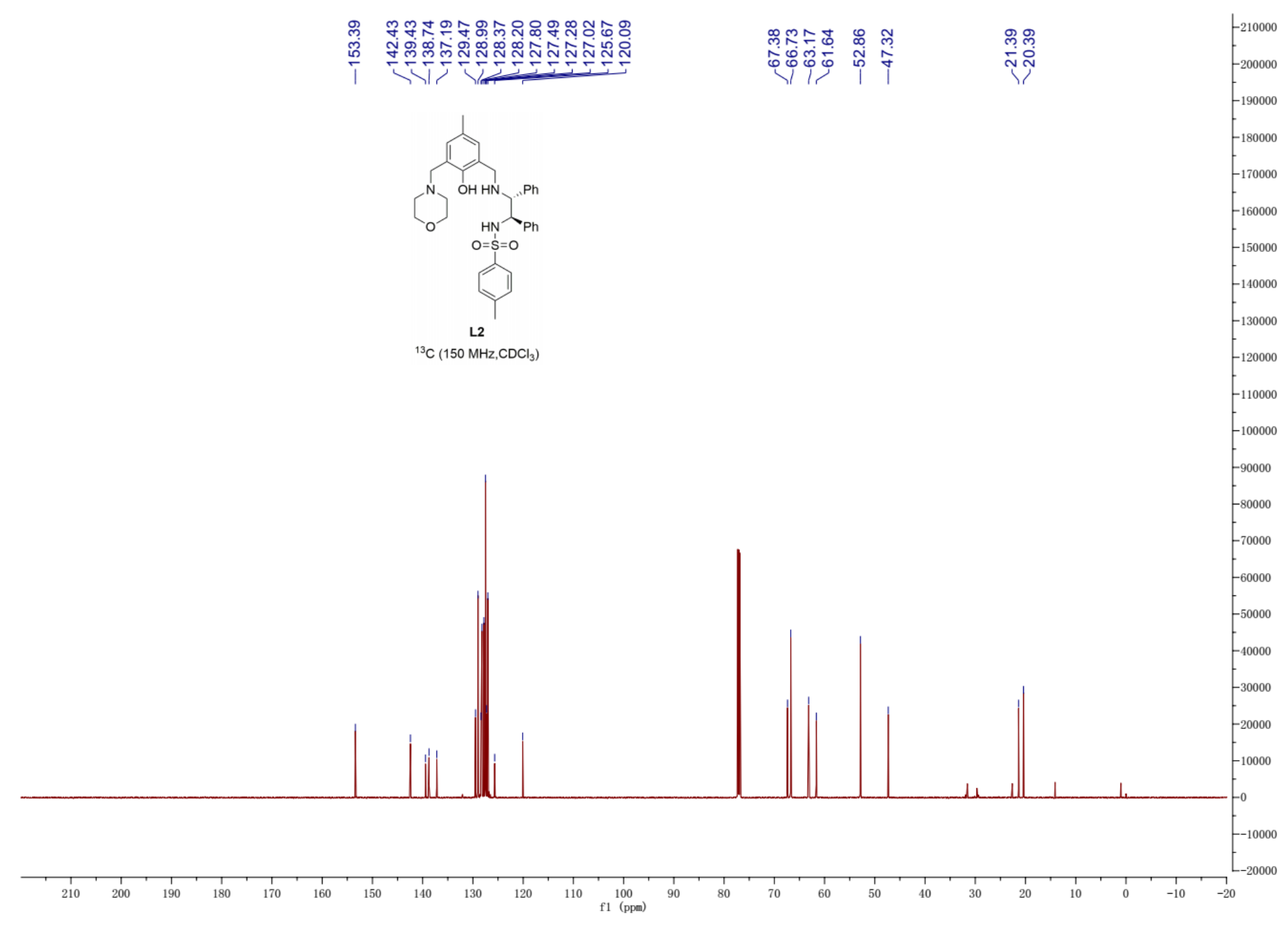




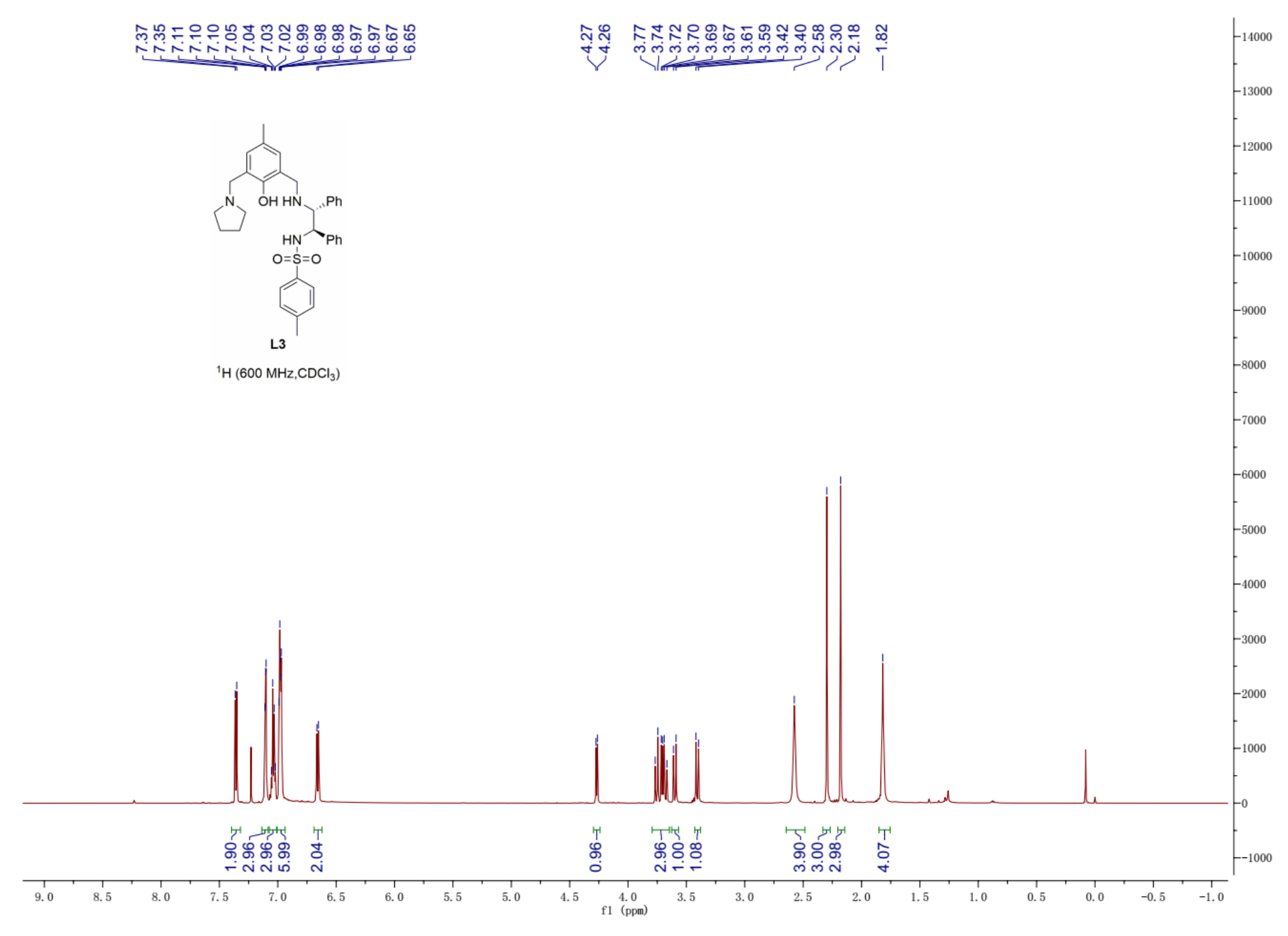

S57 


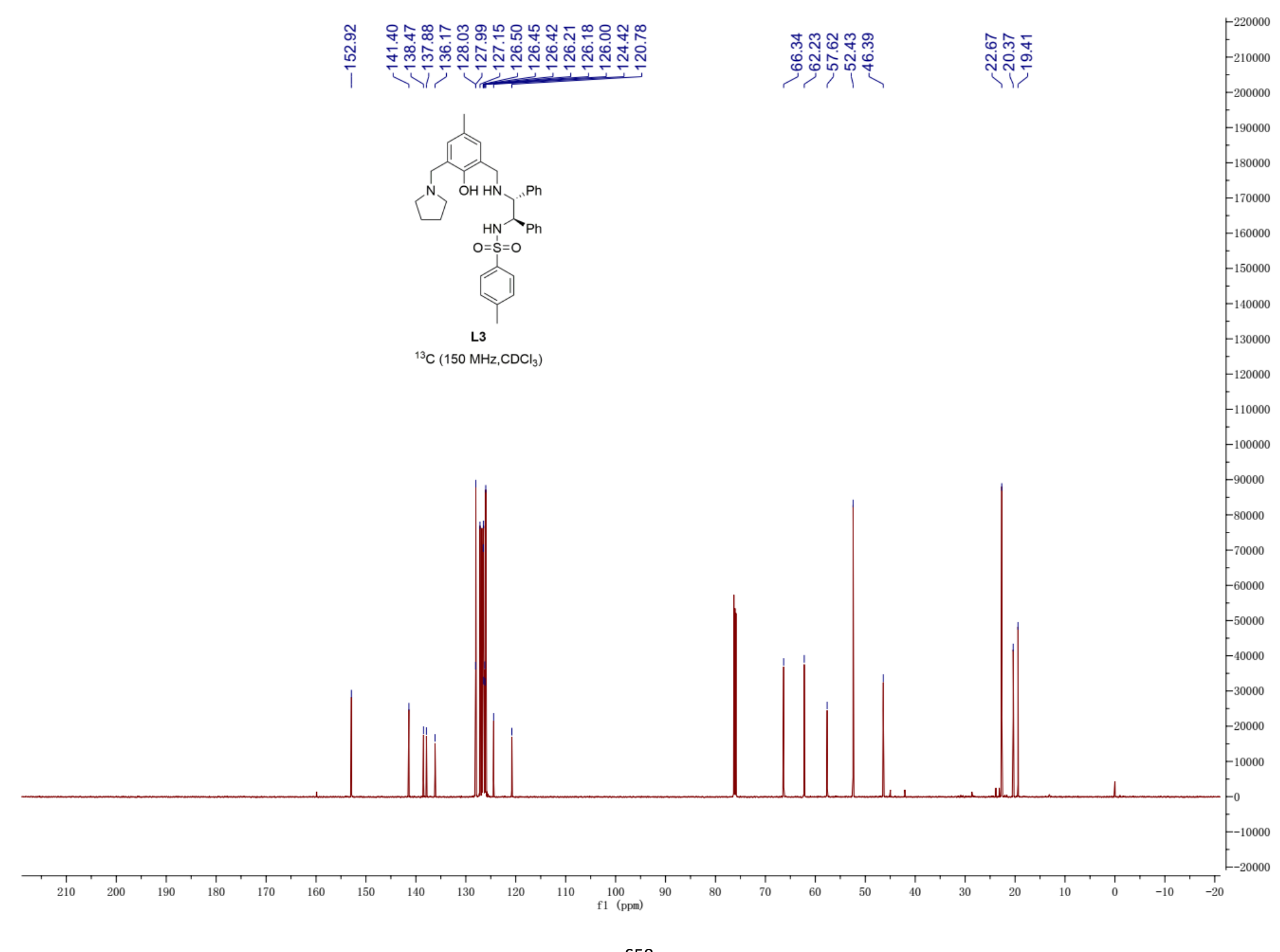




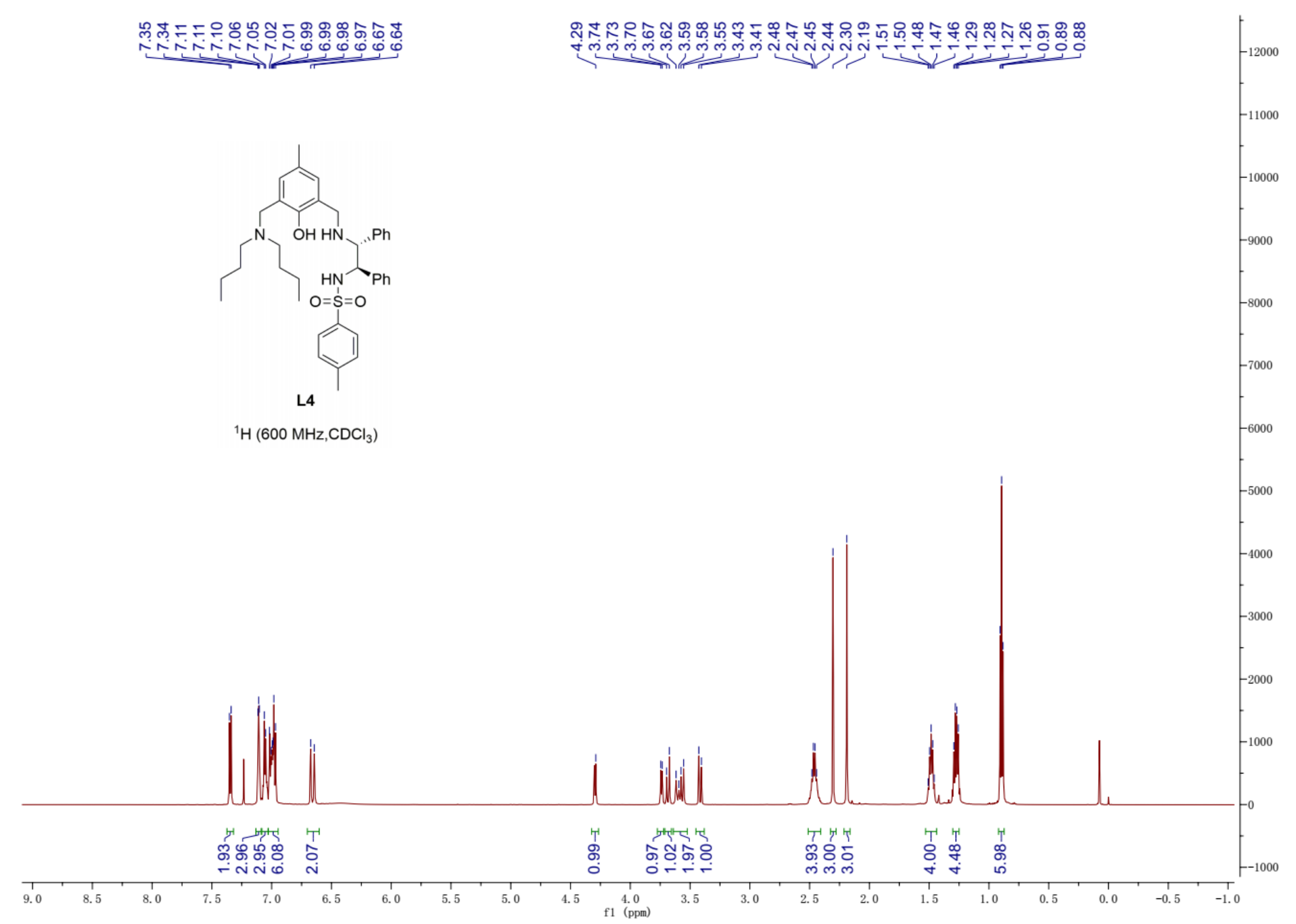




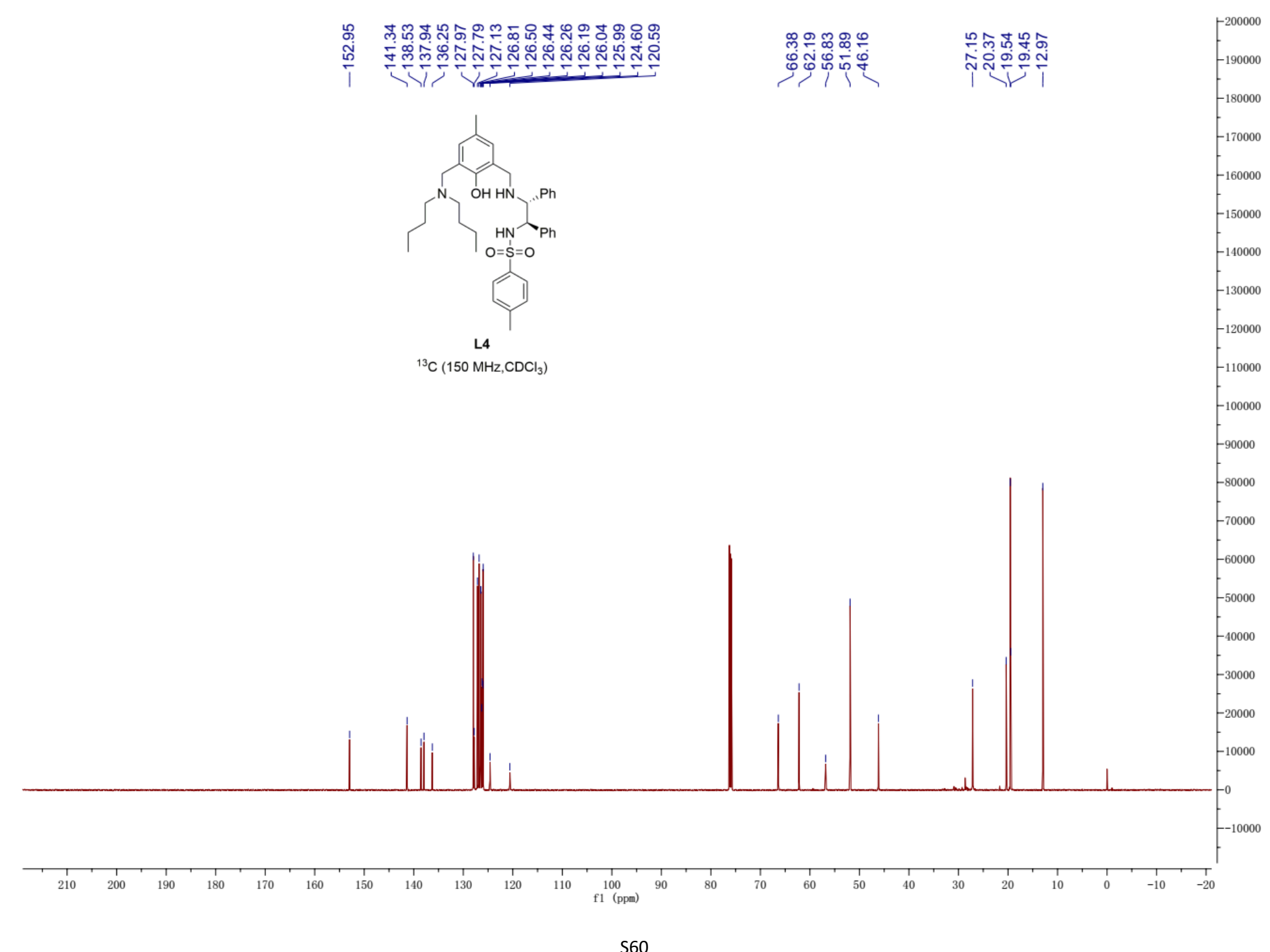




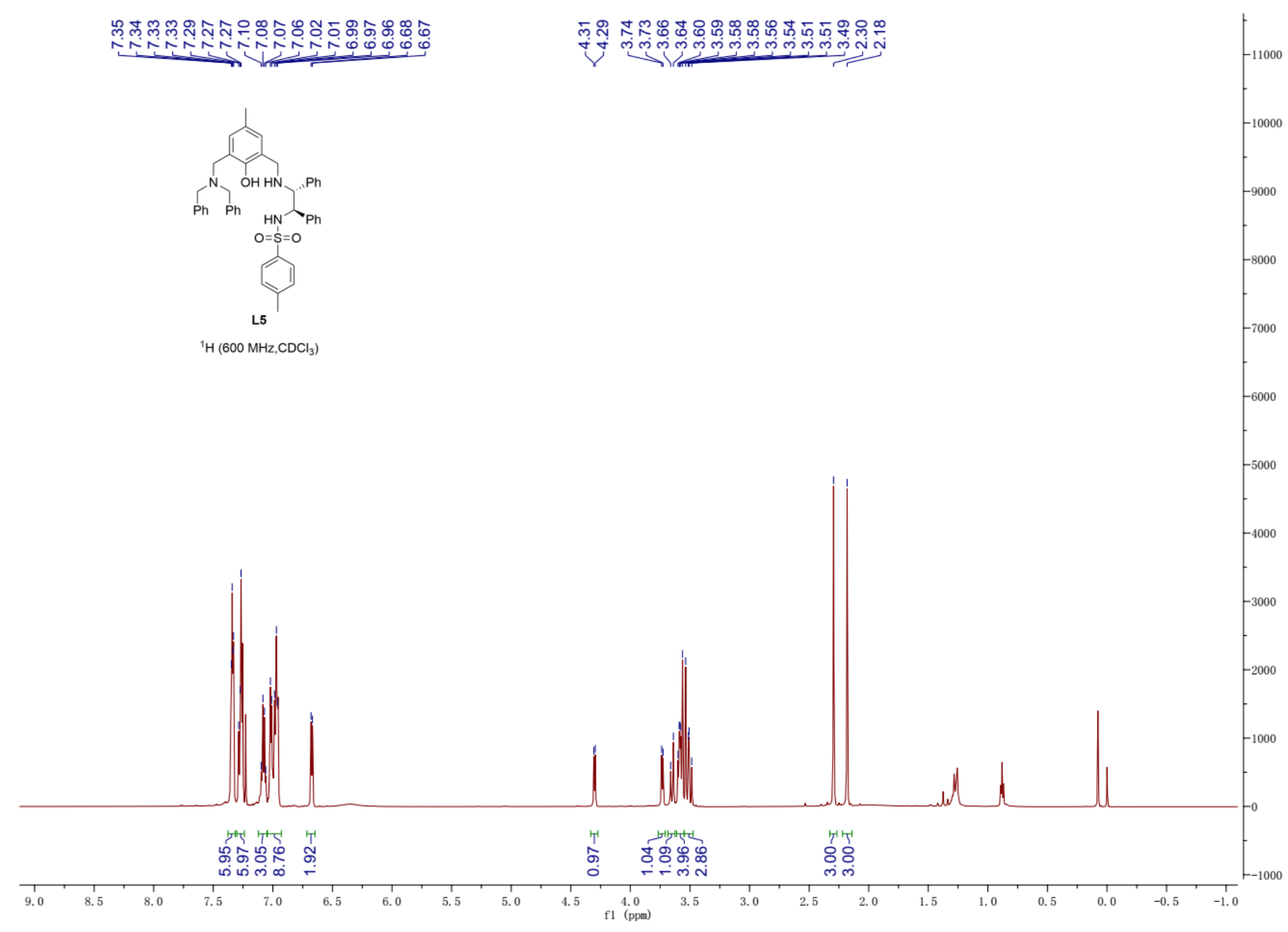




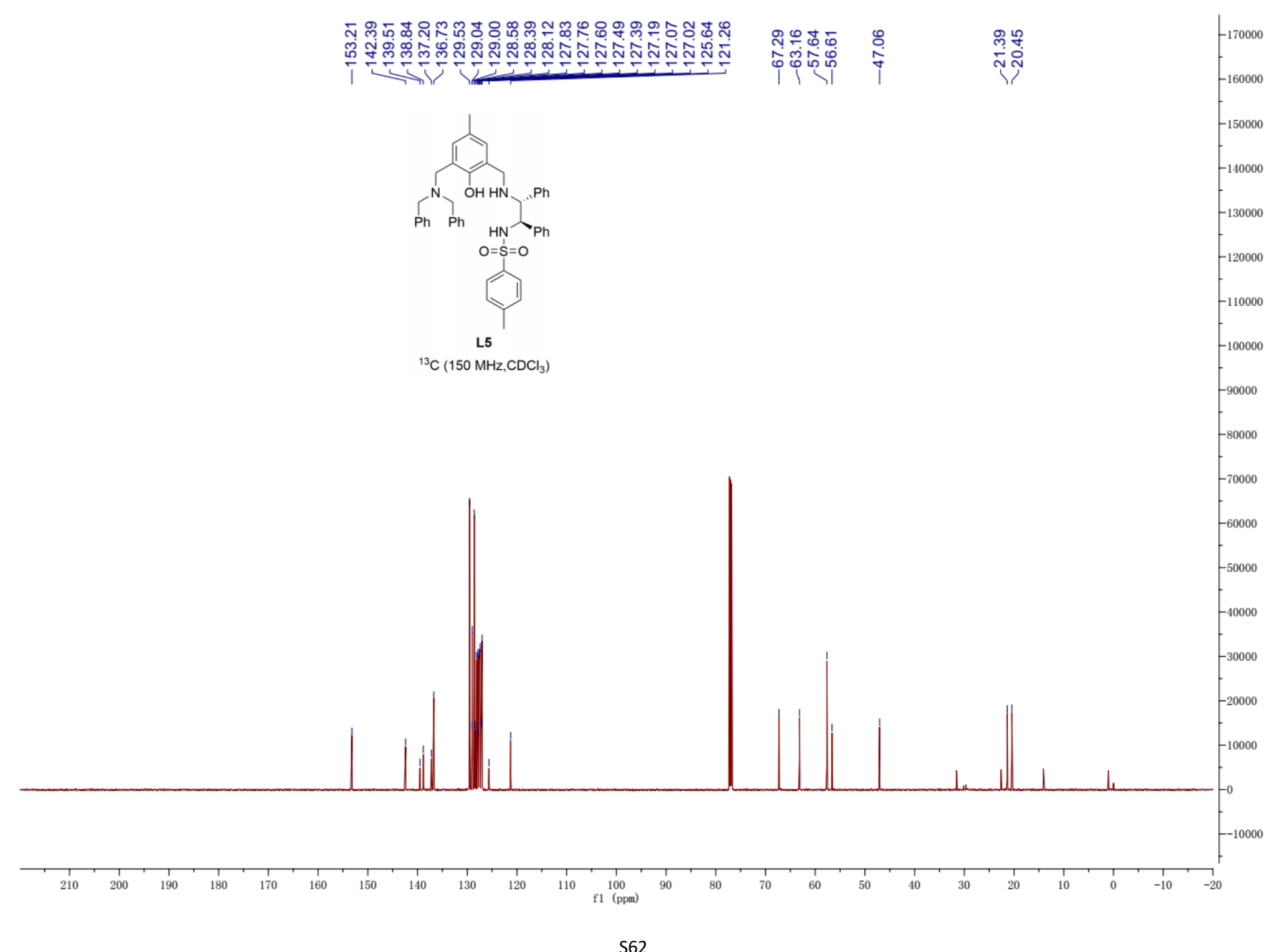

S62 


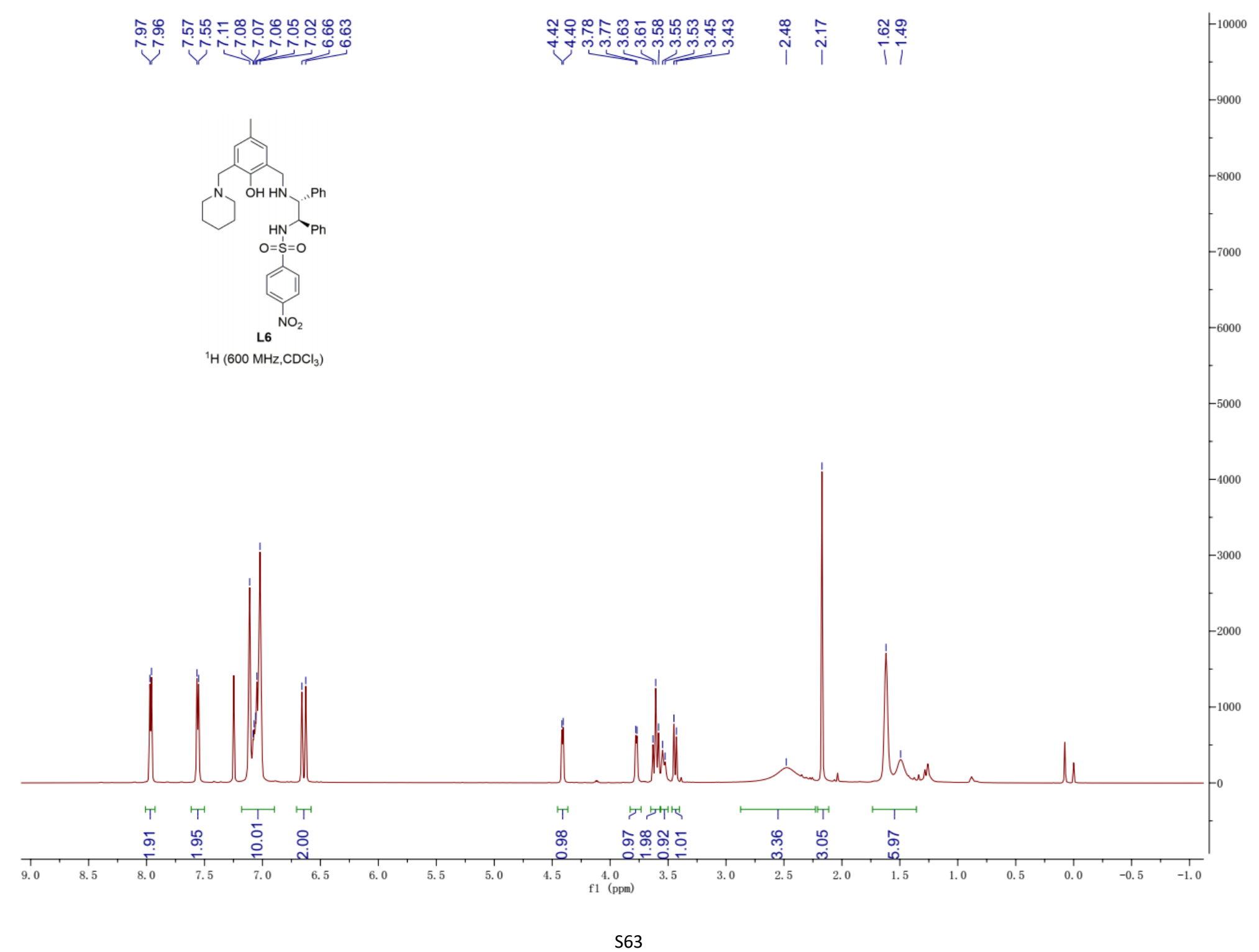




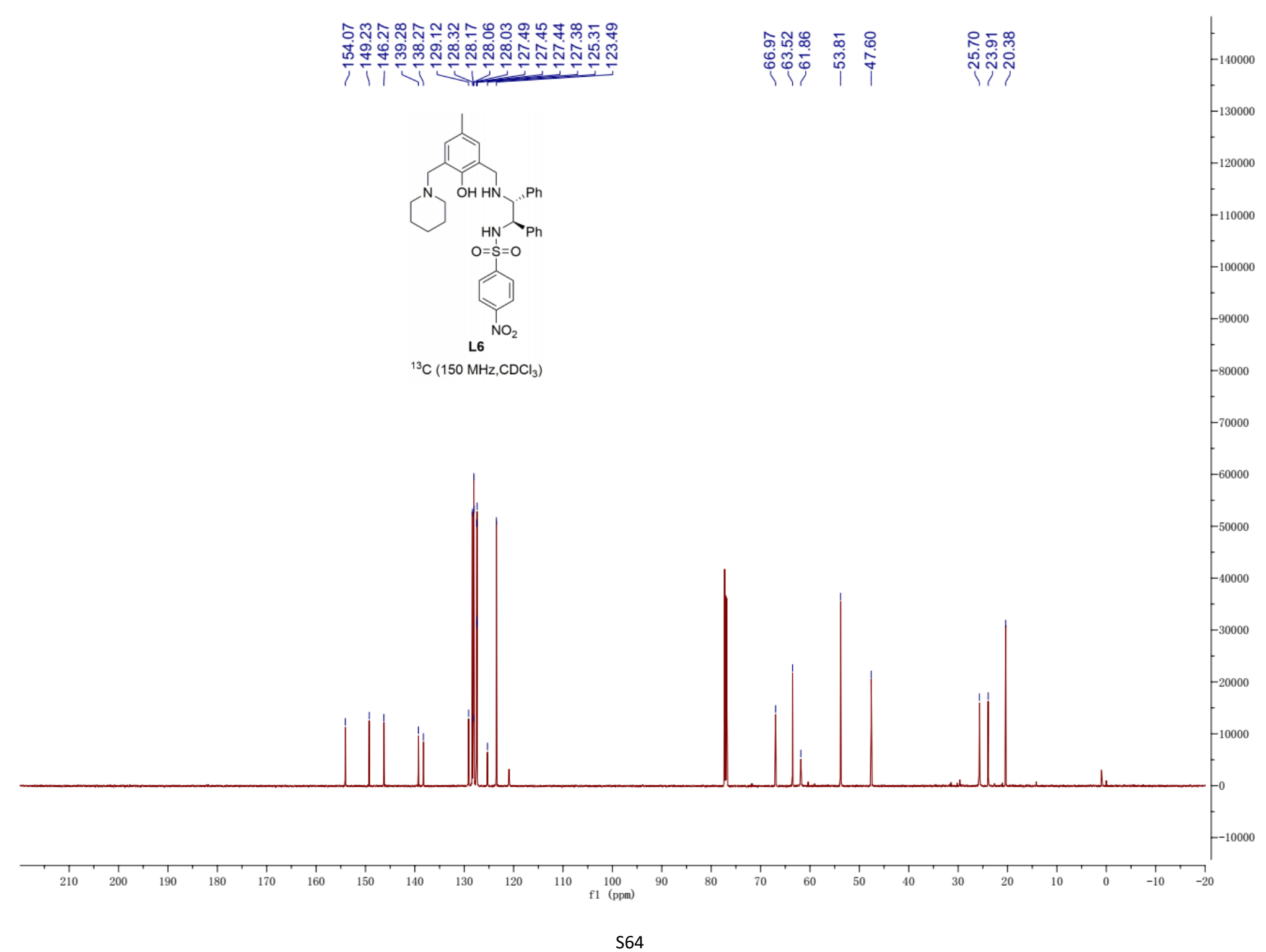




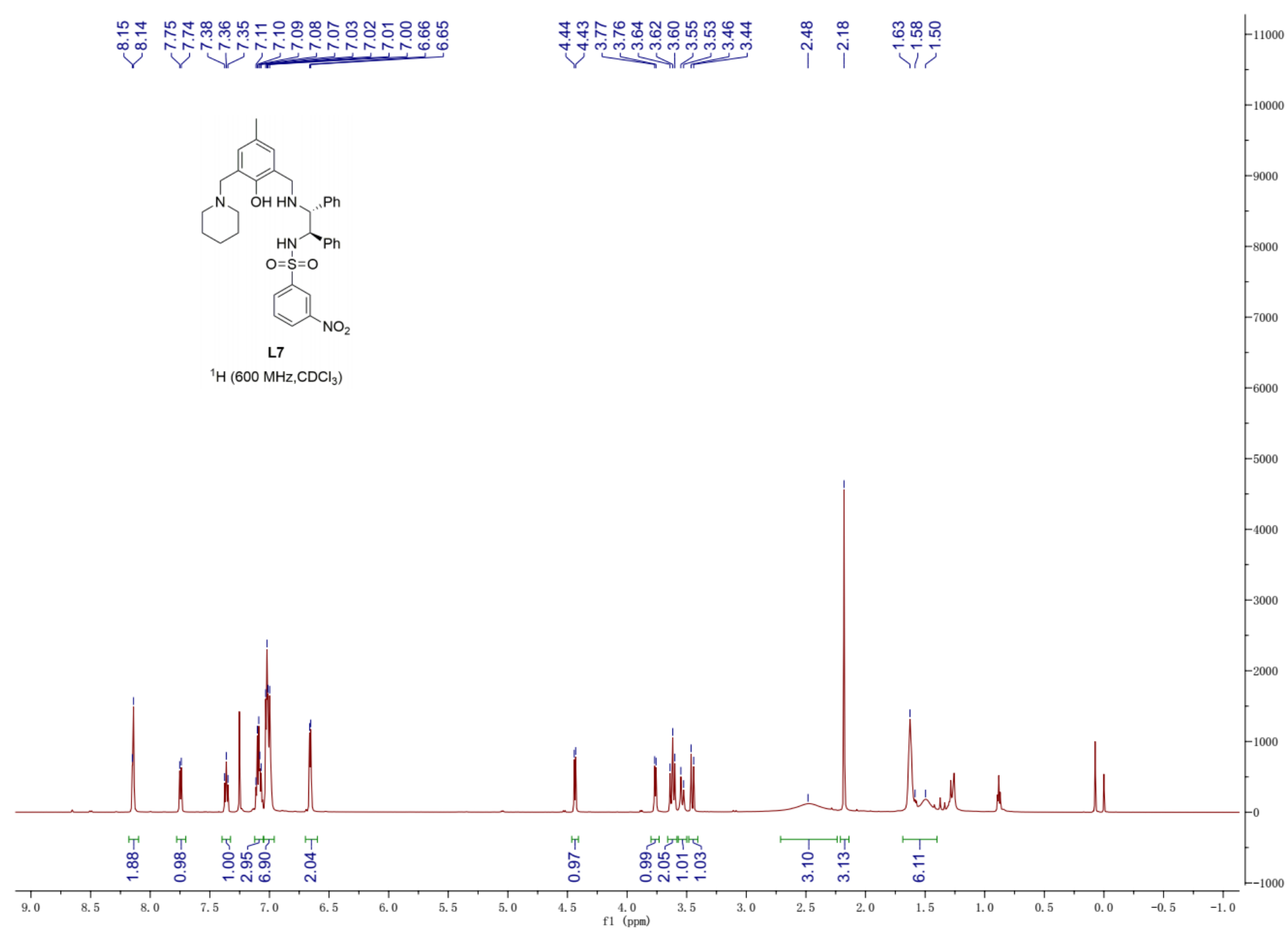




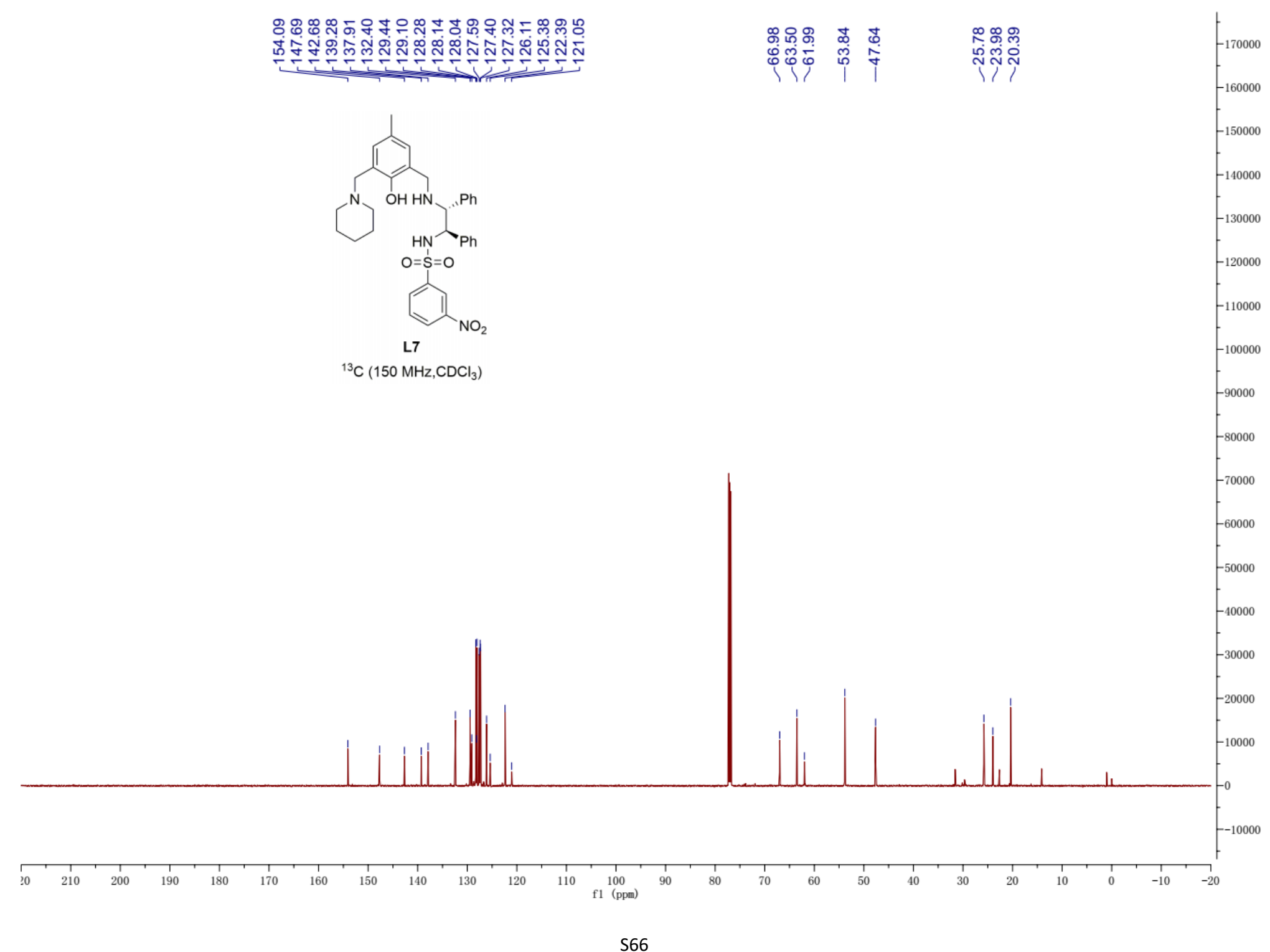




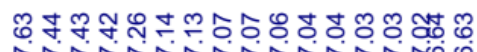

㒖

14000

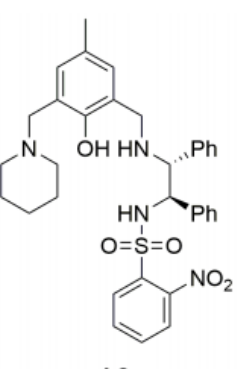

${ }^{1} \mathrm{H}\left(600 \mathrm{MHz}, \mathrm{CDCl}_{3}\right)$

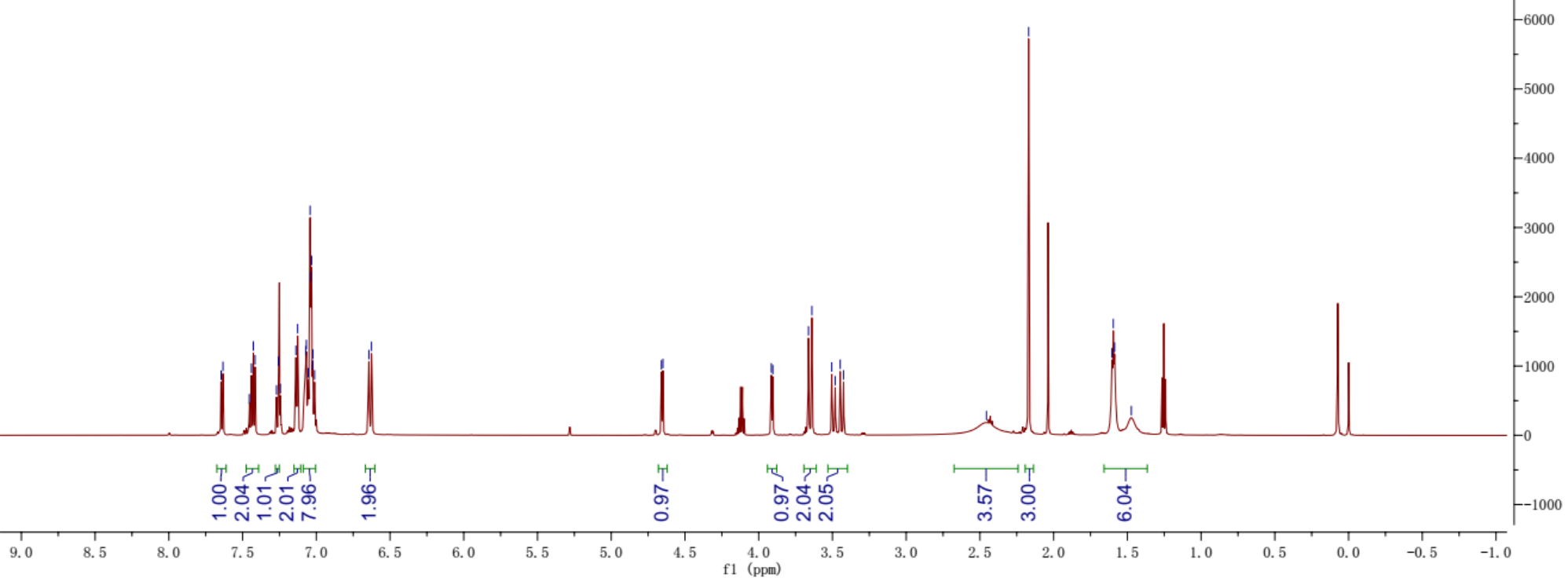




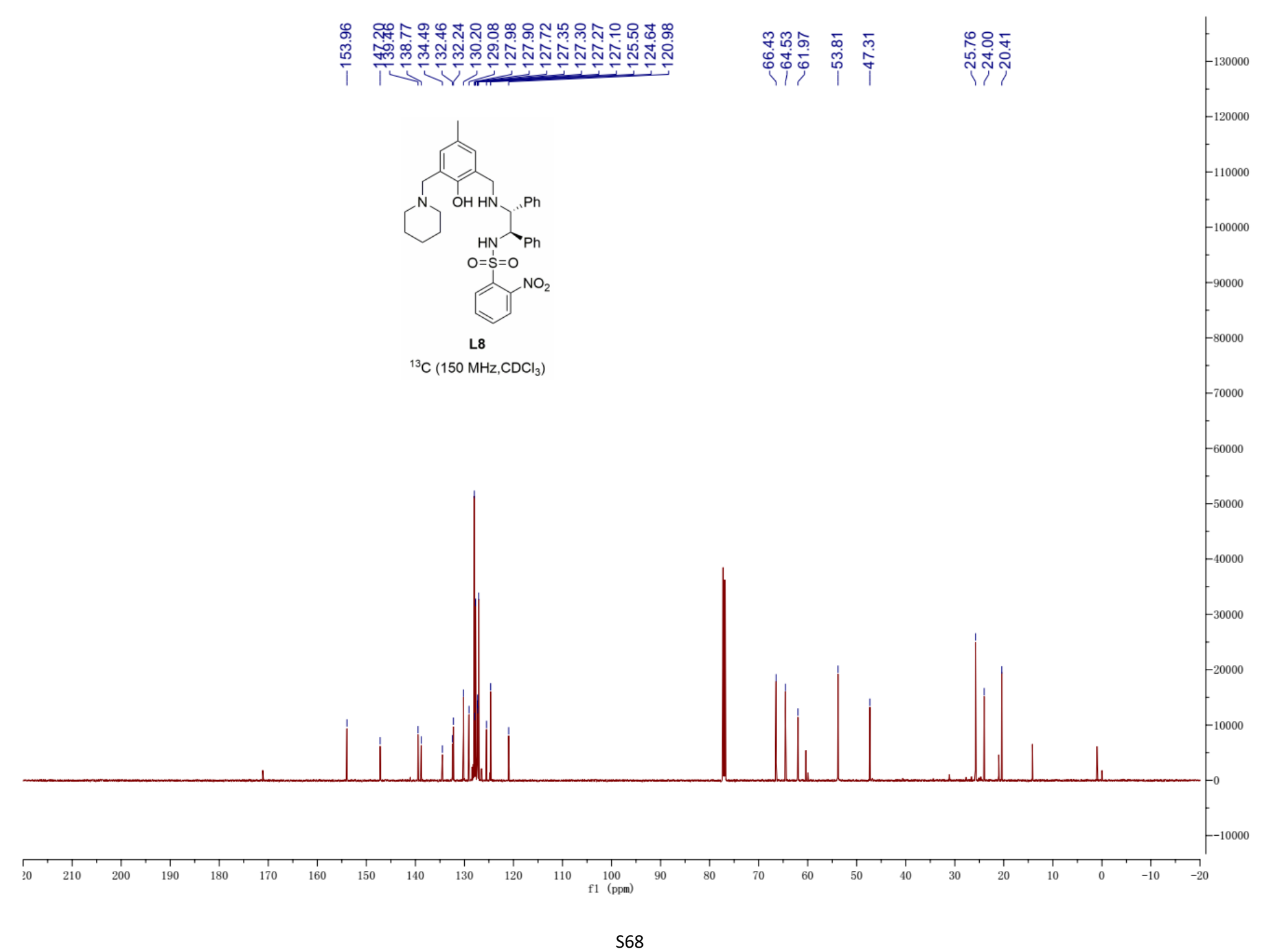




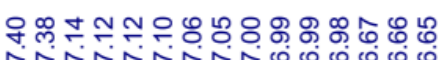

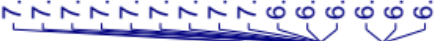

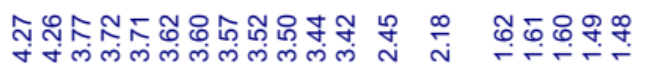

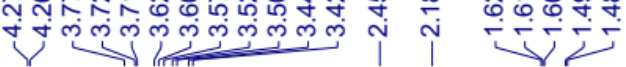

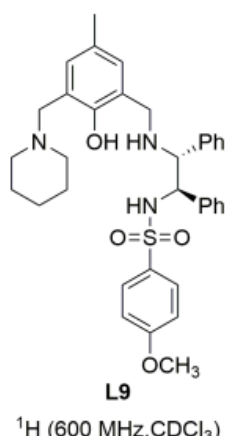

$\left.{ }^{1} \mathrm{H}\left(600 \mathrm{MHz}^{\mathrm{CDCl}}\right)_{3}\right)$

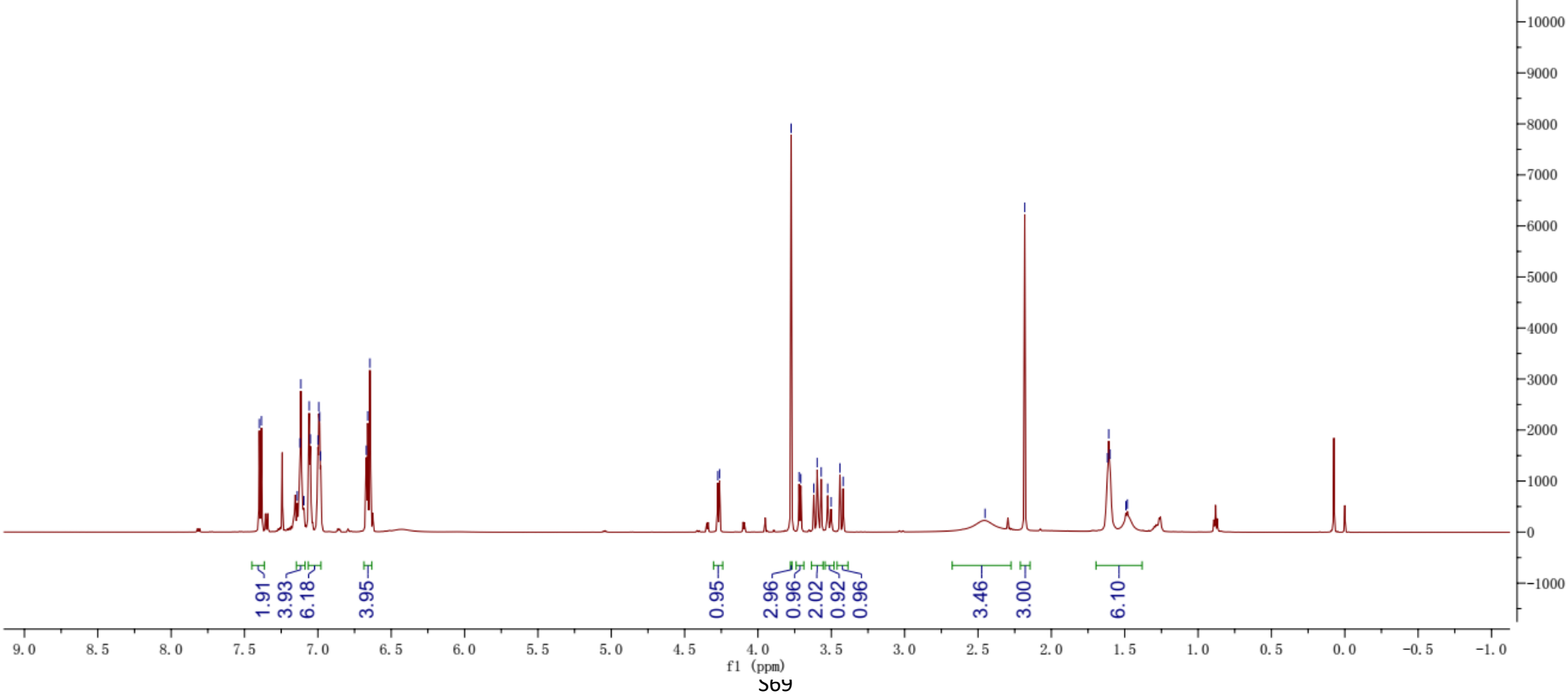




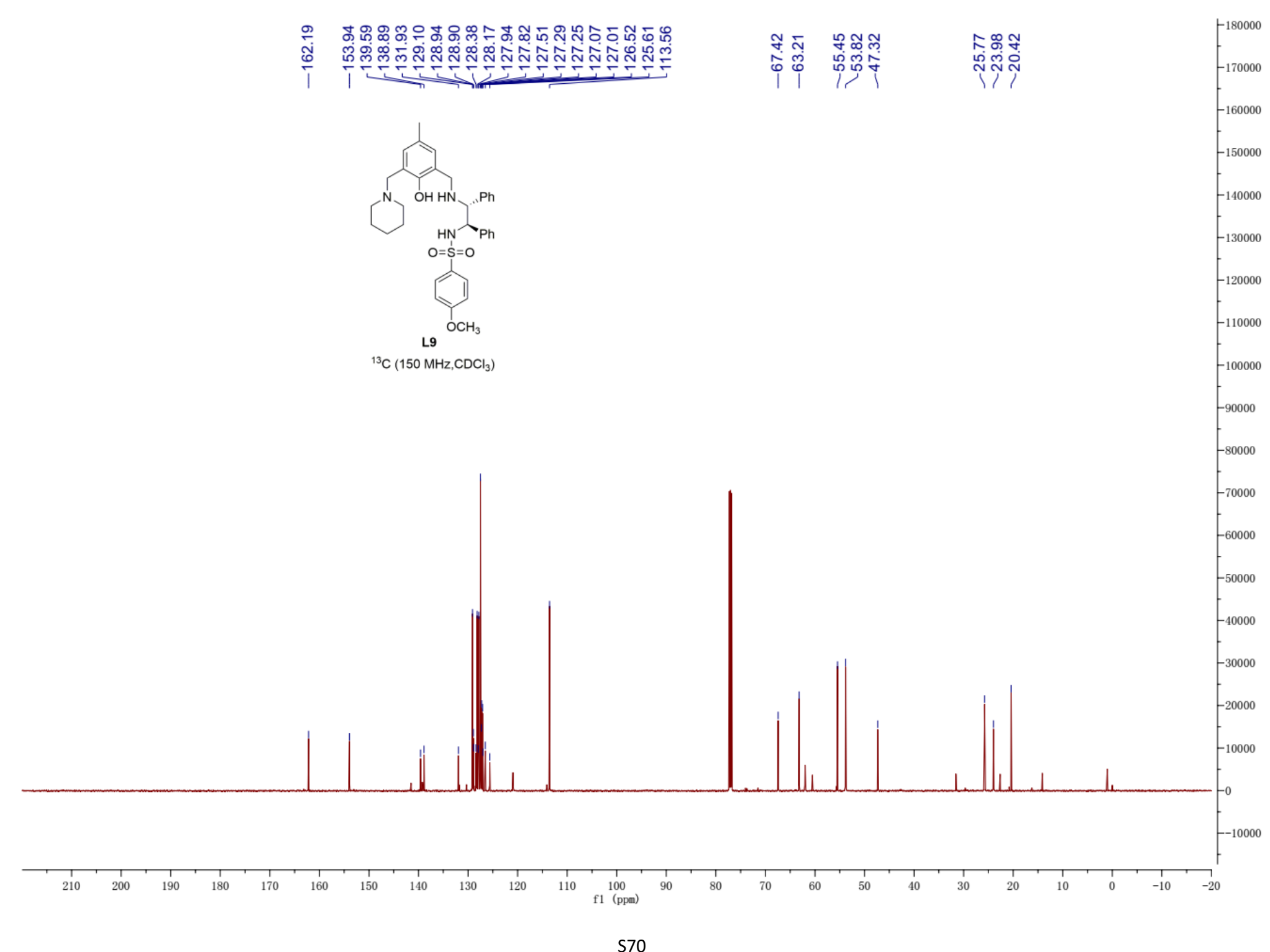




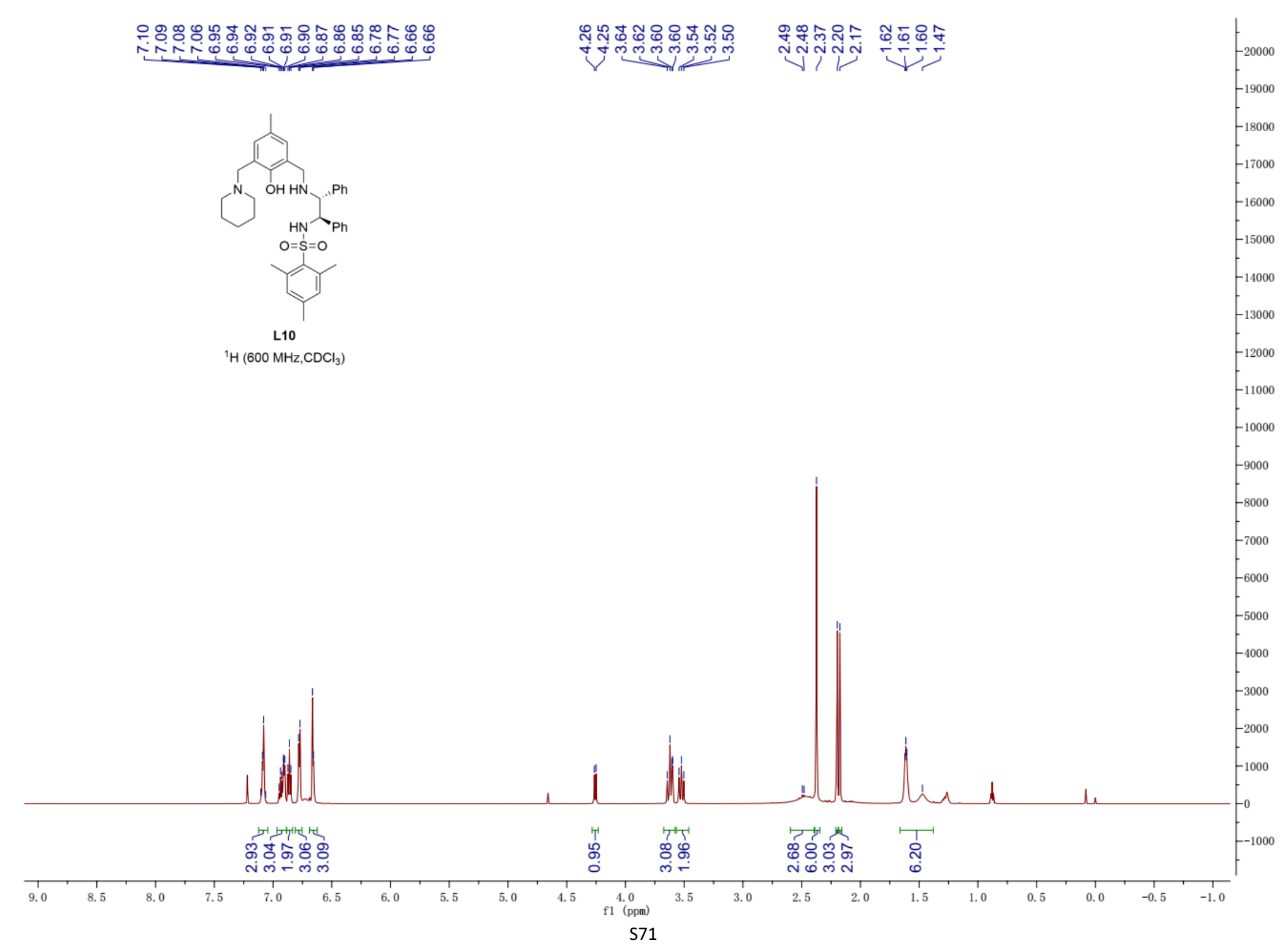




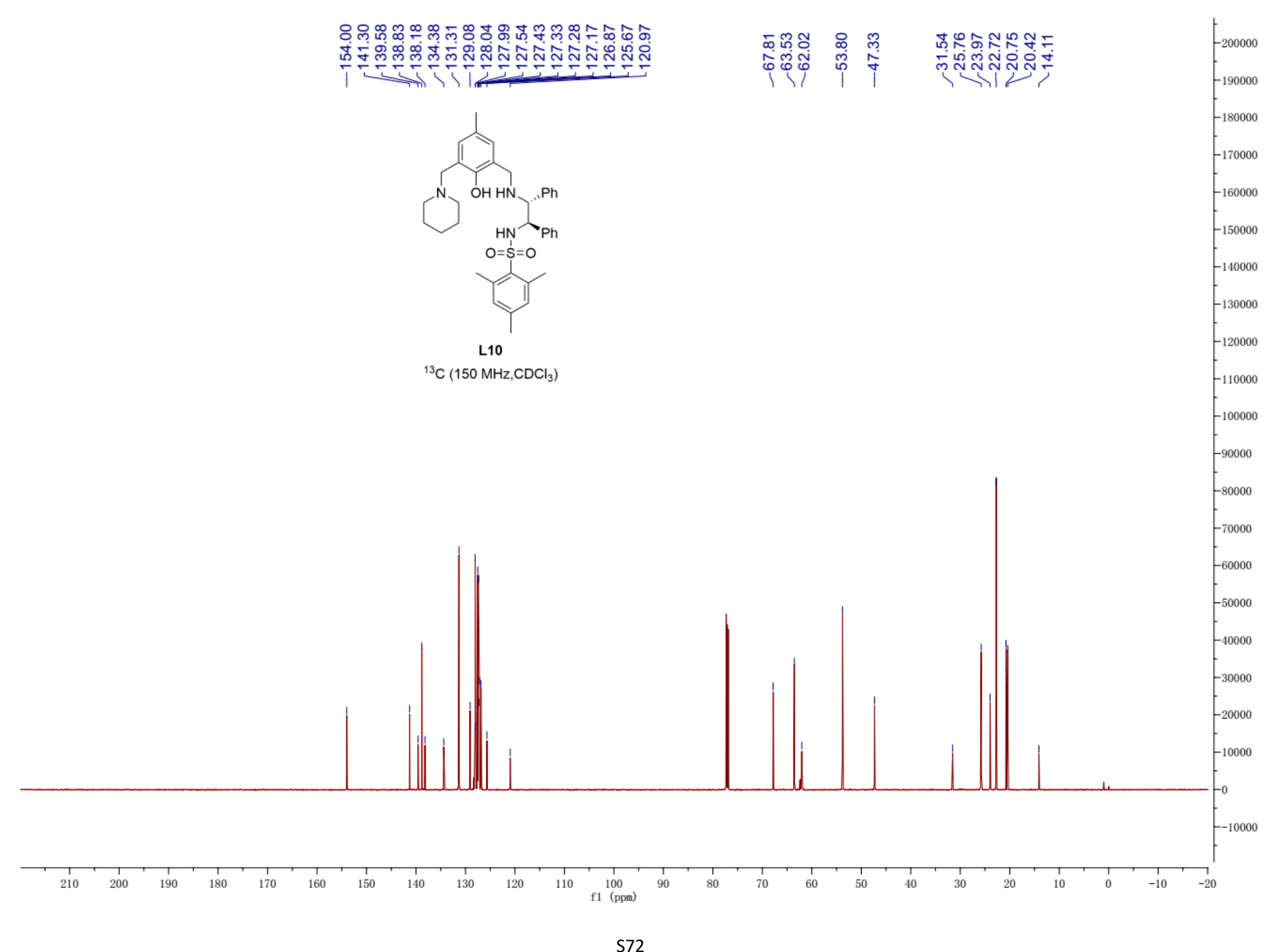




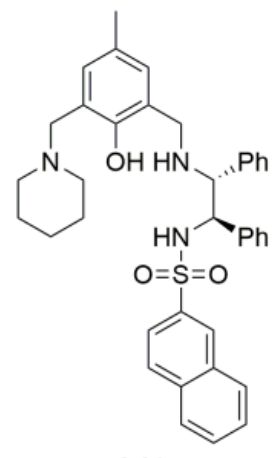

L11

${ }^{1} \mathrm{H}\left(600 \mathrm{MHz}, \mathrm{CDCl}_{3}\right)$

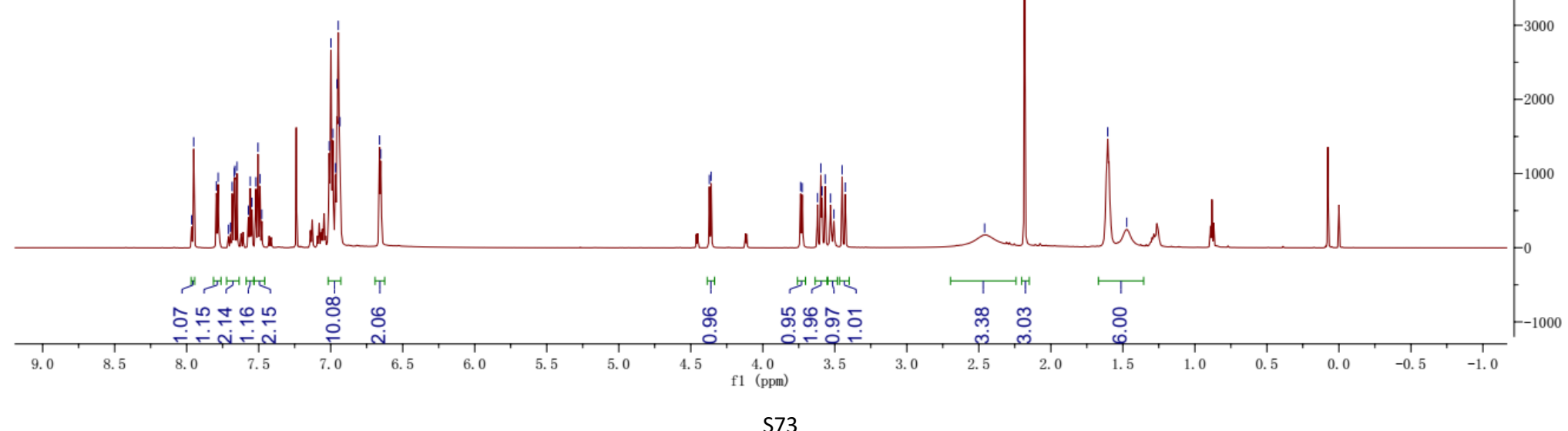




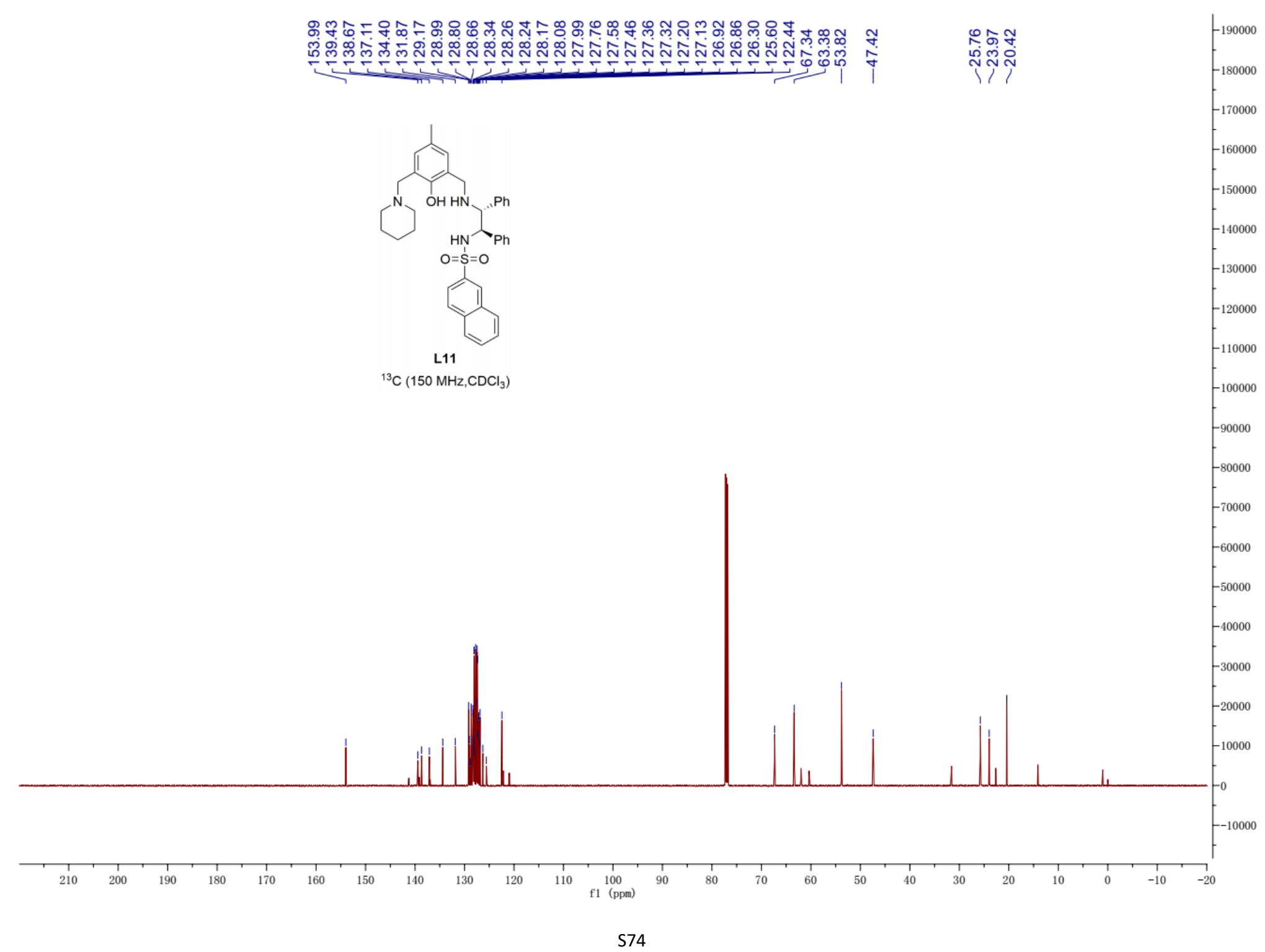




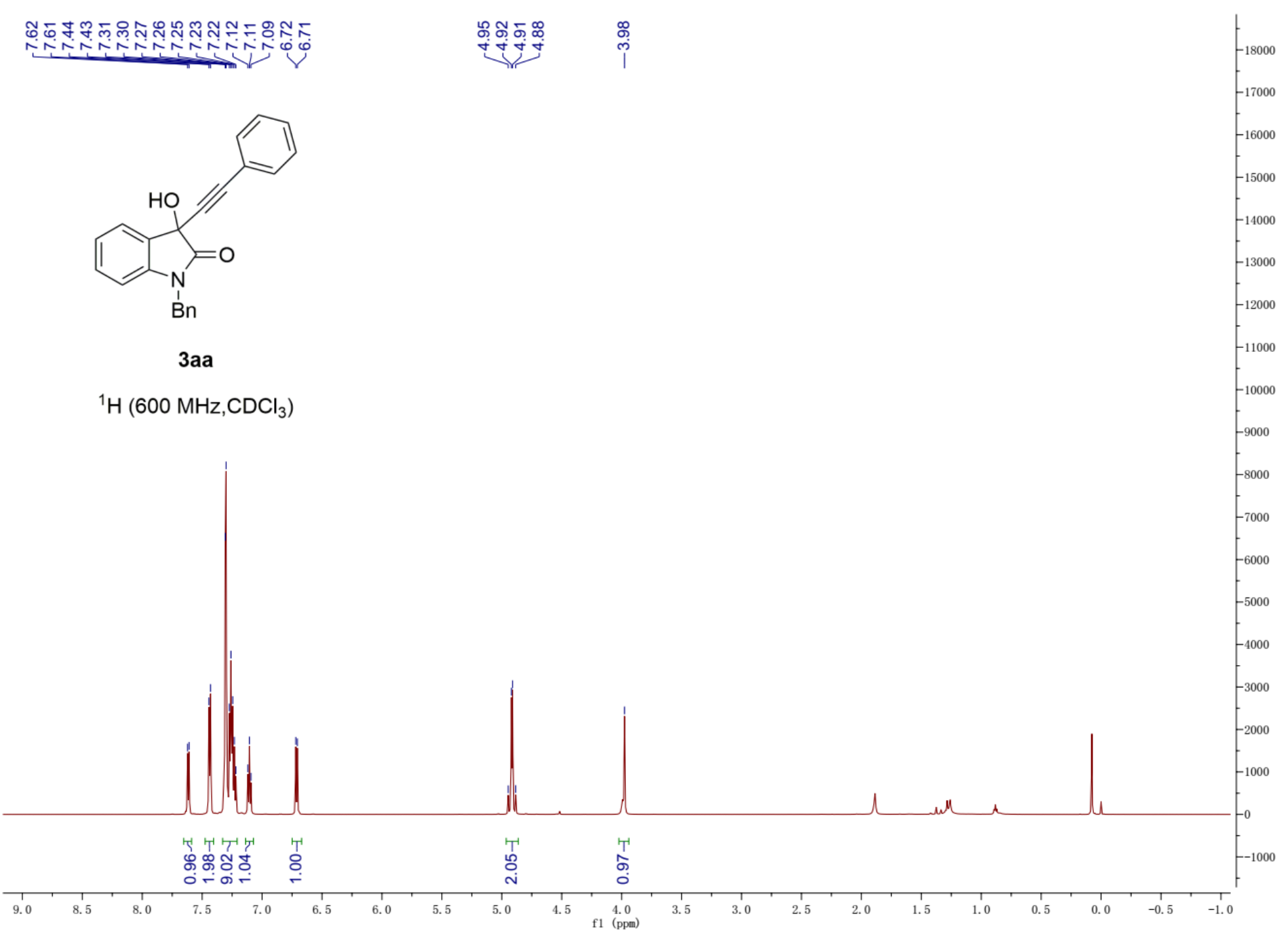




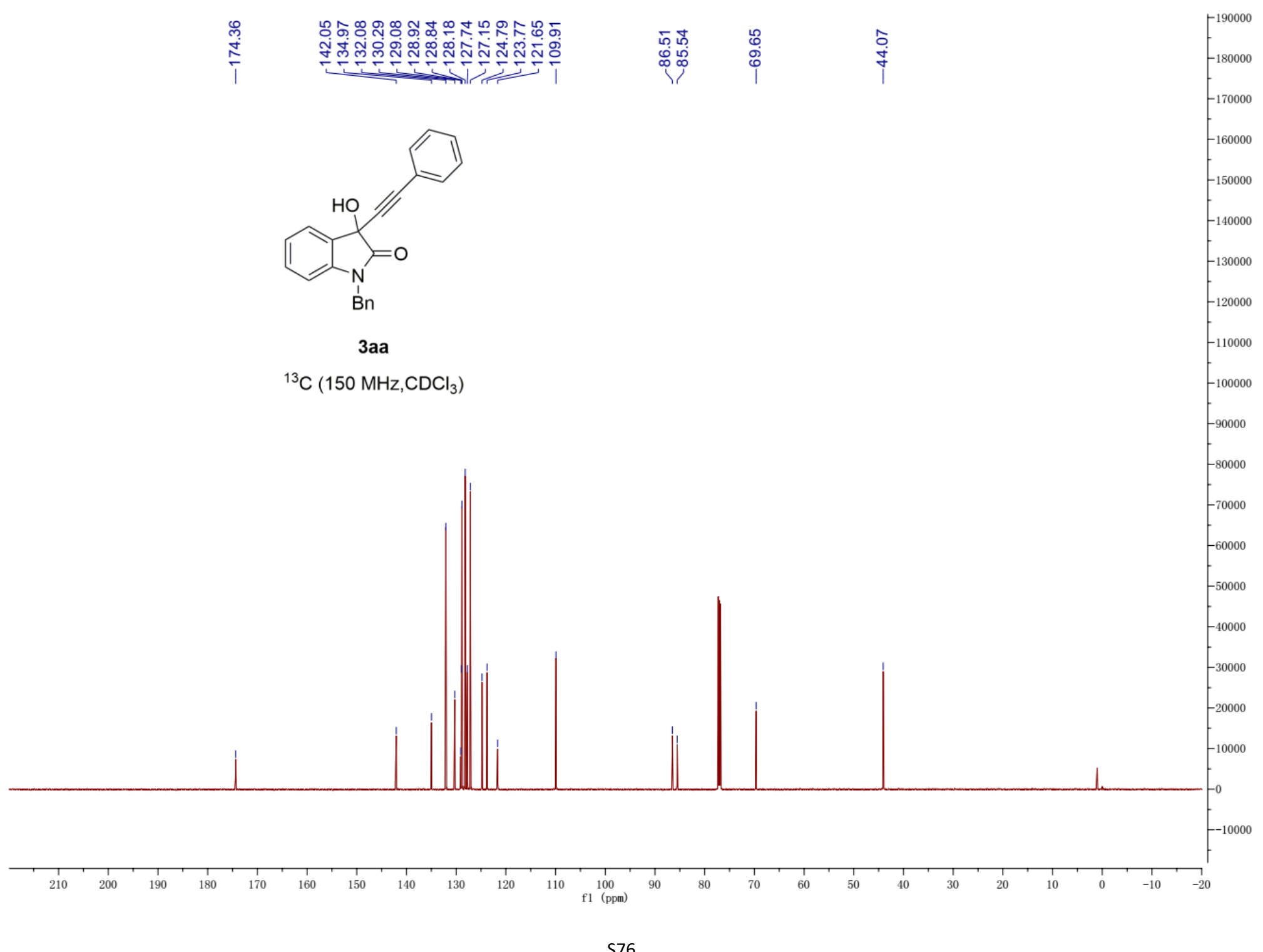




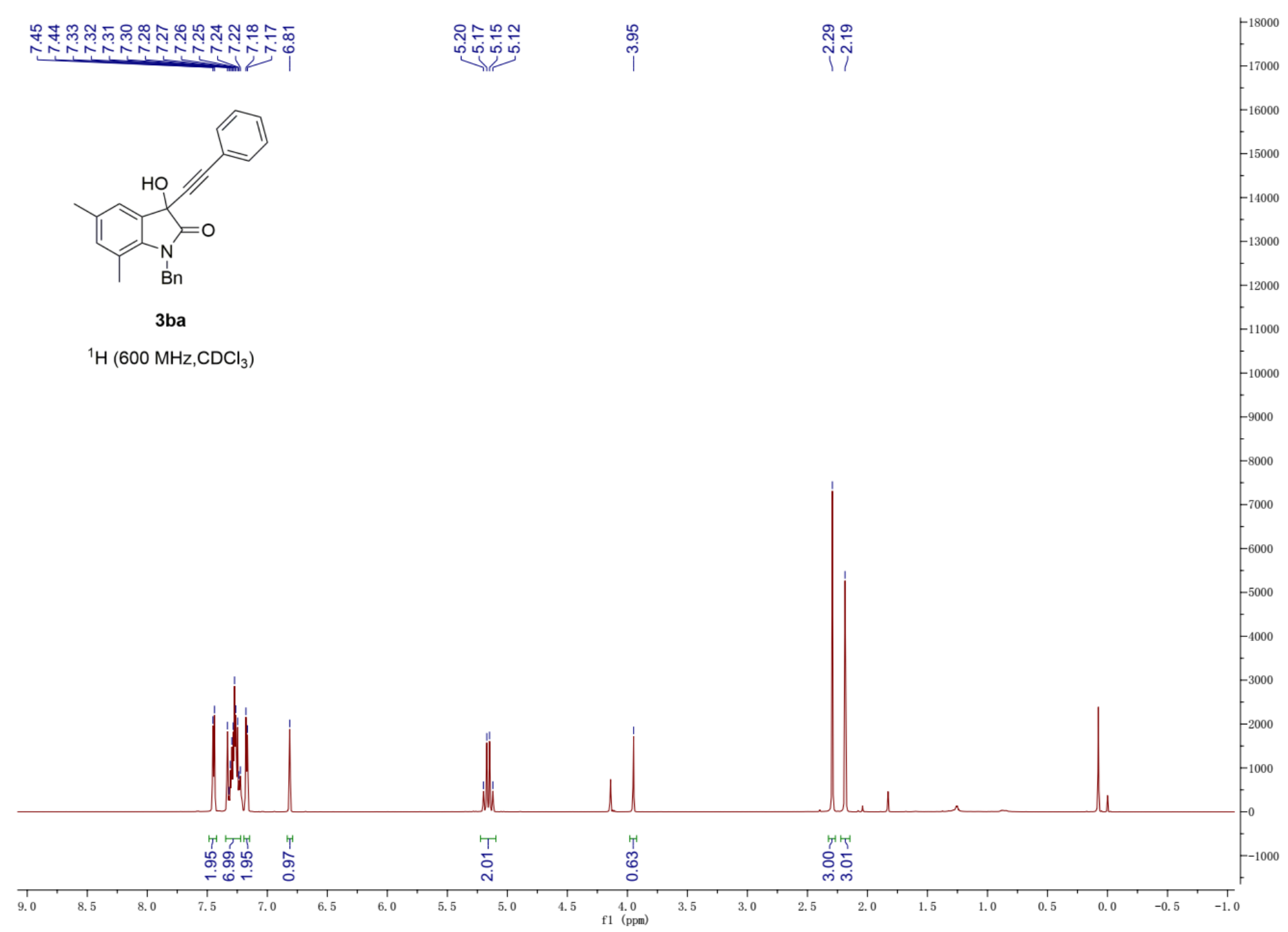




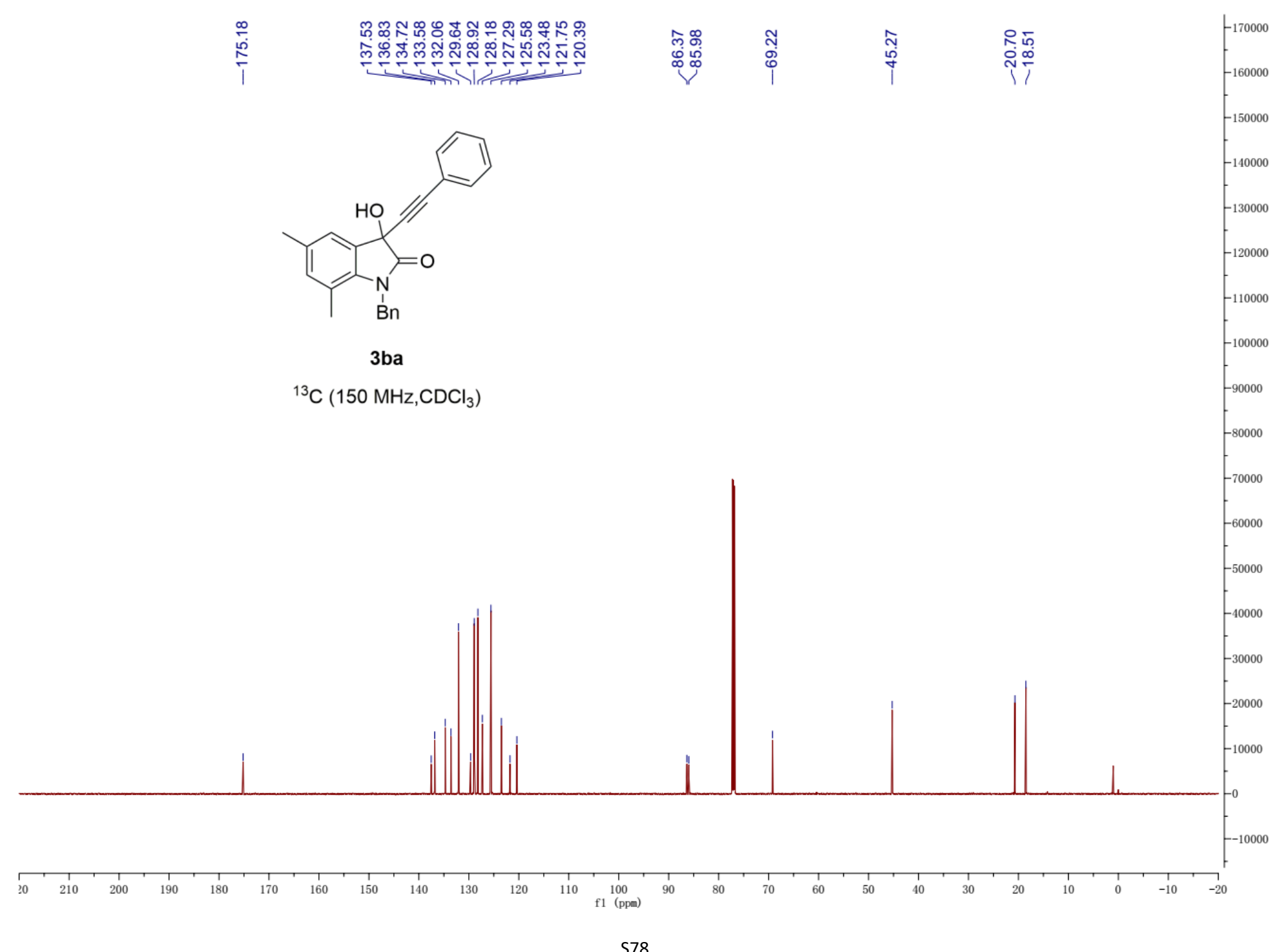




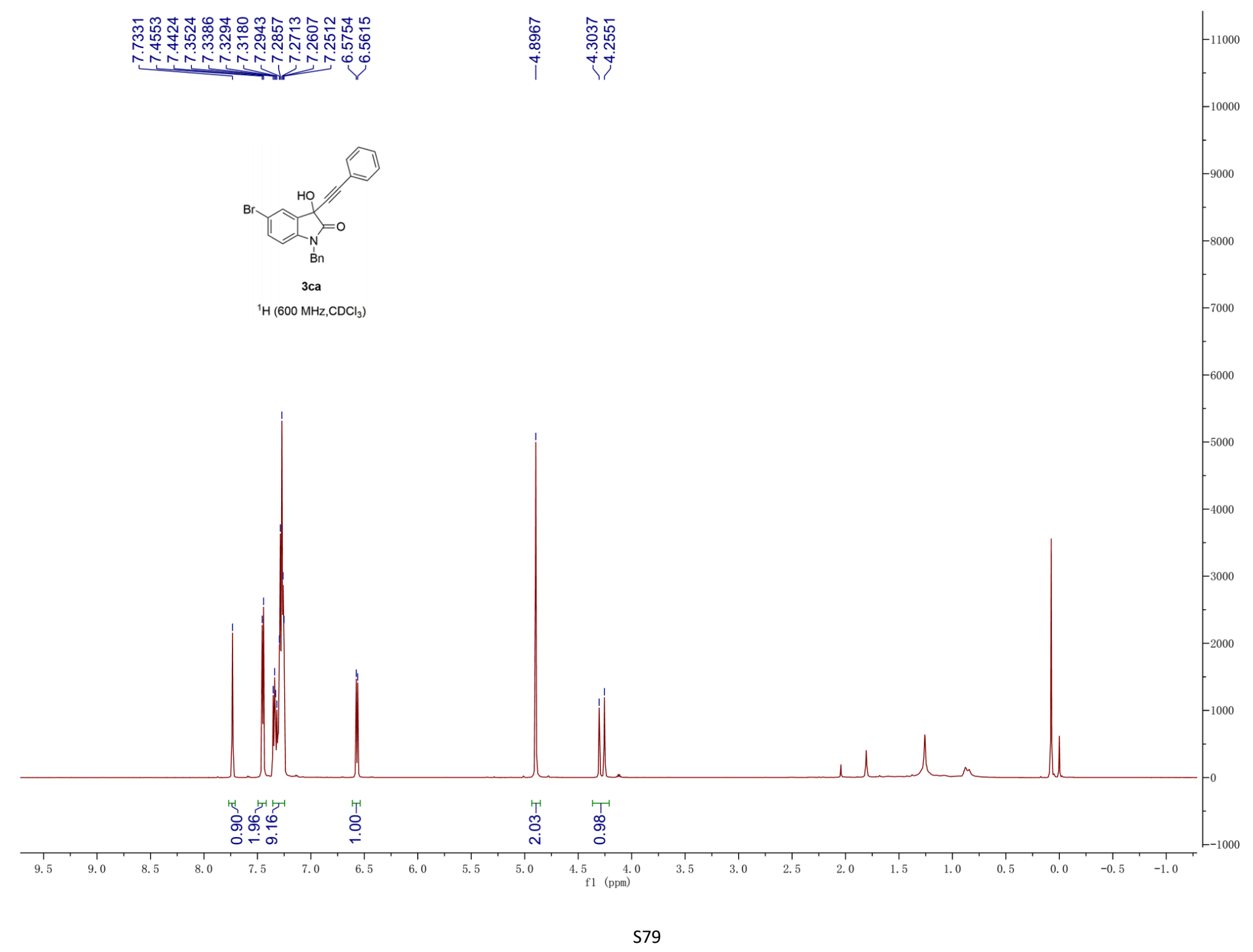


$\underbrace{0}$
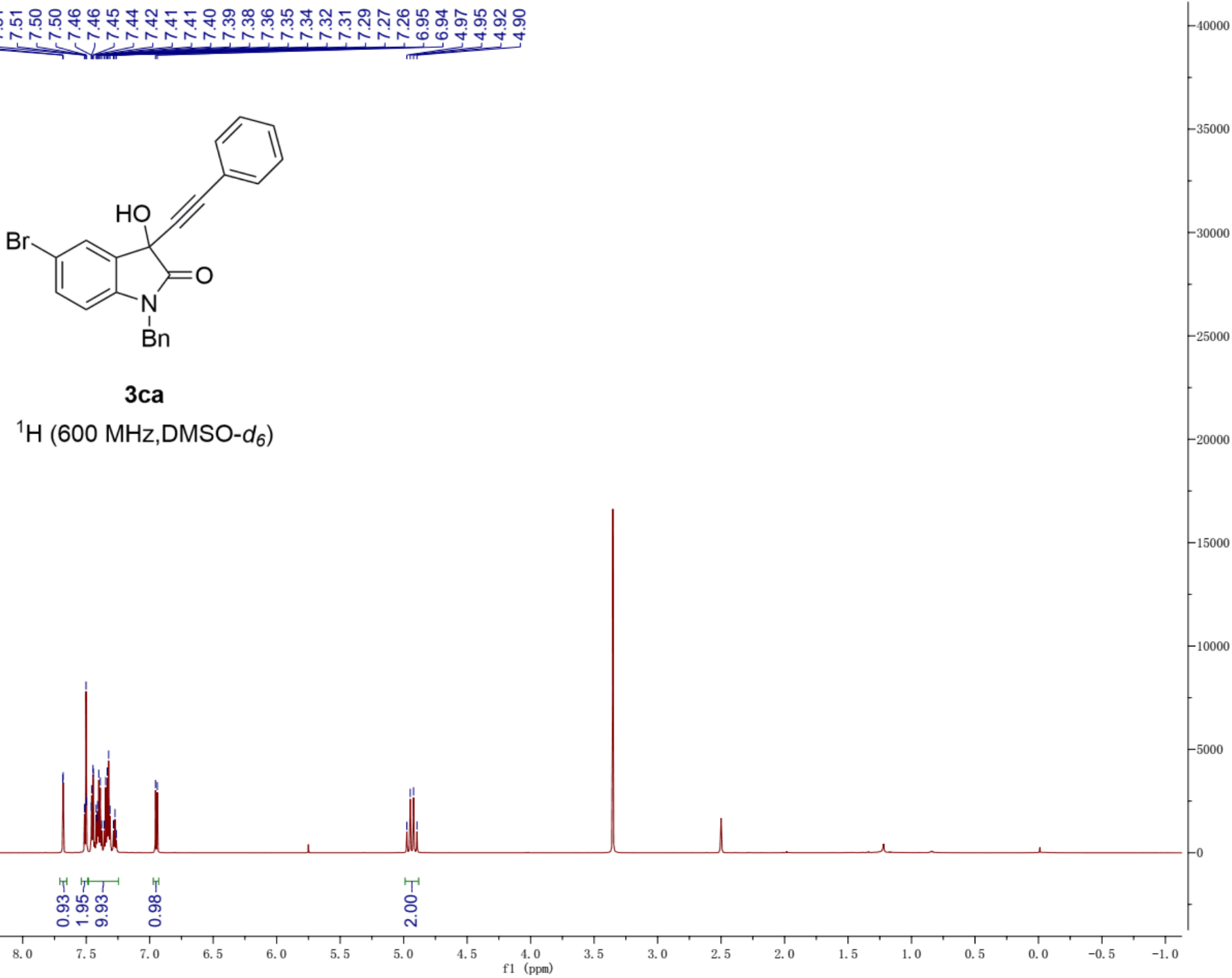


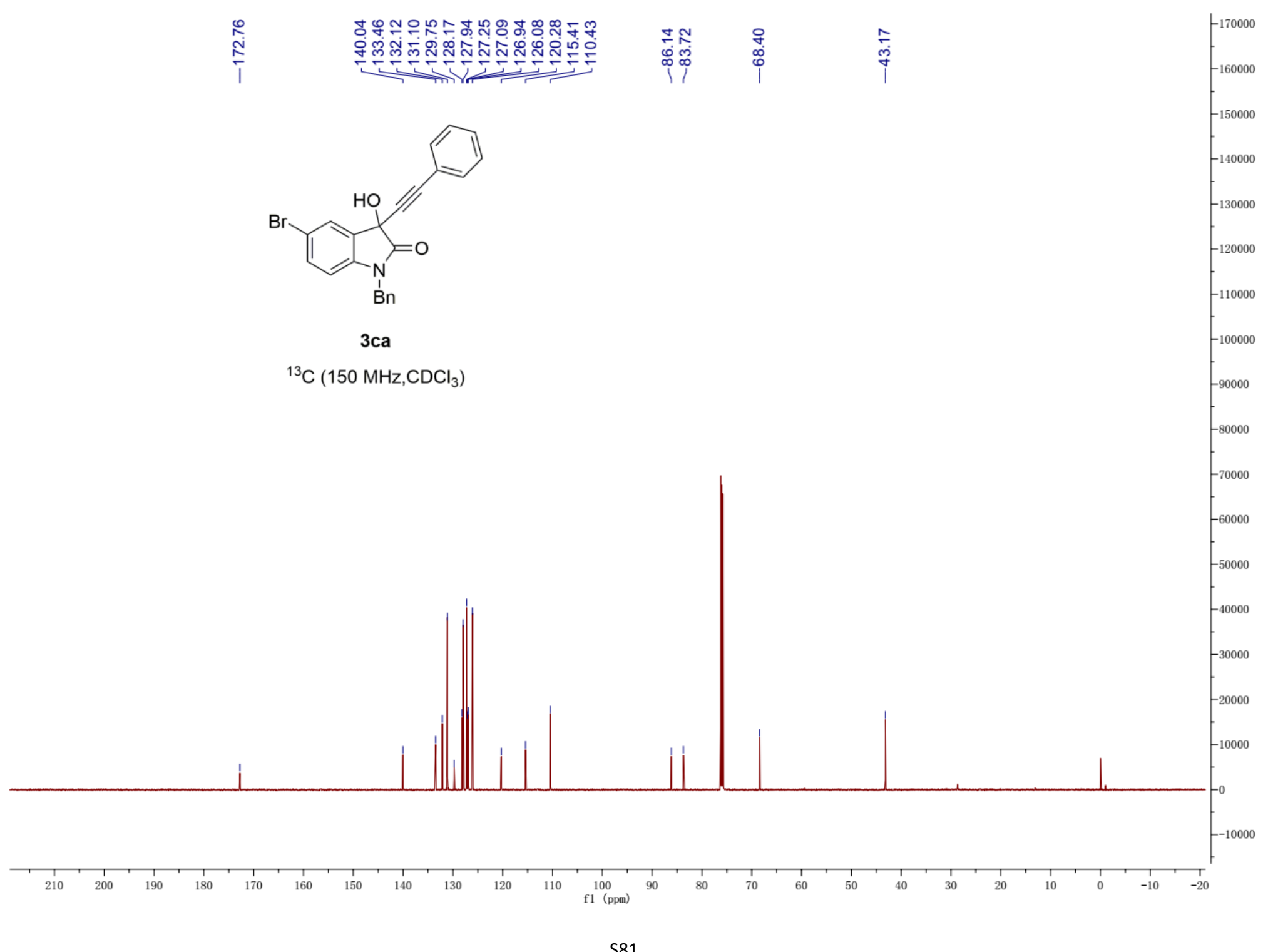




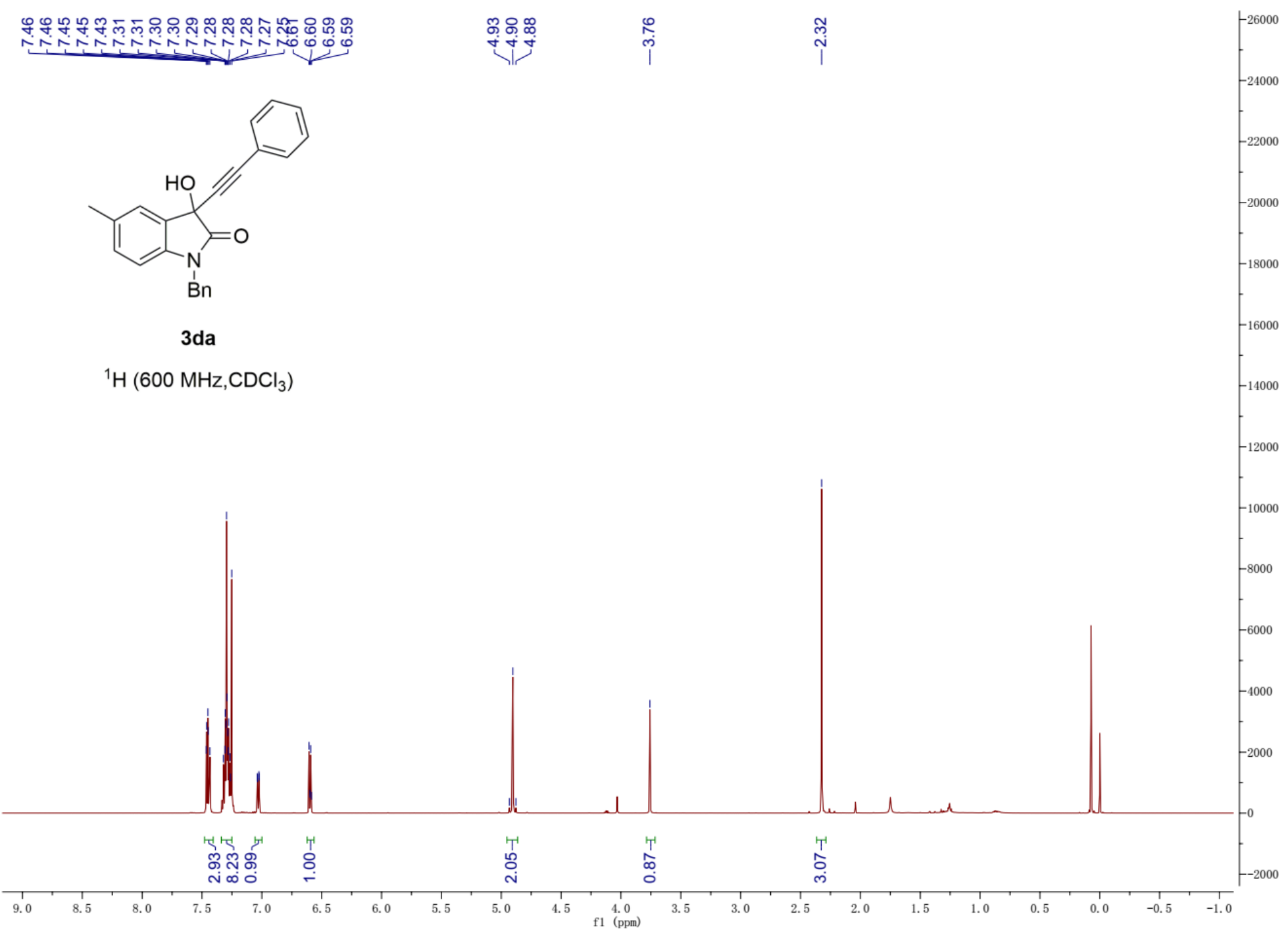



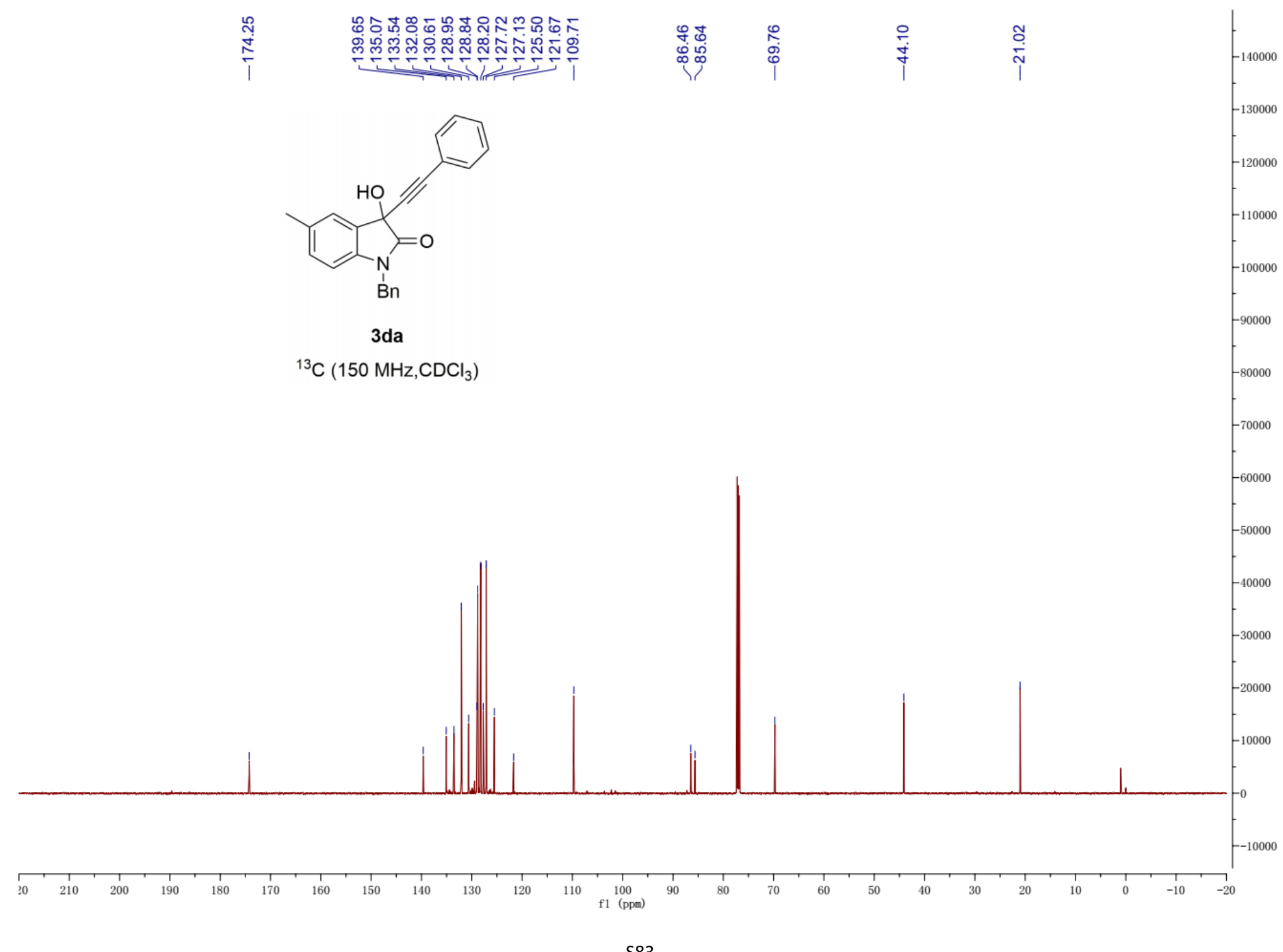


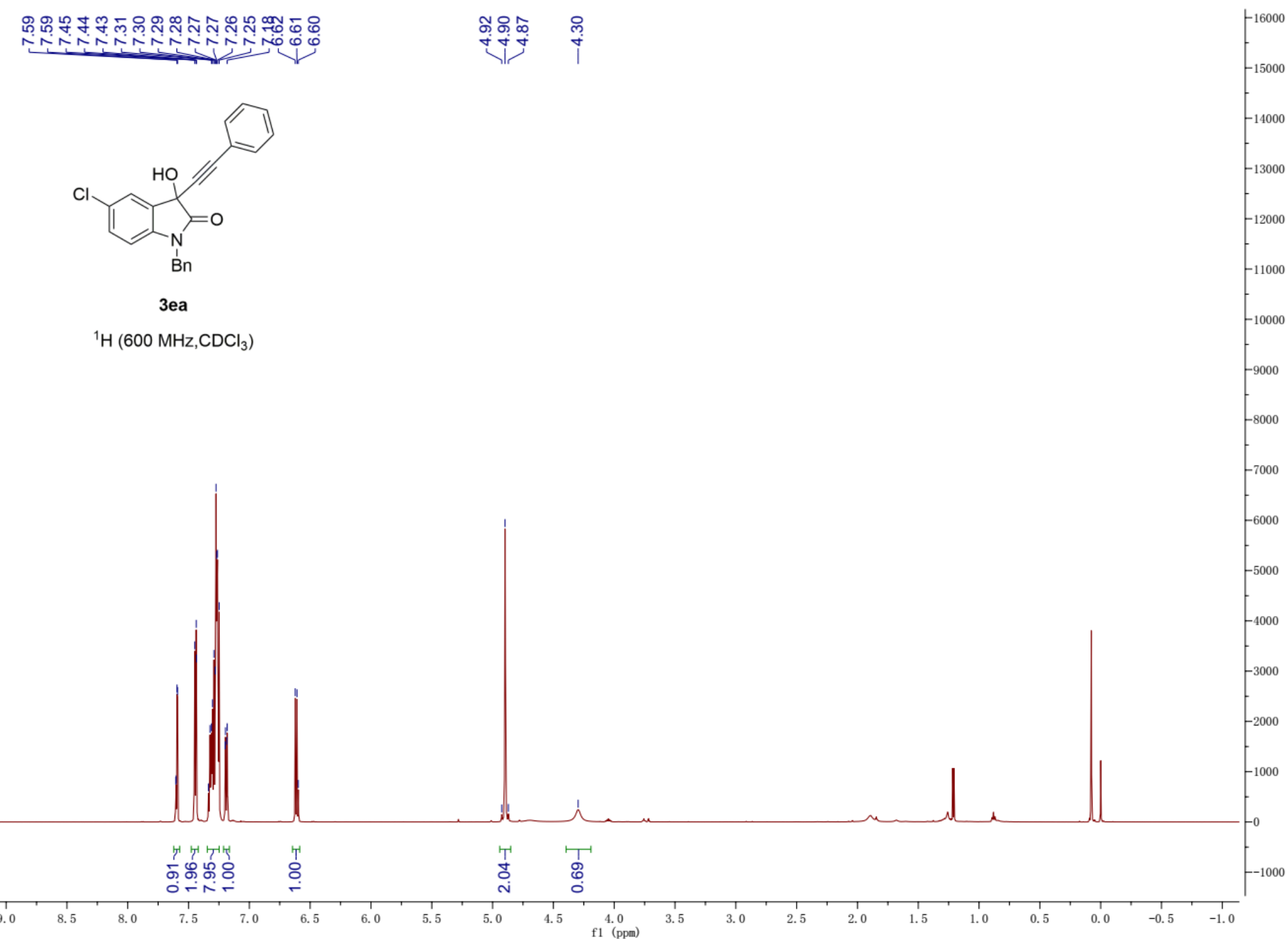




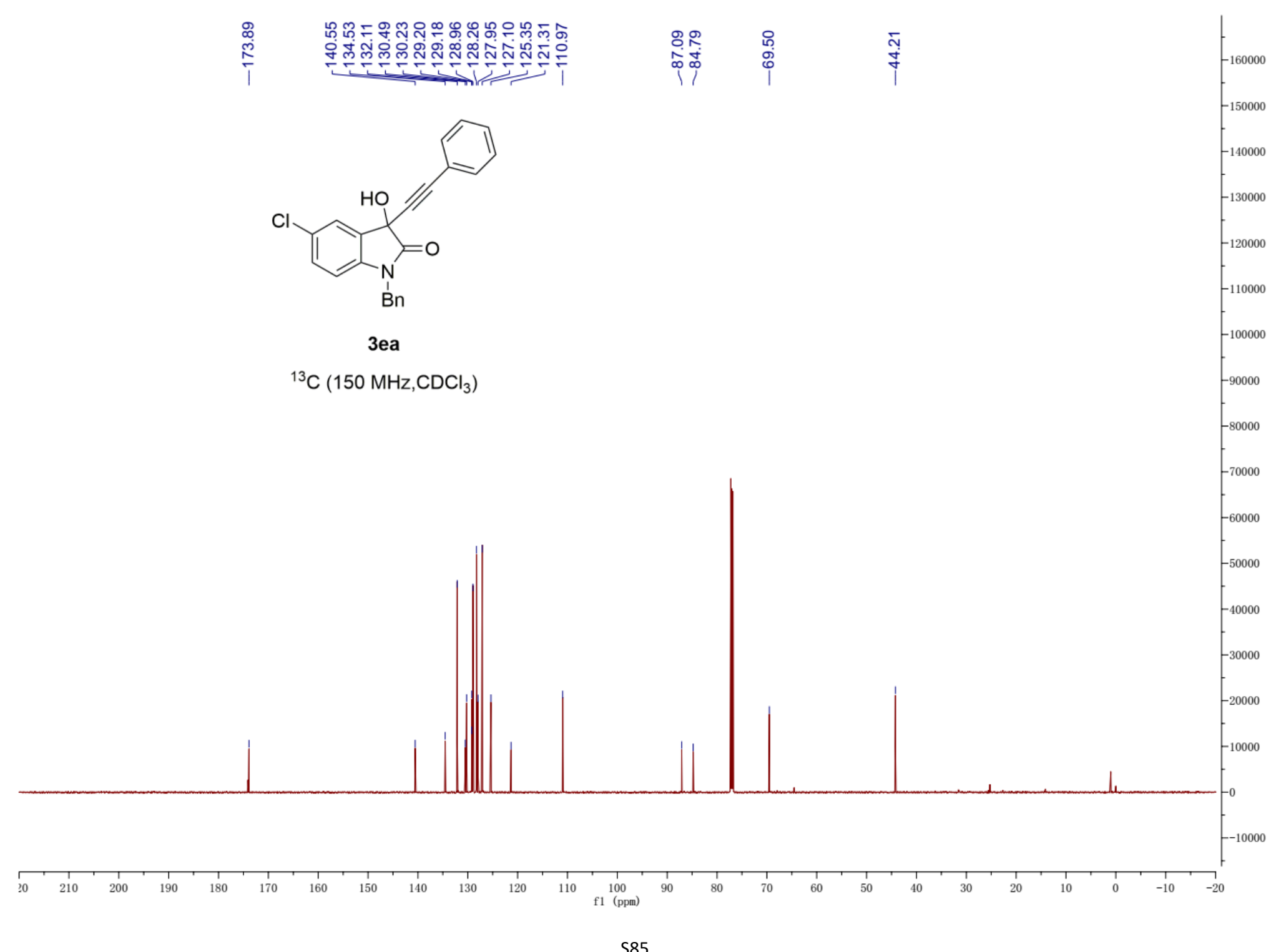

585 


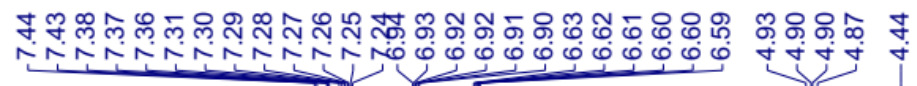

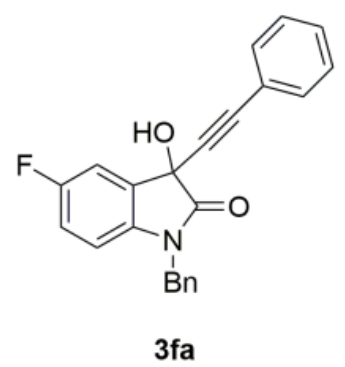

${ }^{1} \mathrm{H}\left(600 \mathrm{MHz}, \mathrm{CDCl}_{3}\right)$

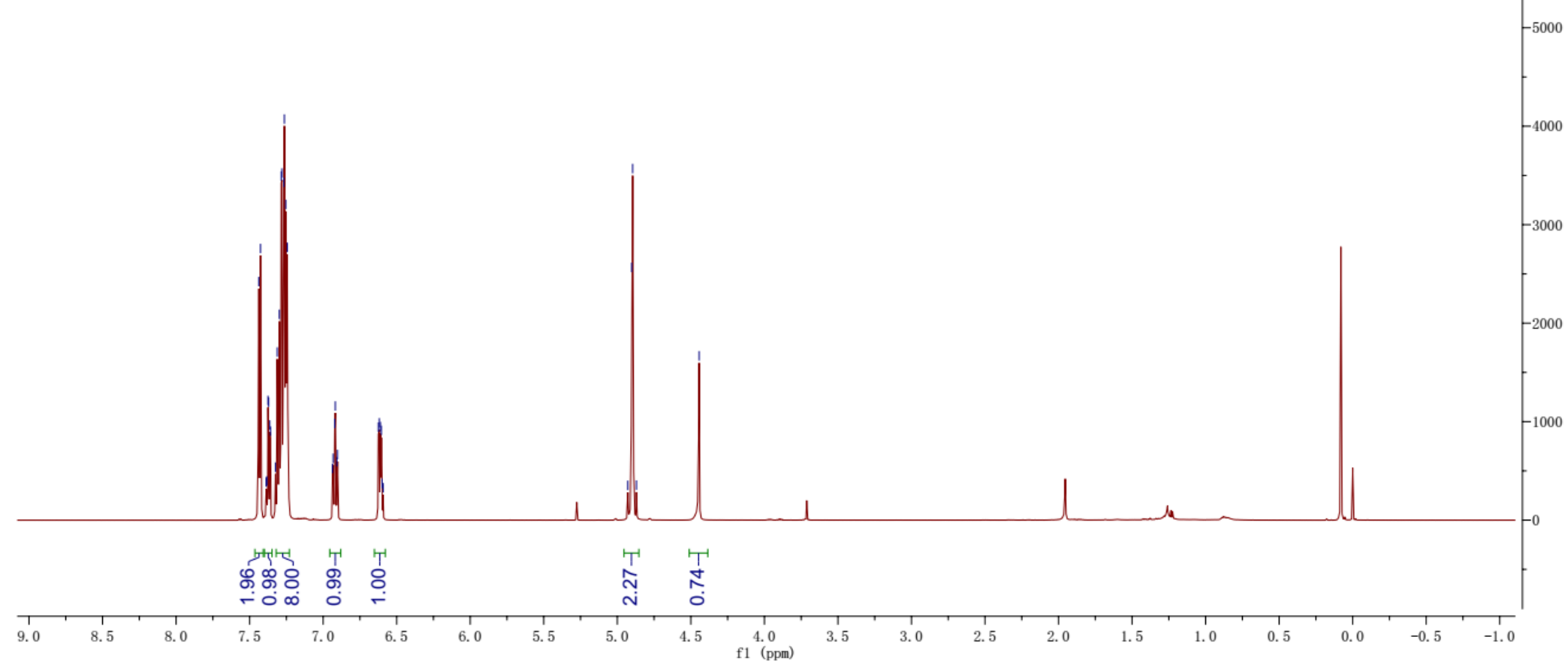




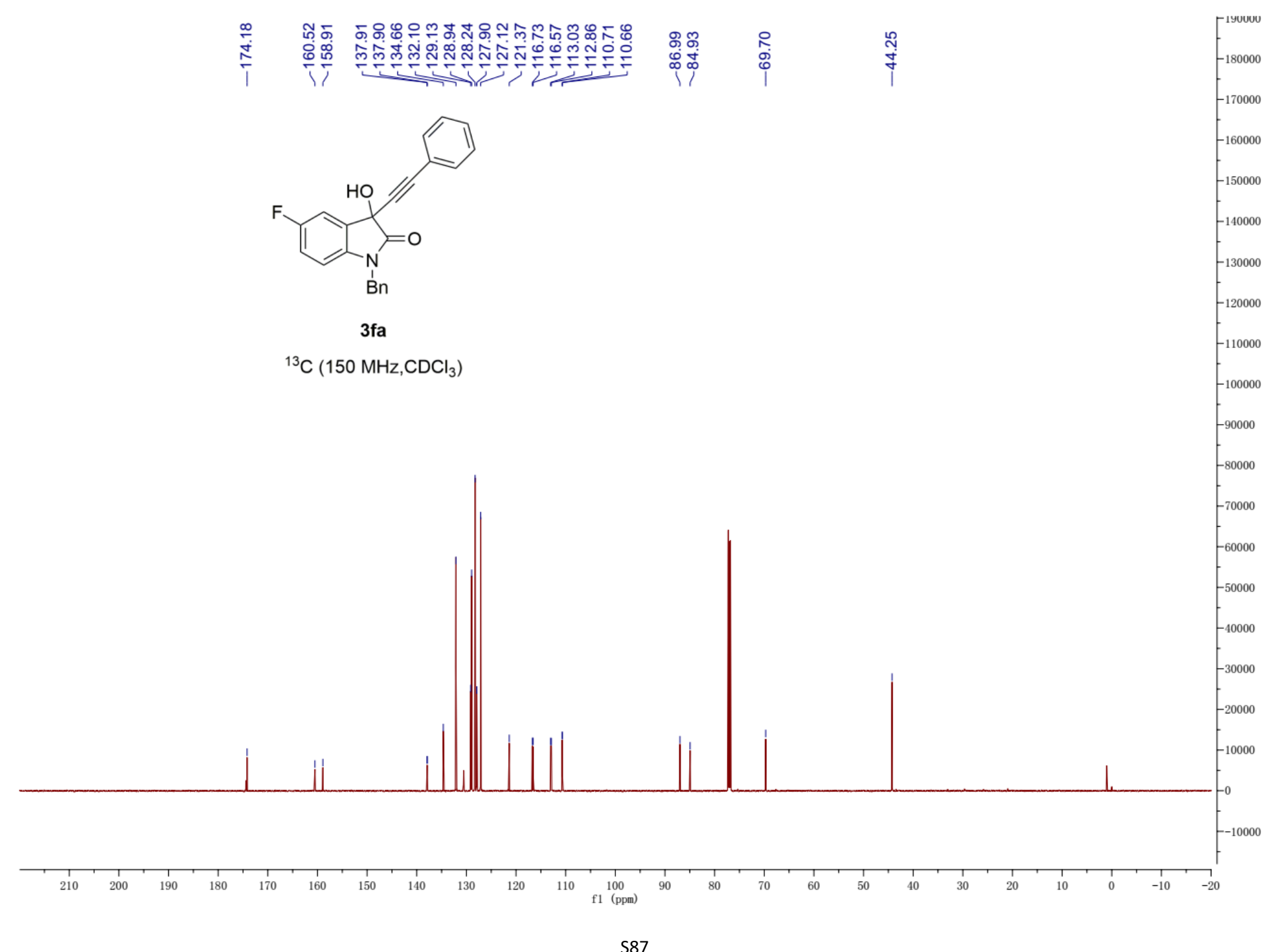

S87 


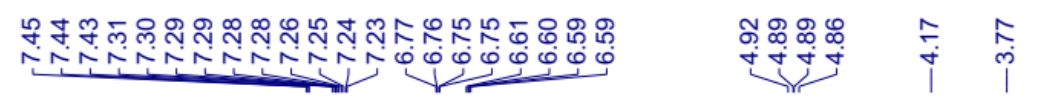

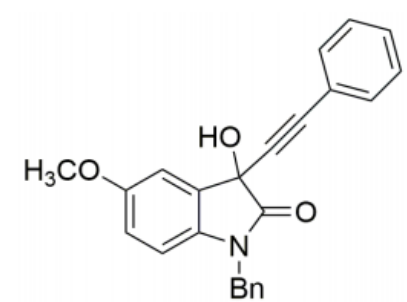

3ga

${ }^{1} \mathrm{H}\left(600 \mathrm{MHz}, \mathrm{CDCl}_{3}\right)$

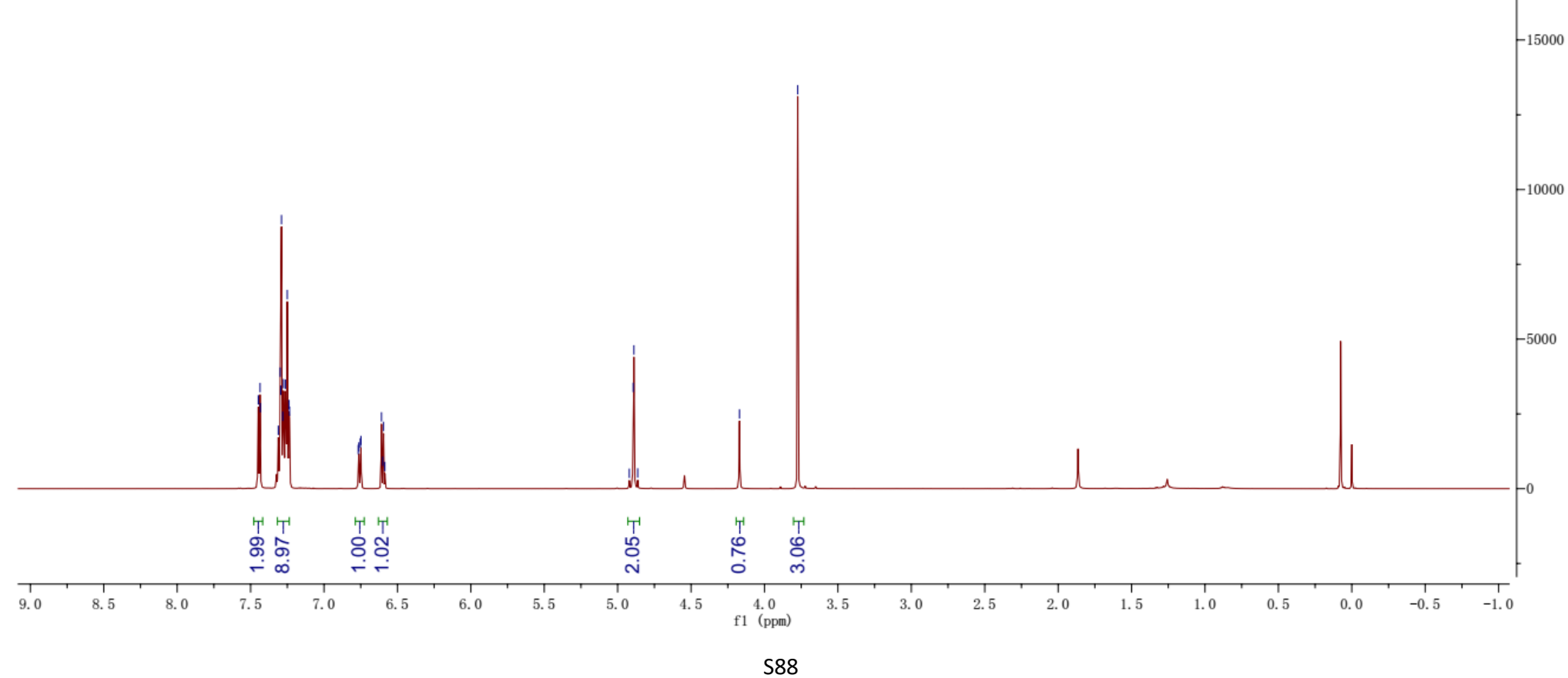




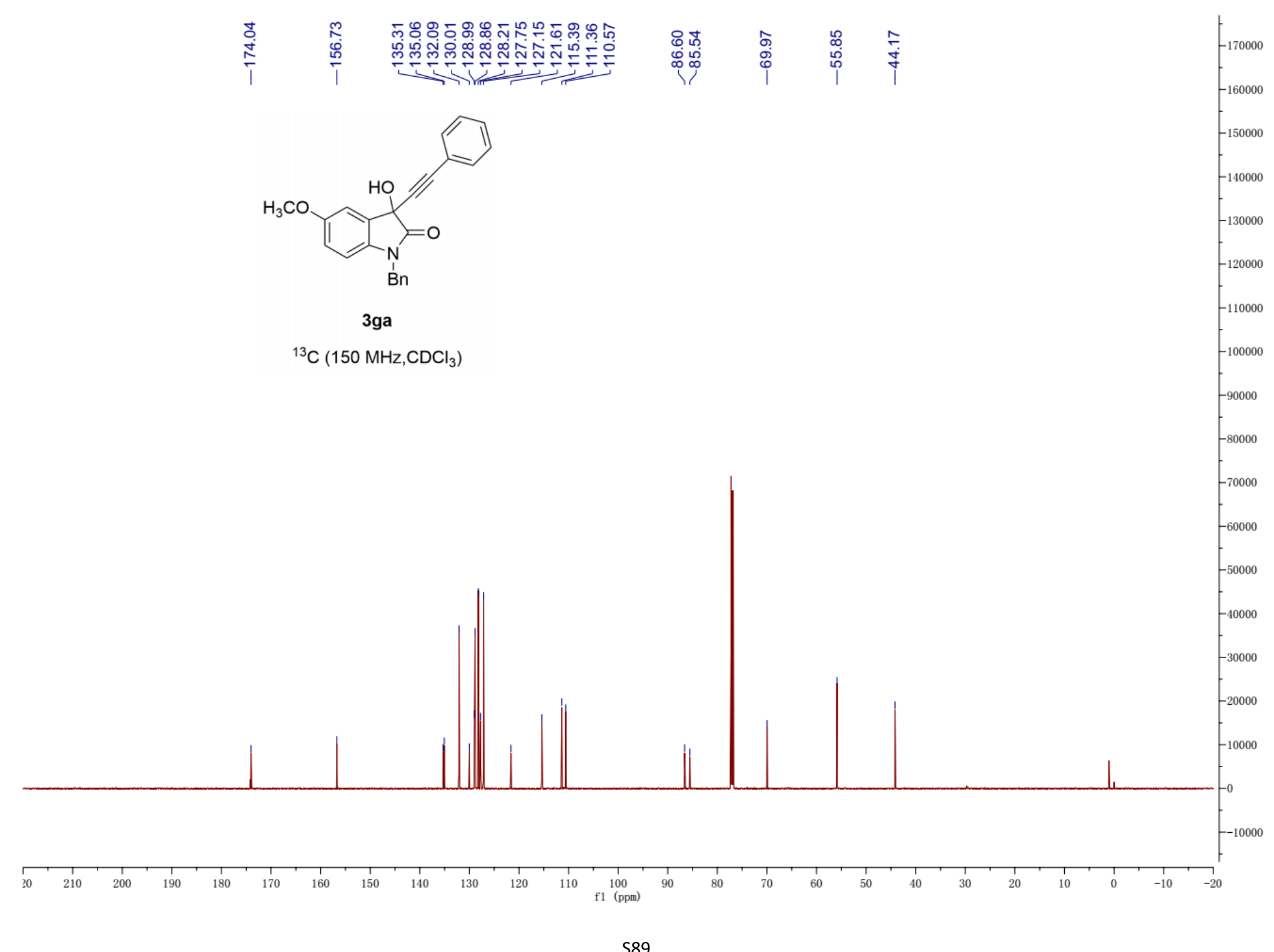




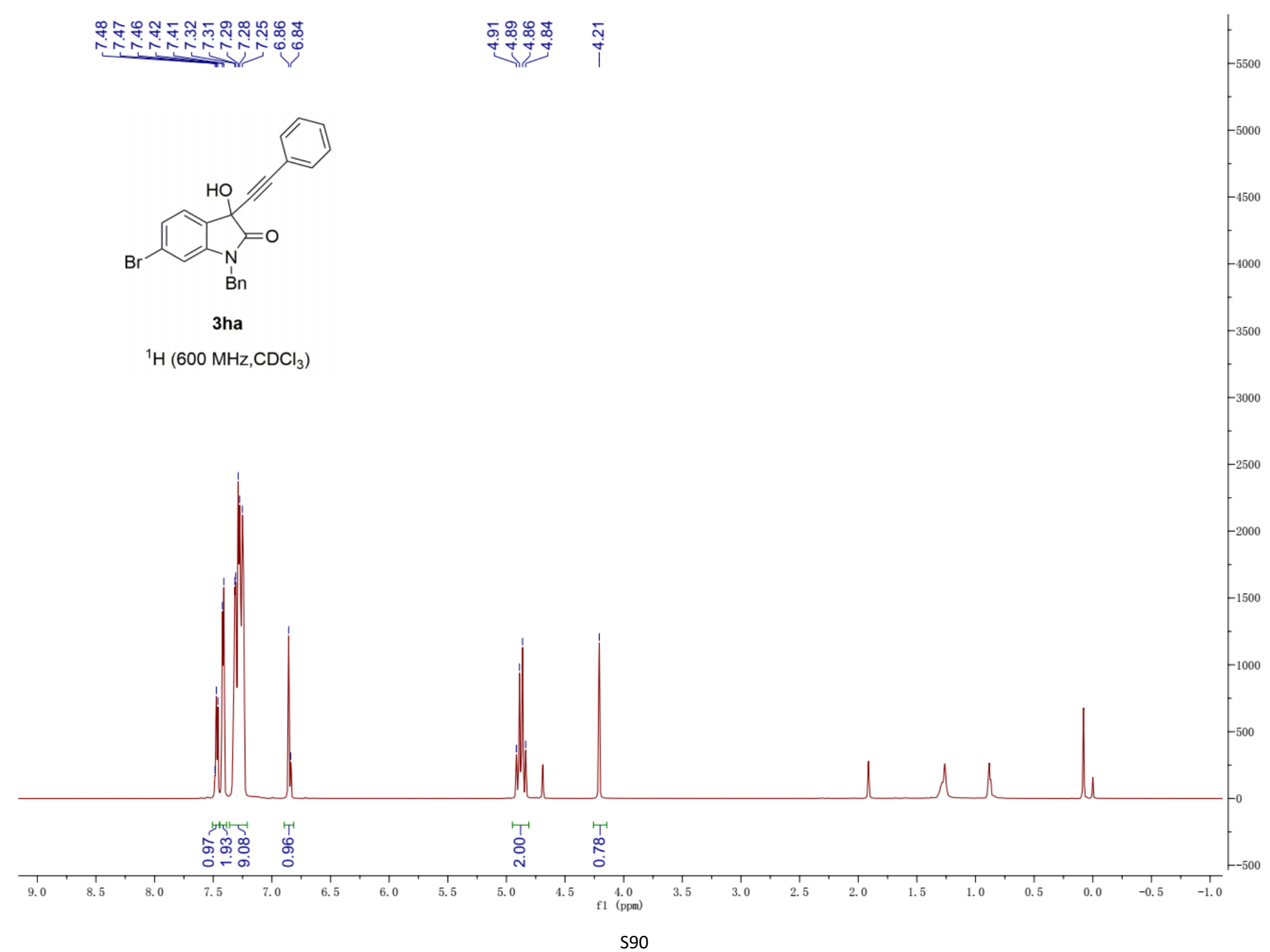




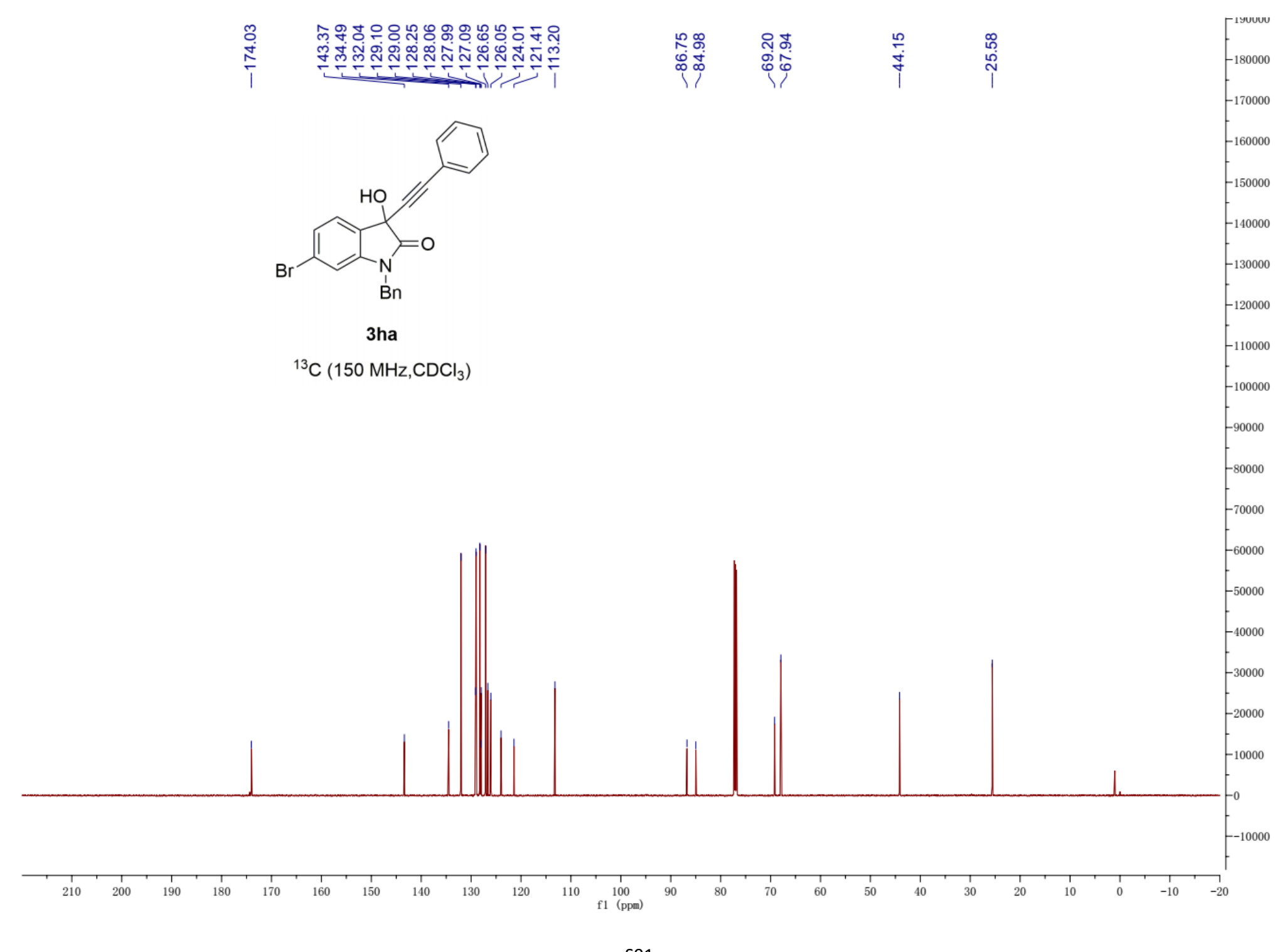

S91 


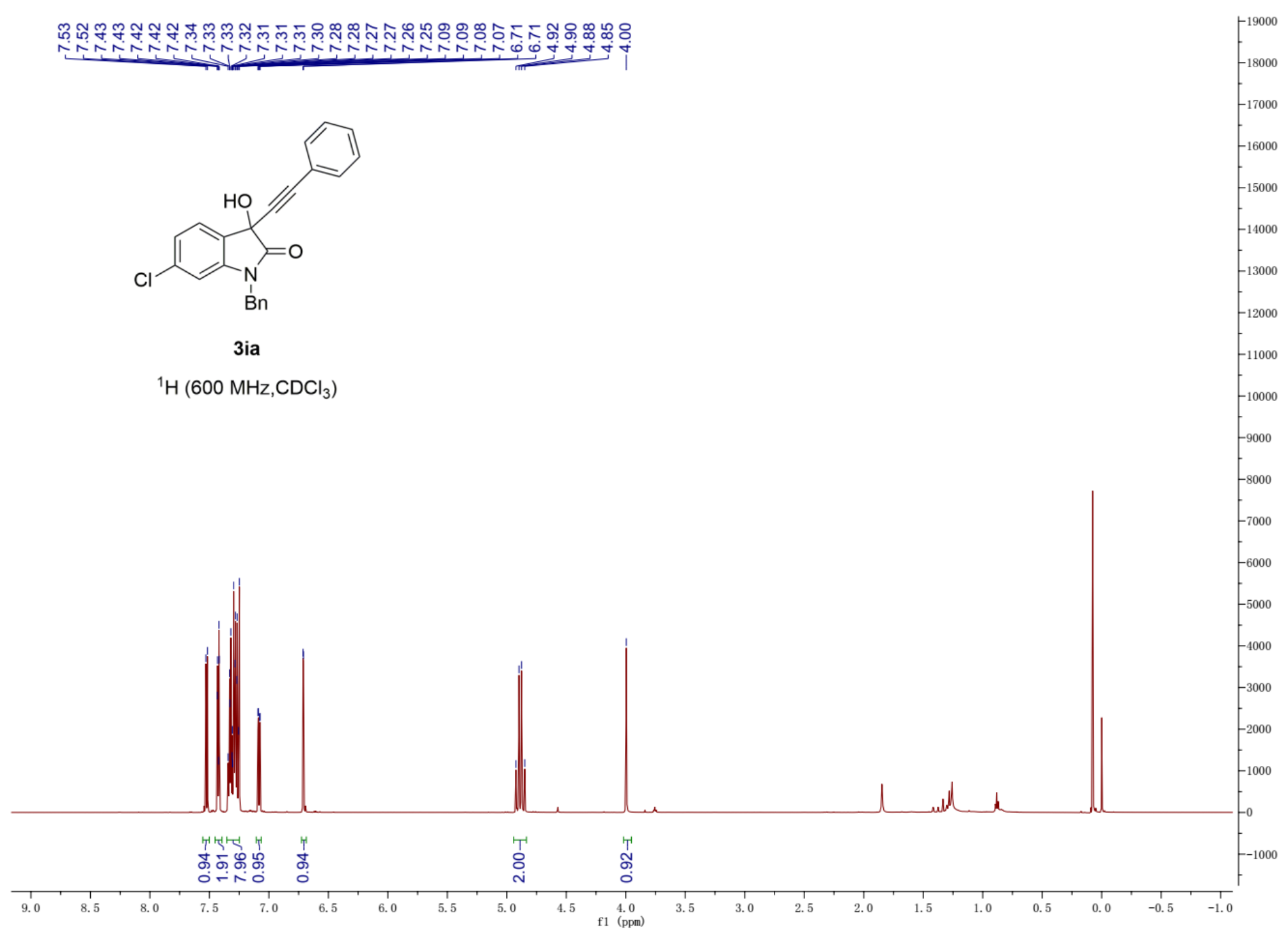




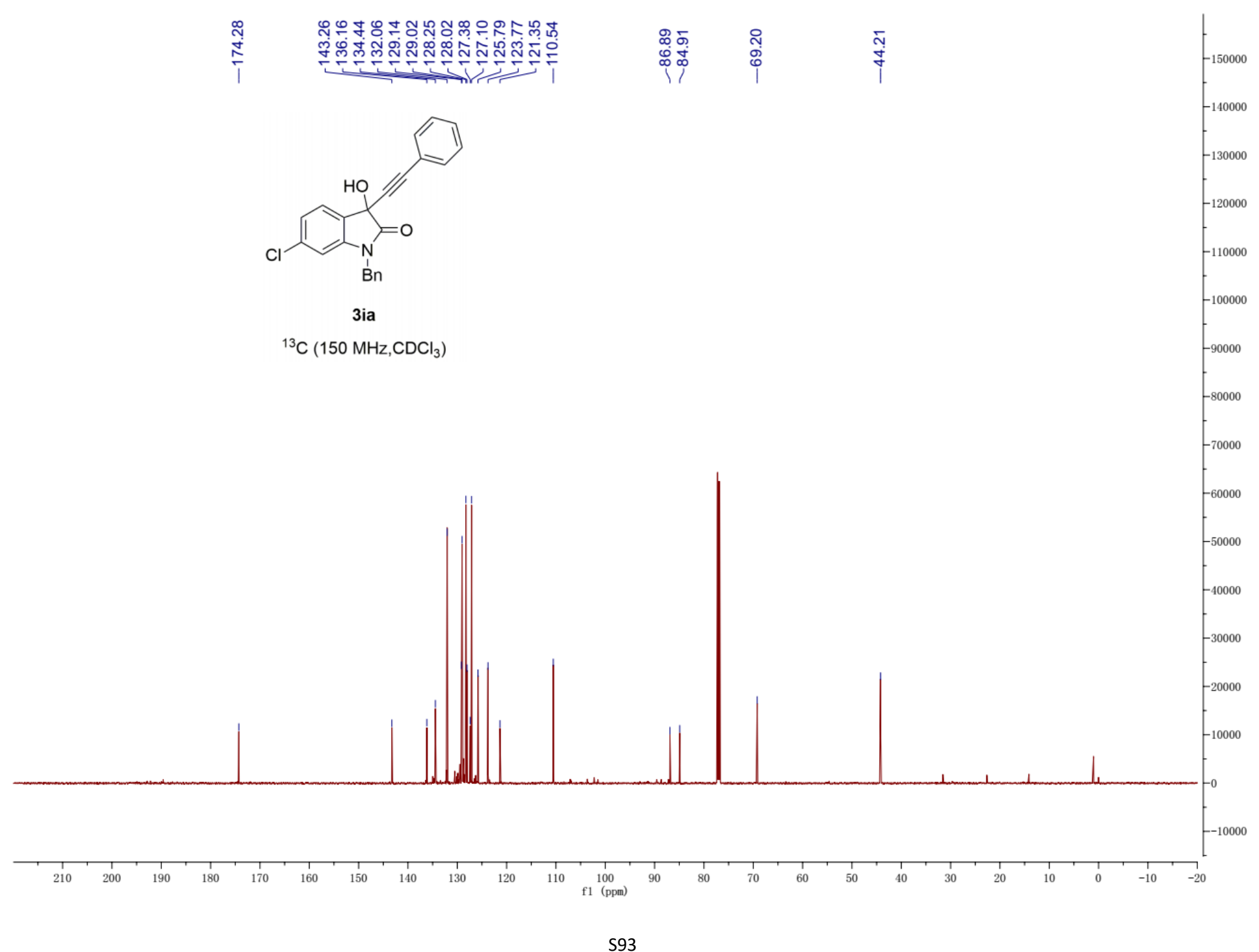




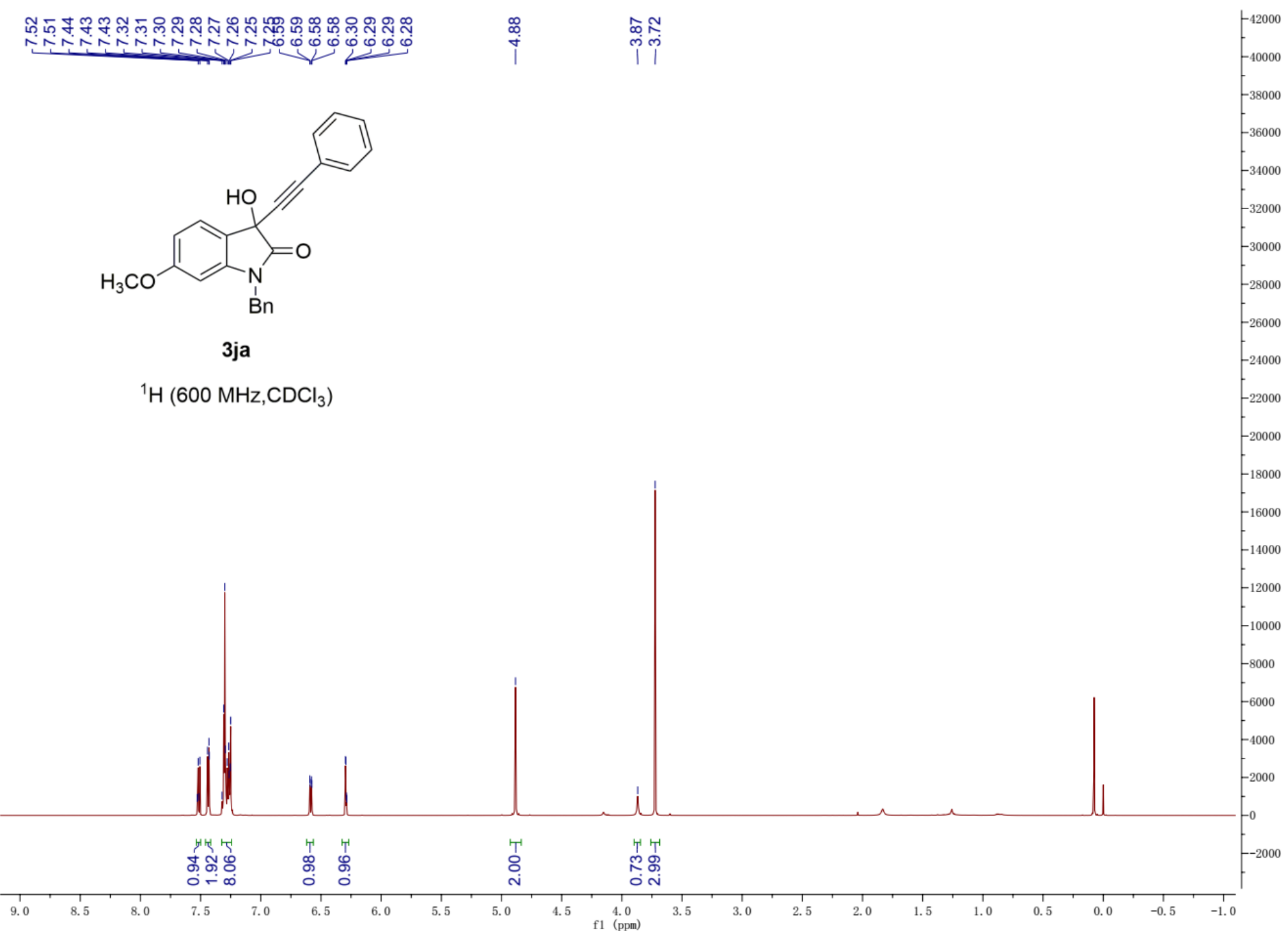

594 


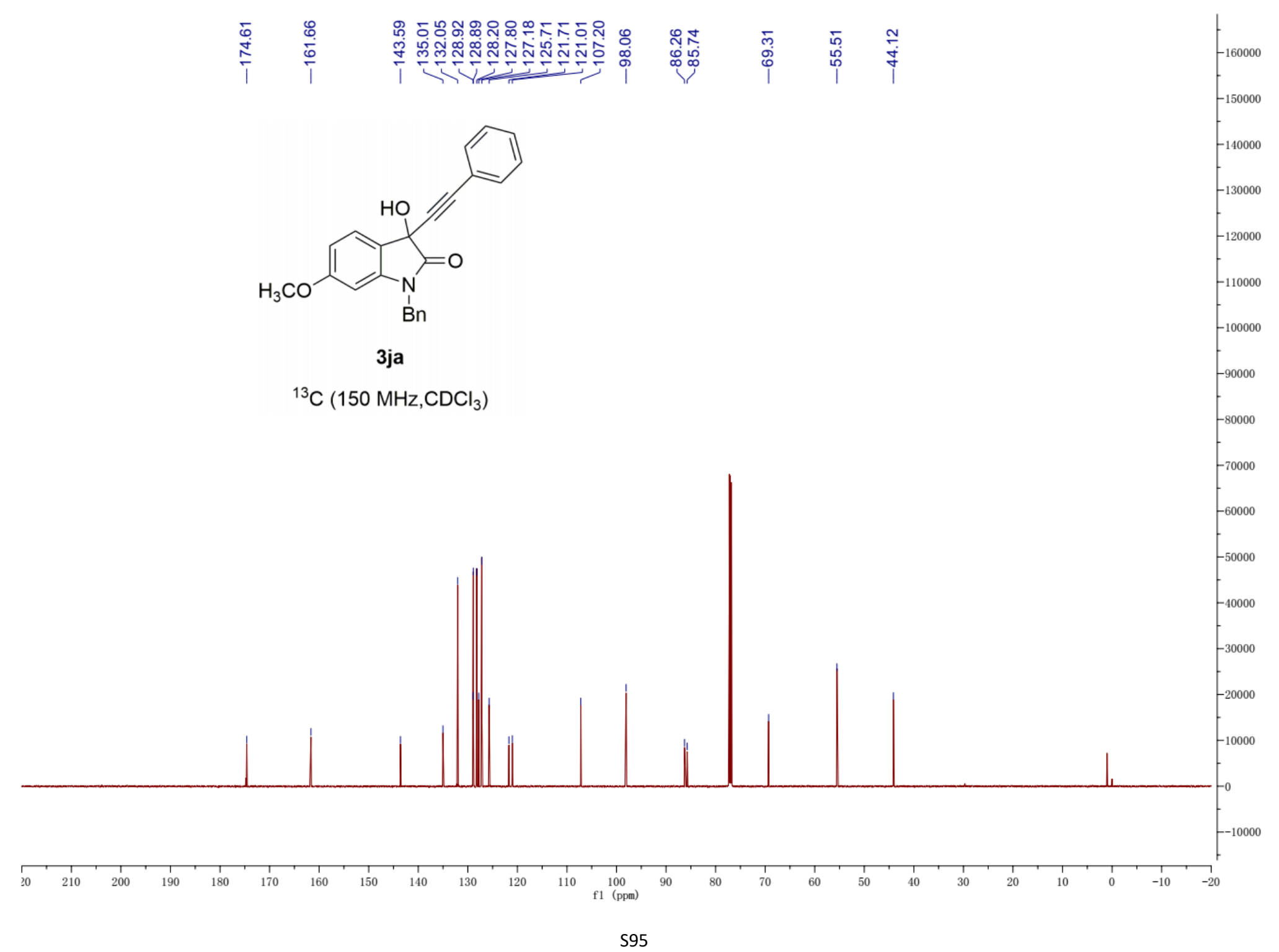




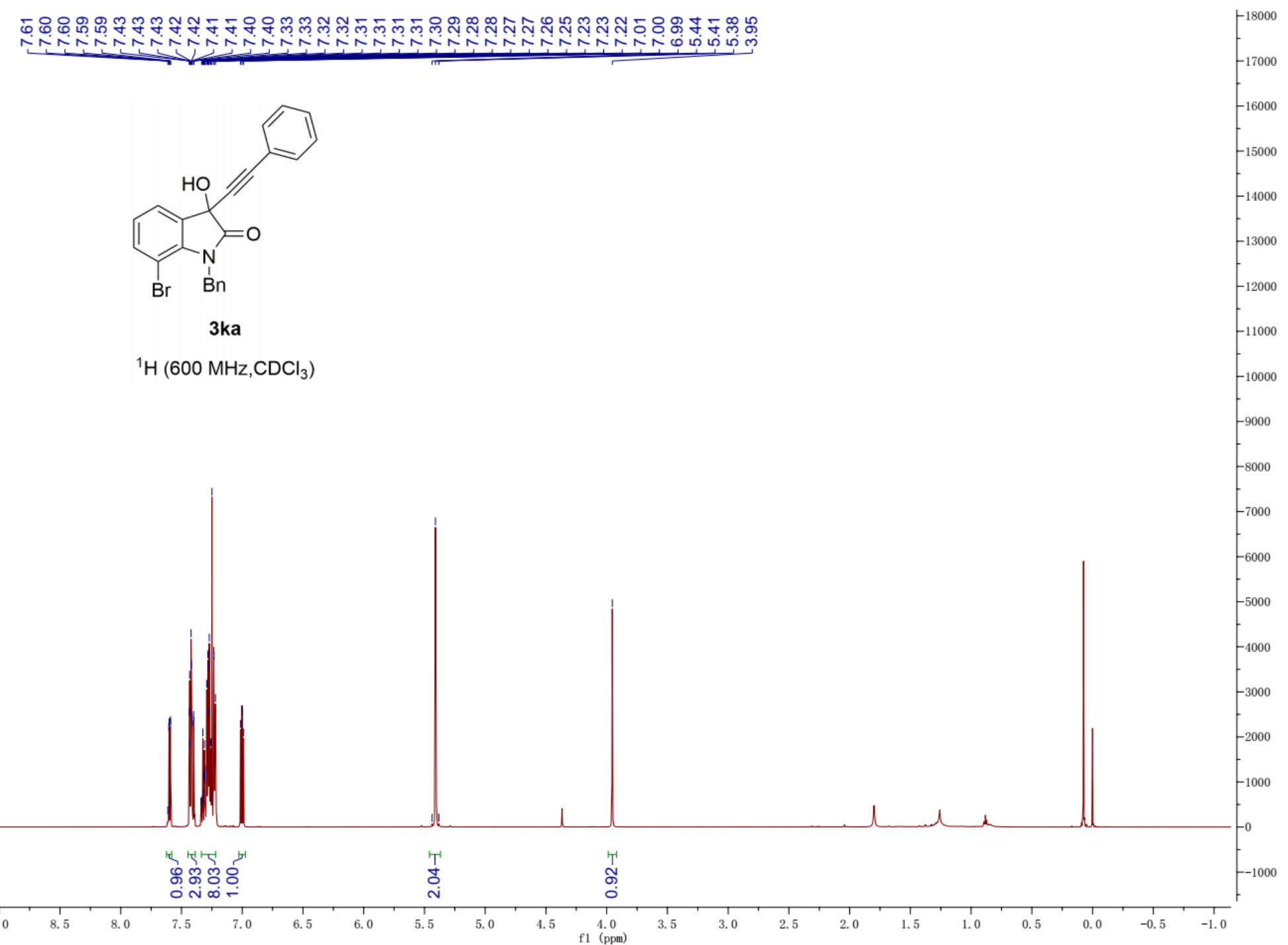




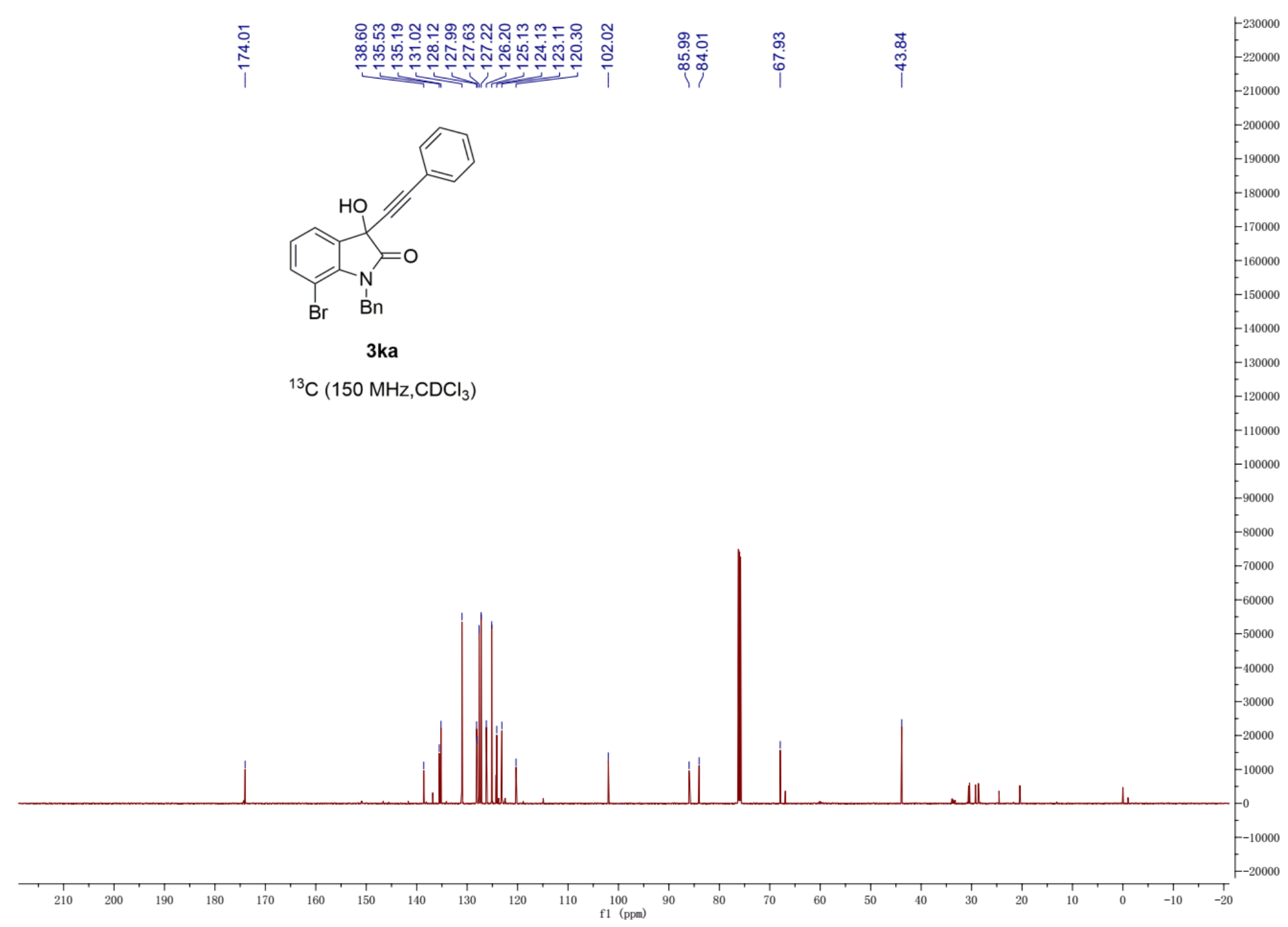

S97 


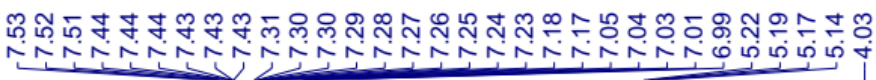

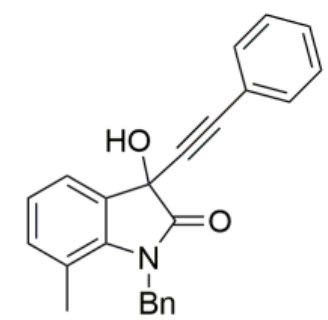

3la

${ }^{1} \mathrm{H}\left(600 \mathrm{MHz}, \mathrm{CDCl}_{3}\right)$

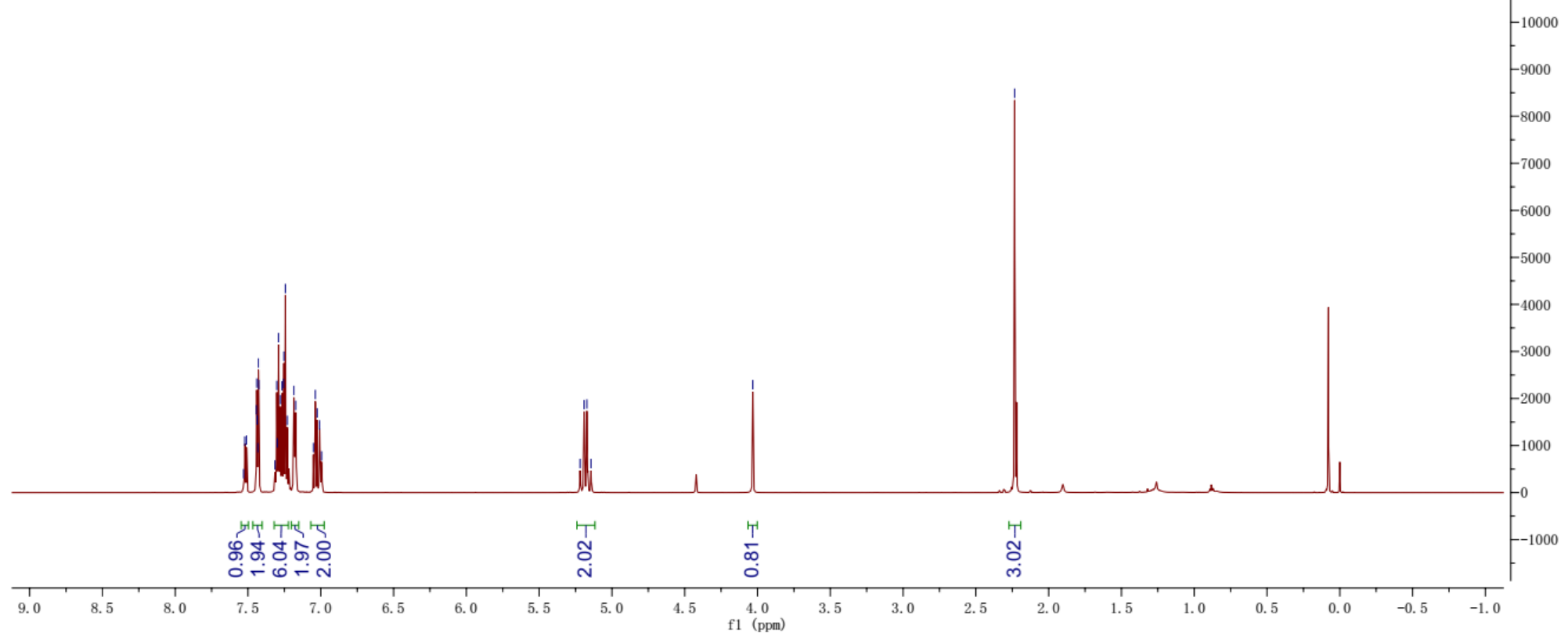




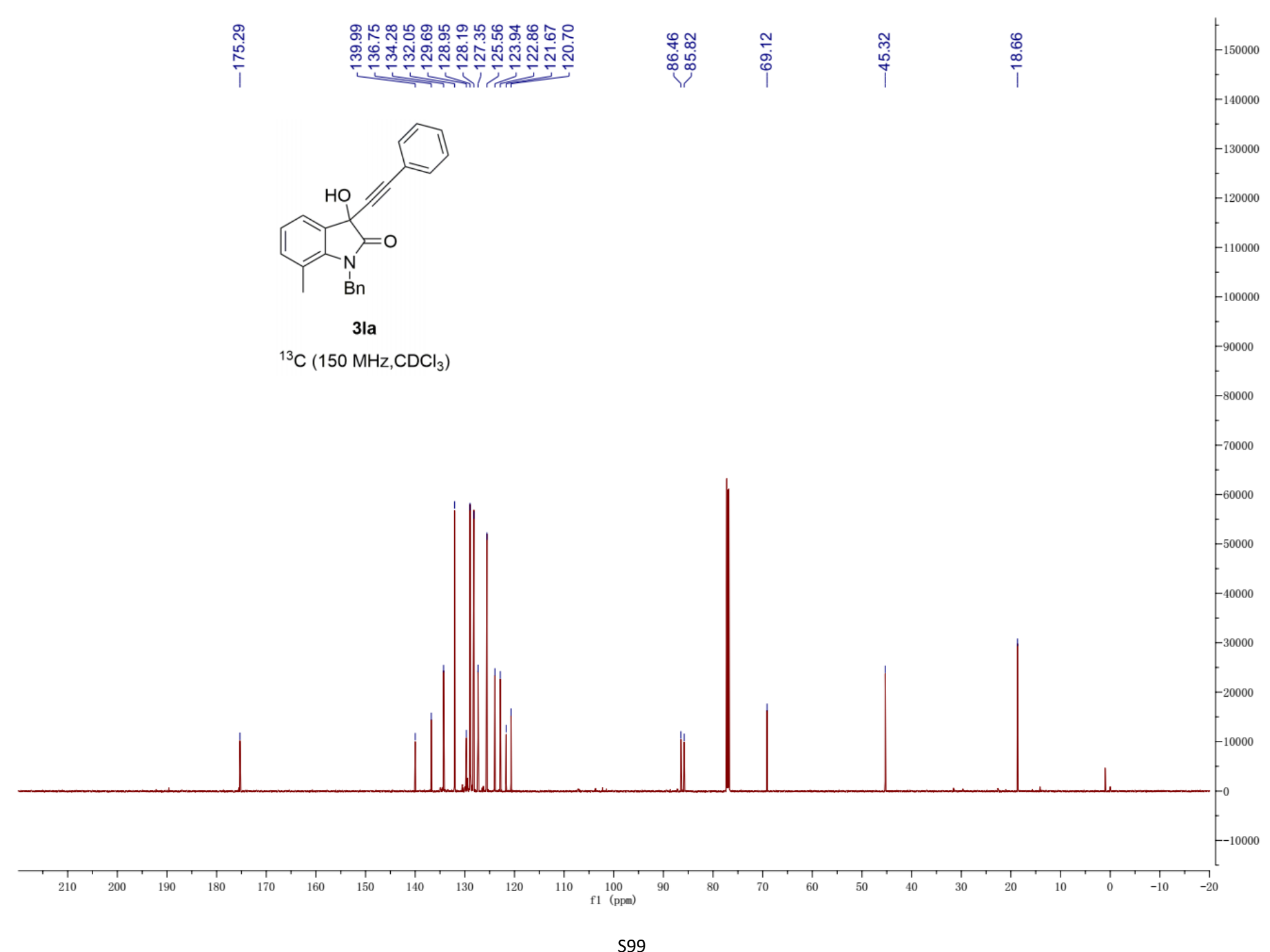




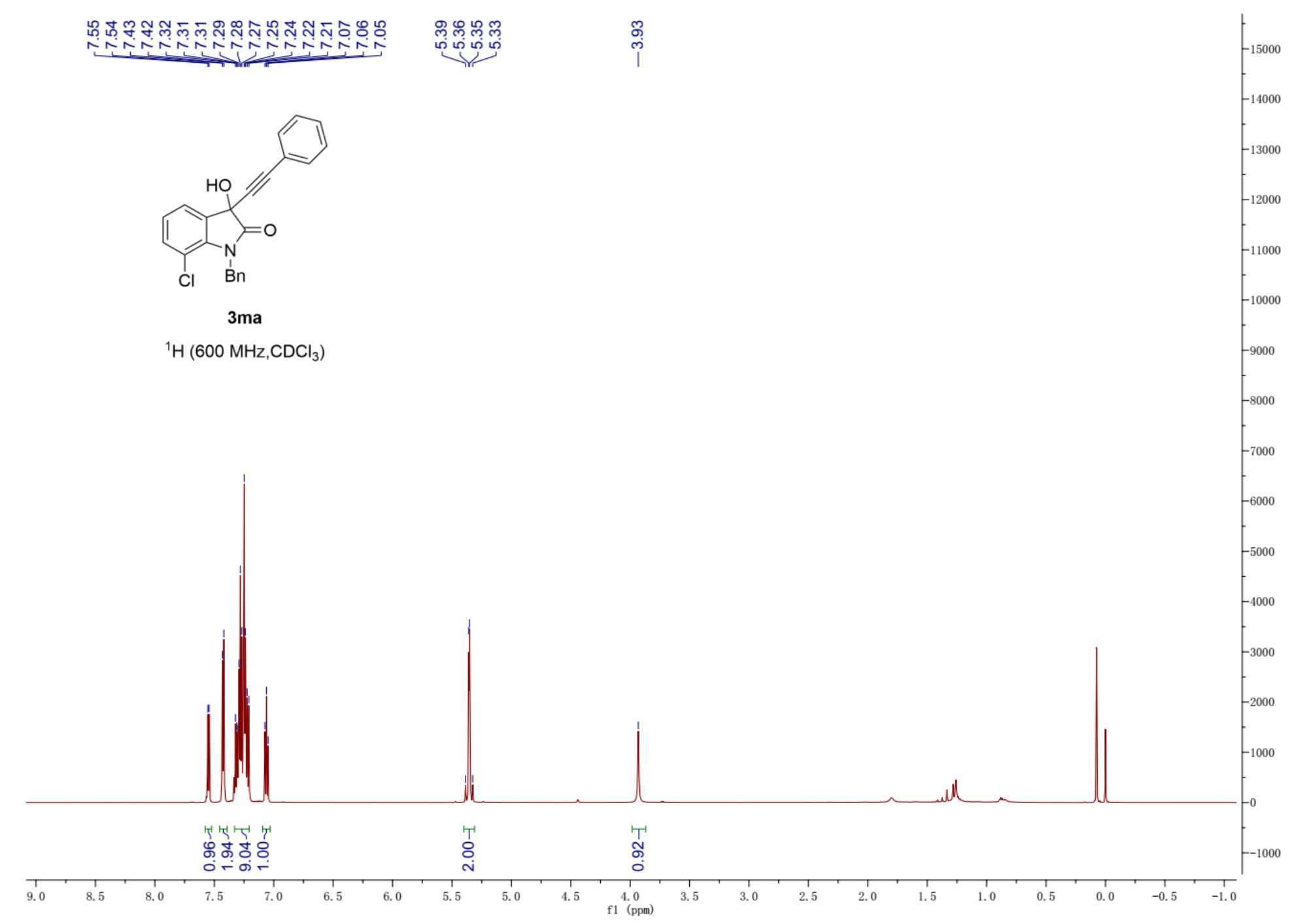




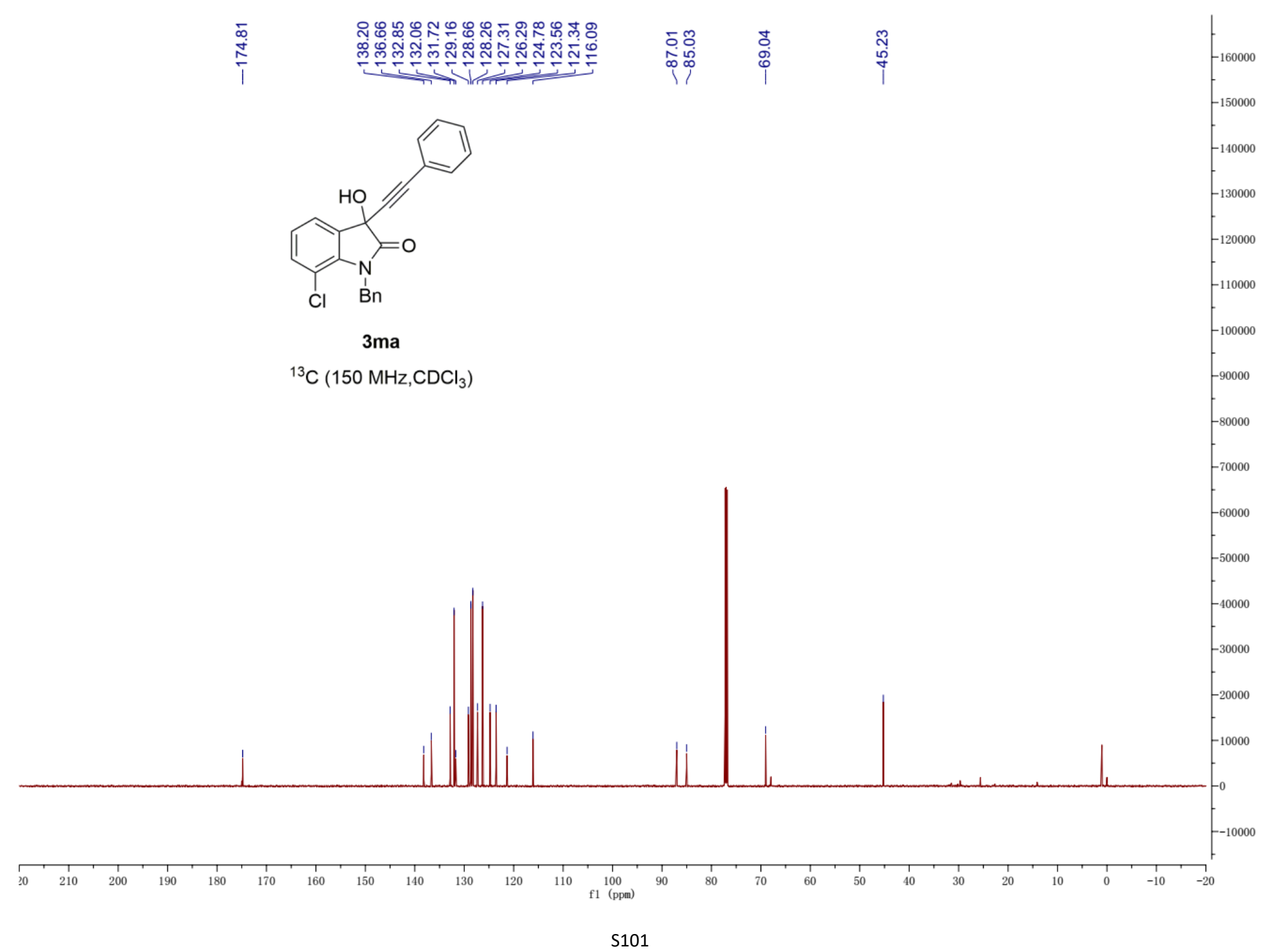




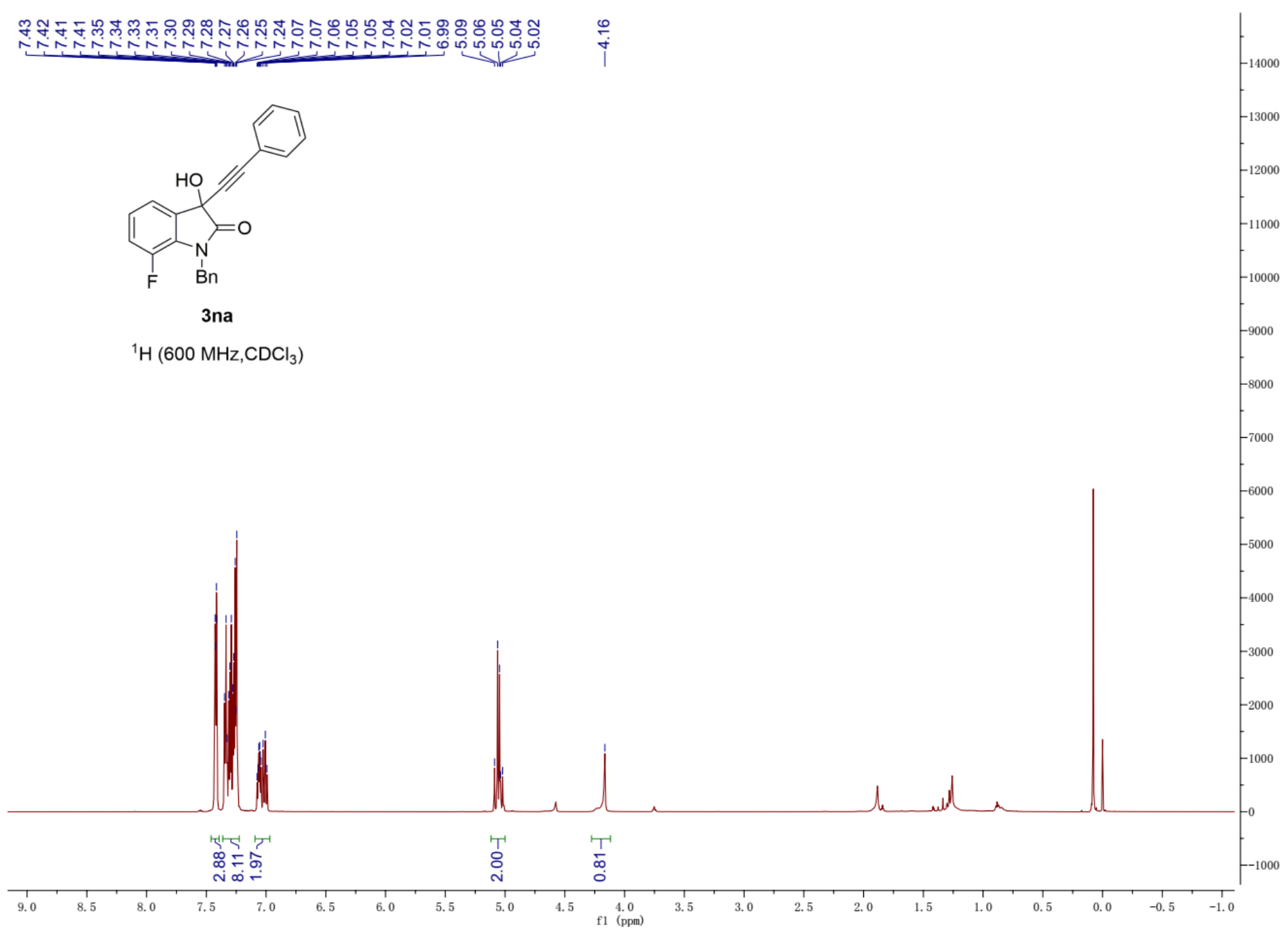




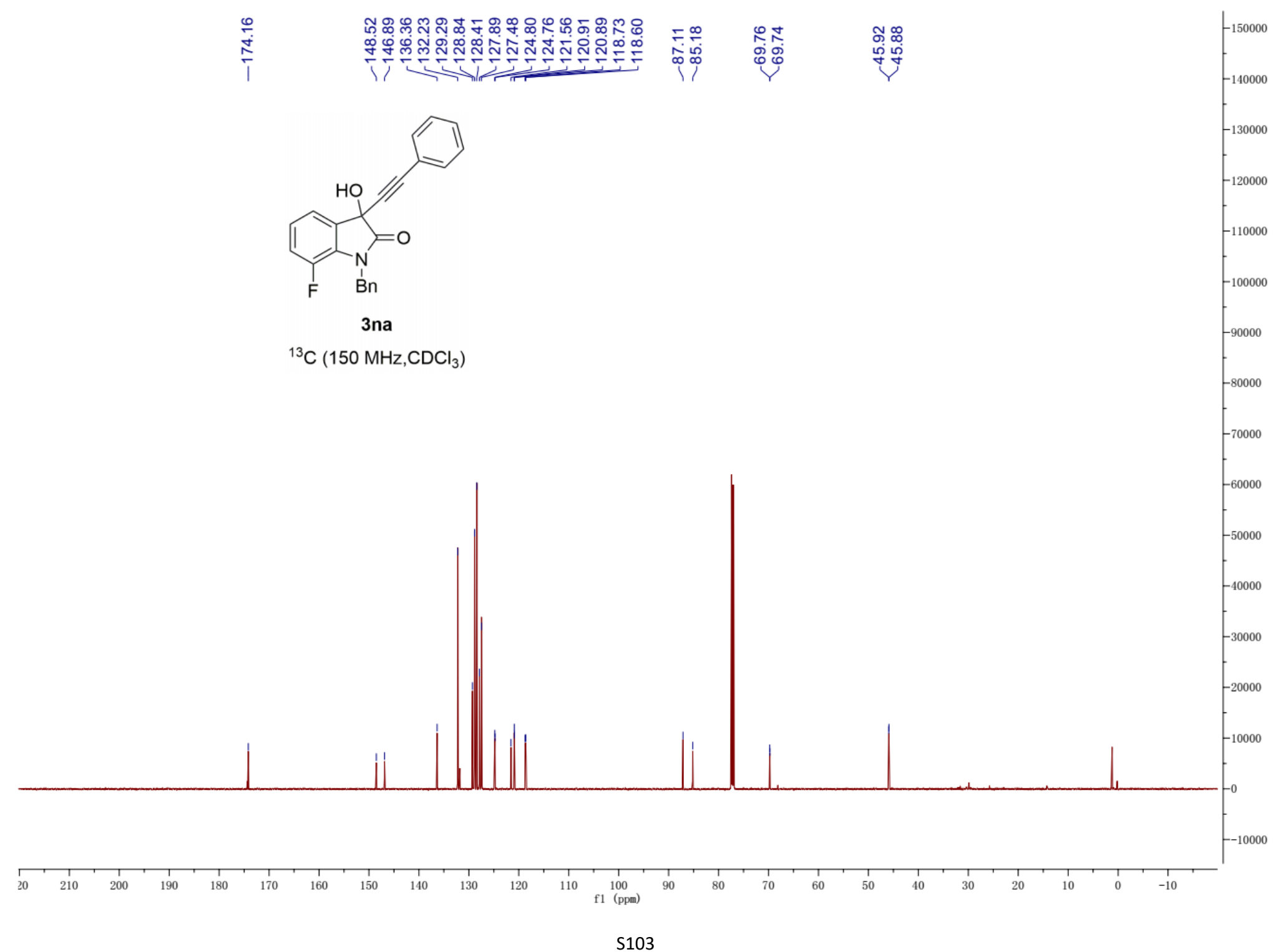




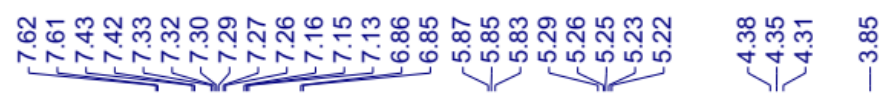

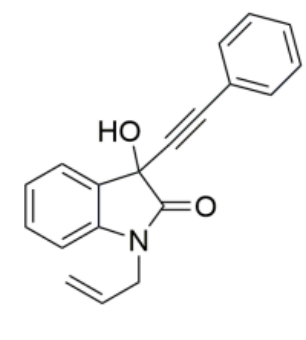

3oa

${ }^{1} \mathrm{H}\left(600 \mathrm{MHz}, \mathrm{CDCl}_{3}\right)$

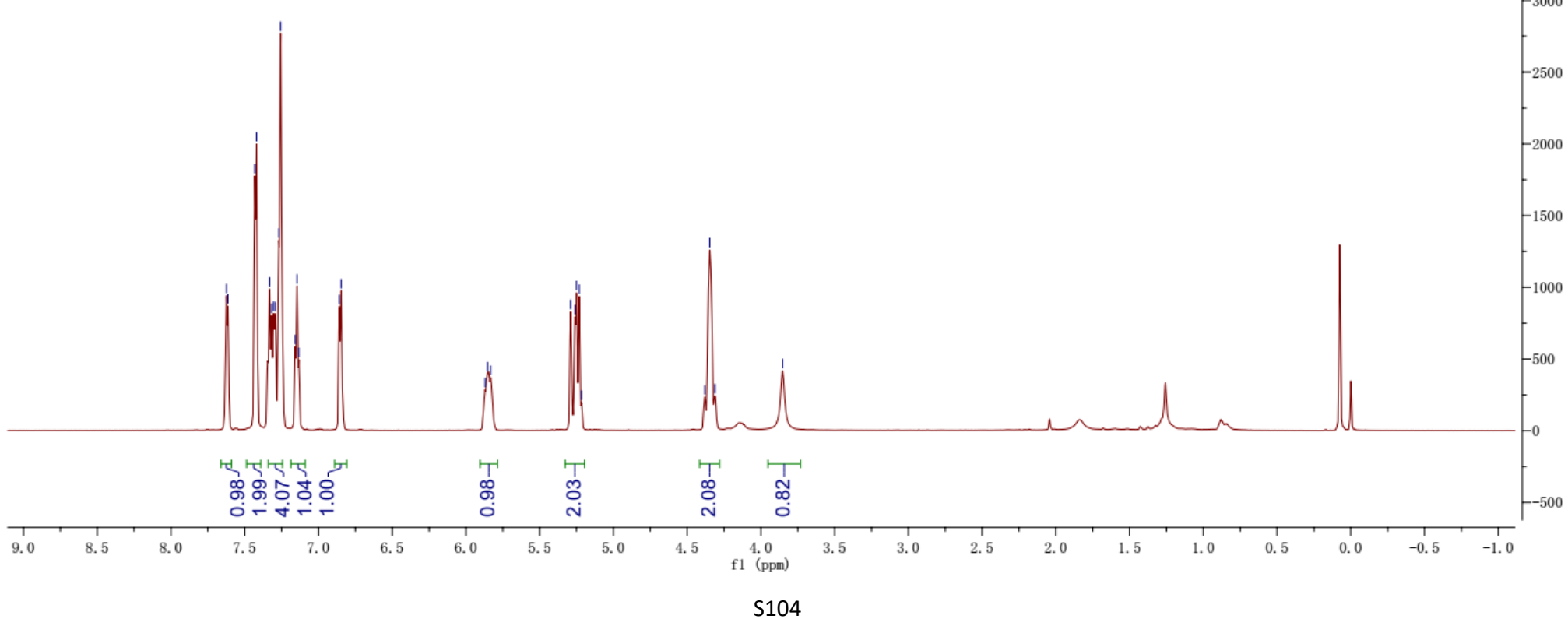




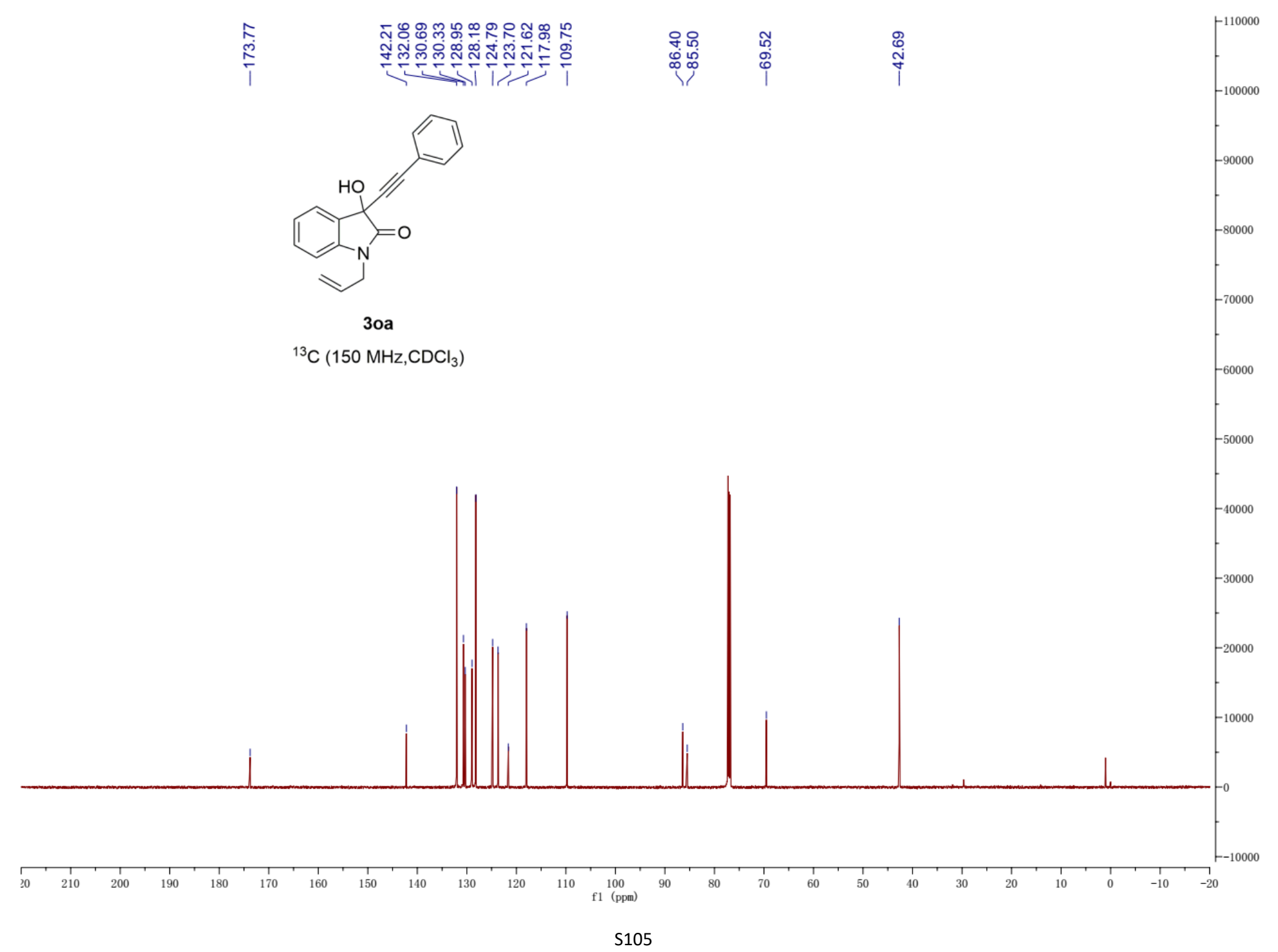




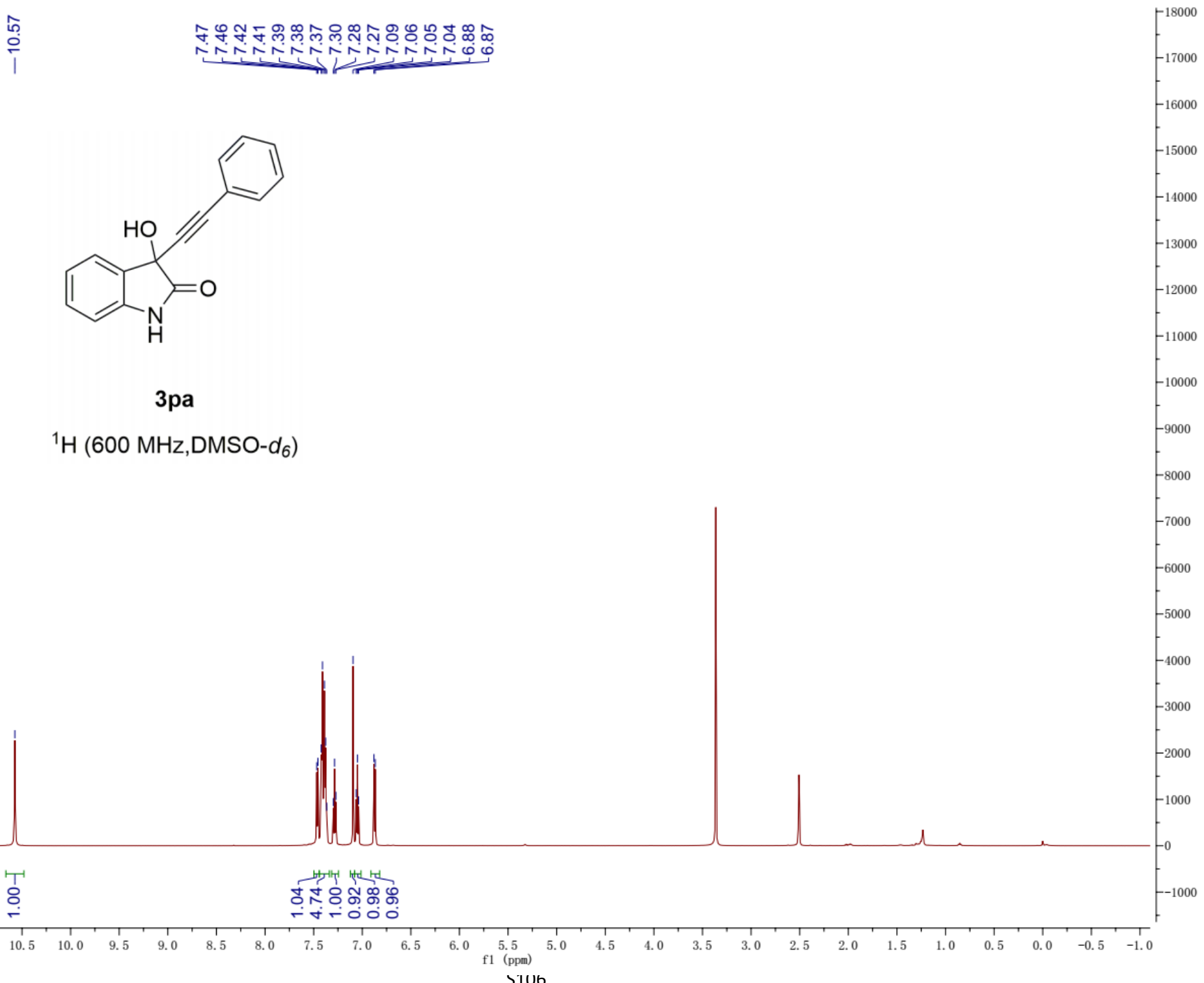




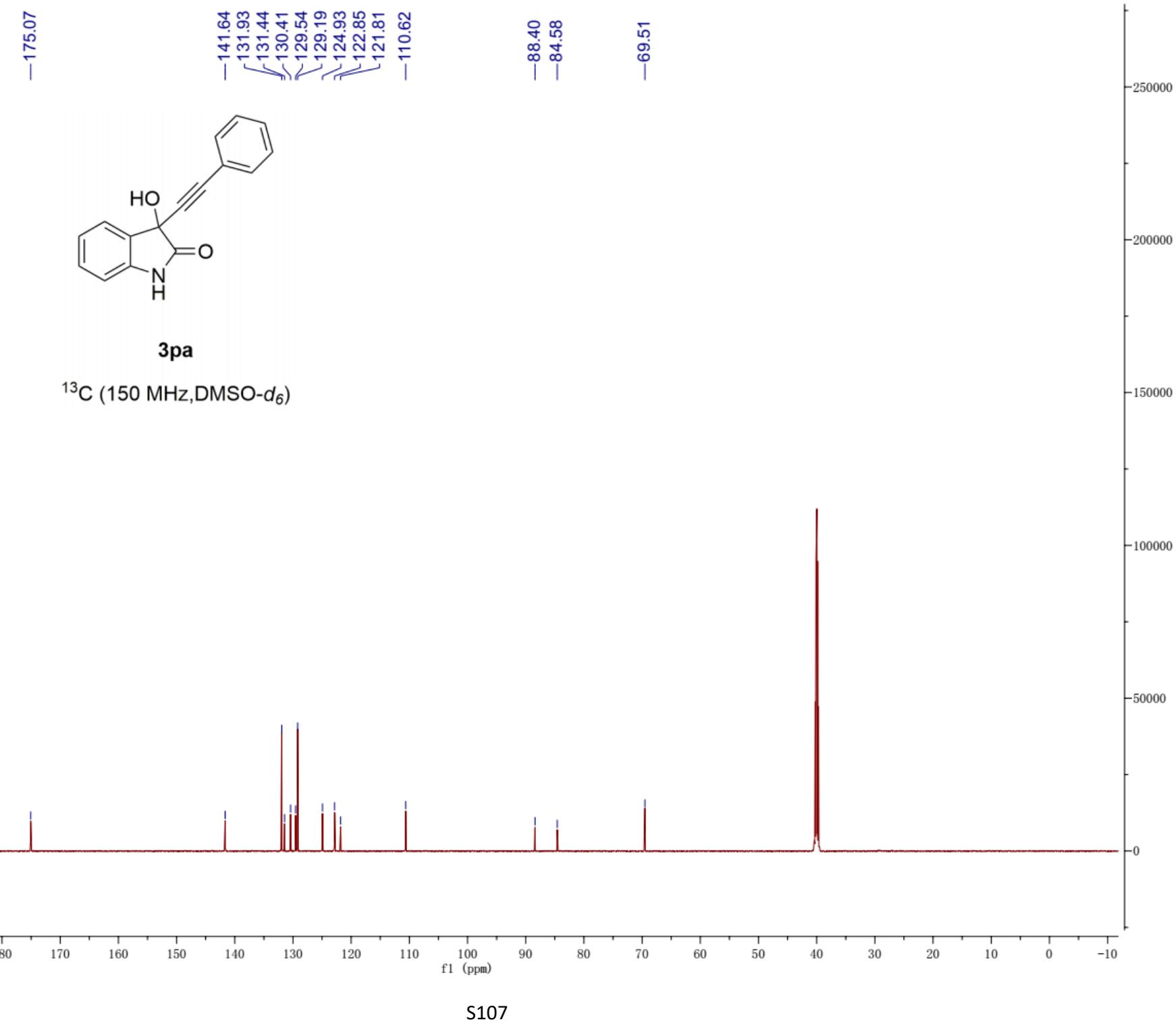




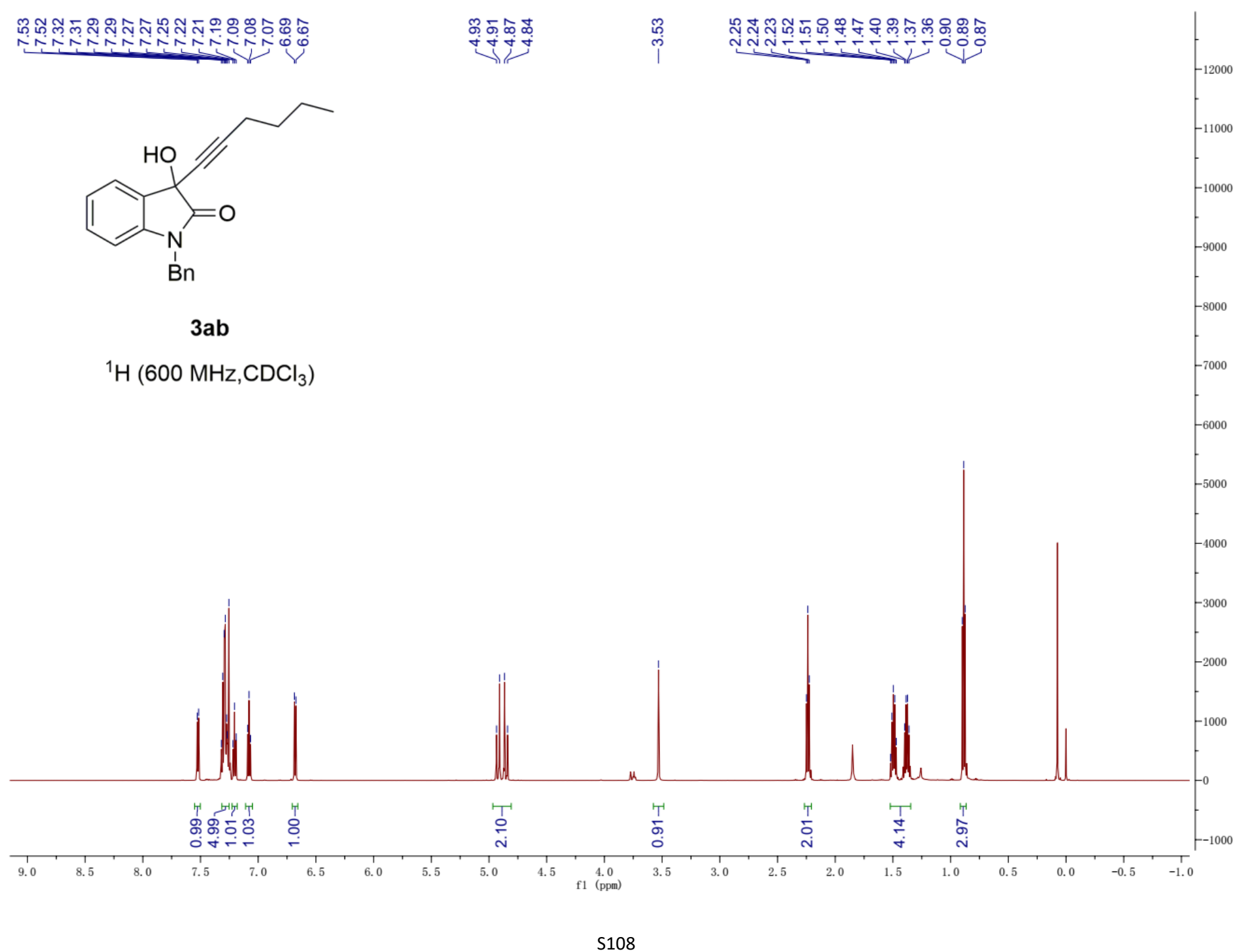




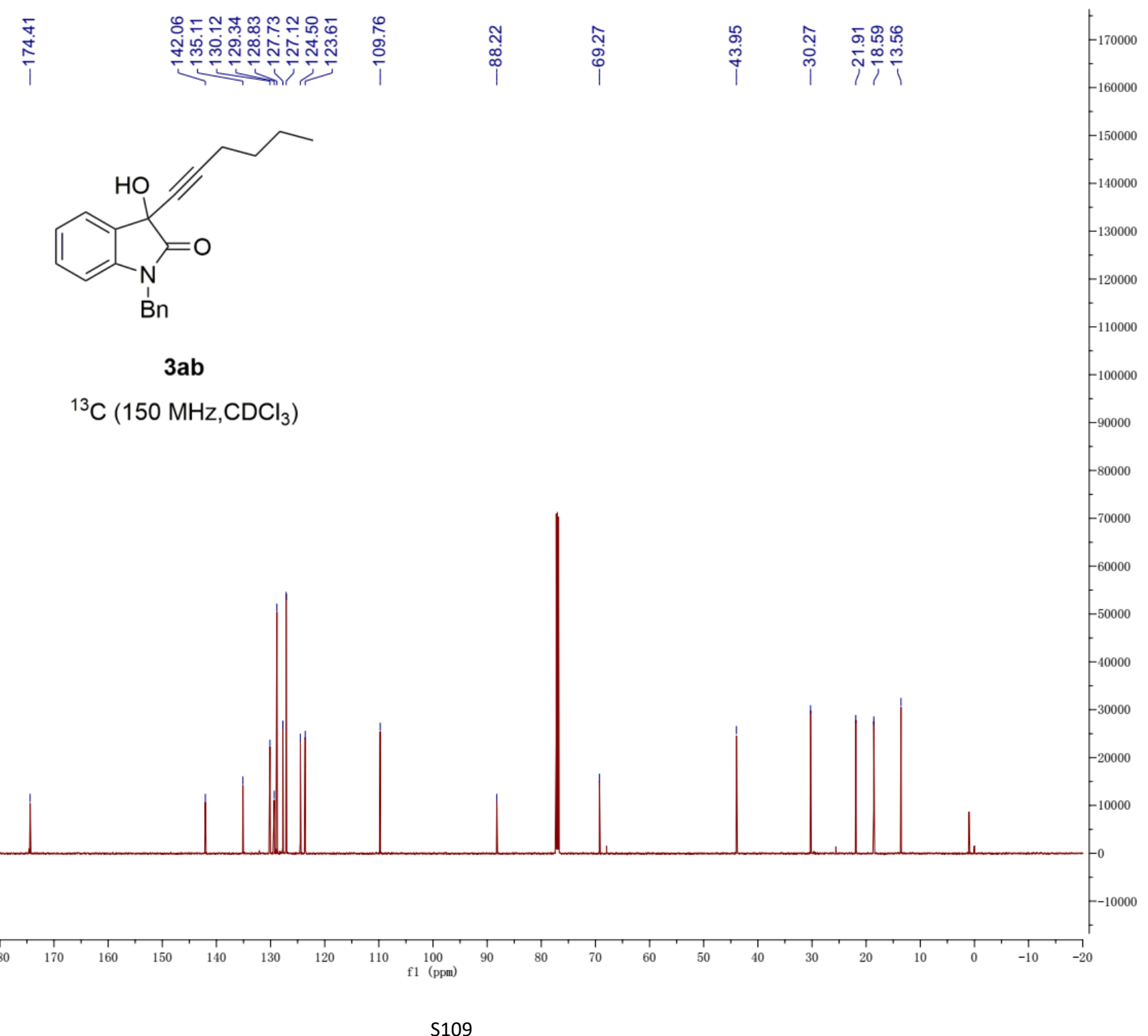




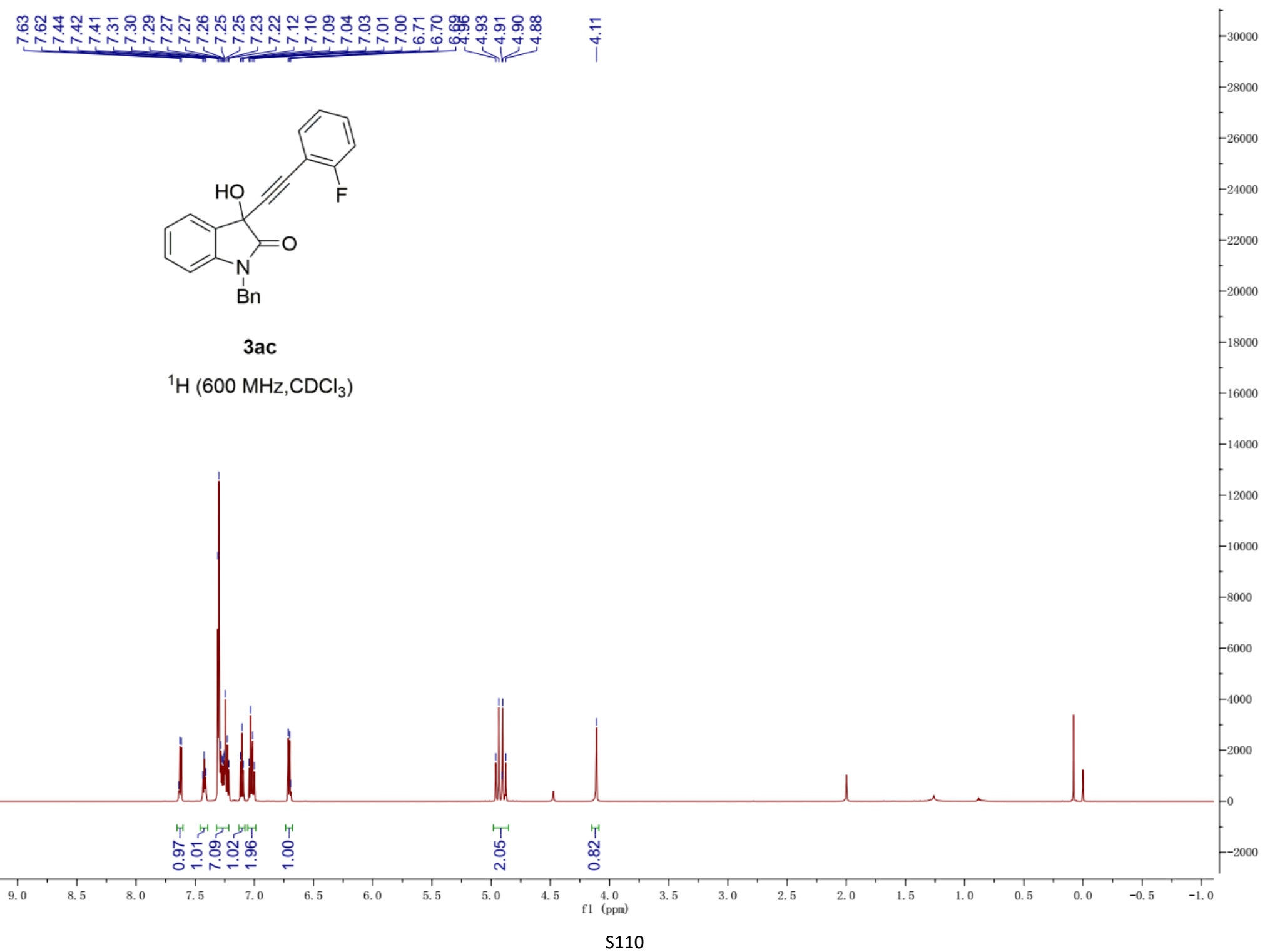




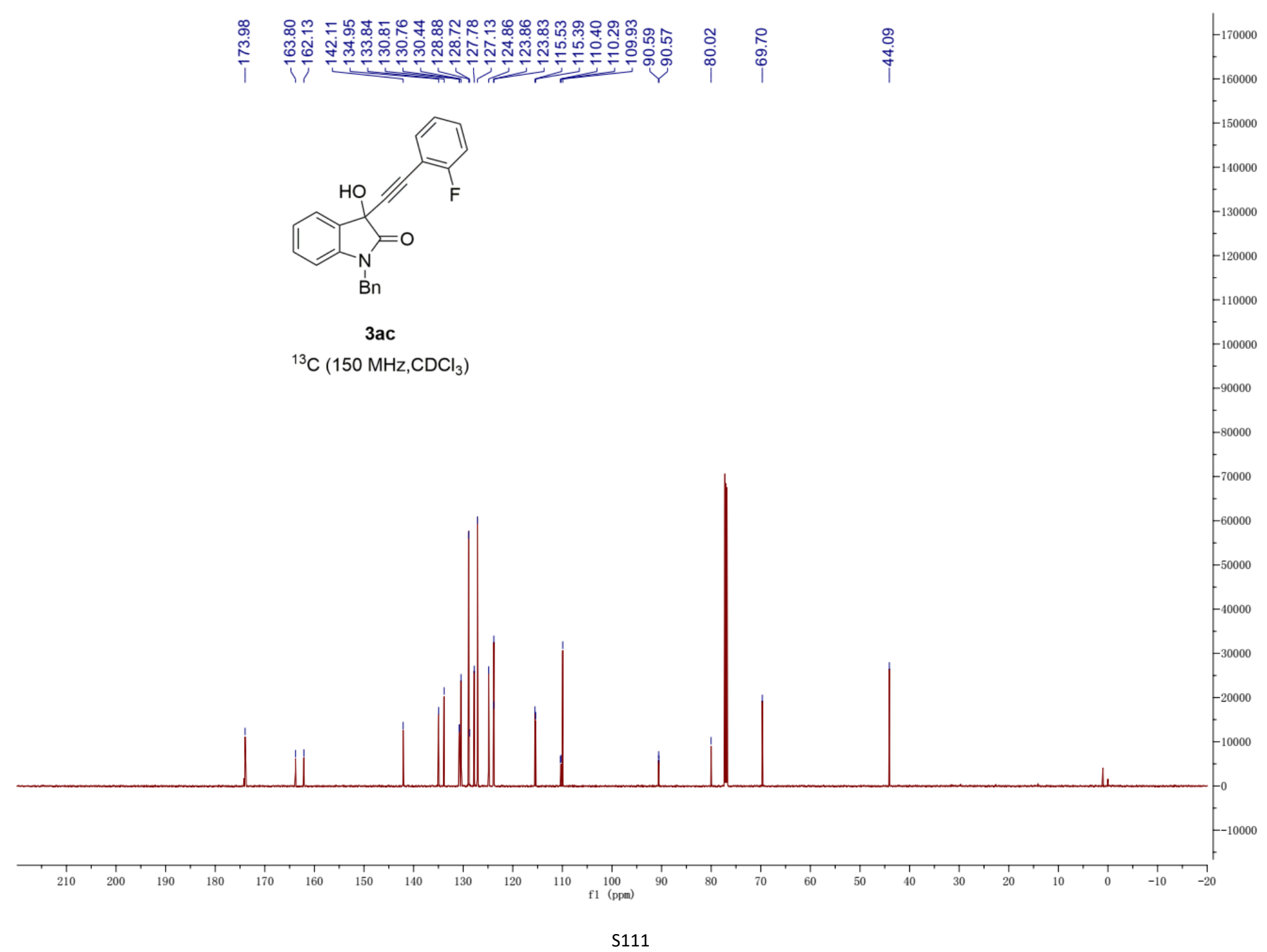




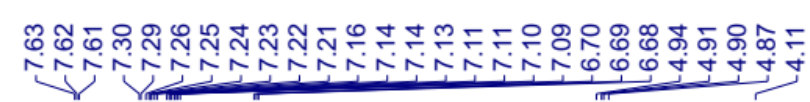

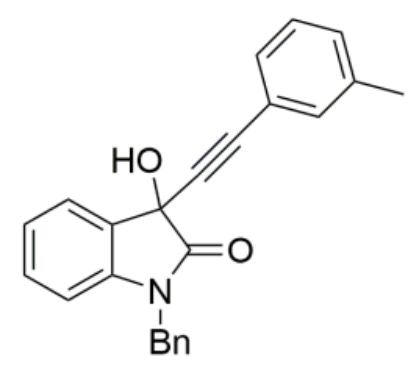

3ad

${ }^{1} \mathrm{H}\left(600 \mathrm{MHz}, \mathrm{CDCl}_{3}\right)$

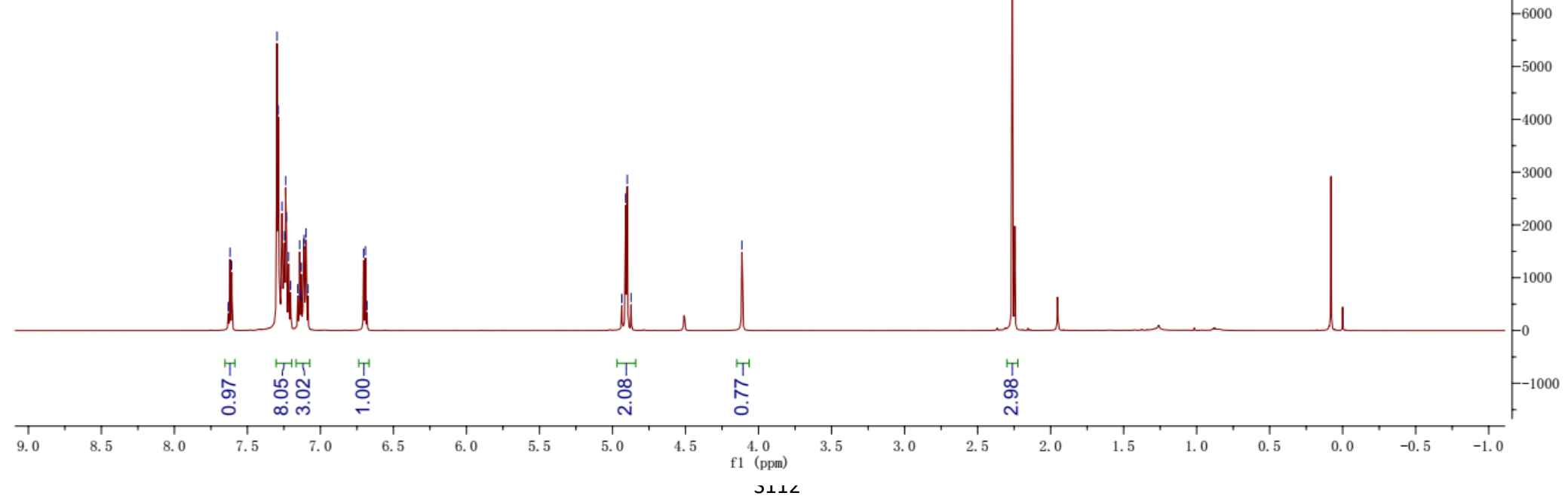




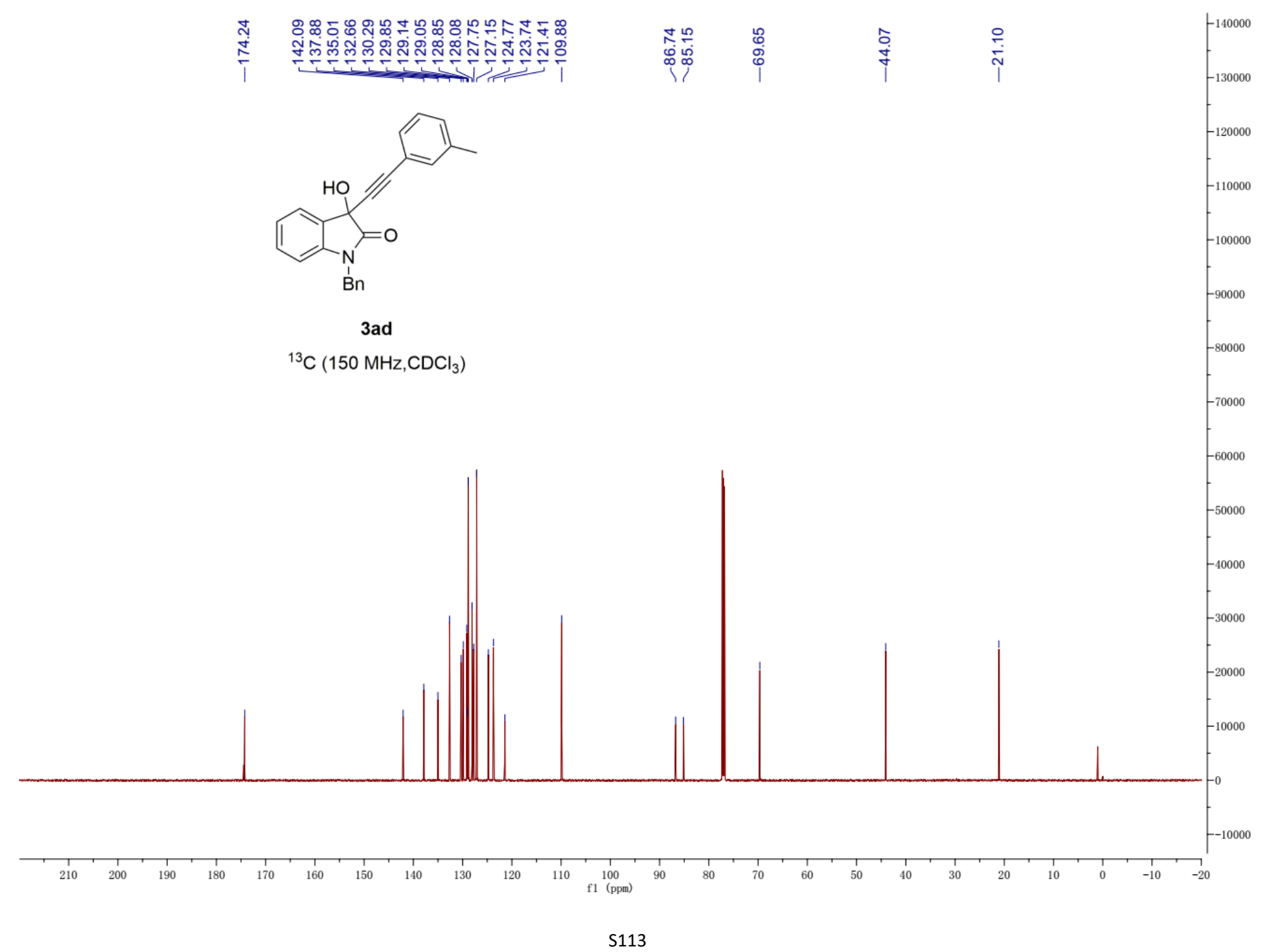




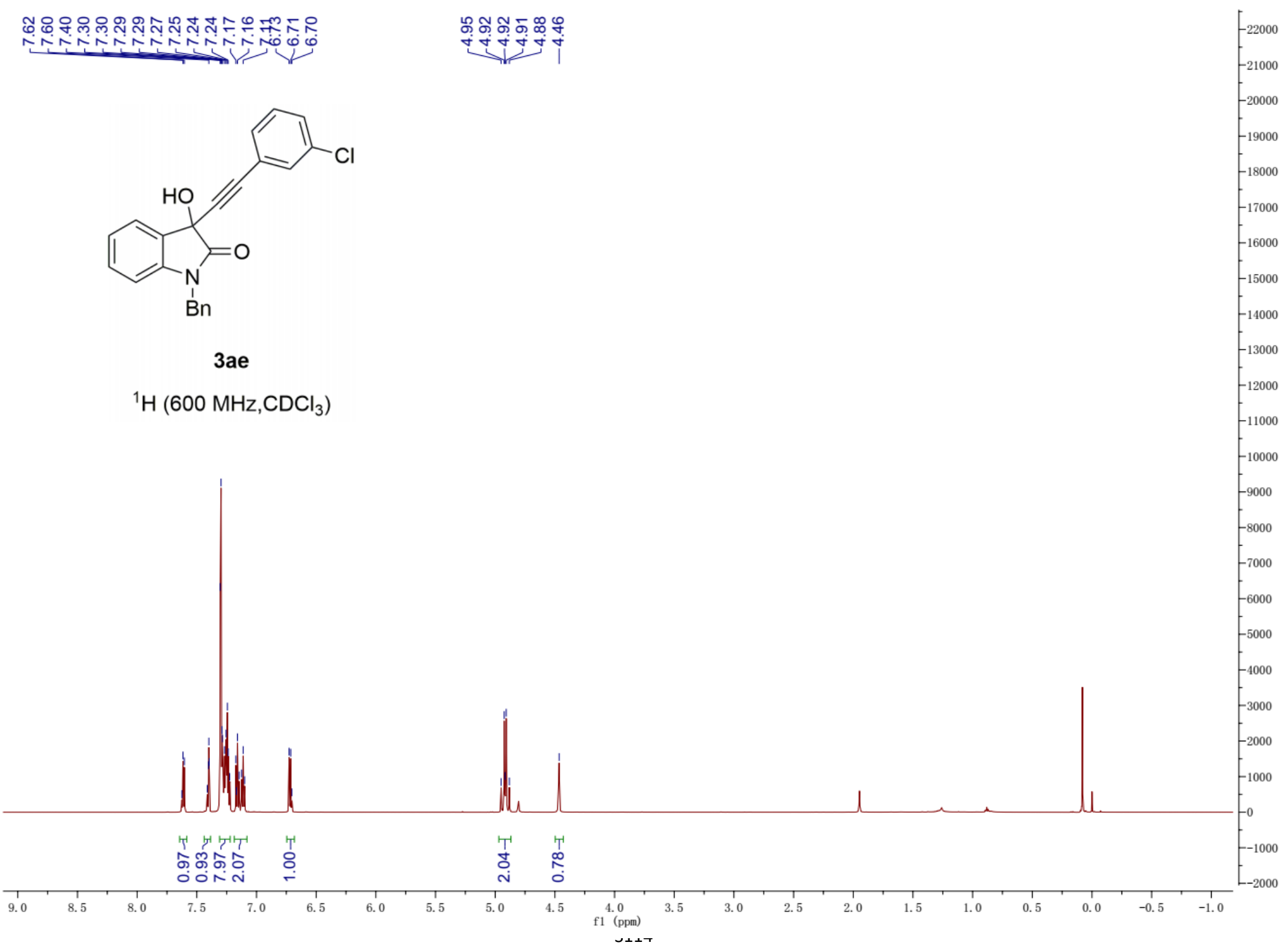




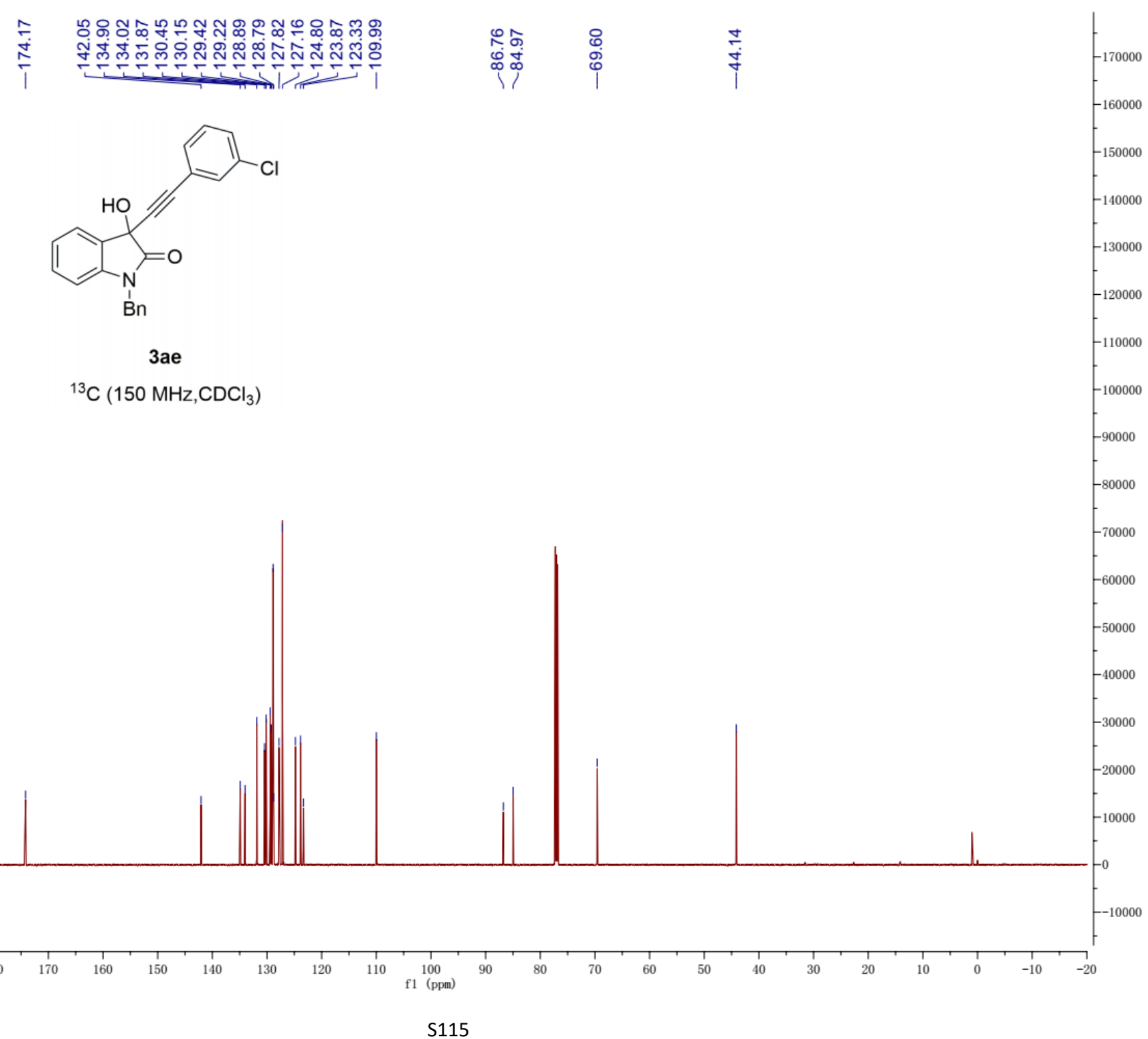




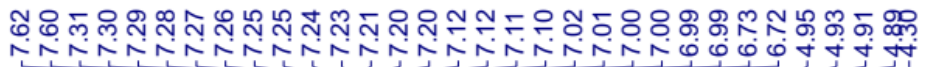

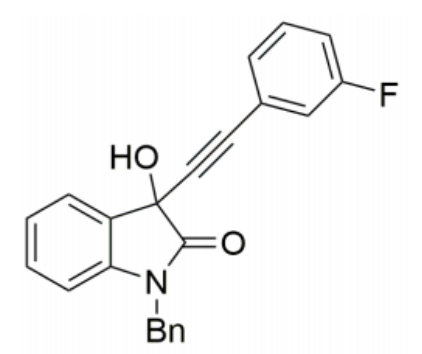

3af

${ }^{1} \mathrm{H}\left(600 \mathrm{MHz}, \mathrm{CDCl}_{3}\right)$

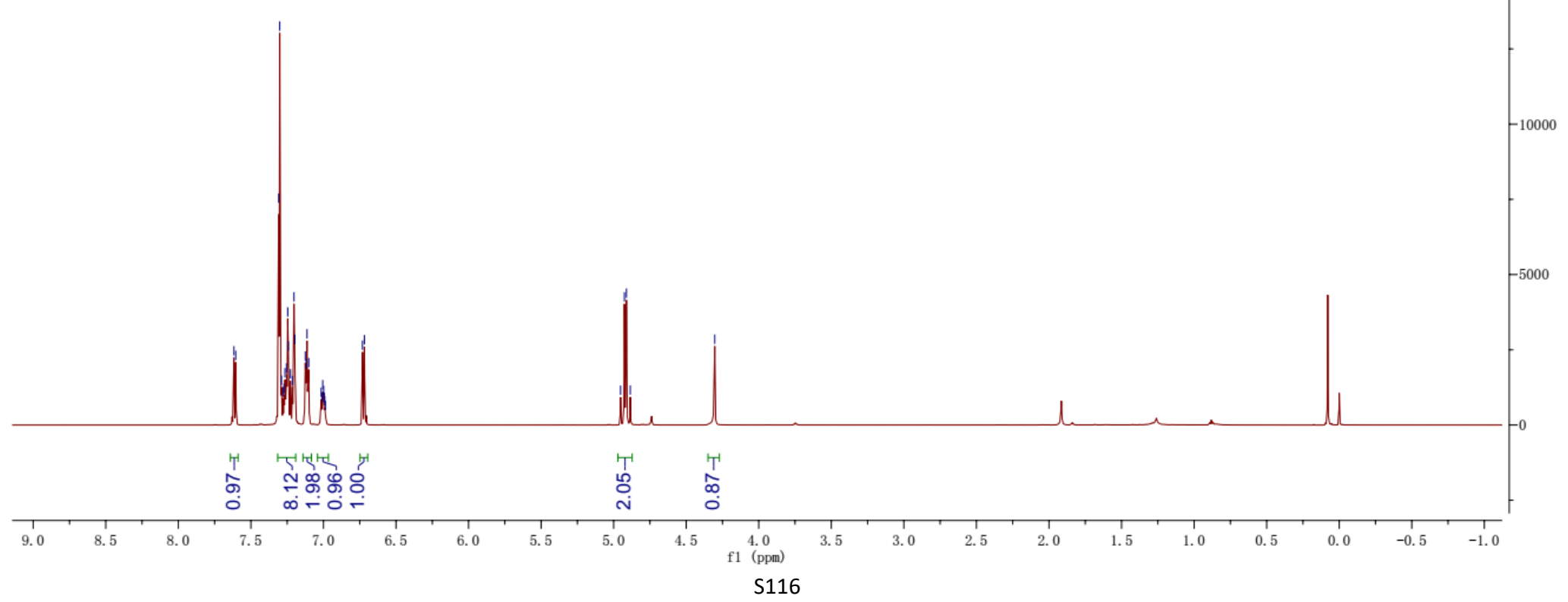




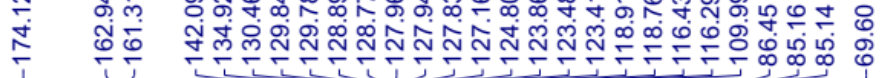

$\stackrel{+}{\stackrel{+}{+}}$

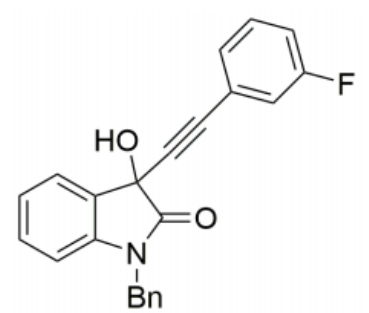

3af

${ }^{13} \mathrm{C}\left(150 \mathrm{MHz}, \mathrm{CDCl}_{3}\right)$

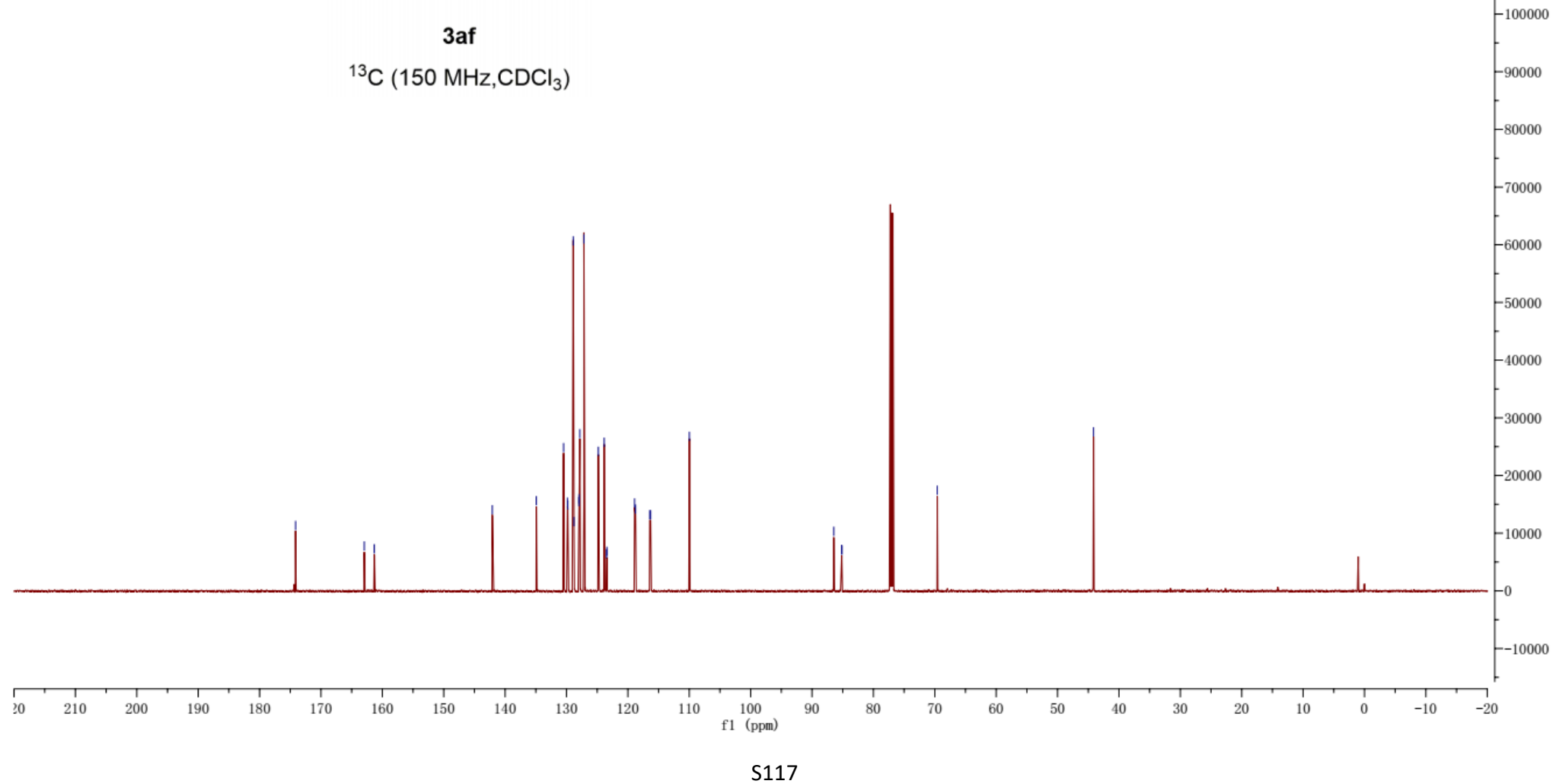




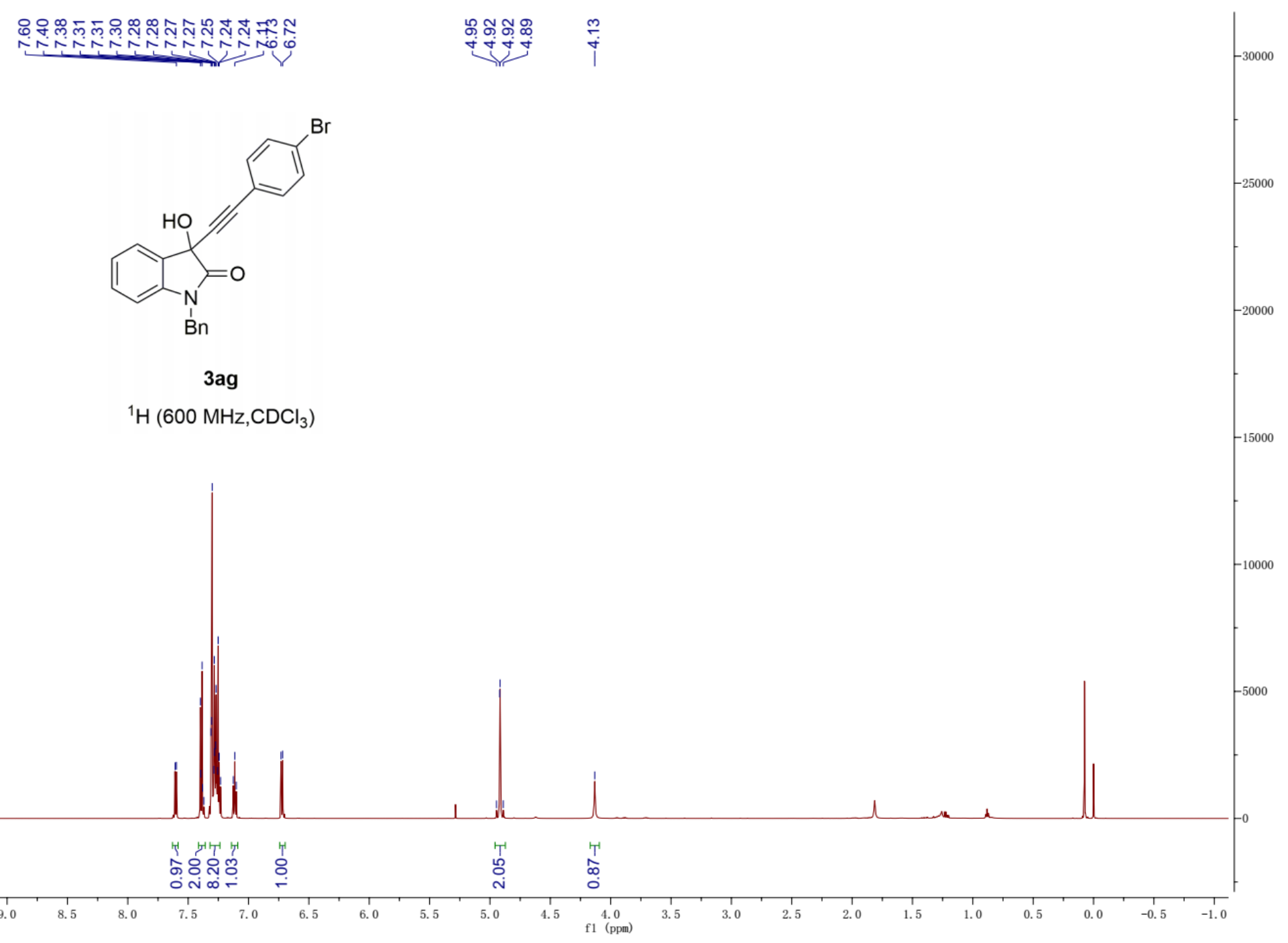




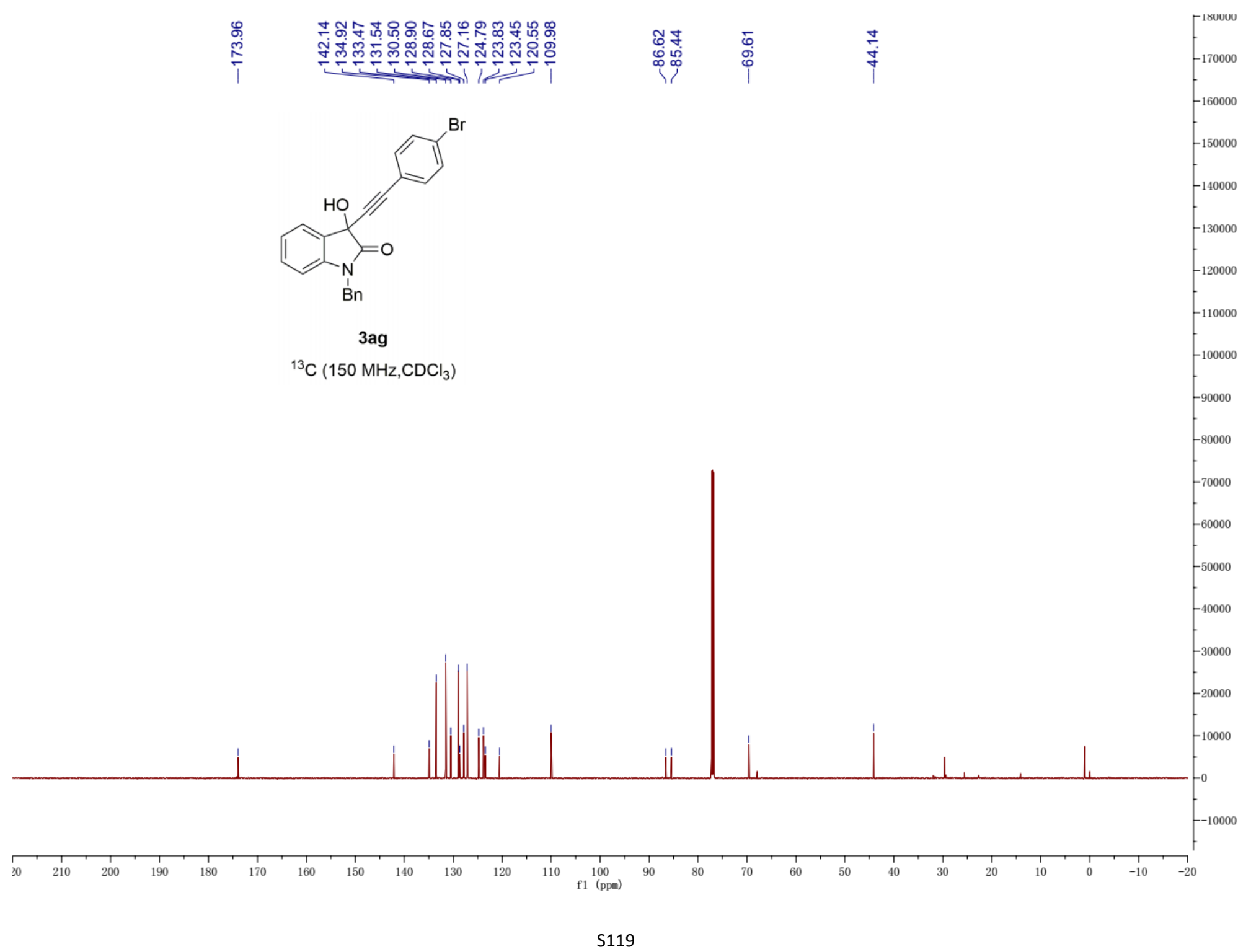




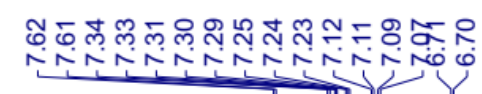

$\stackrel{\text { ๙ }}{+}$

$\stackrel{\infty}{\infty}$

$\stackrel{\mathscr{m}}{i}$

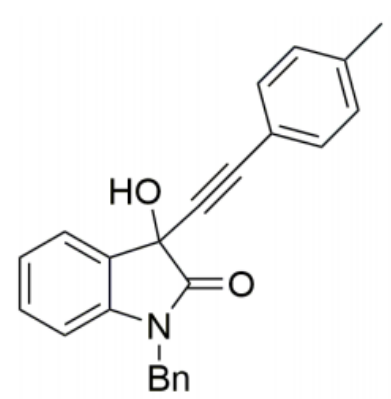

3ah

${ }^{1} \mathrm{H}\left(600 \mathrm{MHz}, \mathrm{CDCl}_{3}\right)$

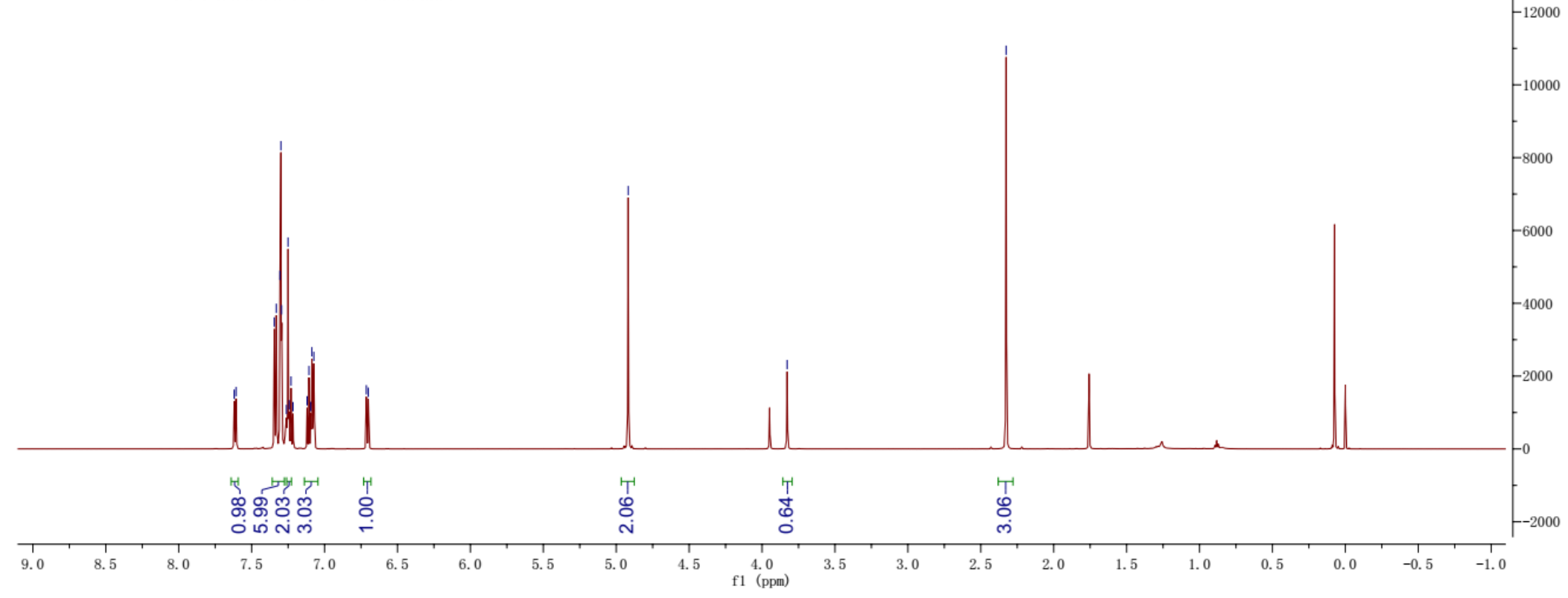




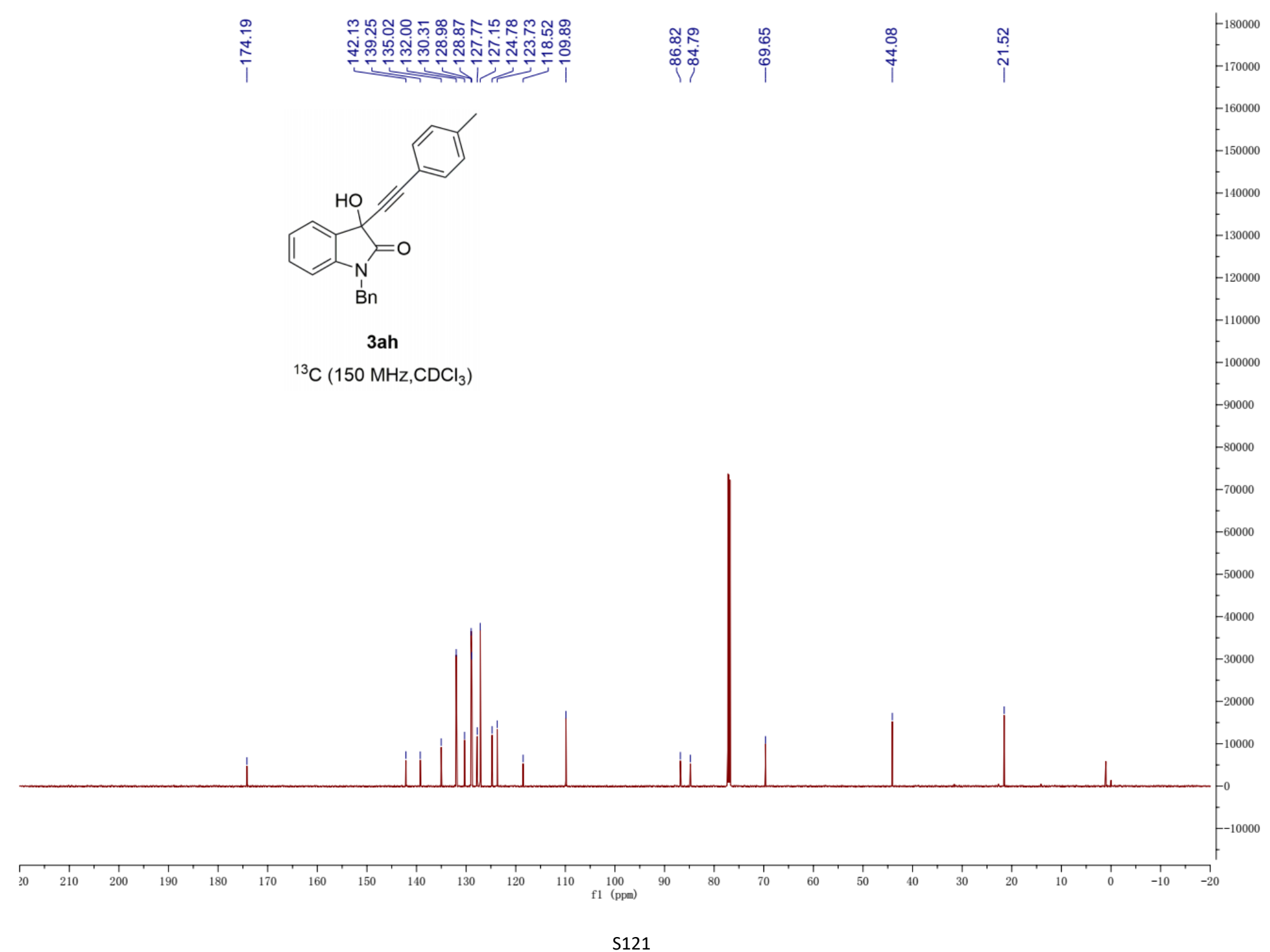


잉ำ

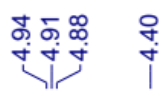

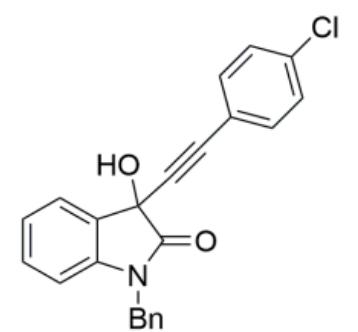

$\mathrm{Bn}$

$3 a i$

${ }^{1} \mathrm{H}\left(600 \mathrm{MHz}, \mathrm{CDCl}_{3}\right)$

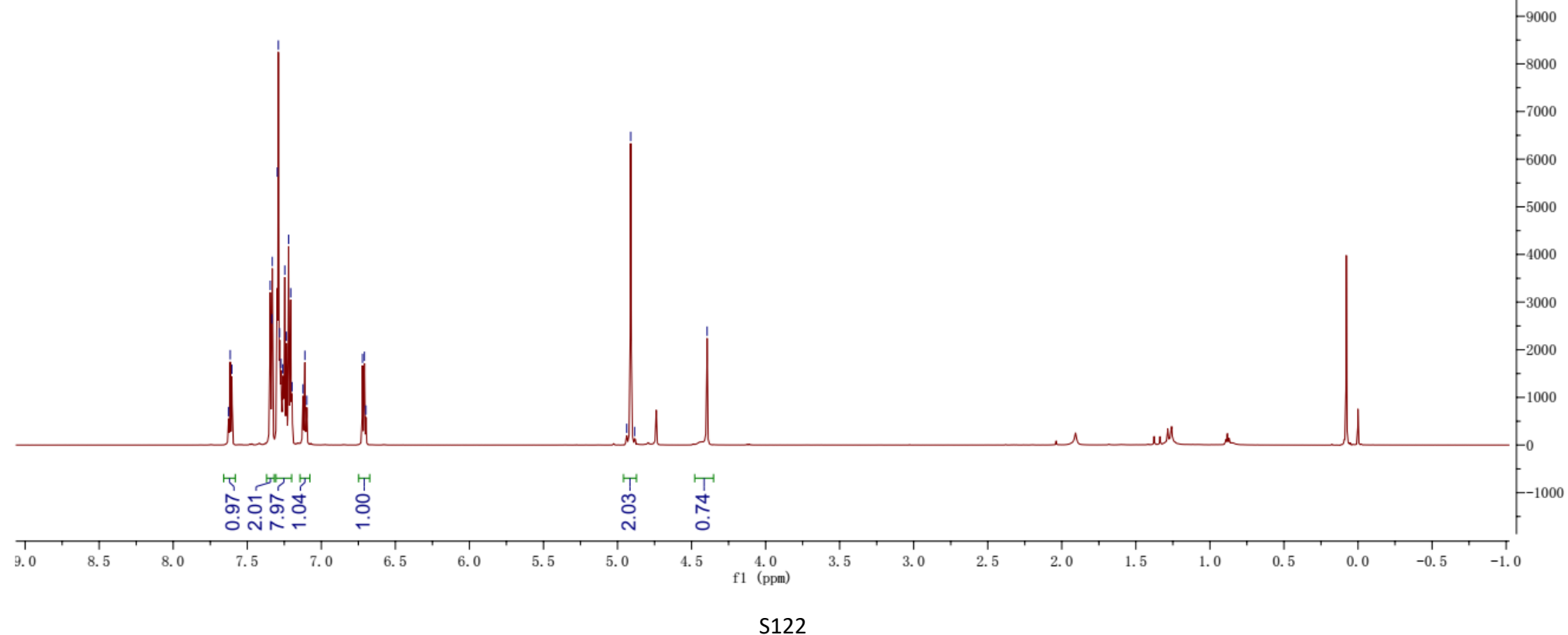




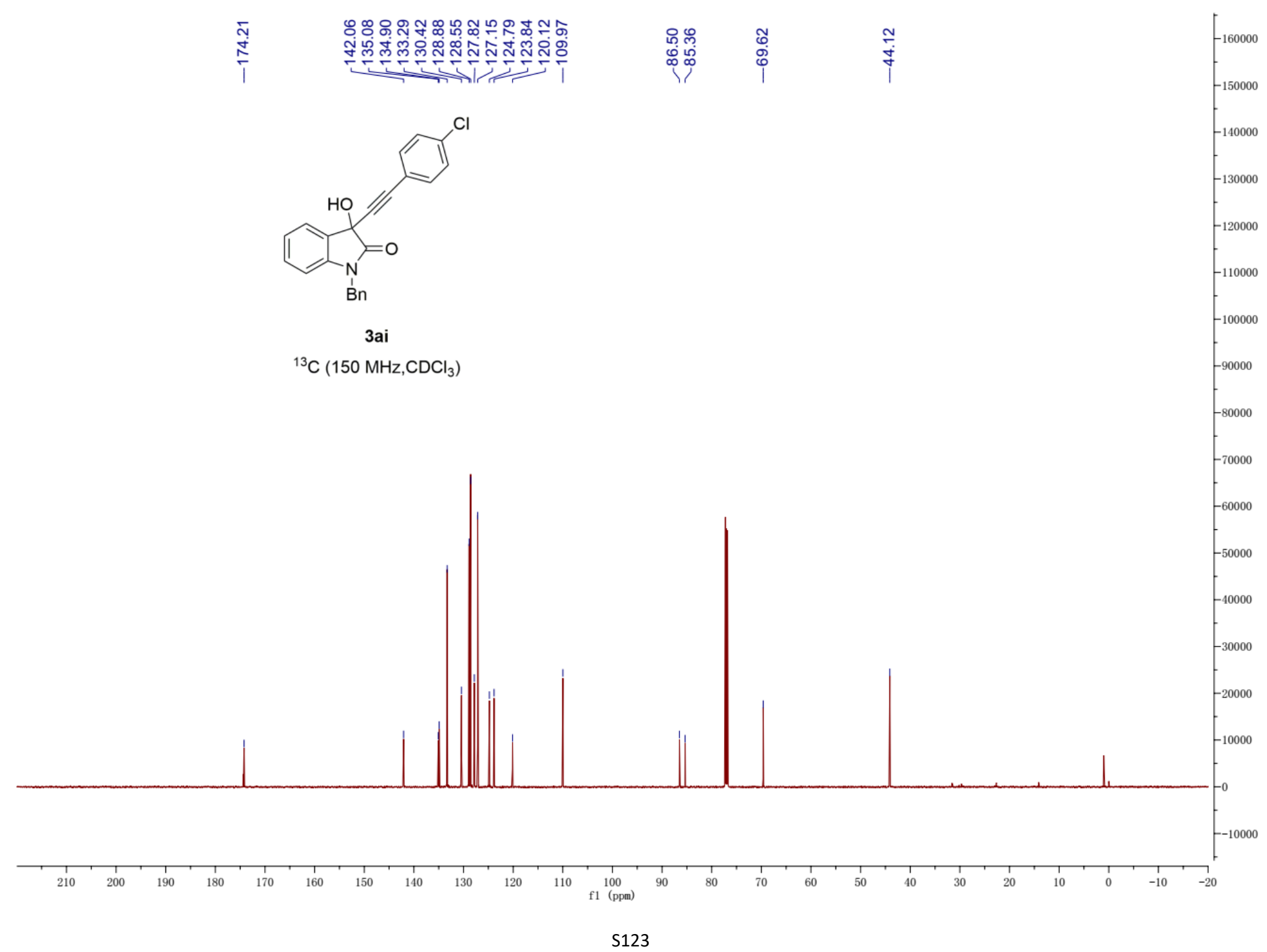




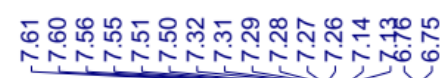

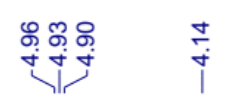

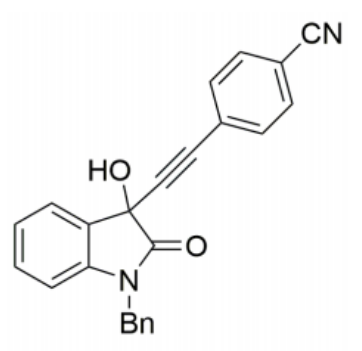

3aj

${ }^{1} \mathrm{H}\left(600 \mathrm{MHz}, \mathrm{CDCl}_{3}\right)$

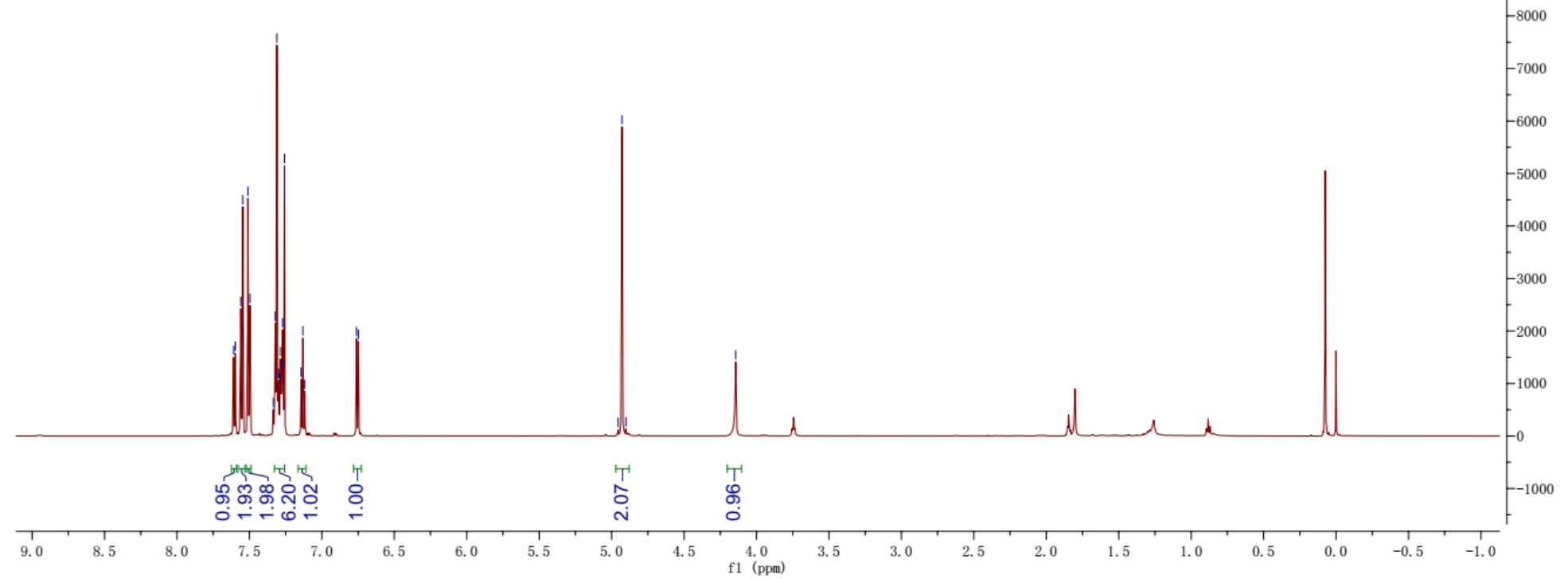




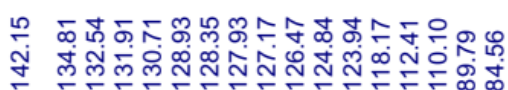

:

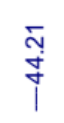

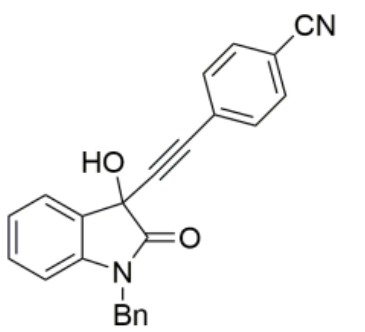

3aj

${ }^{13} \mathrm{C}\left(150 \mathrm{MHz}, \mathrm{CDCl}_{3}\right)$

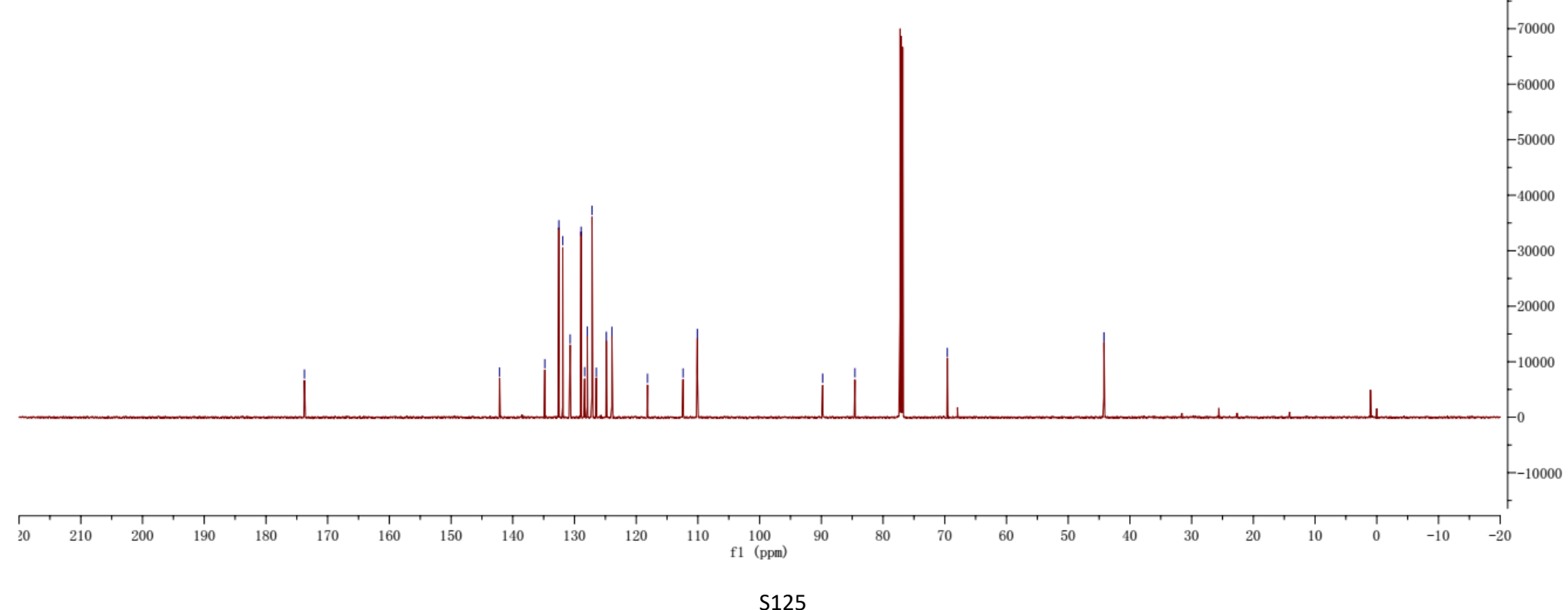




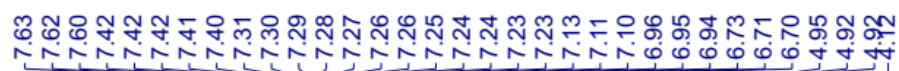

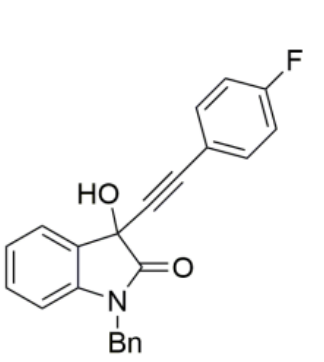

3ak

${ }^{1} \mathrm{H}\left(600 \mathrm{MHz}, \mathrm{CDCl}_{3}\right)$

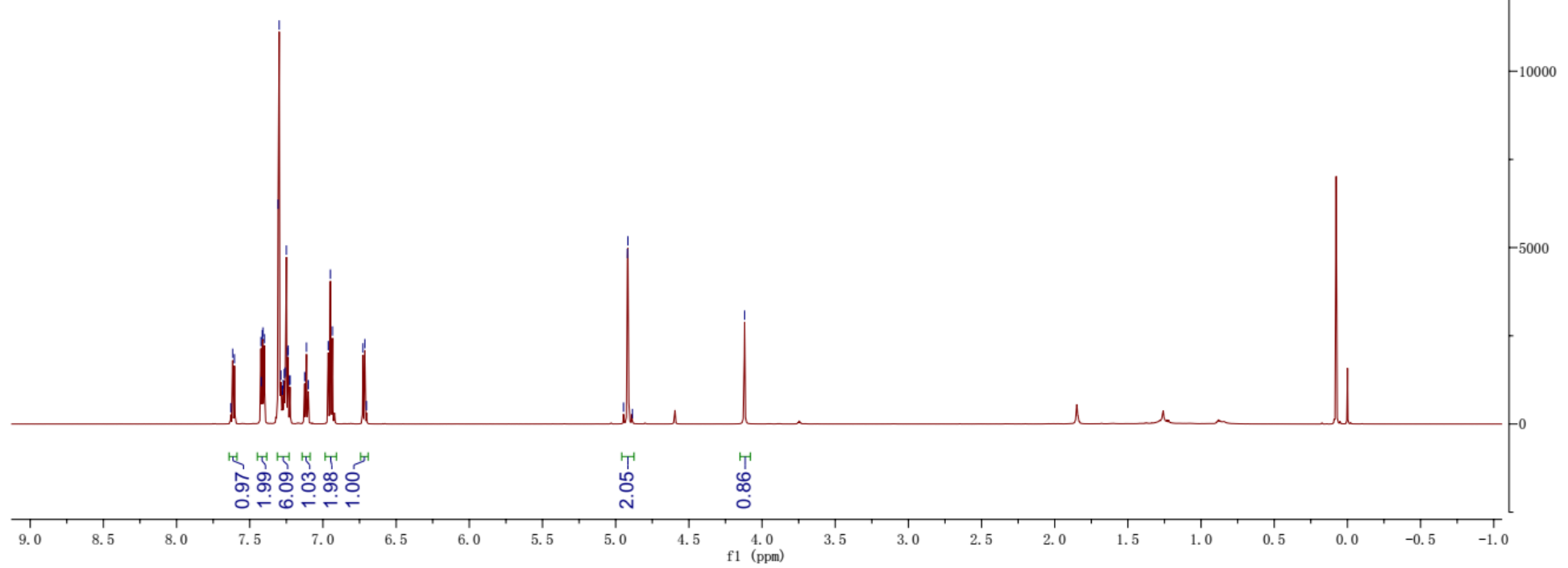




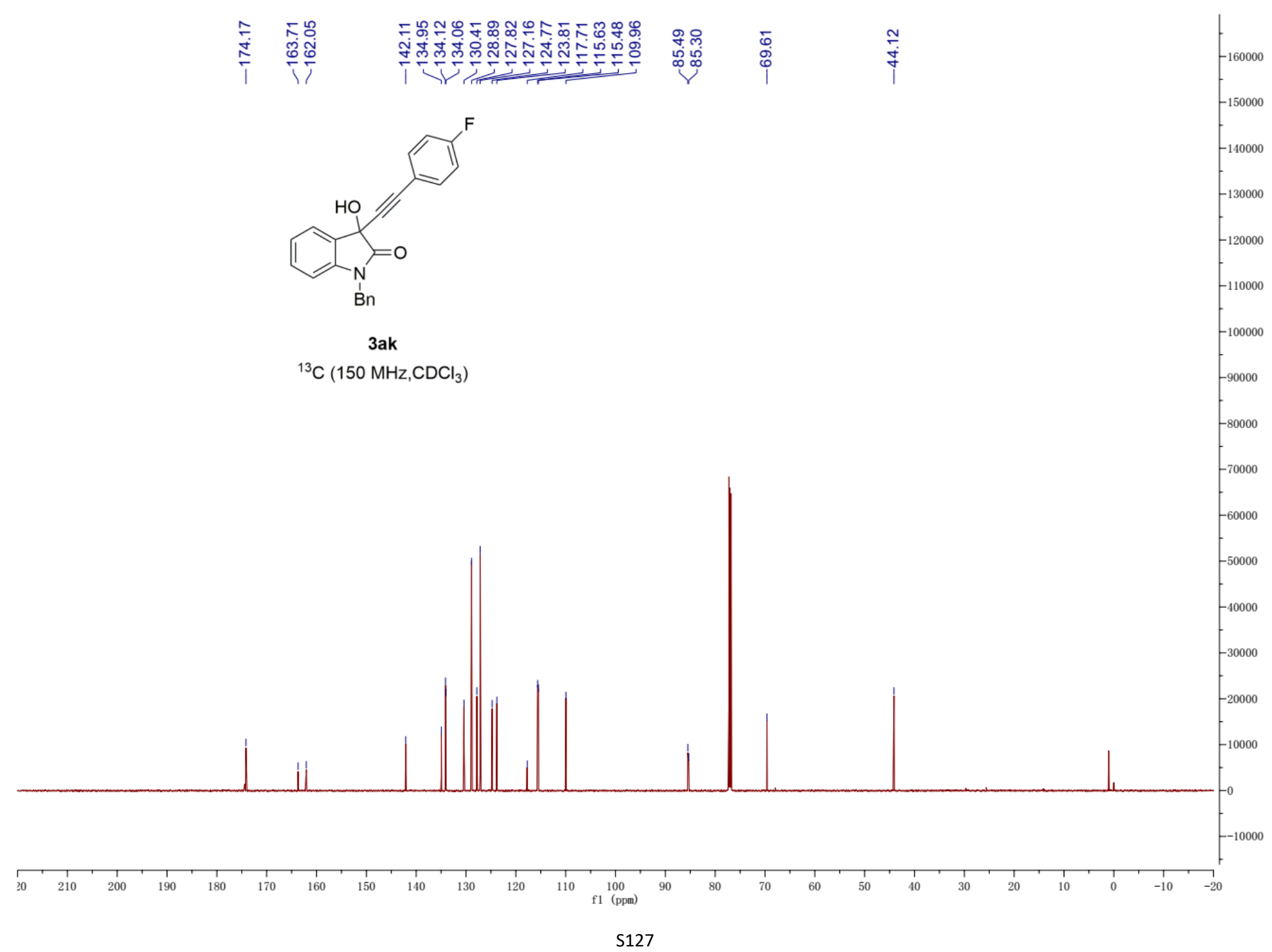




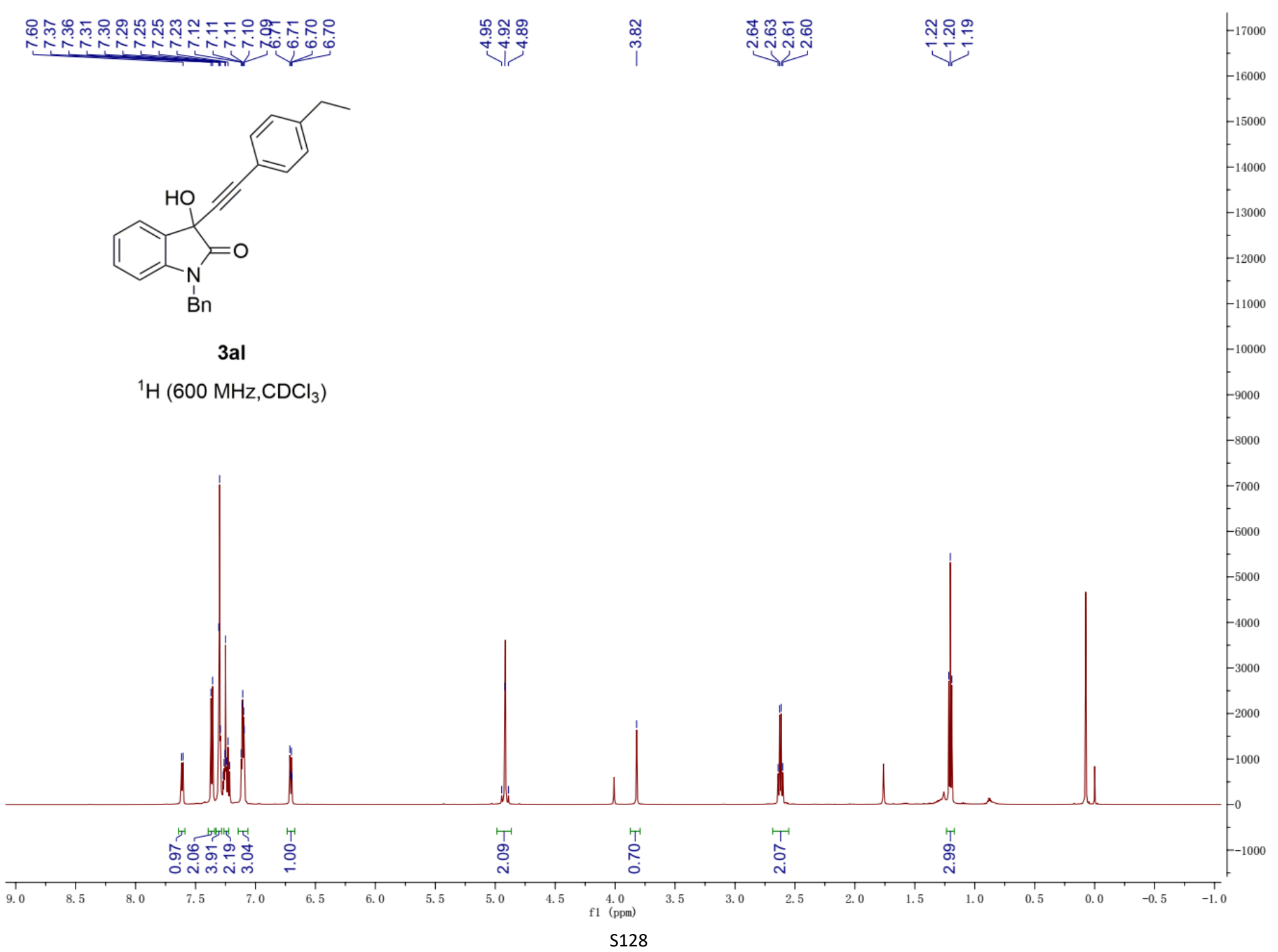




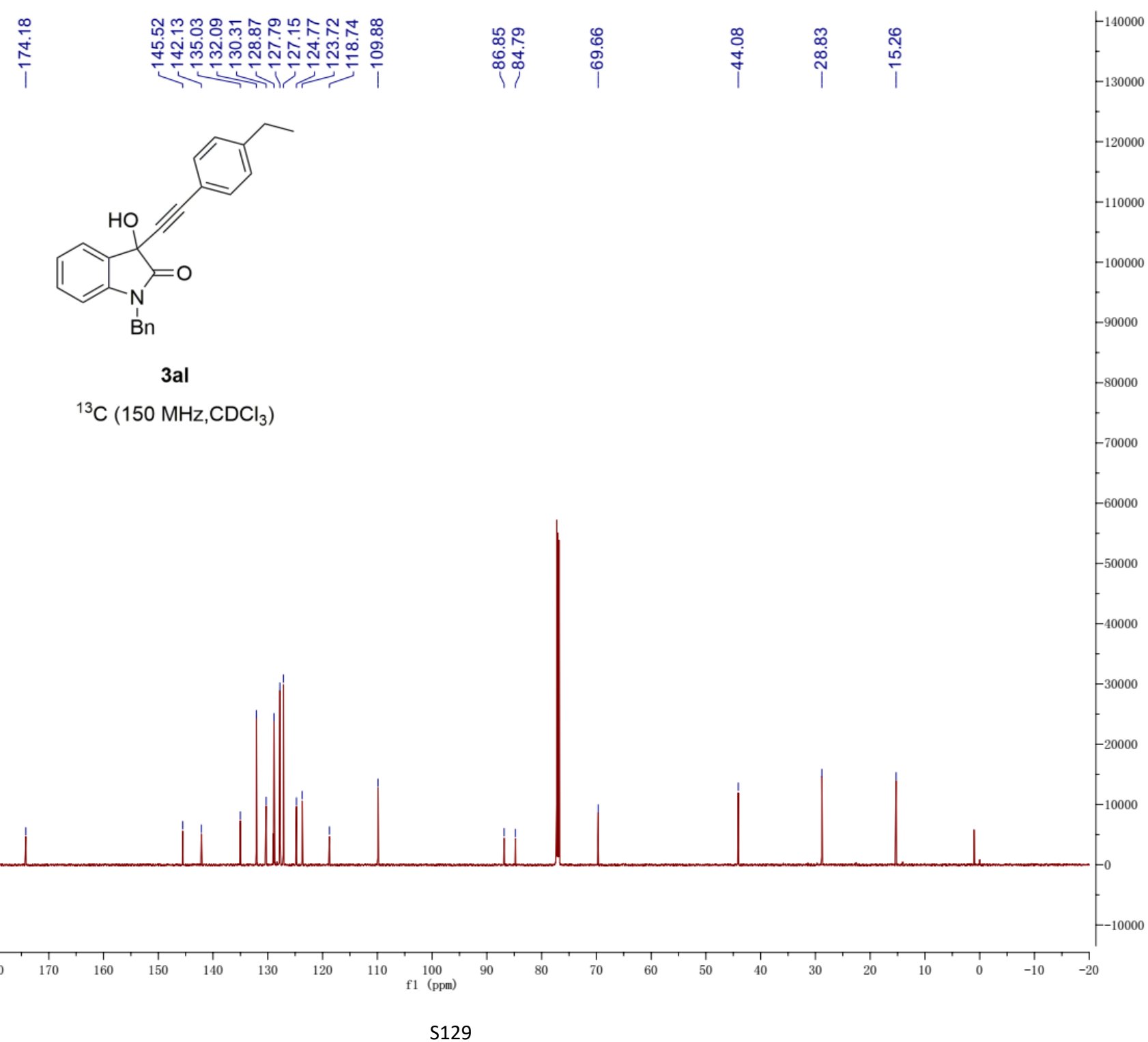




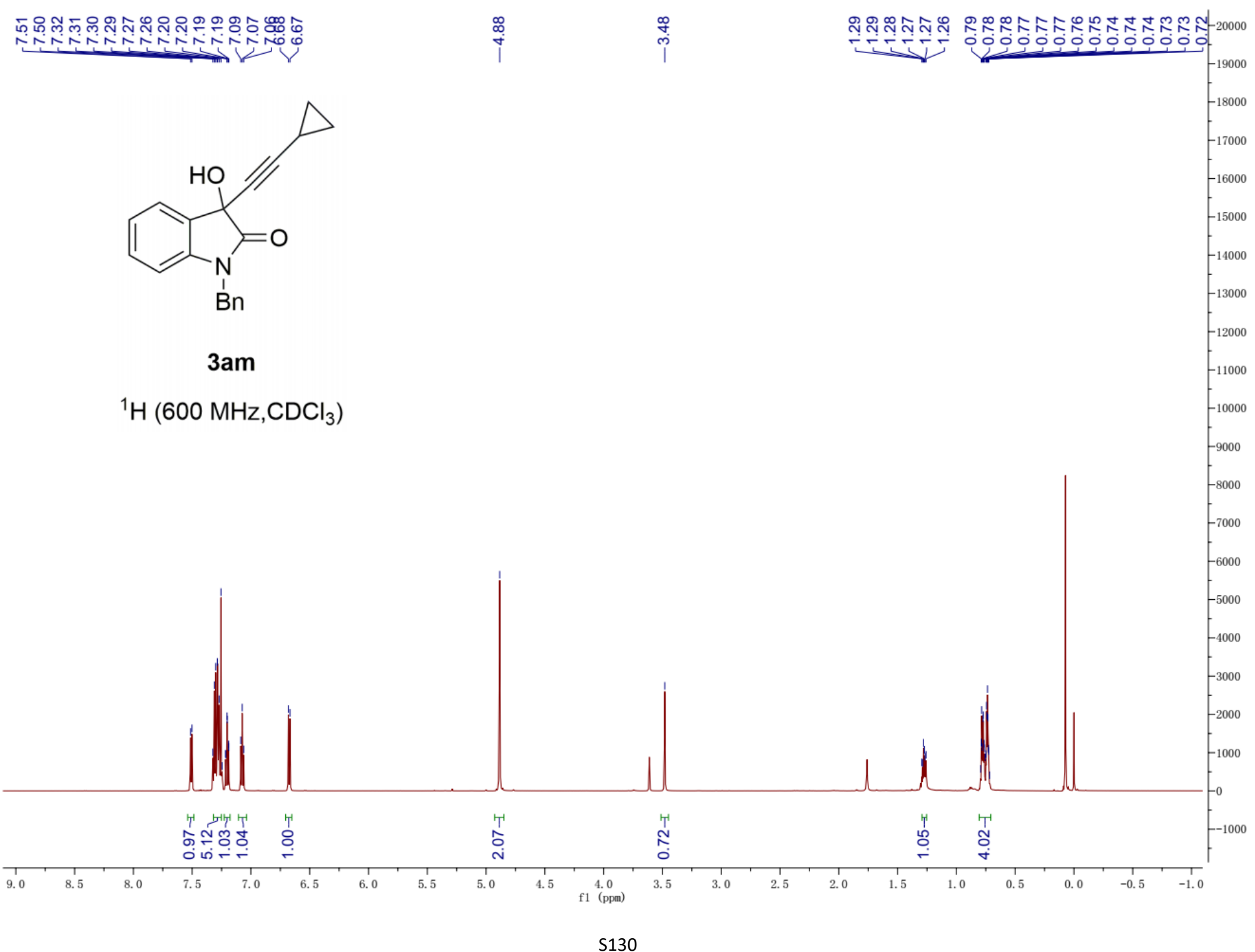




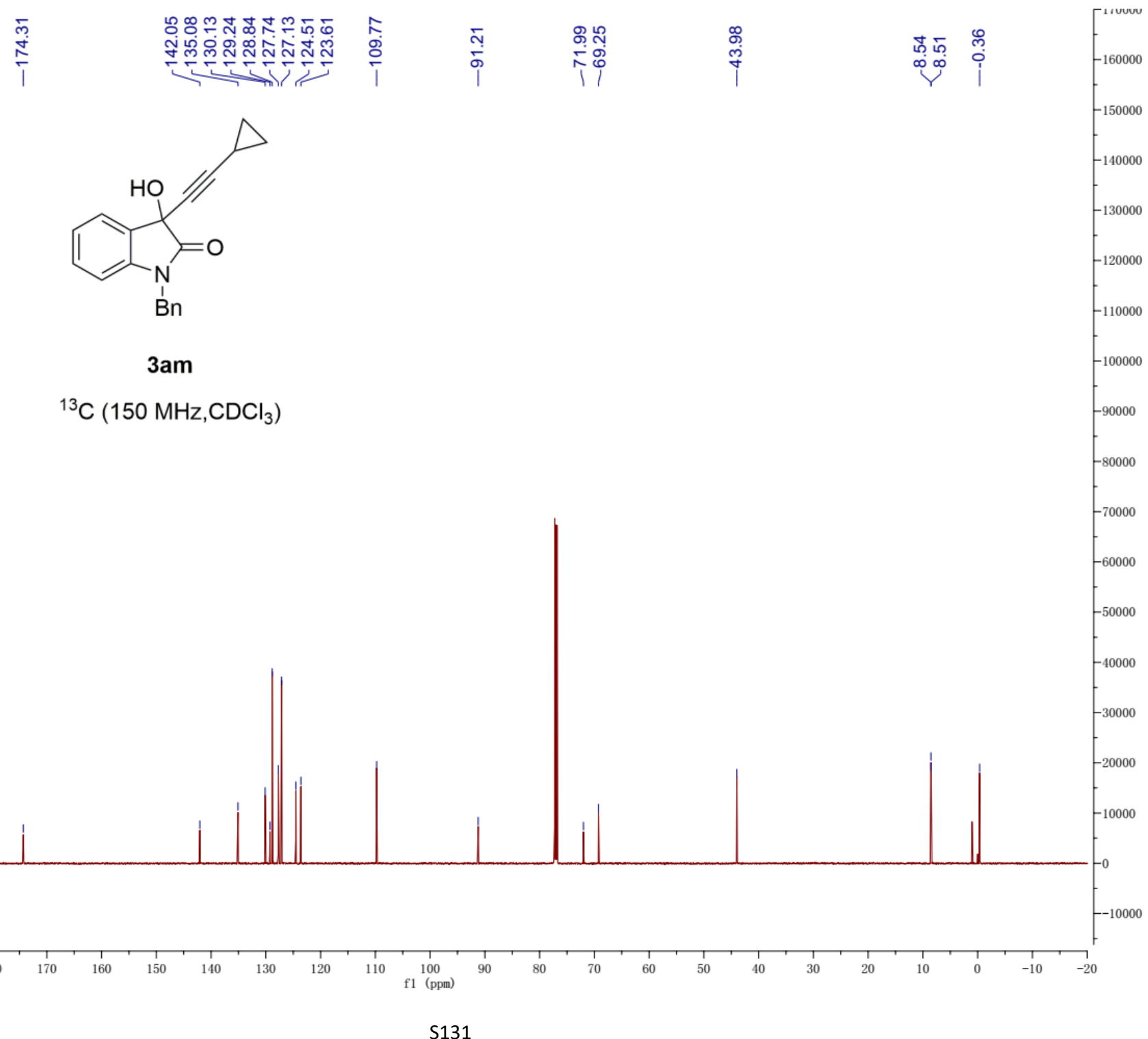

